\title{
NDTP Mediated Direct Rapid Amide and Peptide Synthesis without Epimerization
}

Yiping Li, Jingyue Li, Guangjun Bao, Changjun Yu, Yuyang Liu, Zeyuan He, Peng Wang, Wen Ma, Junqiu Xie, Wangsheng Sun*, Rui Wang*

Correspondence to: sunws@1zu.edu.cn; wangrui@1zu.edu.cn

\section{Index}

$\begin{array}{ll}\text { 1. Materials and Methods } & \text { S2 }\end{array}$

2. Procedure for the syntheses of 4, 6-dithiocyano-5-nitropyrimidine (NDTP) S4

3. Coupling Reagents Screening S5

4. Reaction Condition Optimization $\quad$ S6

5. Classic Experimental Procedure $\quad$ S8

6. Characterization of Products $\quad$ S9

7. Scale-up of the Reaction $\quad$ S33

8. Comparative Study of NDTP with Other Coupling Reagents $\quad$ S39

9. Mechanical Study S40

10. NDTP Recycling Procedure S41

11. Procedures for the Synthesis of Peptides on Resin (SPPS) S43

$\begin{array}{ll}\text { 12. Copies of NMR Spectra } & \text { S50 }\end{array}$ 


\section{Materials and Methods}

\subsection{Reagents and Solvents}

Unless otherwise noted, all reactions were carried out in conventional glassware, reagents were purchased from commercial suppliers and used directly without further purification. Technical grade solvents were purchased from commercial suppliers and used without further purification. Solid phase peptide synthesis was performed on rink amide-MBHA resin (100-200 mesh, $0.43 \mathrm{mmol} / \mathrm{g}$, crosslinked polystyrene) in a $20 \mathrm{~mL}$ filtration tube.

\subsection{Analysis and Characterization}

${ }^{1} \mathbf{H}$ and ${ }^{13} \mathbf{C}$ NMR spectra were recorded on a Bruker instrument $(300 \mathrm{MHz}$ and $75 \mathrm{MHz}$, respectively) and internally referenced to tetramethylsilane signal or residual protio solvent signals. Data for ${ }^{1} \mathbf{H}$ NMR were recorded as follows: chemical shift $(\delta, \mathrm{ppm})$, multiplicity ( $\mathrm{s}=$ singlet, $\mathrm{d}=$ doublet, $\mathrm{t}=$ triplet, $\mathrm{m}=$ multiplet, $\mathrm{q}=$ quartet or unresolved, coupling constant $(\mathrm{s})$ in $\mathrm{Hz}$, integration). Data for ${ }^{13} \mathbf{C}$ NMR were reported in terms of chemical shift $(\delta, \mathrm{ppm})$. High resolution mass spectra (HRMS) were obtained by the ESI or APCI ionization sources. Semi-preparative HPLC was carried out on a Waters 2996 using a Dubhe C18 $(10 \mu \mathrm{m}, 20 \times 250 \mathrm{~mm})$ preparative column. Linear gradients using $\mathrm{A}$ : $\mathrm{MeCN}\left(0.1 \% \mathrm{CF}_{3} \mathrm{COOH}\right)$ and $\mathrm{B}: \mathrm{H}_{2} \mathrm{O}\left(0.1 \% \mathrm{CF}_{3} \mathrm{COOH}\right)$ were run over varying periods of time. Purity analyzing was carried out on Waters e2695/2998 using XBridge ${ }^{\circledR}$ Peptide BEH C18 $(10 \mu \mathrm{m}, 4.6 \mathrm{~mm} \times 250 \mathrm{~mm})$ Column and Agilent 6100 LC/MS using Agilent 5 HC-C18 (2) $(250 \times 4.6 \mathrm{~mm})$ column. The determination of epimerization was carried out using chiral HPLC with Daicel Chiracel column on Waters with a 996 UV-detector.

\subsection{Method for semi-preparative HPLC}

Semi-preparative HPLC was carried out on a Waters 2996 using a Dubhe C18 $(10 \mu \mathrm{m}, 20 \times 250 \mathrm{~mm})$ preparative column, with the mobile phase as follows:

\begin{tabular}{cccc}
\hline Time $(\min )$ & A $(\%)$ & B (\%) & Flow $(\mathrm{mL} / \mathrm{min})$ \\
\hline 0.0 & 10.0 & 90.0 & 8.0 \\
5.0 & 10.0 & 90.0 & 8.0 \\
40.0 & 40.0 & 60.0 & 8.0 \\
50.0 & 50.0 & 50.0 & 8.0 \\
60.0 & 100.0 & 0.0 & 8.0
\end{tabular}

Linear gradients using $\mathrm{A}$ : $\mathrm{MeCN}\left(0.1 \% \mathrm{CF}_{3} \mathrm{COOH}\right)$ and $\mathrm{B}: \mathrm{H}_{2} \mathrm{O}\left(0.1 \% \mathrm{CF}_{3} \mathrm{COOH}\right)$.

\subsection{Method for analyzing the purities}


Purity analyzing was carried out on Waters e2695/2998 using XBridge ${ }^{\circledR}$ Peptide BEH C18 (10 $\mu \mathrm{m}, 4.6 \mathrm{~mm} \times 250 \mathrm{~mm})$ Column and Agilent $6100 \mathrm{LC} / \mathrm{MS}$ using Agilent 5 HC-C18 (2) (250 x 4.6 $\mathrm{mm})$ column.

\begin{tabular}{cccc}
\hline Time $(\mathrm{min})$ & $\mathrm{A}(\%)$ & $\mathrm{C}(\%)$ & Flow $(\mathrm{mL} / \mathrm{min})$ \\
\hline 0.0 & 10.0 & 90.0 & 1.0 \\
30.0 & 90.0 & 10.0 & 1.0 \\
35.0 & 100.0 & 0.0 & 1.0 \\
40.0 & 10.0 & 90.0 & 1.0 \\
\hline
\end{tabular}

Linear gradients of HPLC using A: $\mathrm{MeCN}\left(0.1 \% \mathrm{CF}_{3} \mathrm{COOH}\right)$ and $\mathrm{B}: \mathrm{H}_{2} \mathrm{O}\left(0.1 \% \mathrm{CF}_{3} \mathrm{COOH}\right)$.

\begin{tabular}{cccc}
\hline Time $(\mathrm{min})$ & $\mathrm{A}(\%)$ & $\mathrm{B}(\%)$ & Flow $(\mathrm{mL} / \mathrm{min})$ \\
\hline 0.0 & 80.0 & 20.0 & 0.5 \\
6.0 & 0.0 & 100.0 & 1.0 \\
12.0 & 0.0 & 100.0 & 1.0 \\
15.0 & 80.0 & 100.0 & 1.0 \\
\hline
\end{tabular}

Linear gradients of LC/MS using $\mathrm{A}: \mathrm{H}_{2} \mathrm{O}+0.1 \%$ Formic acid and $\mathrm{B}$ : MeCN. 


\section{Procedure for the syntheses of 4, 6-dithiocyano-5-nitropyrimidine (NDTP)}

4,6-dichloro-5-nitropyrimidine (1.94 g, $10 \mathrm{mmol})$ was dissolved in $\mathrm{AcOH}(20 \mathrm{~mL})$. Potassium thiocyanate $(5.82 \mathrm{~g}, 60 \mathrm{mmol})$ was added in batches and stirred at room temperature. The reaction mixture was diluted with ice water when a large amount of yellow solid precipitated. The precipitate was filtered off, washed with water, and dried. Then, the yellow solid was washed with hexane (or EtOAc: hexane =1:20). The filtrate was concentrated under vacuum which afforded the unreacted 4, 6-dichloro-5-nitropyrimidine. The solid was dissolved with DCM and filtered by silica gel. The filtrate was concentrated under vacuum to afford the product NDTP as pale yellow solid (1.54 g, 64\%).

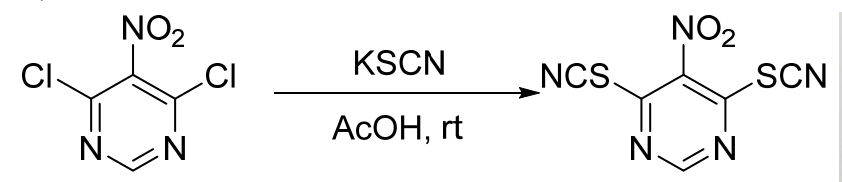

NDTP<smiles>N#[Sb]c1ncnc(S[Na])c1[N+](=O)[O-]</smiles>

NDTP

${ }^{1} \mathbf{H}$ NMR $\left(300 \mathrm{MHz}, \mathrm{CDCl}_{3}\right) \delta 9.23(\mathrm{~s}, 1 \mathrm{H}) ;{ }^{13} \mathbf{C}$ NMR $\left(75 \mathrm{MHz}, \mathrm{CDCl}_{3}\right) \delta 163.4,158.0,105.0$; HRMS (FTMS + p ESI) m/z: $[\mathrm{M}+\mathrm{H}]^{+}$calcd for $\mathrm{C}_{6} \mathrm{H}_{2} \mathrm{~N}_{5} \mathrm{O}_{2} \mathrm{~S}_{2} 239.9644$, found: 239.9648 . 


\section{Coupling Reagents Screening}

To a solution of Fmoc-Gly-OH (0.15 mmol), L-Alanine methyl ester hydrochloride $(0.10 \mathrm{mmol})$ and DIPEA $(0.4 \mathrm{mmol})$ in $\mathrm{MeCN}(0.5 \mathrm{~mL})$ was added the solution of condensation reagent $\mathbf{S 1}$ $(0.15 \mathrm{mmol})$ in $\mathrm{MeCN}(0.5 \mathrm{~mL})$ and stirred for 1 minute. Then the solvent was evaporated under reduced pressure, and the residue was purified by column chromatography (EtOAc:hexane $=1: 1$ ).

Table S1. Amide coupling reagent screening.

(n)




\section{Reaction Condition Optimization}

We optimized the reaction conditions, such as solvents, base and ratios between substrates and NDTP (Table S2, entries 1-12). The results exhibited that various frequently-used solvents, such as $\mathrm{MeCN}$, DCM, DMF, even EtOAc, could tolerate the methodology well, giving the dipeptide 3aa in higher than 90\% yield (Table S2, entries 1-4), while THF and Acetone could only provide moderate yield (Table S2, entries 5-6). Other bases, such as TEA or NMM could enable the amidation with moderate to good yield (Table S2, entries 7-8). When the amount of DIPEA was reduced to 2 or 3 eq., the yield dropped slightly to $90 \%$ or $96 \%$, respectively (Table S2, entries 910). The ratio of the substrates was then examined, it showed that the acid component should slightly excessive to the amine component to ensure the quantitative transformation (Table S2, entries 11-13). In addition, lowering NDTP to 1 eq. could slightly erode the yield of the methodology (Table S2, entry 14). As a result, we confirmed an optimal condition of stirring 1.5 eq. of acid component 1a, 1 eq. of methyl L-alaninate hydrochloride $2 \mathrm{a}$ and 4 eq. of DIPEA in 0.5 $\mathrm{mL} \mathrm{MeCN}$, followed by the addition of 1.5 eq. NDTP in $0.5 \mathrm{~mL} \mathrm{MeCN}$ at ambient temperature (Table S2, entry 13)

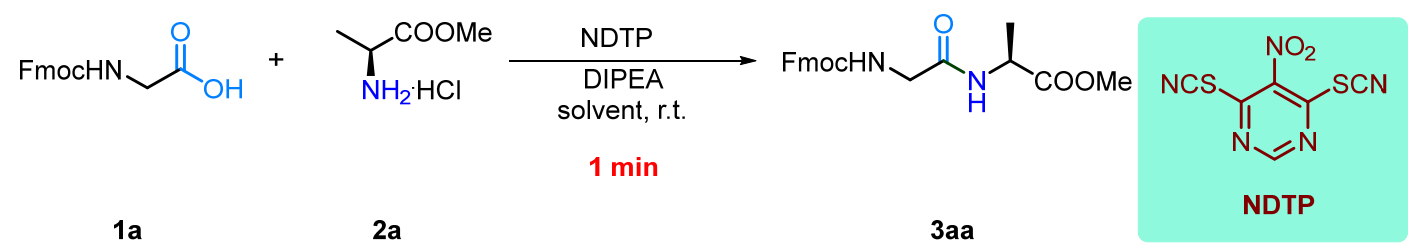

Table S2. Reaction Condition Optimization. ${ }^{[a]}$

\begin{tabular}{lllll}
\hline entry & $1 \mathrm{a} / 2 \mathrm{a}$ & base & solvent & ${\text { Yield }(\%)^{[\mathrm{b}]}}^{\mathrm{m}}$ \\
\hline 1 & 2 & DIPEA & MeCN & $>99$ \\
2 & 2 & DIPEA & DCM & 92 \\
3 & 2 & DIPEA & EtOAc & 95 \\
4 & DIPEA & DMF & 90 \\
5 & 2 & DIPEA & THF & 52 \\
6 & 2 & DIPEA & Acetone & 54
\end{tabular}




$\begin{array}{llllc}7 & 2 & \text { TEA } & \text { MeCN } & 80 \\ 8 & 2 & \text { NMM } & \text { MeCN } & 75 \\ 9^{[\mathrm{c}]} & 2 & \text { DIPEA } & \text { MeCN } & 90 \\ 10^{[\mathrm{d}]} & 2 & \text { DIPEA } & \mathrm{MeCN} & 96 \\ 11 & 1 & \text { DIPEA } & \mathrm{MeCN} & 78 \\ 12 & 2 / 3 & \text { DIPEA } & \mathrm{MeCN} & 88 \\ 13 & 1.5 & \text { DIPEA } & \mathrm{MeCN} & >99(>99)^{[\mathrm{ee}]} \\ 14^{[\mathrm{f}]} & 1.5 & \text { DIPEA } & \mathrm{MeCN} & 90\end{array}$

[a] Unless otherwise stated, the reaction was carried out in $0.1 \mathrm{mmol}$ scale as Method A. [b] Yield refers to isolated yield after flash column chromatography. [c] DIPEA was used in 2 eq. [d] DIPEA was used in 3 eq. [e] ee was given in parenthesis after chiral HPLC analysis. [f] 1 eq. of NDTP was used. NDTP refers to 5-nitro-4,6-dithiocyanatopyrimidine, DIPEA refers to N,NDiisopropylethylamine, TEA refers to Triethyl-amine, NMM refers to 4-Methylmorpholine, DMF refers to N,NDimethylformamide, THF refers to Tetrahydrofuran. 


\section{Classic Experimental Procedure}

Method A: To a solution of carboxylic acid $(0.15 \mathrm{mmol})$, amine hydrochloride $(0.10 \mathrm{mmol})$ and DIPEA $(0.4 \mathrm{mmol}, 4 \mathrm{eq})$ in $\mathrm{MeCN}(0.5 \mathrm{~mL})$ was added the solution of NDTP $(0.15 \mathrm{mmol})$ in $\mathrm{MeCN}(0.5 \mathrm{~mL})$ and stirred for 1 minute. Then the solvent was evaporated under reduced pressure, and the residue was purified by flash column chromatography (EtOAc/hexane $=1: 1-1: 3$ or $\left.\mathrm{MeOH} / \mathrm{CH}_{2} \mathrm{Cl}_{2}=1: 10\right)$.<smiles>[R]C(N)C(=O)OC(=O)C([R])NC(=O)C([R])NC(=O)OC</smiles>

Method B: To a solution of carboxylic acid $(0.15 \mathrm{mmol})$, amine $(0.10 \mathrm{mmol})$ and DIPEA (0.3 mmol, 3 eq $)$ in $\mathrm{MeCN}(0.5 \mathrm{~mL})$ was added the solution of NDTP $(0.15 \mathrm{mmol})$ in $\mathrm{MeCN}(0.5 \mathrm{~mL})$ and stirred for 1 minute. Then the solvent was evaporated under reduced pressure, and the residue was purified by flash column chromatography $\left(\right.$ EtOAc/hexane $=1: 1-1: 3$ or $\left.\mathrm{MeOH} / \mathrm{CH}_{2} \mathrm{Cl}_{2}=1: 10\right)$.

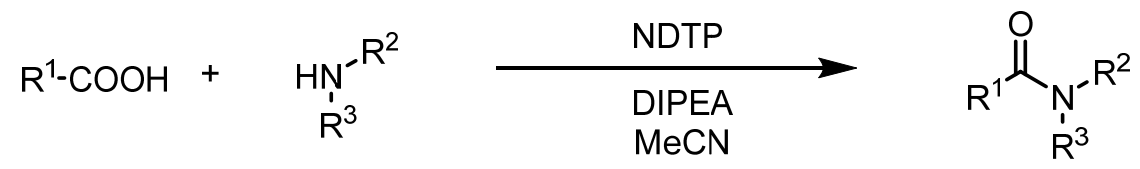

Method C: To a solution of carboxylic acid $(0.15 \mathrm{mmol})$, amine $(0.10 \mathrm{mmol})$ and DABCO $(0.6$ mmol, 6 eq) in MeCN $(0.5 \mathrm{~mL})$ was added the solution of NDTP $(0.15 \mathrm{mmol})$ in $\mathrm{MeCN}(0.5 \mathrm{~mL})$ and stirred for 1 minute. Then the solvent was evaporated under reduced pressure, and the residue was purified by column chromatography (EtOAc/hexane $=1: 1-1: 3$ or $\mathrm{MeOH} / \mathrm{CH}_{2} \mathrm{Cl}_{2}=1: 10$ ).

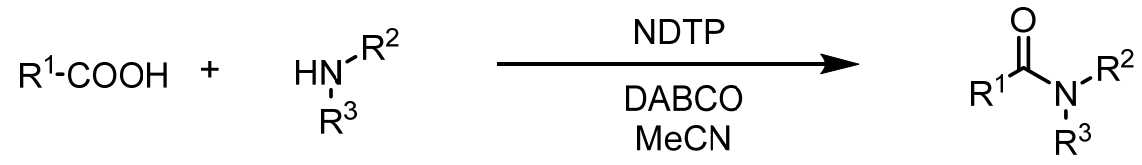




\section{Characterization of Products}

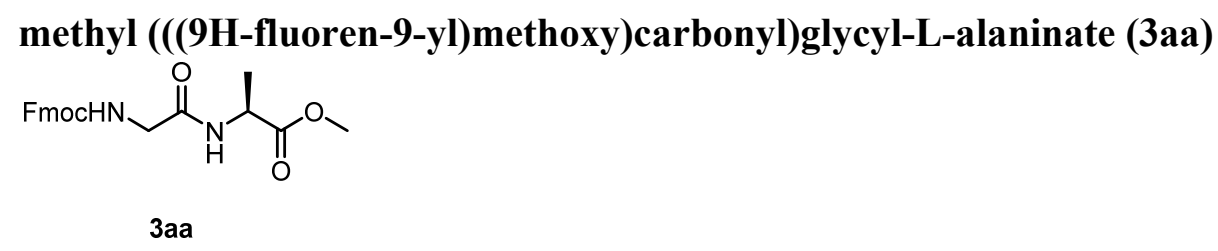

Method A, Off-white solid; $38.1 \mathrm{mg},>99 \%$ yield. ${ }^{1} \mathbf{H}$ NMR $\left(300 \mathrm{MHz}, \mathrm{CDCl}_{3}\right) \delta 7.69$ (d, $J=7.5$ $\mathrm{Hz}, 2 \mathrm{H}), 7.55$ (d, $J=7.5 \mathrm{~Hz}, 2 \mathrm{H}), 7.32$ (t, $J=7.3 \mathrm{~Hz}, 3 \mathrm{H}), 7.23$ (t, $J=7.4 \mathrm{~Hz}, 2 \mathrm{H}), 6.24(\mathrm{~s}, 1 \mathrm{H})$, $4.57(\mathrm{t}, J=7.3 \mathrm{~Hz}, 1 \mathrm{H}), 4.33(\mathrm{~d}, J=7.2 \mathrm{~Hz}, 2 \mathrm{H}), 4.16(\mathrm{~d}, J=7.1 \mathrm{~Hz}, 1 \mathrm{H}), 3.94(\mathrm{~d}, J=5.6 \mathrm{~Hz}$, 2H), $3.64(\mathrm{~s}, 3 \mathrm{H}), 1.35$ (d, $J=7.2 \mathrm{~Hz}, 3 \mathrm{H}) ;{ }^{13} \mathbf{C ~ N M R}\left(75 \mathrm{MHz}, \mathrm{CDCl}_{3}\right) \delta 173.1,168.9,156.5$, 143.4, 140.9, 127.4, 126.7, 124.8, 119.7, 66.9, 52.1, 47.8, 46.7, 43.9, 17.6; HRMS (ESI) m/z: $[\mathrm{M}+\mathrm{Na}]^{+}$calcd for $\mathrm{C}_{21} \mathrm{H}_{22} \mathrm{~N}_{2} \mathrm{O}_{5} \mathrm{Na}$ 405.1421, found: 405.1439; HPLC (Chiralpak ${ }^{\circledR} \mathrm{IC}$ column, ethanol $/ \mathrm{n}$-hexane $=2 / 8$, flow rate $=1.0 \mathrm{~mL} / \mathrm{min}, 1=254 \mathrm{~nm}$ ) $\mathrm{t}^{\mathrm{R}}=13.1 \mathrm{~min}$ (major), $17.2 \mathrm{~min}$ (minor).

methyl (((9H-fluoren-9-yl)methoxy)carbonyl)glycyl-L-leucinate (3ab)<smiles>COC(=O)C(CC(C)C)NC(=O)CNC(F)F</smiles>

$3 a b$

Method A, Off-white solid; $38.6 \mathrm{mg}, 91 \%$ yield. ${ }^{1} \mathbf{H}$ NMR $\left(300 \mathrm{MHz}, \mathrm{CDCl}_{3}\right) \delta 7.74(\mathrm{~d}, J=7.5$ $\mathrm{Hz}, 2 \mathrm{H}), 7.58(\mathrm{~d}, J=7.5 \mathrm{~Hz}, 2 \mathrm{H}), 7.38$ (t, $J=7.4 \mathrm{~Hz}, 2 \mathrm{H}), 7.28$ (td, $J=7.6,1.4 \mathrm{~Hz}, 2 \mathrm{H}), 6.78$ (d, $J=8.3 \mathrm{~Hz}, 1 \mathrm{H}), 5.80(\mathrm{t}, J=5.6 \mathrm{~Hz}, 1 \mathrm{H}), 4.64(\mathrm{~m}, 1 \mathrm{H}), 4.38(\mathrm{~d}, J=7.0 \mathrm{~Hz}, 2 \mathrm{H}), 4.20(\mathrm{t}, J=7.1$ $\mathrm{Hz}, 1 \mathrm{H}), 3.95(\mathrm{t}, J=4.8 \mathrm{~Hz}, 2 \mathrm{H}), 3.70(\mathrm{~s}, 3 \mathrm{H}), 1.71-1.45(\mathrm{~m}, 3 \mathrm{H}), 0.98-0.81(\mathrm{~m}, 6 \mathrm{H}) ;{ }^{13} \mathbf{C ~ N M R}$ $\left(75 \mathrm{MHz}, \mathrm{CDCl}_{3}\right) \delta 173.3,168.9,156.6,143.6,141.2,127.7,127.0,125.0,119.9,67.2,52.3,50.7$, 46.9, 44.2, 41.3, 24.7, 22.7, 21.8; HRMS (ESI) m/z: $[\mathrm{M}+\mathrm{Na}]^{+}$calcd for $\mathrm{C}_{24} \mathrm{H}_{28} \mathrm{~N}_{2} \mathrm{O}_{5} \mathrm{Na} 447.1890$, found: 447.1908; HPLC (Chiralpak ${ }^{\circledR} \mathrm{IC}$ column, ethanol $/ \mathrm{n}$-hexane $=2 / 8$, flow rate $=1.0 \mathrm{~mL} / \mathrm{min}$, $1=254 \mathrm{~nm}) \mathrm{t}^{\mathrm{R}}=8.7 \min$ (major), $11.0 \min$ (minor).

methyl (((9H-fluoren-9-yl)methoxy)carbonyl)glycylglycinate (3ac)<smiles>COC(=O)CNCC(=O)NCC(=O)OC</smiles>

3ac 
Method A, Off-white solid; $31.7 \mathrm{mg}, 86 \%$ yield. ${ }^{1} \mathbf{H}$ NMR $\left(300 \mathrm{MHz}, \mathrm{CDCl}_{3}\right) \delta 7.75$ (d, $J=7.6$ $\mathrm{Hz}, 2 \mathrm{H}), 7.58$ (d, $J=7.2 \mathrm{~Hz}, 2 \mathrm{H}), 7.39$ (t, $J=7.4 \mathrm{~Hz}, 2 \mathrm{H}), 7.34-7.25(\mathrm{~m}, 2 \mathrm{H}), 6.67(\mathrm{~s}, 1 \mathrm{H}), 5.64$ $(\mathrm{t}, J=5.8 \mathrm{~Hz}, 1 \mathrm{H}), 4.43(\mathrm{~d}, J=6.9 \mathrm{~Hz}, 2 \mathrm{H}), 4.21(\mathrm{t}, J=6.9 \mathrm{~Hz}, 1 \mathrm{H}), 4.04(\mathrm{~d}, J=5.3 \mathrm{~Hz}, 2 \mathrm{H}), 3.93$ $(\mathrm{d}, J=5.7 \mathrm{~Hz}, 2 \mathrm{H}), 3.73(\mathrm{~s}, 3 \mathrm{H}) ;{ }^{13} \mathbf{C} \mathbf{~ N M R}\left(75 \mathrm{MHz}, \mathrm{CDCl}_{3}\right) \delta 170.1,169.3,156.7,143.7,141.3$, 127.8, 127.1, 125.0, 120.0, 67.2, 52.5, 47.1, 44.4, 41.1; HRMS (ESI) m/z: [M+Na $]^{+}$calcd for $\mathrm{C}_{20} \mathrm{H}_{20} \mathrm{~N}_{2} \mathrm{O} 5 \mathrm{Na} 391.1264$, found: 391.1281 .

methyl (((9H-fluoren-9-yl)methoxy)carbonyl)glycyl-L-phenylalaninate (3ad)<smiles>COC(=O)C(Cc1ccccc1)NC(=O)CNC(F)F</smiles>

3ad

Method A, Off-white solid; $41.7 \mathrm{mg}, 91 \%$ yield. ${ }^{1} \mathbf{H}$ NMR $\left(300 \mathrm{MHz}, \mathrm{CDCl}_{3}\right) \delta 7.74(\mathrm{~d}, J=7.5$ $\mathrm{Hz}, 2 \mathrm{H}), 7.56(\mathrm{~d}, J=7.5 \mathrm{~Hz}, 2 \mathrm{H}), 7.38$ (t, $J=7.4 \mathrm{~Hz}, 2 \mathrm{H}), 7.28(\mathrm{~m}, 2 \mathrm{H}), 7.24-7.11(\mathrm{~m}, 3 \mathrm{H}), 7.06$ $(\mathrm{d}, J=6.7 \mathrm{~Hz}, 2 \mathrm{H}), 6.76(\mathrm{~d}, J=8.0 \mathrm{~Hz}, 1 \mathrm{H}), 5.70(\mathrm{t}, J=5.6 \mathrm{~Hz}, 1 \mathrm{H}), 4.92-4.81(\mathrm{~m}, 1 \mathrm{H}), 4.35(\mathrm{~d}$, $J=7.4 \mathrm{~Hz}, 2 \mathrm{H}), 4.18(\mathrm{t}, J=7.1 \mathrm{~Hz}, 1 \mathrm{H}), 3.85$ (dd, $J=5.7,3.0 \mathrm{~Hz}, 2 \mathrm{H}), 3.67$ (s, 3H), 3.07 (m, 2H); ${ }^{13}$ C NMR $\left(75 \mathrm{MHz}, \mathrm{CDCl}_{3}\right) \delta 171.7,168.6,156.4,143.6,141.1,135.5,129.1,128.5,127.6,127.1$, 127.0, 125.0, 119.9, 67.2, 53.1, 52.3, 46.9, 44.2, 37.7; HRMS (ESI) m/z: $[\mathrm{M}+\mathrm{Na}]^{+}$calcd for $\mathrm{C}_{27} \mathrm{H}_{26} \mathrm{~N}_{2} \mathrm{O}_{5} \mathrm{Na}^{+} 481.1734$, found: 481.1750; HPLC (Chiralpak ${ }^{\circledR} \mathrm{IC}$ column, ethanol/n-hexane = $2 / 8$, flow rate $=1.0 \mathrm{~mL} / \mathrm{min}, 1=254 \mathrm{~nm}$ ) $\mathrm{t}^{\mathrm{R}}=12.9 \min$ (major), $19.7 \mathrm{~min}$ (minor).

\section{methyl (((9H-fluoren-9-yl)methoxy)carbonyl)glycyl-L-tryptophanate (3ae)}<smiles>COC(=O)[C@H](Cc1c[nH]c2ccccc12)NC(=O)CNC(F)F</smiles>

3ae

Method A, Off-white solid; $45.9 \mathrm{mg}, 92 \%$ yield. ${ }^{1} \mathbf{H}$ NMR (300 MHz, $\left.\mathrm{CDCl}_{3}\right) \delta 8.32(\mathrm{~s}, 1 \mathrm{H}), 7.73$ $(\mathrm{d}, J=7.5 \mathrm{~Hz}, 2 \mathrm{H}), 7.52$ (t, $J=6.3 \mathrm{~Hz}, 2 \mathrm{H}), 7.44$ (d, $J=7.6 \mathrm{~Hz}, 1 \mathrm{H}), 7.36$ (t, $J=7.5 \mathrm{~Hz}, 2 \mathrm{H}), 7.30$ $-7.17(\mathrm{~m}, 3 \mathrm{H}), 7.06(\mathrm{~m}, J=13.3,7.1,3.6 \mathrm{~Hz}, 2 \mathrm{H}), 6.88-6.73(\mathrm{~m}, 2 \mathrm{H}), 5.59(\mathrm{t}, J=5.7 \mathrm{~Hz}, 1 \mathrm{H})$, $4.93-4.83(\mathrm{~m}, 1 \mathrm{H}), 4.28(\mathrm{~d}, J=6.7 \mathrm{~Hz}, 2 \mathrm{H}), 4.12(\mathrm{t}, J=7.1 \mathrm{~Hz}, 1 \mathrm{H}), 3.72(\mathrm{~d}, J=5.7 \mathrm{~Hz}, 2 \mathrm{H})$, 
$3.60(\mathrm{~s}, 3 \mathrm{H}), 3.25(\mathrm{~d}, J=5.5 \mathrm{~Hz}, 2 \mathrm{H}) ;{ }^{13} \mathbf{C}$ NMR $\left(75 \mathrm{MHz}, \mathrm{CDCl}_{3}\right) \delta 172.2,168.9,156.5,143.6$, $141.1,135.9,127.7,127.3,127.0,125.0,123.1,122.0,119.9,119.5,118.2,111.3,109.2$, 67.1, 52.7, 52.4, 46.9, 44.1, 27.3; HRMS (ESI) m/z: $[\mathrm{M}+\mathrm{Na}]^{+}$calcd for $\mathrm{C}_{29} \mathrm{H}_{27} \mathrm{~N}_{3} \mathrm{O}_{5} \mathrm{Na} 520.1843$, found: 520.1861; HPLC (Chiralpak ${ }^{\circledR}$ IC column, ethanol $/ \mathrm{n}$-hexane $=2 / 8$, flow rate $=1.0 \mathrm{~mL} / \mathrm{min}, 1=254$ $\mathrm{nm}) \mathrm{t}^{\mathrm{R}}=13.9 \min$ (major), $19.4 \min$ (minor).

\section{methyl (((9H-fluoren-9-yl)methoxy)carbonyl)glycyl-L-tyrosinate (3af)}<smiles>COC(=O)C(Cc1ccc(O)cc1)NC(=O)CNC(F)F</smiles>

3af

Method A, Off-white solid; $42.5 \mathrm{mg}, 90 \%$ yield. ${ }^{1} \mathbf{H}$ NMR $\left(300 \mathrm{MHz}\right.$, Methanol- $\left.d_{4}\right) \delta 8.06(\mathrm{~d}, \mathrm{~J}=$ $7.8 \mathrm{~Hz}, 1 \mathrm{H}), 7.77$ (d, J = 7.4 Hz, 2H), 7.65 (d, J = 7.4 Hz, 2H), $7.46-7.34$ (m, 2H), 7.29 (t, J = 7.4 $\mathrm{Hz}, 2 \mathrm{H}), 6.98(\mathrm{~d}, \mathrm{~J}=8.2 \mathrm{~Hz}, 2 \mathrm{H}), 6.81-6.53(\mathrm{~m}, 2 \mathrm{H}), 4.89(\mathrm{~s}, 3 \mathrm{H}), 4.63(\mathrm{td}, \mathrm{J}=7.8,5.8 \mathrm{~Hz}, 1 \mathrm{H})$, $4.33(\mathrm{~d}, \mathrm{~J}=7.5 \mathrm{~Hz}, 2 \mathrm{H}), 4.20(\mathrm{t}, \mathrm{J}=7.0 \mathrm{~Hz}, 1 \mathrm{H}), 3.76(\mathrm{~d}, \mathrm{~J}=3.4 \mathrm{~Hz}, 2 \mathrm{H}), 3.66(\mathrm{~s}, 3 \mathrm{H}), 3.31$ (p, J $=1.7 \mathrm{~Hz}, 1 \mathrm{H}), 3.02(\mathrm{dd}, \mathrm{J}=13.9,5.8 \mathrm{~Hz}, 1 \mathrm{H}), 2.90(\mathrm{dd}, \mathrm{J}=13.9,7.7 \mathrm{~Hz}, 1 \mathrm{H}) ;{ }^{13} \mathbf{C} \mathbf{N M R}(75 \mathrm{MHz}$, Methanol- $\left.d_{4}\right) \delta 172.0,170.6,157.6,156.1,143.8,141.2$, 129.9, 127.4, 127.0, 126.8, 124.8, 119.5, 114.9, 66.8, 54.1, 51.3, 46.9, 43.2, 36.3; HRMS (ESI) m/z: $[\mathrm{M}+\mathrm{Na}]^{+}$calcd for $\mathrm{C}_{27} \mathrm{H}_{26} \mathrm{~N}_{2} \mathrm{O}_{6} \mathrm{Na}$ 497.1683, found: 497.1694; HPLC (Chiralpak ${ }^{\circledR} \mathrm{IC}$ column, ethanol/n-hexane $=2 / 8$, flow rate $=$ $1.0 \mathrm{~mL} / \min , 1=254 \mathrm{~nm}) \mathrm{t}^{\mathrm{R}}=10.8 \min$ (major), $15.8 \min$ (minor).

methyl (((9H-fluoren-9-yl)methoxy)carbonyl)glycyl-L-serinate (3ag)<smiles>COC(=O)C(CO)NC(=O)CNC=C(F)F</smiles>

$3 a g$

Method A, Off-white solid; $34.9 \mathrm{mg}, 88 \%$ yield. ${ }^{1} \mathbf{H}$ NMR $\left(300 \mathrm{MHz}, \mathrm{CDCl}_{3}\right) \delta 7.72(\mathrm{~d}, J=7.5$ $\mathrm{Hz}, 2 \mathrm{H}), 7.55$ (d, $J=7.5 \mathrm{~Hz}, 2 \mathrm{H}), 7.35$ (q, $J=6.8,6.1 \mathrm{~Hz}, 3 \mathrm{H}), 7.26(\mathrm{t}, J=7.2 \mathrm{~Hz}, 2 \mathrm{H}), 5.96(\mathrm{t}, J$ $=5.6 \mathrm{~Hz}, 1 \mathrm{H}), 4.64(\mathrm{~m}, 1 \mathrm{H}), 4.35(\mathrm{~d}, J=7.1 \mathrm{~Hz}, 2 \mathrm{H}), 4.17(\mathrm{t}, J=7.0 \mathrm{~Hz}, 1 \mathrm{H}), 3.93(\mathrm{t}, J=6.1 \mathrm{~Hz}$, 4H), $3.70(\mathrm{~s}, 4 \mathrm{H}) ;{ }^{13} \mathbf{C}$ NMR (75 MHz, $\left.\mathrm{CDCl}_{3}\right) \delta$ 170.9, 169.6, 156.9, 143.6, 143.6, 141.2, 127.7, 127.0, 125.0, 119.9, 67.3, 62.5, 54.7, 52.7, 46.9, 44.1; HRMS (ESI) m/z: $[\mathrm{M}+\mathrm{Na}]^{+}$calcd for 
$\mathrm{C}_{21} \mathrm{H}_{22} \mathrm{~N}_{2} \mathrm{O}_{6} \mathrm{Na} 421.1370$, found: 421.1383; HPLC (Chiralpak ${ }^{\circledR} \mathrm{IC}$ column, ethanol/n-hexane = 2/8, flow rate $=1.0 \mathrm{~mL} / \mathrm{min}, 1=254 \mathrm{~nm}$ ) $\mathrm{t}^{\mathrm{R}}=16.7 \min$ (major), $18.3 \mathrm{~min}$ (minor).

\section{methyl lysinate (3ah) \\ $\mathrm{N}^{2}-((((9 H-f l u o r e n-9-y l) m e t h o x y)$ carbonyl)glycyl)-N6-(tert-butoxycarbonyl)-L-}<smiles>COC(=O)C(CCCCNC(=O)OCc1ccccc1)NC(=O)CNC(F)F</smiles>

3ah

Method A, Off-white solid; $52.7 \mathrm{mg}, 97 \%$ yield. ${ }^{1} \mathbf{H}$ NMR $\left(300 \mathrm{MHz}, \mathrm{CDCl}_{3}\right) \delta 7.74(\mathrm{~d}, J=7.4$ $\mathrm{Hz}, 2 \mathrm{H}), 7.59$ (d, $J=7.5 \mathrm{~Hz}, 2 \mathrm{H}), 7.38$ (t, $J=7.4 \mathrm{~Hz}, 2 \mathrm{H}), 7.28$ (td, $J=7.2,1.5 \mathrm{~Hz}, 2 \mathrm{H}), 7.01$ (d, $J=7.9 \mathrm{~Hz}, 1 \mathrm{H}), 5.92(\mathrm{~s}, 1 \mathrm{H}), 4.76(\mathrm{t}, J=6.0 \mathrm{~Hz}, 1 \mathrm{H}), 4.59(\mathrm{td}, J=7.7,4.8 \mathrm{~Hz}, 1 \mathrm{H}), 4.38(\mathrm{~d}, J=$ $7.1 \mathrm{~Hz}, 2 \mathrm{H}), 4.21(\mathrm{t}, J=7.2 \mathrm{~Hz}, 1 \mathrm{H}), 3.95$ (d, $J=5.5 \mathrm{~Hz}, 2 \mathrm{H}), 3.70$ (s, 3H), 3.04 (t, $J=6.6 \mathrm{~Hz}$, $2 \mathrm{H}), 1.82(\mathrm{dd}, J=9.7,5.5 \mathrm{~Hz}, 1 \mathrm{H}), 1.73-1.58(\mathrm{~m}, 1 \mathrm{H}), 1.42(\mathrm{~s}, 13 \mathrm{H}) .{ }^{13} \mathbf{C} \mathbf{N M R}\left(75 \mathrm{MHz}, \mathrm{CDCl}_{3}\right)$ $\delta 172.6,169.0,156.6,156.1,143.6,141.1,127.6,127.0,125.0,119.9,79.0,67.2,52.4,52.0,46.9$, 44.2, 39.8, 31.5, 29.3, 28.3, 22.3; HRMS (ESI) m/z: $[\mathrm{M}+\mathrm{H}]^{+}$calcd for $\mathrm{C}_{2} \mathrm{H}_{38} \mathrm{~N}_{3} \mathrm{O}_{7} 540.2704$, found: 540.2724; HPLC (Chiralpak ${ }^{\circledR}$ IC column, ethanol $/ \mathrm{n}$-hexane $=2 / 8$, flow rate $=1.0 \mathrm{~mL} / \mathrm{min}$, $1=254 \mathrm{~nm}) \mathrm{t}^{\mathrm{R}}=15.6 \min$ (major), $19.4 \min$ (minor).

methyl 2-(2-(((9H-fluoren-9-yl)methoxy)carbonyl)amino)acetamido)-2-methylpropanoate (3ai)<smiles>COC(=O)C(C)(C)NC(=O)CNC(F)F</smiles>

3ai

Method A, Off-white solid; $35.1 \mathrm{mg}, 89 \%$ yield. ${ }^{1} \mathbf{H}$ NMR $\left(300 \mathrm{MHz}, \mathrm{CDCl}_{3}\right) \delta 7.75(\mathrm{~d}, J=7.5$ $\mathrm{Hz}, 2 \mathrm{H}), 7.58(\mathrm{~d}, J=7.4 \mathrm{~Hz}, 2 \mathrm{H}), 7.38(\mathrm{t}, J=7.4 \mathrm{~Hz}, 2 \mathrm{H}), 7.35-7.18(\mathrm{~m}, 2 \mathrm{H}), 6.85(\mathrm{~s}, 1 \mathrm{H}), 5.74$ (s, 1H), $4.38(\mathrm{~d}, J=7.2 \mathrm{~Hz}, 2 \mathrm{H}), 4.20(\mathrm{t}, J=7.2 \mathrm{~Hz}, 1 \mathrm{H}), 3.88(\mathrm{~d}, J=5.3 \mathrm{~Hz}, 2 \mathrm{H}), 3.72(\mathrm{~s}, 3 \mathrm{H})$, $1.54(\mathrm{~s}, 6 \mathrm{H}) .{ }^{13} \mathrm{C}$ NMR $\left(75 \mathrm{MHz}, \mathrm{CDCl}_{3}\right) \delta 174.8,168.2,156.6,143.7,141.3,127.8,127.1,125.1$, 120.0, 67.3, 56.6, 52.8, 47.0, 44.5, 24.8; HRMS (ESI) m/z: $[\mathrm{M}+\mathrm{Na}]^{+}$calcd for $\mathrm{C}_{22} \mathrm{H}_{24} \mathrm{~N}_{2} \mathrm{O}_{5} \mathrm{Na}$ 419.1577, found: 419.1570 . 


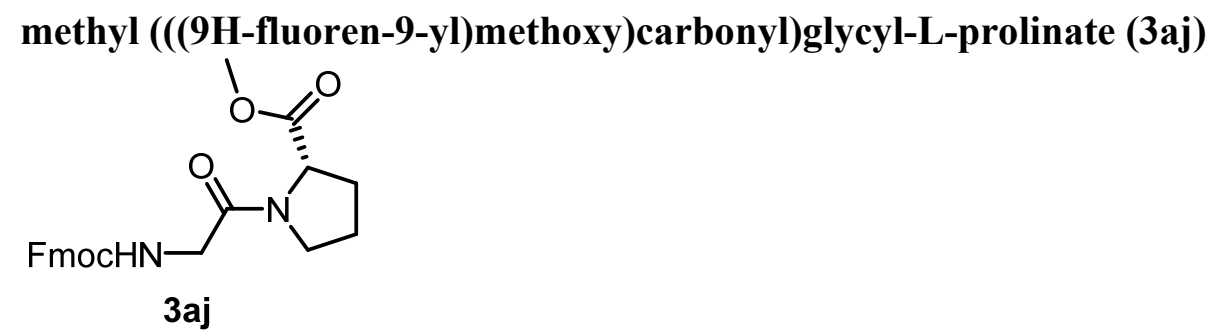

Method B, Colorless oil; $34.0 \mathrm{mg}, 83 \%$ yield. ${ }^{1} \mathbf{H}$ NMR $\left(300 \mathrm{MHz}, \mathrm{CDCl}_{3}\right) \delta 7.75(\mathrm{~d}, J=7.5 \mathrm{~Hz}$, 2H), 7.60 (d, $J=7.4 \mathrm{~Hz}, 2 \mathrm{H}), 7.39$ (t, $J=7.4 \mathrm{~Hz}, 2 \mathrm{H}), 7.29$ (dd, $J=14.1,6.6 \mathrm{~Hz}, 2 \mathrm{H}), 5.80$ (t, $J=$ $4.5 \mathrm{~Hz}, 1 \mathrm{H}), 4.57-4.49(\mathrm{~m}, 1 \mathrm{H}), 4.36(\mathrm{~d}, J=7.3 \mathrm{~Hz}, 2 \mathrm{H}), 4.22(\mathrm{t}, J=7.3 \mathrm{~Hz}, 1 \mathrm{H}), 4.04(\mathrm{t}, J=5.0$ $\mathrm{Hz}, 2 \mathrm{H}), 3.74(\mathrm{~s}, 3 \mathrm{H}), 3.59(\mathrm{~m}, 1 \mathrm{H}), 3.47$ (m, 1H), $2.24-1.89(\mathrm{~m}, 4 \mathrm{H}) ;{ }^{13} \mathbf{C} \mathbf{N M R}\left(75 \mathrm{MHz}, \mathrm{CDCl}_{3}\right)$ $\delta 172.2,166.9,156.1,143.8,143.7,141.1,127.6,127.0,125.1,119.8,67.0,58.8,52.8,52.3,47.0$, 45.8, 43.3, 43.1, 28.9, 24.5; HRMS (ESI) m/z: $[\mathrm{M}+\mathrm{Na}]^{+}$calcd for $\mathrm{C}_{23} \mathrm{H}_{24} \mathrm{~N}_{2} \mathrm{O}_{5} \mathrm{Na}$ 431.1577, found: 431.1591 .

methyl N-((((9H-fluoren-9-yl)methoxy)carbonyl)glycyl)-N-methyl-L-leucinate (3ak)<smiles>COC(=O)[C@H](CC(C)C)N(C)C(=O)CNC(F)F</smiles>

3ak

Method B, Colorless oil; $28.2 \mathrm{mg}, 64 \%$ yield. ${ }^{1} \mathbf{H}$ NMR $\left(300 \mathrm{MHz}, \mathrm{CDCl}_{3}\right) \delta 7.76(\mathrm{~d}, \mathrm{~J}=7.5 \mathrm{~Hz}$, 2H), $7.61(\mathrm{~d}, \mathrm{~J}=7.4 \mathrm{~Hz}, 2 \mathrm{H}), 7.47-7.35(\mathrm{~m}, 2 \mathrm{H}), 7.35-7.27(\mathrm{~m}, 2 \mathrm{H}), 5.87(\mathrm{~s}, 1 \mathrm{H}), 5.32(\mathrm{~d}, \mathrm{~J}=$ 9.9, $5.9 \mathrm{~Hz}, 1 \mathrm{H}), 4.38(\mathrm{~d}, \mathrm{~J}=7.2 \mathrm{~Hz}, 2 \mathrm{H}), 4.23$ (t, J = 7.2 Hz, 1H), 4.10 (d, J = 4.2 Hz, 2H), 3.73 $(\mathrm{d}, \mathrm{J}=5.9 \mathrm{~Hz}, 3 \mathrm{H}), 2.90(\mathrm{~s}, 3 \mathrm{H}), 1.94-1.64(\mathrm{~m}, 2 \mathrm{H}), 1.63-1.38(\mathrm{~m}, 1 \mathrm{H}), 0.95(\mathrm{t}, \mathrm{J}=6.9 \mathrm{~Hz}, 6 \mathrm{H})$. ${ }^{13} \mathbf{C}$ NMR $\left(75 \mathrm{MHz}, \mathrm{CDCl}_{3}\right) \delta 171.9,169.1,156.2,143.9,141.3,127.7,127.1,125.2,120.0,67.1$, 54.7, 52.4, 47.1, 43.0, 37.1, 30.1, 25.0, 23.2, 21.4; HRMS (ESI) $\mathrm{m} / \mathrm{z}:[\mathrm{M}+\mathrm{Na}]^{+}$calcd for $\mathrm{C}_{25} \mathrm{H}_{30} \mathrm{~N}_{2} \mathrm{O}_{5} \mathrm{Na} 461.2047$, found: 461.2034 .

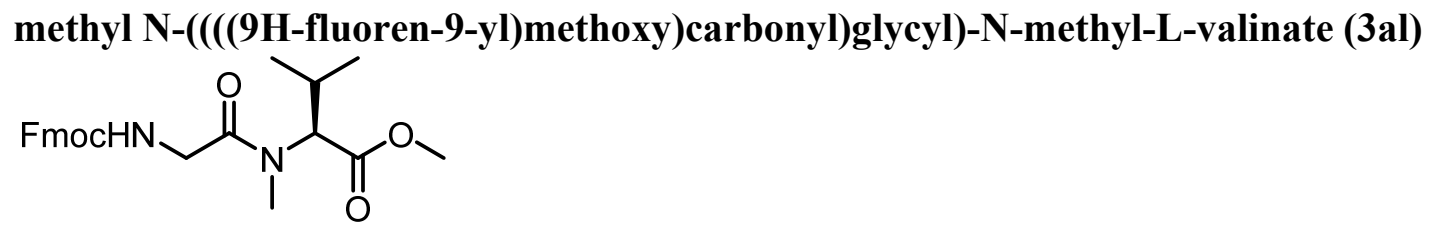


Method B, Colorless oil; $27.4 \mathrm{mg}, 65 \%$ yield. ${ }^{1} \mathbf{H}$ NMR $\left(300 \mathrm{MHz}, \mathrm{CDCl}_{3}\right) \delta 7.76(\mathrm{~d}, J=7.5 \mathrm{~Hz}$, 2H), $7.61(\mathrm{~d}, J=7.4 \mathrm{~Hz}, 2 \mathrm{H}), 7.40(\mathrm{t}, J=7.4 \mathrm{~Hz}, 2 \mathrm{H}), 7.31$ (t, $J=7.4,1.3 \mathrm{~Hz}, 2 \mathrm{H}), 6.00-5.76$ (m, 1H), $4.90(\mathrm{~d}, J=10.6 \mathrm{~Hz}, 1 \mathrm{H}), 4.38(\mathrm{~d}, J=7.3,2.8 \mathrm{~Hz}, 2 \mathrm{H}), 4.24$ (q, $J=7.6,5.5 \mathrm{~Hz}, 1 \mathrm{H}), 4.19$ $-4.00(\mathrm{~m}, 2 \mathrm{H}), 3.72(\mathrm{~d}, J=3.2 \mathrm{~Hz}, 3 \mathrm{H}), 2.95$ (d, $J=8.5 \mathrm{~Hz}, 3 \mathrm{H}), 2.39-2.12(\mathrm{~m}, 1 \mathrm{H}), 1.00$ (t, $J$ $=7.3 \mathrm{~Hz}, 3 \mathrm{H}), 0.89(\mathrm{~m}, J=8.8,6.6 \mathrm{~Hz}, 3 \mathrm{H}) .{ }^{13} \mathbf{C} \mathbf{N M R}\left(75 \mathrm{MHz}, \mathrm{CDCl}_{3}\right) \delta 171.1,169.8,169.1$, 168.5, 156.2, 143.9, 141.3, 127.7, 127.1, 125.2, 120.0, 67.2, 64.8, 61.8, 52.4, 52.0, 47.1, 43.0, 30.1, 28.7, 27.5, 27.4, 19.6, 19.5, 19.0, 18.6; HRMS (ESI) m/z: $[\mathrm{M}+\mathrm{Na}]^{+}$calcd for $\mathrm{C}_{24} \mathrm{H}_{28} \mathrm{~N}_{2} \mathrm{O}{ }_{5} \mathrm{Na}$ 447.1890, found: 447.1880 .

methyl (((9H-fluoren-9-yl)methoxy)carbonyl)-L-methionyl-L-alaninate (3ba)

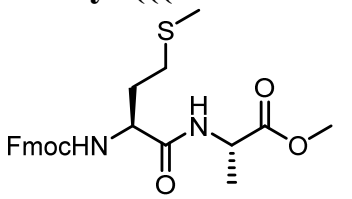

3ba

Method A, Off-white solid; $42.0 \mathrm{mg}, 92 \%$ yield. ${ }^{1} \mathbf{H}$ NMR $\left(300 \mathrm{MHz}, \mathrm{CDCl}_{3}\right) \delta 7.74(\mathrm{~d}, J=7.5$ $\mathrm{Hz}, 2 \mathrm{H}), 7.57$ (dd, $J=7.5,3.2 \mathrm{~Hz}, 2 \mathrm{H}), 7.37$ (t, $J=7.4 \mathrm{~Hz}, 2 \mathrm{H}), 7.28$ (t, $J=6.9 \mathrm{~Hz}, 2 \mathrm{H}), 6.98$ (d, $J=7.5 \mathrm{~Hz}, 1 \mathrm{H}), 5.86(\mathrm{~d}, J=8.4 \mathrm{~Hz}, 1 \mathrm{H}), 4.57$ (p, $J=7.1 \mathrm{~Hz}, 1 \mathrm{H}), 4.46(\mathrm{q}, J=7.2 \mathrm{~Hz}, 1 \mathrm{H}), 4.37$ (t, $J=7.0 \mathrm{~Hz}, 2 \mathrm{H}), 4.18(\mathrm{t}, J=7.1 \mathrm{~Hz}, 1 \mathrm{H}), 3.71(\mathrm{~s}, 3 \mathrm{H}), 2.59$ (t, $J=7.2 \mathrm{~Hz}, 2 \mathrm{H}), 2.09$ (s, 5H), $1.38(\mathrm{~d}, J=7.2 \mathrm{~Hz}, 3 \mathrm{H}) ;{ }^{13} \mathbf{C}$ NMR $\left(75 \mathrm{MHz}, \mathrm{CDCl}_{3}\right) \delta 172.9,170.9,156.0,143.7,143.6,141.1$, 127.6, 127.0, 125.0, 119.9, 119.9, 67.0, 53.4, 52.4, 48.0, 47.0, 31.8, 29.7, 17.9, 15.0; HRMS (ESI) $\mathrm{m} / \mathrm{z}:[\mathrm{M}+\mathrm{Na}]^{+}$calcd. for $\mathrm{C}_{24} \mathrm{H}_{28} \mathrm{~N}_{2} \mathrm{O}_{5} \mathrm{SNa} 479.1611$, found: 479.1632 .

tert-butyl (S)-4-((((9H-fluoren-9-yl)methoxy)carbonyl)amino)-5-(((S)-1-methoxy-1oxopropan-2-yl)amino)-5-oxopentanoate (3ca)<smiles>COCCOC(=O)N[C@@H](CCC(=O)OC)C(=O)NC(C)C(=O)OC</smiles>

Method A, Off-white solid; $49.6 \mathrm{mg}, 97 \%$ yield. ${ }^{1} \mathbf{H}$ NMR $\left(300 \mathrm{MHz}, \mathrm{CDCl}_{3}\right) \delta 7.74$ (d, $J=7.5$ $\mathrm{Hz}, 2 \mathrm{H}), 7.57(\mathrm{~d}, J=6.0 \mathrm{~Hz}, 2 \mathrm{H}), 7.37$ (t, $J=7.4 \mathrm{~Hz}, 2 \mathrm{H}), 7.33-7.22(\mathrm{~m}, 2 \mathrm{H}), 7.11(\mathrm{~d}, J=5.4$ $\mathrm{Hz}, 1 \mathrm{H}), 5.94$ (d, $J=9.3 \mathrm{~Hz}, 1 \mathrm{H}), 4.56$ (p, $J=7.3 \mathrm{~Hz}, 1 \mathrm{H}), 4.35$ (d, $J=7.3 \mathrm{~Hz}, 3 \mathrm{H}), 4.19$ (t, $J=$ $7.2 \mathrm{~Hz}, 1 \mathrm{H}), 3.72$ (s, 3H), 2.41 (dd, $J=8.7,3.8 \mathrm{~Hz}, 2 \mathrm{H}), 2.09$ (p, $J=6.9 \mathrm{~Hz}, 1 \mathrm{H}), 1.98$ (p, $J=7.3$ $\mathrm{Hz}, 1 \mathrm{H}), 1.45$ (s, 9H), 1.40 (d, $J=7.3 \mathrm{~Hz}, 3 \mathrm{H}) ;{ }^{13} \mathbf{C} \mathbf{N M R}\left(75 \mathrm{MHz}, \mathrm{CDCl}_{3}\right) \delta 172.9,172.8,171.0$, 
156.1, 143.7, 143.6, 141.1, 127.6, 126.9, 125.0, 119.8, 80.9, 67.0, 53.8, 52.3, 48.0, 47.0, 31.4, 28.3, 28.0, 17.9; HRMS (ESI) m/z: [M+Na] calcd for $\mathrm{C}_{28} \mathrm{H}_{34} \mathrm{~N}_{2} \mathrm{O}_{7} \mathrm{Na}$ 533.2258, found: 533.2272.

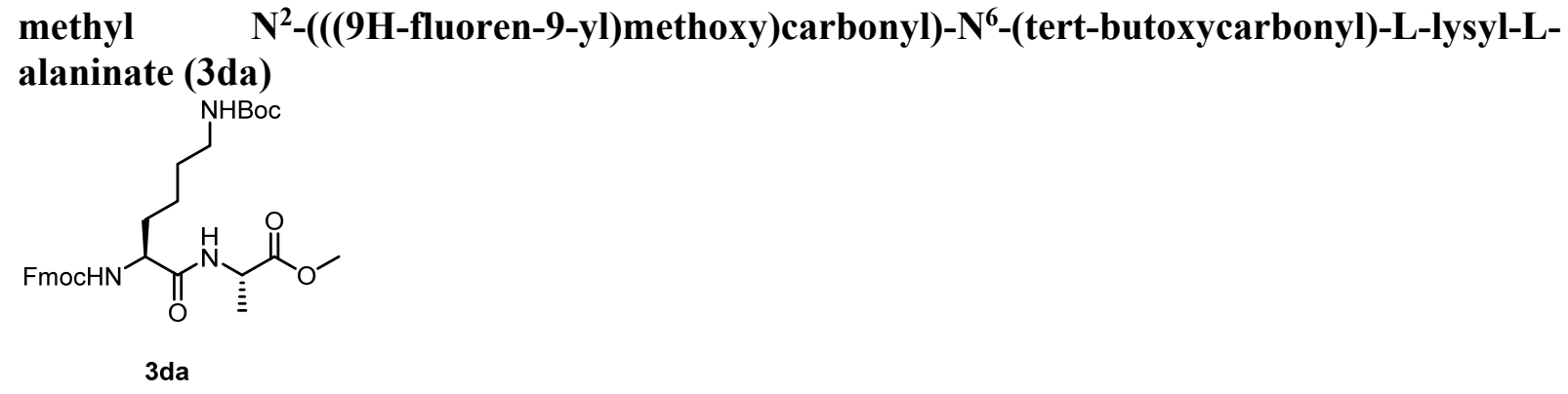

Method A, Off-white solid; $52.6 \mathrm{mg}, 95 \%$ yield. ${ }^{1} \mathbf{H}$ NMR $\left(300 \mathrm{MHz}, \mathrm{CDCl}_{3}\right) \delta 7.74(\mathrm{~d}, J=7.5$ $\mathrm{Hz}, 2 \mathrm{H}), 7.57$ (d, $J=6.8 \mathrm{~Hz}, 2 \mathrm{H}), 7.38$ (t, $J=7.5 \mathrm{~Hz}, 2 \mathrm{H}), 7.28$ (t, $J=6.2 \mathrm{~Hz}, 2 \mathrm{H}), 6.97$ (d, $J=$ $7.5 \mathrm{~Hz}, 1 \mathrm{H}), 5.81(\mathrm{~d}, J=8.2 \mathrm{~Hz}, 1 \mathrm{H}), 4.84(\mathrm{~s}, 1 \mathrm{H}), 4.56(\mathrm{p}, J=7.2 \mathrm{~Hz}, 1 \mathrm{H}), 4.35(\mathrm{~d}, J=7.2 \mathrm{~Hz}$, 2H), $4.23(\mathrm{~m}, 2 \mathrm{H}), 3.71(\mathrm{~s}, 3 \mathrm{H}), 3.10(\mathrm{~m}, 2 \mathrm{H}), 1.85(\mathrm{p}, J=6.7 \mathrm{~Hz}, 1 \mathrm{H}), 1.67(\mathrm{~m}, 1 \mathrm{H}), 1.39(\mathrm{~d}, J=$ $16.9 \mathrm{~Hz}, 17 \mathrm{H}) ;{ }^{13} \mathbf{C}$ NMR $\left(75 \mathrm{MHz}, \mathrm{CDCl}_{3}\right) \delta 173.1,171.5,156.1,143.7,143.6,141.1,127.6$, $127.0,125.0,119.9,79.0,67.0,54.4,52.4,48.0,47.0,39.8,32.3,29.4,28.3,22.2$, 17.8; HRMS (ESI) $\mathrm{m} / \mathrm{z}:[\mathrm{M}+\mathrm{Na}]^{+}$calcd for $\mathrm{C}_{30} \mathrm{H}_{39} \mathrm{~N}_{3} \mathrm{O}_{7} \mathrm{Na} 576.2680$, found: 576.2701 .

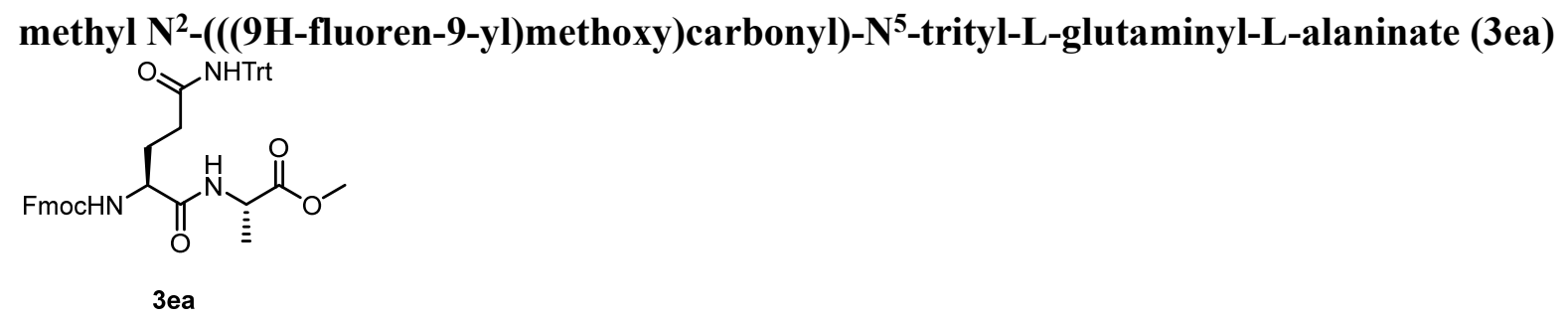

Method A, Off-white solid; $64.7 \mathrm{mg}, 93 \%$ yield. ${ }^{1} \mathbf{H}$ NMR $\left(300 \mathrm{MHz}, \mathrm{CDCl}_{3}\right) \delta 7.73$ (d, $J=7.5$ $\mathrm{Hz}, 2 \mathrm{H}), 7.56(\mathrm{~d}, J=7.4 \mathrm{~Hz}, 2 \mathrm{H}), 7.36$ (t, $J=7.4 \mathrm{~Hz}, 2 \mathrm{H}), 7.23$ (q, $J=7.3,6.7 \mathrm{~Hz}, 18 \mathrm{H}), 7.08$ (s, $1 \mathrm{H}), 5.96$ (d, $J=7.4 \mathrm{~Hz}, 1 \mathrm{H}), 4.44$ (q, $J=7.2 \mathrm{~Hz}, 1 \mathrm{H}), 4.33$ (d, $J=7.3 \mathrm{~Hz}, 2 \mathrm{H}), 4.17$ (t, $J=7.1$ $\mathrm{Hz}, 2 \mathrm{H}), 3.64(\mathrm{~s}, 3 \mathrm{H}), 2.51(\mathrm{~s}, 2 \mathrm{H}), 2.03(\mathrm{~m}, 2 \mathrm{H}), 1.26(\mathrm{~d}, J=7.3 \mathrm{~Hz}, 3 \mathrm{H}) ;{ }^{13} \mathbf{C} \mathbf{N M R}(75 \mathrm{MHz}$, $\left.\mathrm{CDCl}_{3}\right) \delta 173.0,171.9,171.0,156.0,144.4,143.8,143.7,141.2,141.1,128.6,127.9,127.6,127.0$, $126.9,125.1,119.8,70.5,66.9,53.4,52.3,48.1,47.0,33.1,29.6,17.3 ;$ HRMS (ESI) m/z: $[\mathrm{M}+\mathrm{Na}]^{+}$ calcd for $\mathrm{C}_{43} \mathrm{H}_{41} \mathrm{~N}_{3} \mathrm{O}_{6} \mathrm{Na} 718.2888$, found: 718.2895. 


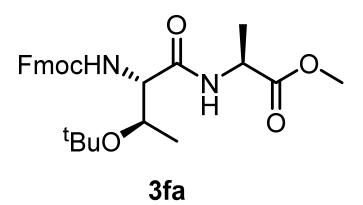

Method A, Off-white solid; $43.3 \mathrm{mg}, 90 \%$ yield. ${ }^{1} \mathbf{H}$ NMR $\left(300 \mathrm{MHz}, \mathrm{CDCl}_{3}\right) \delta 7.85-7.71(\mathrm{~m}$, $3 \mathrm{H}), 7.61(\mathrm{~d}, J=7.4 \mathrm{~Hz}, 2 \mathrm{H}), 7.39$ (t, $J=7.5 \mathrm{~Hz}, 2 \mathrm{H}), 7.35-7.24(\mathrm{~m}, 2 \mathrm{H}), 6.02$ (d, $J=5.1 \mathrm{~Hz}$, $1 \mathrm{H}), 4.52(\mathrm{p}, J=7.1 \mathrm{~Hz}, 1 \mathrm{H}), 4.38(\mathrm{~d}, J=7.2 \mathrm{~Hz}, 2 \mathrm{H}), 4.21(\mathrm{~m}, 3 \mathrm{H}), 3.76(\mathrm{~s}, 3 \mathrm{H}), 1.44$ (d, $J=7.2$ $\mathrm{Hz}, 3 \mathrm{H}), 1.31(\mathrm{~s}, 9 \mathrm{H}), 1.11(\mathrm{~d}, J=6.2 \mathrm{~Hz}, 3 \mathrm{H}) ;{ }^{13} \mathbf{C} \mathbf{N M R}\left(75 \mathrm{MHz}, \mathrm{CDCl}_{3}\right) \delta 172.9,169.1,155.9$, 143.8, 143.6, 141.2, 141.2, 127.6, 127.0, 125.1, 119.9, 119.9, 75.5, 66.9, 66.6, 58.3, 52.3, 48.3, 47.1, 28.1, 18.1, 16.4; HRMS (ESI) m/z: $[\mathrm{M}+\mathrm{Na}]^{+}$calcd for $\mathrm{C}_{27} \mathrm{H}_{34} \mathrm{~N}_{2} \mathrm{O}_{6} \mathrm{Na}$ 505.2309, found: 505.2333 .

methyl $N^{\alpha}$-(((9H-fluoren-9-yl)methoxy)carbonyl)-N ${ }^{\tau}$-trityl-L-histidyl-L-alaninate (3ga)

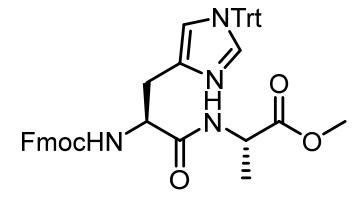

$3 g a$

Method A, Off-white solid; $63.6 \mathrm{mg}, 90 \%$ yield. ${ }^{1} \mathbf{H}$ NMR $\left(300 \mathrm{MHz}, \mathrm{CDCl}_{3}\right) \delta 7.75$ (d, $J=7.5$ $\mathrm{Hz}, 3 \mathrm{H}), 7.61(\mathrm{~d}, J=7.5 \mathrm{~Hz}, 2 \mathrm{H}), 7.34(\mathrm{~m}, 14 \mathrm{H}), 7.17-7.00(\mathrm{~m}, 6 \mathrm{H}), 6.76(\mathrm{~d}, J=7.1 \mathrm{~Hz}, 1 \mathrm{H})$, $6.68(\mathrm{~s}, 1 \mathrm{H}), 4.54(\mathrm{~m}, 2 \mathrm{H}), 4.34(\mathrm{~d}, J=7.1 \mathrm{~Hz}, 2 \mathrm{H}), 4.22(\mathrm{t}, J=7.5 \mathrm{~Hz}, 1 \mathrm{H}), 3.66(\mathrm{~s}, 3 \mathrm{H}), 3.03(\mathrm{q}$, $J=8.5,7.4 \mathrm{~Hz}, 2 \mathrm{H}), 2.69(\mathrm{~s}, 1 \mathrm{H}), 1.34(\mathrm{~d}, J=7.2 \mathrm{~Hz}, 3 \mathrm{H}) ;{ }^{13} \mathbf{C ~ N M R}\left(75 \mathrm{MHz}, \mathrm{CDCl}_{3}\right) \delta 173.0$, $171.0,156.2,143.9,143.8,142.2,141.1,138.4,136.6,129.7,128.0,128.0,127.6,127.0,125.2$, 125.2, 119.8, 119.5, 75.3, 67.1, 54.8, 52.3, 48.2, 47.0, 30.6, 18.1; HRMS (ESI) m/z: $[\mathrm{M}+\mathrm{H}]^{+}$calcd for $\mathrm{C}_{44} \mathrm{H}_{41} \mathrm{~N}_{4} \mathrm{O}_{5}$ 705.3071, found: 705.3081.

tert-butyl (S)-3-(((9H-fluoren-9-yl)methoxy)carbonyl)amino)-4-(((S)-1-methoxy-1oxopropan-2-yl)amino)-4-oxobutanoate (3ha)

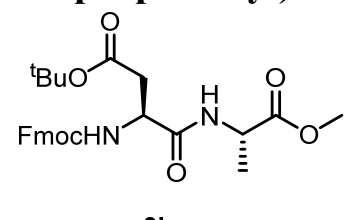

3ha

Method A, Off-white solid; $46.1 \mathrm{mg}, 93 \%$ yield. ${ }^{1} \mathbf{H}$ NMR $\left(300 \mathrm{MHz}, \mathrm{CDCl}_{3}\right) \delta 7.74(\mathrm{~d}, J=7.5$ $\mathrm{Hz}, 2 \mathrm{H}), 7.57$ (d, $J=6.0 \mathrm{~Hz}, 2 \mathrm{H}), 7.37$ (t, $J=7.4 \mathrm{~Hz}, 2 \mathrm{H}), 7.33-7.22(\mathrm{~m}, 2 \mathrm{H}), 7.11$ (d, $J=5.4$ $\mathrm{Hz}, 1 \mathrm{H}), 5.94(\mathrm{~d}, J=9.3 \mathrm{~Hz}, 1 \mathrm{H}), 4.56$ (p, $J=7.3 \mathrm{~Hz}, 1 \mathrm{H}), 4.35(\mathrm{~d}, J=7.3 \mathrm{~Hz}, 3 \mathrm{H}), 4.19$ (t, $J=$ 
$7.2 \mathrm{~Hz}, 1 \mathrm{H}), 3.72(\mathrm{~s}, 3 \mathrm{H}), 2.41(\mathrm{dd}, J=8.7,3.8 \mathrm{~Hz}, 2 \mathrm{H}), 2.09$ (p, $J=6.9 \mathrm{~Hz}, 1 \mathrm{H}), 1.98$ (p, $J=7.3$ $\mathrm{Hz}, 1 \mathrm{H}), 1.45$ (s, 9H), 1.40 (d, $J=7.3 \mathrm{~Hz}, 3 \mathrm{H}) ;{ }^{13} \mathbf{C} \mathbf{N M R}\left(75 \mathrm{MHz}, \mathrm{CDCl}_{3}\right) \delta 172.9,172.8,171.0$, 156.1, 143.7, 143.6, 141.1, 127.6, 126.9, 125.0, 119.8, 80.9, 67.0, 53.8, 52.3, 48.0, 47.0, 31.4, 28.3, 28.0, 17.9; HRMS (ESI) m/z: [M+Na] $]^{+}$calcd for $\mathrm{C}_{28} \mathrm{H}_{34} \mathrm{~N}_{2} \mathrm{O}_{7} \mathrm{Na}$ 533.2258, found: 533.2272.

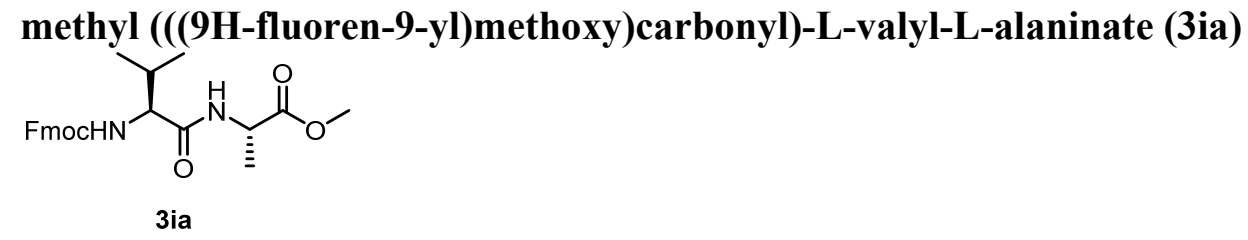

3ia

Method A, Off-white solid; $38.6 \mathrm{mg}, 91 \%$ yield. ${ }^{1} \mathbf{H}$ NMR $\left(300 \mathrm{MHz}, \mathrm{CDCl}_{3}\right) \delta 7.74(\mathrm{~d}, J=7.5$ $\mathrm{Hz}, 2 \mathrm{H}), 7.57$ (d, $J=5.4 \mathrm{~Hz}, 2 \mathrm{H}), 7.38$ (t, $J=7.1 \mathrm{~Hz}, 2 \mathrm{H}), 7.28$ (t, $J=7.5 \mathrm{~Hz}, 2 \mathrm{H}), 6.79$ (d, $J=$ $7.4 \mathrm{~Hz}, 1 \mathrm{H}), 5.69$ (d, $J=9.1 \mathrm{~Hz}, 1 \mathrm{H}), 4.58$ (p, $J=7.3 \mathrm{~Hz}, 1 \mathrm{H}), 4.48-4.37$ (m, 1H), $4.36-4.25$ $(\mathrm{m}, 1 \mathrm{H}), 4.19(\mathrm{t}, J=7.2 \mathrm{~Hz}, 1 \mathrm{H}), 4.14-4.03(\mathrm{~m}, 1 \mathrm{H}), 3.71(\mathrm{~s}, 3 \mathrm{H}), 2.08(\mathrm{~m}, 1 \mathrm{H}), 1.38(\mathrm{~d}, J=7.3$ $\mathrm{Hz}, 3 \mathrm{H}), 0.97(\mathrm{t}, J=7.5 \mathrm{~Hz}, 6 \mathrm{H}) ;{ }^{13} \mathbf{C} \mathbf{N M R}\left(75 \mathrm{MHz}, \mathrm{CDCl}_{3}\right) \delta 173.1,171.0,156.4,143.8,143.7$, 141.2, 127.6, 127.0, 125.1, 125.0, 119.9, 119.9, 67.0, 60.1, 52.4, 47.9, 47.0, 31.4, 19.0, 18.0, 17.9; HRMS (ESI) m/z: [M+Na] $]^{+}$calcd for $\mathrm{C}_{24} \mathrm{H}_{28} \mathrm{~N}_{2} \mathrm{O}_{5} \mathrm{Na} 447.1890$, found: 447.1909.

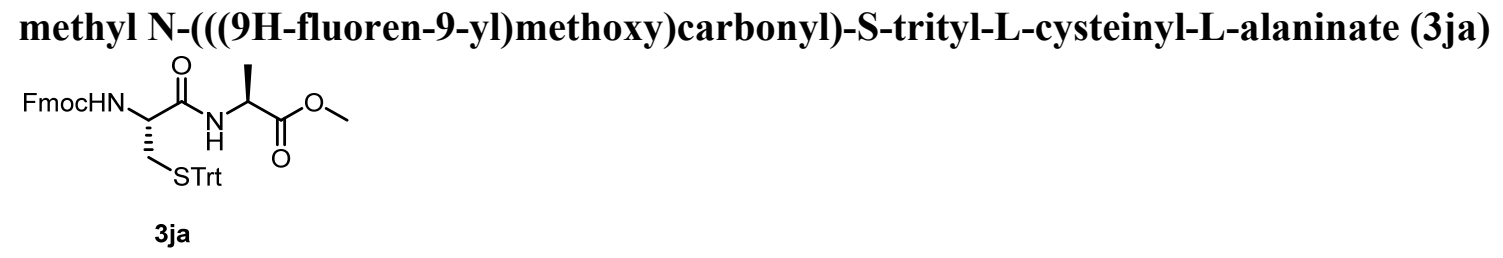

Method A, Off-white solid; $62.3 \mathrm{mg}, 93 \%$ yield. ${ }^{1} \mathbf{H}$ NMR $\left(300 \mathrm{MHz}, \mathrm{CDCl}_{3}\right) \delta 7.73(\mathrm{t}, J=6.7 \mathrm{~Hz}$, 2H), $7.56(\mathrm{~d}, J=7.5 \mathrm{~Hz}, 2 \mathrm{H}), 7.47-7.32(\mathrm{~m}, 8 \mathrm{H}), 7.23(\mathrm{~m}, 11 \mathrm{H}), 6.40(\mathrm{~d}, J=7.5 \mathrm{~Hz}, 1 \mathrm{H}), 5.15$ $(\mathrm{d}, J=8.0 \mathrm{~Hz}, 1 \mathrm{H}), 4.46(\mathrm{p}, J=7.2 \mathrm{~Hz}, 1 \mathrm{H}), 4.36(\mathrm{dd}, J=7.1,2.2 \mathrm{~Hz}, 2 \mathrm{H}), 4.18$ (t, $J=6.9 \mathrm{~Hz}$, $1 \mathrm{H}), 3.77$ (q, $J=7.7 \mathrm{~Hz}, 1 \mathrm{H}), 3.66(\mathrm{~s}, 3 \mathrm{H}), 2.71(\mathrm{dd}, J=13.3,7.8 \mathrm{~Hz}, 1 \mathrm{H}), 2.60$ (dd, $J=13.3,5.3$ $\mathrm{Hz}, 1 \mathrm{H}), 1.33(\mathrm{~d}, J=7.1 \mathrm{~Hz}, 3 \mathrm{H}) ;{ }^{13} \mathbf{C} \mathbf{N M R}\left(75 \mathrm{MHz}, \mathrm{CDCl}_{3}\right) \delta 172.6,169.6,155.8,144.3,143.6$, 143.6, 141.2, 129.5, 128.0, 127.7, 127.0, 126.8, 125.0, 119.9, 67.2, 67.0, 53.8, 52.4, 48.1, 47.0, 33.8, 18.1; HRMS (ESI) m/z: [M+Na $]^{+}$calcd for $\mathrm{C}_{41} \mathrm{H}_{38} \mathrm{~N}_{2} \mathrm{O}_{5} \mathrm{SNa}$ 693.2394, found: 693.2402.

methyl N-(((9H-fluoren-9-yl)methoxy)carbonyl)-O-(tert-butyl)-L-seryl-L-alaninate (3ka)<smiles>COC(=O)C(C)NC(=O)[C@H](CO)NCCO</smiles>

$3 k a$ 
Method A, Off-white solid; $44.9 \mathrm{mg}, 96 \%$ yield. ${ }^{1} \mathbf{H}$ NMR $\left(300 \mathrm{MHz}, \mathrm{CDCl}_{3}\right) \delta 7.75$ (d, $J=7.4$ Hz, 2H), 7.60 (d, $J=7.2 \mathrm{~Hz}, 2 \mathrm{H}), 7.48$ (d, $J=7.1 \mathrm{~Hz}, 1 \mathrm{H}), 7.39$ (t, $J=7.4 \mathrm{~Hz}, 2 \mathrm{H}), 7.29$ (m, 2H), $5.83(\mathrm{~d}, J=5.4 \mathrm{~Hz}, 1 \mathrm{H}), 4.59(\mathrm{p}, J=7.1 \mathrm{~Hz}, 1 \mathrm{H}), 4.39(\mathrm{~d}, J=7.1 \mathrm{~Hz}, 2 \mathrm{H}), 4.22$ (t, $J=7.1 \mathrm{~Hz}$, 2H), $3.81(\mathrm{dd}, J=8.3,3.9 \mathrm{~Hz}, 1 \mathrm{H}), 3.74(\mathrm{~s}, 3 \mathrm{H}), 3.39$ (t, $J=8.6 \mathrm{~Hz}, 1 \mathrm{H}), 1.42$ (d, $J=7.2 \mathrm{~Hz}, 3 \mathrm{H})$, $1.24(\mathrm{~s}, 9 \mathrm{H}) ;{ }^{13} \mathrm{C}$ NMR $\left(75 \mathrm{MHz}, \mathrm{CDCl}_{3}\right) \delta 172.9,169.8,155.9,143.8,143.6,141.2,127.6,127.0$, 125.0, 119.9, 74.3, 67.0, 61.7, 54.0, 52.3, 48.2, 47.0, 27.3, 18.3; HRMS (ESI) m/z: $[\mathrm{M}+\mathrm{Na}]^{+}$calcd for $\mathrm{C}_{16} \mathrm{H}_{17} \mathrm{NONa} 262.1202$, found: 262.1201 .

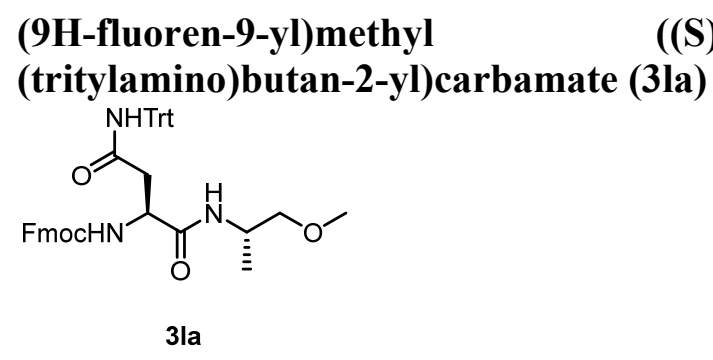

Method A, Off-white solid; $56.3 \mathrm{mg}, 84 \%$ yield. ${ }^{1} \mathbf{H}$ NMR $\left(300 \mathrm{MHz}, \mathrm{CDCl}_{3}\right) \delta 7.74(\mathrm{~d}, J=7.5$ $\mathrm{Hz}, 2 \mathrm{H}), 7.56(\mathrm{~d}, J=7.4 \mathrm{~Hz}, 2 \mathrm{H}), 7.38$ (t, $J=7.4 \mathrm{~Hz}, 2 \mathrm{H}), 7.31-7.16(\mathrm{~m}, 18 \mathrm{H}), 7.00$ (s, 1H), 6.49 $(\mathrm{d}, J=8.1 \mathrm{~Hz}, 1 \mathrm{H}), 4.58(\mathrm{~s}, 1 \mathrm{H}), 4.50-4.28(\mathrm{~m}, 3 \mathrm{H}), 4.18(\mathrm{t}, J=7.1 \mathrm{~Hz}, 1 \mathrm{H}), 3.69$ (s, 3H), 3.10 $(\mathrm{dd}, J=15.7,3.7 \mathrm{~Hz}, 1 \mathrm{H}), 2.65(\mathrm{dd}, J=15.8,6.3 \mathrm{~Hz}, 1 \mathrm{H}), 1.30(\mathrm{~d}, J=7.2 \mathrm{~Hz}, 3 \mathrm{H}) ;{ }^{13} \mathbf{C}$ NMR $(75$ $\left.\mathrm{MHz}, \mathrm{CDCl}_{3}\right) \delta 172.8,170.6,170.5,156.1,144.1,143.7,143.6,141.2,141.2,128.6,127.9,127.6$, 127.1, 127.0, 125.1, 119.9, 70.8, 67.2, 52.3, 51.1, 48.3, 47.0, 38.3, 17.6; HRMS (ESI) m/z: $[\mathrm{M}+\mathrm{Na}]^{+}$calcd for $\mathrm{C}_{42} \mathrm{H}_{39} \mathrm{~N}_{3} \mathrm{O}_{6} \mathrm{Na}$ 704.2731, found: 704.2720.

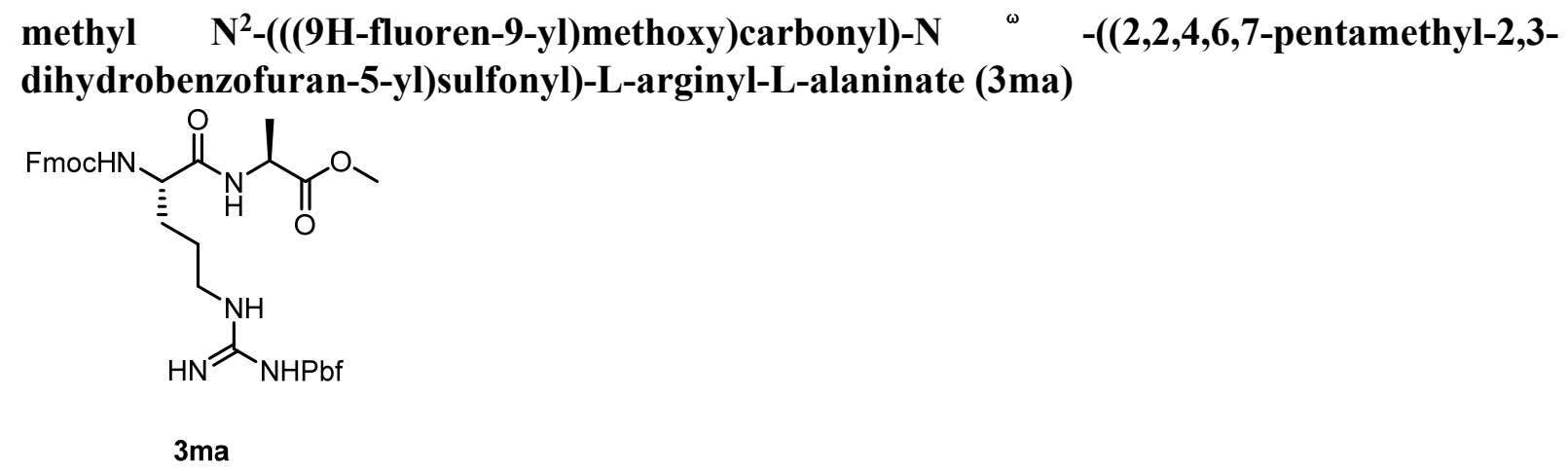

Method A, Off-white solid; $64.1 \mathrm{mg}, 87 \%$ yield. ${ }^{1} \mathbf{H}$ NMR $\left(300 \mathrm{MHz}, \mathrm{CDCl}_{3}\right) \delta 7.70$ (d, $J=7.6$ $\mathrm{Hz}, 3 \mathrm{H}), 7.54$ (d, $J=7.5 \mathrm{~Hz}, 2 \mathrm{H}), 7.33$ (t, $J=7.5 \mathrm{~Hz}, 2 \mathrm{H}), 7.23$ (q, $J=7.4,6.5 \mathrm{~Hz}, 2 \mathrm{H}), 6.41$ (s, 2H), $6.30(\mathrm{~s}, 1 \mathrm{H}), 6.21(\mathrm{~d}, J=8.1 \mathrm{~Hz}, 1 \mathrm{H}), 4.44(\mathrm{~m}, 2 \mathrm{H}), 4.28(\mathrm{~d}, J=7.5 \mathrm{~Hz}, 2 \mathrm{H}), 4.12$ (t, $J=7.2$ $\mathrm{Hz}, 1 \mathrm{H}), 3.65$ (s, 4H), 3.27 (d, $J=36.6 \mathrm{~Hz}, 2 \mathrm{H}), 3.03$ (d, $J=7.5 \mathrm{~Hz}, 1 \mathrm{H}), 2.88(\mathrm{~s}, 2 \mathrm{H}), 2.54$ (d, 
$J=25.9 \mathrm{~Hz}, 6 \mathrm{H}), 2.05(\mathrm{~s}, 3 \mathrm{H}), 1.91(\mathrm{~s}, 1 \mathrm{H}), 1.60(\mathrm{~s}, 3 \mathrm{H}), 1.45-1.31(\mathrm{~m}, 14 \mathrm{H}) ;{ }^{13} \mathrm{C}$ NMR $(75$ $\left.\mathrm{MHz}, \mathrm{CDCl}_{3}\right) \delta 173.5,158.7,156.4,156.3,143.7,143.6,141.1,141.0,138.2,132.5,132.1,127.6$, $126.9,125.0,124.6,119.8,117.5,86.3,67.0,52.3,48.3,46.9,43.0,28.4,19.3,17.9,17.1,12.4$; HRMS (ESI) m/z: [M+Na] $]^{+}$calcd for $\mathrm{C}_{38} \mathrm{H}_{47} \mathrm{~N}_{5} \mathrm{O}_{8} \mathrm{SNa} 756.3038$, found: 756.3030 .

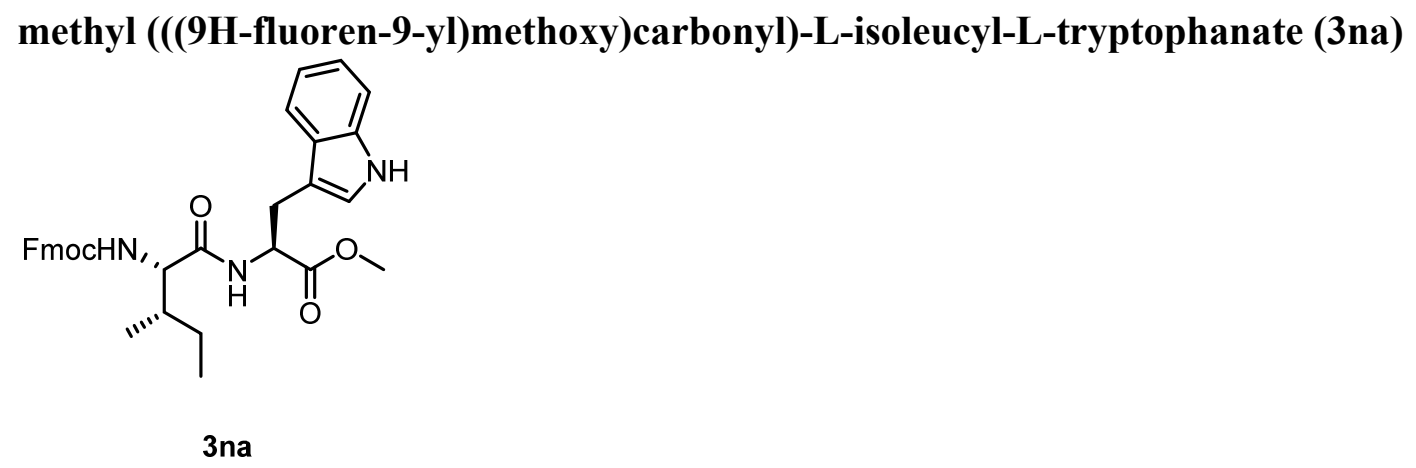

Method A, Off-white solid; $49.7 \mathrm{mg}, 90 \%$ yield. ${ }^{1} \mathbf{H}$ NMR $\left(300 \mathrm{MHz}, \mathrm{CDCl}_{3}\right) \delta 8.26$ (d, $J=7.8$ Hz, 1H), 7.73 (dd, $J=7.6,3.0 \mathrm{~Hz}, 2 \mathrm{H}), 7.55$ (d, $J=7.5 \mathrm{~Hz}, 1 \mathrm{H}), 7.51-7.31$ (m, 4H), $7.32-7.19$ (m, 3H), $7.16-6.99(\mathrm{~m}, 2 \mathrm{H}), 6.84(\mathrm{~m}, 2 \mathrm{H}), 5.52$ (t, $J=8.8 \mathrm{~Hz}, 1 \mathrm{H}), 5.00-4.87(\mathrm{~m}, 1 \mathrm{H}), 4.34-$ $4.16(\mathrm{~m}, 2 \mathrm{H}), 4.13-3.94$ (m, 2H), 3.59 (s, 3H), 3.25 (m, $J=14.8,5.4 \mathrm{~Hz}, 2 \mathrm{H}), 1.78$ (dd, $J=6.5$, $3.3 \mathrm{~Hz}, 1 \mathrm{H}), 1.39(\mathrm{~s}, 1 \mathrm{H}), 1.12-0.94(\mathrm{~m}, 1 \mathrm{H}), 0.93-0.60(\mathrm{~m}, 6 \mathrm{H}) ;{ }^{13} \mathbf{C} \mathbf{N M R}\left(75 \mathrm{MHz}, \mathrm{CDCl}_{3}\right)$ $\delta 172.0,171.5,156.4,144.0,143.7,141.3,136.1,127.7,127.4,127.1,125.1,123.2,122.2,120.0$, 120.0, 119.6, 118.4, 111.4, 109.3, 67.0, 59.3, 52.7, 52.4, 47.1, 37.9, 27.7, 24.5, 15.3, 11.4; HRMS (ESI) $\mathrm{m} / \mathrm{z}:[\mathrm{M}+\mathrm{Na}]^{+}$calcd for $\mathrm{C}_{33} \mathrm{H}_{35} \mathrm{~N}_{3} \mathrm{O}_{5} \mathrm{Na} 576.2509$, found: 576.2484 .

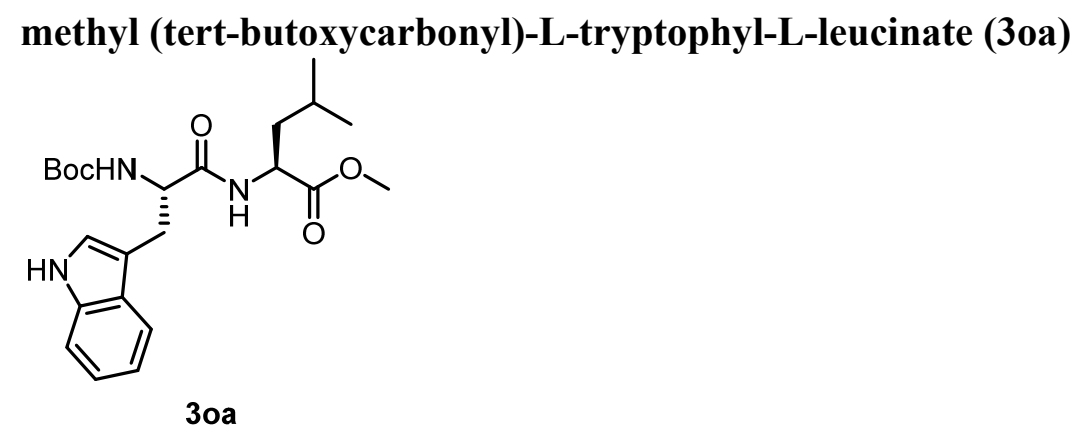

Method A, Off-white solid; $40.8 \mathrm{mg}, 95 \%$ yield. ${ }^{1} \mathbf{H}$ NMR $\left(300 \mathrm{MHz}, \mathrm{CDCl}_{3}\right) \delta 8.43(\mathrm{~s}, 1 \mathrm{H}), 7.64$ $(\mathrm{d}, J=7.8 \mathrm{~Hz}, 1 \mathrm{H}), 7.34(\mathrm{~d}, J=8.0 \mathrm{~Hz}, 1 \mathrm{H}), 7.26-6.87(\mathrm{~m}, 3 \mathrm{H}), 6.29$ (d, $J=8.1 \mathrm{~Hz}, 1 \mathrm{H}), 5.22$ (d, $J=7.4 \mathrm{~Hz}, 1 \mathrm{H}), 4.51$ (q, $J=11.0,9.8,4.4 \mathrm{~Hz}, 2 \mathrm{H}), 3.63$ (s, 3H), 3.24 (d, $J=14.5,8.0,7.3 \mathrm{~Hz}$, 2H), $1.43(\mathrm{~s}, 12 \mathrm{H}), 0.84(\mathrm{~d}, J=6.1,4.4 \mathrm{~Hz}, 6 \mathrm{H}) ;{ }^{13} \mathbf{C ~ N M R}\left(75 \mathrm{MHz}, \mathrm{CDCl}_{3}\right) \delta 172.9,171.5,155.5$, 
$136.2,127.5,123.4,122.1,119.6,118.8,111.2,110.4,80.1,55.1,52.2,50.8,41.5,28.3,24.6,22.7$, 21.9; HRMS (ESI) m/z: [M+Na $]^{+}$calcd for $\mathrm{C}_{23} \mathrm{H}_{33} \mathrm{~N}_{3} \mathrm{O}_{5} \mathrm{Na}$ 454.2312, found: 454.2322.

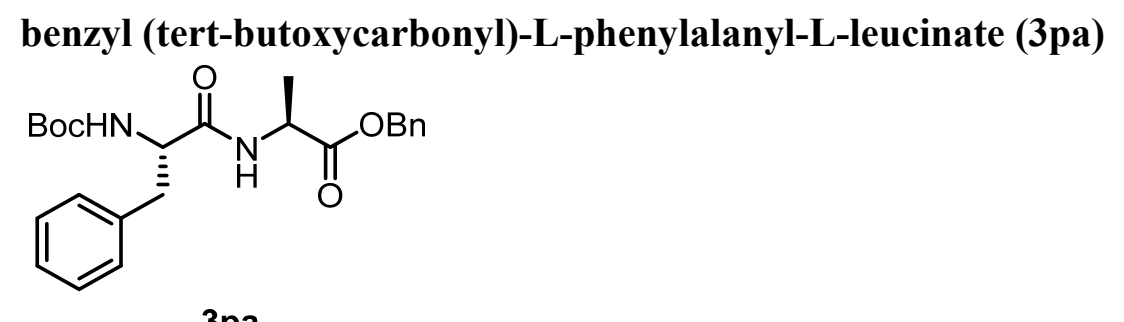

Method A, Off-white solid; $37.9 \mathrm{mg}, 89 \%$ yield. ${ }^{1} \mathbf{H}$ NMR $\left(300 \mathrm{MHz}, \mathrm{CDCl}_{3}\right) \delta 7.49-7.29$ (m, $5 \mathrm{H}), 7.29-7.10(\mathrm{~m}, 5 \mathrm{H}), 6.66(\mathrm{~d}, J=7.2 \mathrm{~Hz}, 1 \mathrm{H}), 5.14(\mathrm{~s}, 3 \mathrm{H}), 4.55$ (q, $J=7.2 \mathrm{~Hz}, 1 \mathrm{H}), 4.48-$ $4.28(\mathrm{~m}, 1 \mathrm{H}), 3.04(\mathrm{~d}, J=6.1 \mathrm{~Hz}, 2 \mathrm{H}), 1.48-1.26(\mathrm{~m}, 12 \mathrm{H}) ;{ }^{13} \mathbf{C ~ N M R}\left(75 \mathrm{MHz}, \mathrm{CDCl}_{3}\right) \delta 172.3$, $170.9,155.4$, 136.5, 135.3, 129.3, 128.6, 128.6, 128.4, 128.1, 126.9, 80.1, 67.1, 48.2, 38.4, 28.2, 18.3; HRMS (ESI) m/z: [M+Na $]^{+}$calcd for $\mathrm{C}_{24} \mathrm{H}_{30} \mathrm{~N}_{2} \mathrm{O}_{5} \mathrm{Na} 449.2047$, found: 449.2060 .

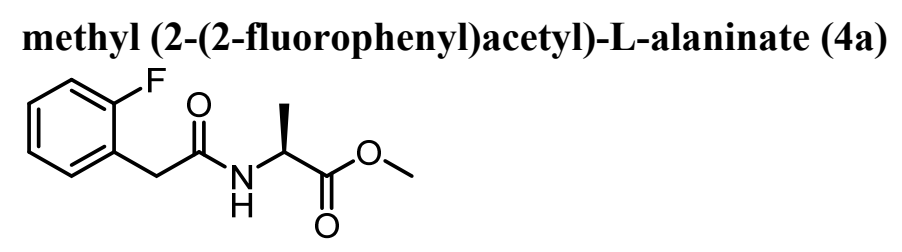

Method B, Off-white solid; $16.5 \mathrm{mg}, 69 \%$ yield. ${ }^{1} \mathbf{H}$ NMR (300 MHz, $\left.\mathrm{CDCl}_{3}\right) \delta 7.30(\mathrm{M}, 2 \mathrm{H}), 7.20$ $-7.00(\mathrm{~m}, 2 \mathrm{H}), 6.31-6.08(\mathrm{~m}, 1 \mathrm{H}), 4.59$ (p, $J=7.2 \mathrm{~Hz}, 1 \mathrm{H}), 3.72(\mathrm{~s}, 3 \mathrm{H}), 3.61(\mathrm{~s}, 2 \mathrm{H}), 1.37$ (d, $J$ $=7.2 \mathrm{~Hz}, 3 \mathrm{H}) ;{ }^{13} \mathbf{C}$ NMR $\left(75 \mathrm{MHz}, \mathrm{CDCl}_{3}\right) \delta 173.4,169.3,162.6,159.3,131.6,131.6,129.4$, 129.3, 124.6, 124.5, 121.9, 121.7, 115.7, 115.4, 52.5, 48.2, 36.7, 36.6, 18.3; HRMS (ESI) m/z: $[\mathrm{M}+\mathrm{Na}]^{+}$calcd for $\mathrm{C}_{16} \mathrm{H}_{17} \mathrm{NONa} 262.1202$, found: 262.1201 .

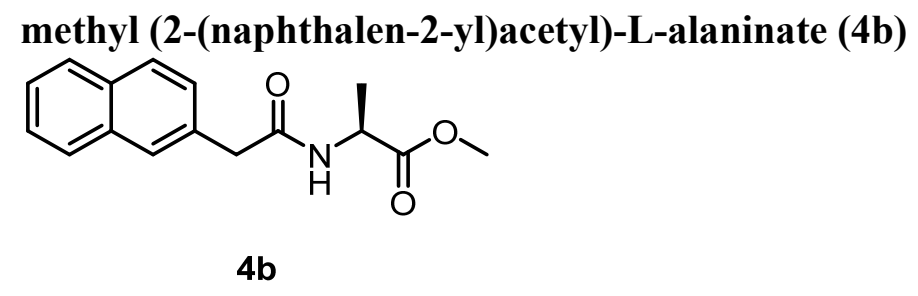

Method B, Off-white solid; $21.7 \mathrm{mg}, 80 \%$ yield. ${ }^{1} \mathbf{H}$ NMR $\left(300 \mathrm{MHz}, \mathrm{CDCl}_{3}\right) \delta 7.89-7.78(\mathrm{~m}$, $3 \mathrm{H}), 7.73(\mathrm{~s}, 1 \mathrm{H}), 7.56-7.43(\mathrm{~m}, 2 \mathrm{H}), 7.39(\mathrm{dd}, J=8.4,1.5 \mathrm{~Hz}, 1 \mathrm{H}), 6.13(\mathrm{~d}, J=6.5 \mathrm{~Hz}, 1 \mathrm{H})$, 4.60 (p, $J=7.2 \mathrm{~Hz}, 1 \mathrm{H}), 3.75(\mathrm{~s}, 2 \mathrm{H}), 3.69$ (s, 3H), 1.32 (d, $J=7.2 \mathrm{~Hz}, 3 \mathrm{H}) ;{ }^{13} \mathbf{C}$ NMR $(75 \mathrm{MHz}$, 
$\left.\mathrm{CDCl}_{3}\right) \delta 173.4,170.5,133.6,132.5,132.0,128.8,128.3,127.7,127.7,127.2,126.4,126.0,52.5$, 48.1, 43.7, 18.3; HRMS (ESI) m/z: [M+Na] $]^{+}$calcd for $\mathrm{C}_{16} \mathrm{H}_{17} \mathrm{NO}_{3} \mathrm{Na} 294.1101$, found: 294.1107 .

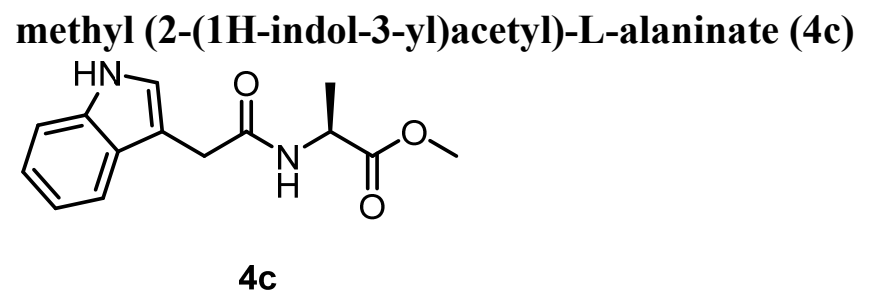

Method B, Off-white solid; $19.9 \mathrm{mg}, 77 \%$ yield. ${ }^{1} \mathbf{H}$ NMR $\left(300 \mathrm{MHz}, \mathrm{CDCl}_{3}\right) \delta 8.72(\mathrm{~s}, 1 \mathrm{H}), 7.56$ $(\mathrm{d}, J=7.8 \mathrm{~Hz}, 1 \mathrm{H}), 7.37$ (d, $J=8.0 \mathrm{~Hz}, 1 \mathrm{H}), 7.28-7.02(\mathrm{~m}, 3 \mathrm{H}), 6.31(\mathrm{~d}, J=7.3 \mathrm{~Hz}, 1 \mathrm{H}), 4.60$ $(\mathrm{p}, J=7.3 \mathrm{~Hz}, 1 \mathrm{H}), 3.75(\mathrm{~s}, 2 \mathrm{H}), 3.65(\mathrm{~s}, 3 \mathrm{H}), 1.28(\mathrm{~d}, J=7.2 \mathrm{~Hz}, 3 \mathrm{H}) ;{ }^{13} \mathbf{C} \mathbf{N M R}\left(75 \mathrm{MHz}, \mathrm{CDCl}_{3}\right)$ $\delta 173.3,171.3,136.3,126.9,123.9,122.3,119.7,118.5,111.4,108.2,52.3,48.0,33.2,18.0$; HRMS (ESI) m/z: [M+Na] $]^{+}$calcd for $\mathrm{C}_{14} \mathrm{H}_{16} \mathrm{~N}_{2} \mathrm{O}_{3} \mathrm{Na} 283.1053$, found: 283.1064 .

\section{2-(naphthalen-2-yl)-N-phenethylacetamide (4d)}<smiles>O=C(Cc1ccc2ccccc2c1)NCCc1ccccc1</smiles>

4d

Method B, Off-white solid; $20.4 \mathrm{mg}, 71 \%$ yield. ${ }^{1} \mathbf{H}$ NMR $\left(300 \mathrm{MHz}, \mathrm{CDCl}_{3}\right) \delta 7.88-7.72(\mathrm{~m}$, $3 \mathrm{H}), 7.61(\mathrm{~s}, 1 \mathrm{H}), 7.54-7.44(\mathrm{~m}, 2 \mathrm{H}), 7.31-7.23(\mathrm{~m}, 1 \mathrm{H}), 7.13-7.01(\mathrm{~m}, 3 \mathrm{H}), 6.94(\mathrm{dd}, J=7.2$, $2.0 \mathrm{~Hz}, 2 \mathrm{H}), 5.45(\mathrm{~s}, 1 \mathrm{H}), 3.67(\mathrm{~s}, 2 \mathrm{H}), 3.43(\mathrm{q}, J=6.7 \mathrm{~Hz}, 2 \mathrm{H}), 2.68(\mathrm{t}, J=6.8 \mathrm{~Hz}, 2 \mathrm{H}) ;{ }^{13} \mathbf{C} \mathbf{N M R}$ $\left(75 \mathrm{MHz}, \mathrm{CDCl}_{3}\right) \delta 170.8,138.6,133.6,132.5,132.3,128.8,128.6,128.5,128.3,127.7,127.7$, 127.3, 126.5, 126.4, 126.1, 44.1, 40.7, 35.4; HRMS (ESI) m/z: $[\mathrm{M}+\mathrm{Na}]^{+}$calcd for $\mathrm{C}_{20} \mathrm{H}_{19} \mathrm{NONa}$ 312.1359, found: 312.1361 .

\section{N-phenethylcinnamamide (4e)}<smiles>O=C(/C=C/c1ccccc1)NCCc1ccccc1</smiles>

Method C, Off-white solid; $18.3 \mathrm{mg}, 73 \%$ yield. ${ }^{1} \mathbf{H}$ NMR $\left(300 \mathrm{MHz}, \mathrm{CDCl}_{3}\right) \delta 7.62(\mathrm{~d}, J=15.6$ $\mathrm{Hz}, 1 \mathrm{H}), 7.46$ (dd, $J=6.7,3.0 \mathrm{~Hz}, 2 \mathrm{H}), 7.41-7.28(\mathrm{~m}, 5 \mathrm{H}), 7.22(\mathrm{dd}, J=8.9,6.9 \mathrm{~Hz}, 3 \mathrm{H}), 6.36$ $(\mathrm{d}, J=15.6 \mathrm{~Hz}, 1 \mathrm{H}), 5.94(\mathrm{t}, J=5.9 \mathrm{~Hz}, 1 \mathrm{H}), 3.65(\mathrm{q}, J=6.7 \mathrm{~Hz}, 2 \mathrm{H}), 2.88(\mathrm{t}, J=7.0 \mathrm{~Hz}, 2 \mathrm{H})$; 
${ }^{13}$ C NMR $\left(75 \mathrm{MHz}, \mathrm{CDCl}_{3}\right) \delta 166.0,141.0,138.9,134.8,129.7,128.8,128.7,127.8,126.6,120.7$, 40.9, 35.7; HRMS (ESI) m/z: [M+Na $]^{+}$calcd for $\mathrm{C}_{17} \mathrm{H}_{17} \mathrm{NONa} 274.1202$, found: 274.1206.

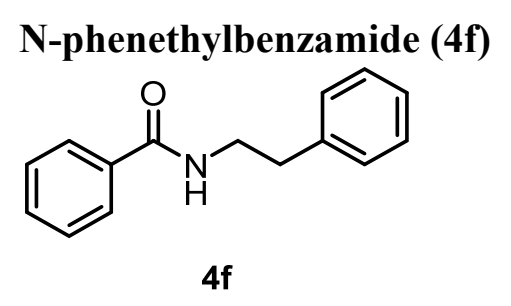

Method C, Off-white solid; $19.7 \mathrm{mg}, 88 \%$ yield. ${ }^{1} \mathbf{H}$ NMR $\left(300 \mathrm{MHz}, \mathrm{CDCl}_{3}\right) \delta 7.74-7.65(\mathrm{~m}$, 2H), $7.48(\mathrm{t}, J=7.3 \mathrm{~Hz}, 1 \mathrm{H}), 7.44-7.29(\mathrm{~m}, 4 \mathrm{H}), 7.25(\mathrm{t}, J=6.3 \mathrm{~Hz}, 3 \mathrm{H}), 6.23(\mathrm{~s}, 1 \mathrm{H}), 3.72$ (q, $J$ $=6.8 \mathrm{~Hz}, 2 \mathrm{H}), 2.93(\mathrm{t}, J=6.9 \mathrm{~Hz}, 2 \mathrm{H}) ;{ }^{13} \mathbf{C} \mathbf{~ N M R}\left(75 \mathrm{MHz}, \mathrm{CDCl}_{3}\right) \delta 167.5,138.9,134.6,131.4$, 128.8, 128.7, 128.6, 126.8, 126.6, 41.2, 35.7; HRMS (ESI) m/z: $[\mathrm{M}+\mathrm{Na}]^{+}$calcd for $\mathrm{C}_{15} \mathrm{H}_{15} \mathrm{NONa}$ 248.1046 , found: 248.1053 .

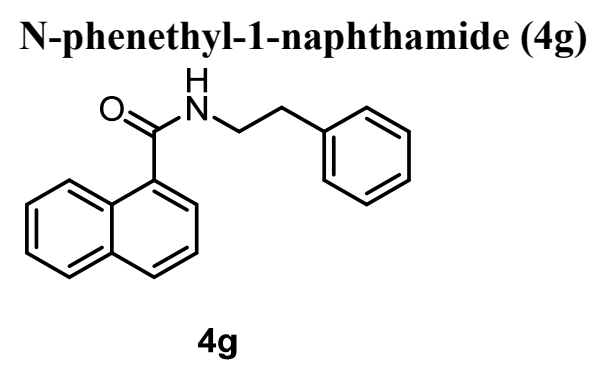

Method C, Off-white solid; $16.5 \mathrm{mg}, 60 \%$ yield. ${ }^{1} \mathbf{H}$ NMR $\left(300 \mathrm{MHz}, \mathrm{CDCl}_{3}\right) \delta 8.26-8.03(\mathrm{~m}$, 1H), $7.93-7.72(\mathrm{~m}, 2 \mathrm{H}), 7.57-7.40(\mathrm{~m}, 3 \mathrm{H}), 7.40-7.12(\mathrm{~m}, 6 \mathrm{H}), 6.16(\mathrm{~s}, 1 \mathrm{H}), 3.74$ (q, $J=6.6$ $\mathrm{Hz}, 2 \mathrm{H}), 2.93(\mathrm{t}, J=6.9 \mathrm{~Hz}, 2 \mathrm{H}) ;{ }^{13} \mathbf{C} \mathbf{N M R}\left(75 \mathrm{MHz}, \mathrm{CDCl}_{3}\right) \delta 169.5,138.7,134.4,133.5,130.4$, 129.9, 128.7, 128.6, 128.1, 126.9, 126.5, 126.3, 125.3, 124.7, 124.6, 41.0, 35.5; HRMS (ESI) m/z: $[\mathrm{M}+\mathrm{Na}]^{+}$calcd for $\mathrm{C}_{19} \mathrm{H}_{17} \mathrm{NONa} 298.1202$, found: 298.1206 .

(9H-fluoren-9-yl)methyl (2-(((1S,4aS,10aR)-7-isopropyl-1,4a-dimethyl-1,2,3,4,4a,9,10,10aoctahydrophenanthren-1-yl)methyl)amino)-2-oxoethyl)carbamate (4h)<smiles>CC(C)c1ccc2c(c1)CC[C@@H]1[C@@H](CNC(=O)CNCF)CCC[C@@]21C</smiles>

4h 
Method B, Off-white solid; $53.2 \mathrm{mg}, 94 \%$ yield. ${ }^{1} \mathbf{H}$ NMR $\left(300 \mathrm{MHz}, \mathrm{CDCl}_{3}\right) \delta 7.73(\mathrm{~d}, J=7.5$ $\mathrm{Hz}, 2 \mathrm{H}), 7.61-7.48(\mathrm{~m}, 2 \mathrm{H}), 7.37$ (t, $J=7.4 \mathrm{~Hz}, 2 \mathrm{H}), 7.31-7.21(\mathrm{~m}, 2 \mathrm{H}), 7.11(\mathrm{~d}, J=8.2 \mathrm{~Hz}$, $1 \mathrm{H}), 6.93(\mathrm{~d}, J=8.0 \mathrm{~Hz}, 1 \mathrm{H}), 6.84(\mathrm{~s}, 1 \mathrm{H}), 6.44(\mathrm{~d}, J=28.4 \mathrm{~Hz}, 1 \mathrm{H}), 5.86(\mathrm{~s}, 1 \mathrm{H}), 4.46-4.23(\mathrm{~m}$, 2H), $4.23-4.06(\mathrm{~m}, 1 \mathrm{H}), 3.82(\mathrm{~d}, J=5.2 \mathrm{~Hz}, 2 \mathrm{H}), 3.14$ (ddd, $J=37.1,13.5,6.4 \mathrm{~Hz}, 2 \mathrm{H}), 2.78$ (dq, $J=20.6,6.7,5.8 \mathrm{~Hz}, 2 \mathrm{H}), 2.22(\mathrm{~d}, J=12.7 \mathrm{~Hz}, 1 \mathrm{H}), 1.90-1.78(\mathrm{~m}, 1 \mathrm{H}), 1.76-1.48(\mathrm{~m}, 4 \mathrm{H})$, $1.36(\mathrm{~d}, J=12.0 \mathrm{~Hz}, 3 \mathrm{H}), 1.16(\mathrm{~d}, J=6.2 \mathrm{~Hz}, 9 \mathrm{H}), 0.89(\mathrm{~s}, 3 \mathrm{H}), 0.81(\mathrm{~s}, 3 \mathrm{H}) ;{ }^{13} \mathbf{C} \mathbf{N M R}(75 \mathrm{MHz}$, $\left.\mathrm{CDCl}_{3}\right) \delta 169.1,156.7,146.9,145.5,143.6,143.5,141.1,134.6,127.6,127.0,127.0,126.8,125.0$, $124.9,124.0,123.8,119.9,67.1,49.9,46.9,45.3,44.6,38.2,37.3,37.3,36.0,33.3,30.1,25.2$, 23.9, 23.8, 19.3, 18.9, 18.5, 18.4; HRMS (ESI) m/z: $[\mathrm{M}+\mathrm{Na}]^{+}$calcd for $\mathrm{C}_{37} \mathrm{H}_{44} \mathrm{~N}_{2} \mathrm{O}_{3} \mathrm{Na}$ 587.3244, found: 587.3248 .

\section{N,N-dibenzyl-2-(naphthalen-2-yl)acetamide (4i)<smiles>O=C(Cc1ccc2ccccc2c1)N(Cc1ccccc1)Cc1ccccc1</smiles> \\ $4 \mathbf{i}$}

Method B, Off-white solid; $36.3 \mathrm{mg}, 99 \%$ yield. ${ }^{1} \mathbf{H}$ NMR $\left(300 \mathrm{MHz}, \mathrm{CDCl}_{3}\right) \delta 7.84-7.76(\mathrm{~m}$, 2H), $7.75-7.68(\mathrm{~m}, 1 \mathrm{H}), 7.64(\mathrm{~s}, 1 \mathrm{H}), 7.42(\mathrm{~m}, 3 \mathrm{H}), 7.38-7.19(\mathrm{~m}, 8 \mathrm{H}), 7.12(\mathrm{~d}, J=6.7 \mathrm{~Hz}, 2 \mathrm{H})$, 4.64 (s, 2H), 4.44 (s, 2H), 3.94 (s, 2H); ${ }^{13} \mathbf{C}$ NMR (75 MHz, $\left.\mathrm{CDCl}_{3}\right) \delta 171.5,137.1,136.3,133.4$, $132.4,132.3,128.9,128.5,128.3,128.2$, 127.6, 127.5, 127.5, 127.3, 127.1, 126.9, 126.3, 126.0, 125.6, 53.4, 50.2, 48.2, 41.1; HRMS (ESI) m/z: [M+Na] $]^{+}$calcd for $\mathrm{C}_{26} \mathrm{H}_{23} \mathrm{NONa} 388.1672$, found: 388.1675 .

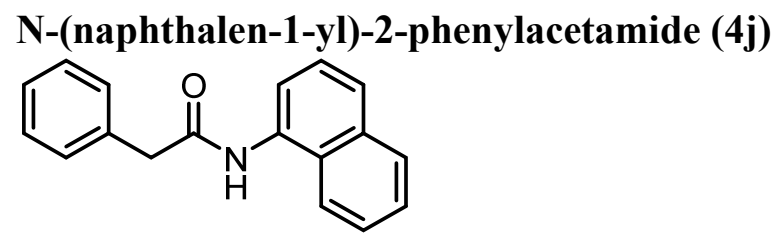

4j

Method C, Off-white solid; $24.5 \mathrm{mg}, 94 \%$ yield. ${ }^{1} \mathbf{H}$ NMR $\left(300 \mathrm{MHz}, \mathrm{CDCl}_{3}\right) \delta 7.95(\mathrm{~d}, J=7.5$ $\mathrm{Hz}, 1 \mathrm{H}), 7.86-7.76(\mathrm{~m}, 1 \mathrm{H}), 7.65(\mathrm{~d}, J=8.2 \mathrm{~Hz}, 1 \mathrm{H}), 7.56-7.34(\mathrm{~m}, 9 \mathrm{H}), 7.29$ (d, $J=8.2 \mathrm{~Hz}$, 1H), $3.88(\mathrm{~s}, 2 \mathrm{H}) ;{ }^{13} \mathbf{C}$ NMR $\left(75 \mathrm{MHz}, \mathrm{CDCl}_{3}\right) \delta 169.6,134.7,134.0,131.9,129.8,129.5,128.8$, 
128.0, 126.8, 126.3, 125.9, 125.7, 120.3, 120.0, 44.9; HRMS (ESI) m/z: $[\mathrm{M}+\mathrm{Na}]^{+}$calcd for $\mathrm{C}_{18} \mathrm{H}_{15} \mathrm{NONa} 284.1046$, found: 284.1046.

\section{N-(4-methoxyphenyl)benzamide (4k)}<smiles>COc1ccc(NC(=O)c2ccccc2)cc1</smiles>

4k

Method C, Off-white solid; $11.1 \mathrm{mg}, 49 \%$ yield. ${ }^{1} \mathbf{H}$ NMR $\left(300 \mathrm{MHz}, \mathrm{CDCl}_{3}\right) \delta 8.12-7.73$ (m, $3 \mathrm{H}), 7.69-7.49(\mathrm{~m}, 3 \mathrm{H}), 7.45(\mathrm{t}, \mathrm{J}=7.4 \mathrm{~Hz}, 2 \mathrm{H}), 6.89(\mathrm{~d}, \mathrm{~J}=8.7 \mathrm{~Hz}, 2 \mathrm{H}), 3.80(\mathrm{~s}, 3 \mathrm{H}) ;{ }^{13} \mathbf{C ~ N M R}$ $\left(75 \mathrm{MHz}_{2} \mathrm{CDCl}_{3}\right) \delta 165.7,156.6,135.0,131.7,131.0,128.7,127.0,122.1,114.2,55.5$; HRMS (ESI) $\mathrm{m} / \mathrm{z}:[\mathrm{M}+\mathrm{Na}]^{+}$calcd for $\mathrm{C}_{14} \mathrm{H}_{13} \mathrm{NO}_{2} \mathrm{Na}_{2} 250.0838$, found: 250.0838 .

2-(naphthalen-2-yl)-N-phenylacetamide (4I)<smiles>O=C(Cc1ccc2ccccc2c1)Nc1ccccc1</smiles>

41

Method C, Off-white solid; $26.5 \mathrm{mg},>99 \%$ yield. ${ }^{1} \mathbf{H}$ NMR $\left(300 \mathrm{MHz}, \mathrm{CDCl}_{3}\right) \delta 8.03-7.66(\mathrm{~m}$, 4H), $7.63-7.33(\mathrm{~m}, 6 \mathrm{H}), 7.24(\mathrm{t}, J=7.9 \mathrm{~Hz}, 2 \mathrm{H}), 7.06(\mathrm{t}, J=7.4 \mathrm{~Hz}, 1 \mathrm{H}), 3.84(\mathrm{~s}, 2 \mathrm{H}) ;{ }^{13} \mathbf{C} \mathbf{N M R}$ $\left(75 \mathrm{MHz}, \mathrm{CDCl}_{3}\right) \delta 169.3,137.7,133.6,132.6,131.9,129.0,129.0,128.4,127.8,127.7,127.3$, 126.6, 126.2 , 124.5, 119.9, 44.9; HRMS (ESI) m/z: $[\mathrm{M}+\mathrm{H}]^{+}$calcd for $\mathrm{C}_{18} \mathrm{H}_{16} \mathrm{NO} 262.1226$, found: 262.1220 .

\section{5-nitro-6-thiocyanatopyrimidin-4-ol}<smiles>N#Cc1ncnc(O)c1[N+](=O)[O-]</smiles>

${ }^{1} \mathbf{H}$ NMR (300 MHz, DMSO-d6) $\delta 9.76(\mathrm{~s}, 1 \mathrm{H}), 8.18(\mathrm{~s}, 1 \mathrm{H}) ;{ }^{13} \mathbf{C}$ NMR $\left(75 \mathrm{MHz}, \mathrm{DMSO}-d_{6}\right) \delta$ 44.0; HRMS (ESI) m/z: [M+Na] $]^{+}$calcd for $\mathrm{C}_{5} \mathrm{H}_{2} \mathrm{~N}_{4} \mathrm{O}_{3} \mathrm{SNa} 220.9740$, found: 220.9730 .

\section{Benzoic Cyanic Thioanhydride}


<smiles>N#[As]C(=O)c1ccccc1</smiles>

${ }^{1} \mathbf{H}$ NMR $\left(300 \mathrm{MHz}, \mathrm{CDCl}_{3}\right) \delta 8.05(\mathrm{dd}, J=8.0,1.5 \mathrm{~Hz}, 2 \mathrm{H}), 7.74-7.60(\mathrm{~m}, 1 \mathrm{H}), 7.50(\mathrm{t}, J=7.7$ $\mathrm{Hz}, 2 \mathrm{H}) ;{ }^{13} \mathbf{C}$ NMR $\left(75 \mathrm{MHz}, \mathrm{CDCl}_{3}\right) \delta 161.8,135.1,130.8,130.5,129.0$; HRMS (ESI) m/z: $[\mathrm{M}+\mathrm{Na}]^{+}$calcd for $\mathrm{C}_{8} \mathrm{H}_{5} \mathrm{NOSNa} 185.9984$, found: 185.9992 . 


\section{HPLC Spectra for Determining Epimerization of 3aa}

Chiralpak ${ }^{\circledR} \mathrm{IC}$ column, hexane/EtOH (8:2), flow rate $1.0 \mathrm{~mL} / \mathrm{min}$

HPLC Spectra of Fmoc-Gly-D/L-Ala-OMe

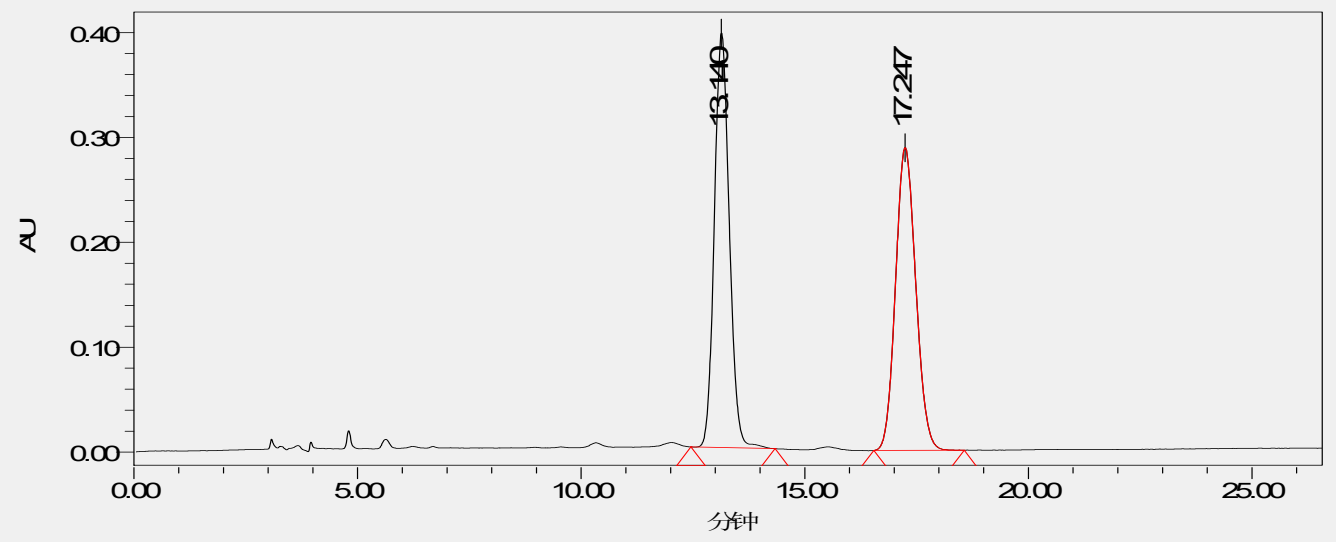

\begin{tabular}{|c|c|c|c|c|c|}
\hline Peak No & Retention time & Area \% & Area \% Area & Height & Integral type \\
\hline 1 & 13.140 & 9189955 & 50.29 & 395146 & $\mathrm{bb}$ \\
\hline 2 & 17.247 & 9082724 & 49.71 & 288589 & $\mathrm{bb}$ \\
\hline
\end{tabular}

HPLC Spectra of 3aa

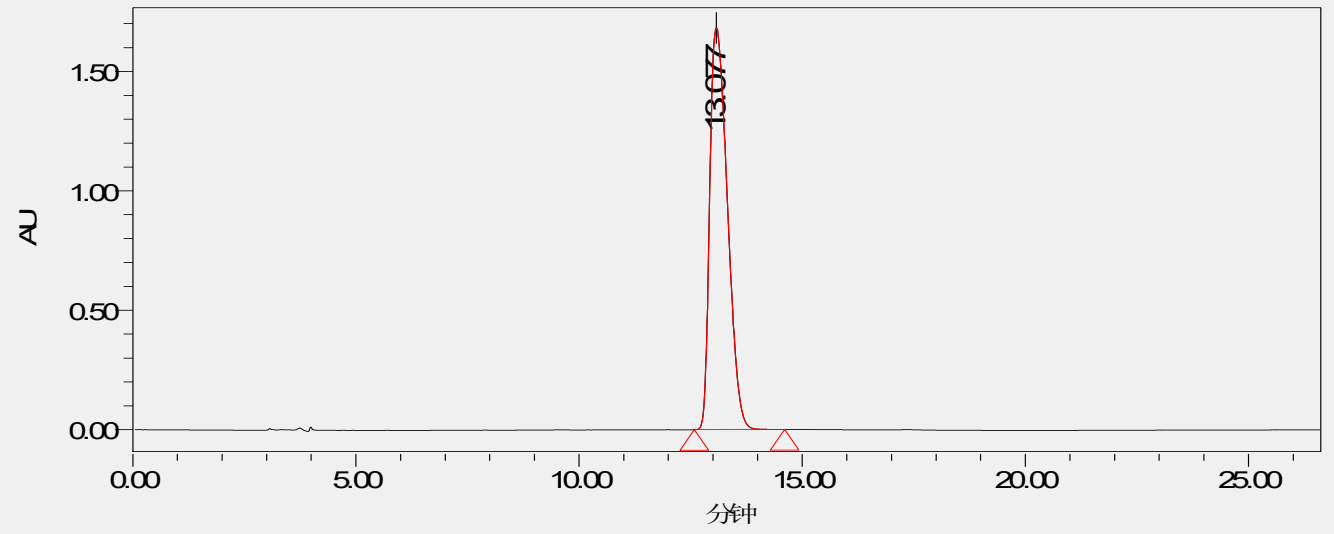

\begin{tabular}{|c|c|c|c|c|c|}
\hline Peak No & Retention time & Area \% & Area \% Area & Height & Integral type \\
\hline 1 & 13.077 & 46320448 & 100.00 & 1682069 & bb \\
\hline
\end{tabular}




\section{HPLC Spectra for Determining Epimerization of 3ab}

Chiralpak ${ }^{\circledR} \mathrm{IC}$ column, hexane/EtOH (8:2), flow rate $1.0 \mathrm{~mL} / \mathrm{min}$

HPLC Spectra of Mixed Fmoc-Gly-D/L-Leu-OMe

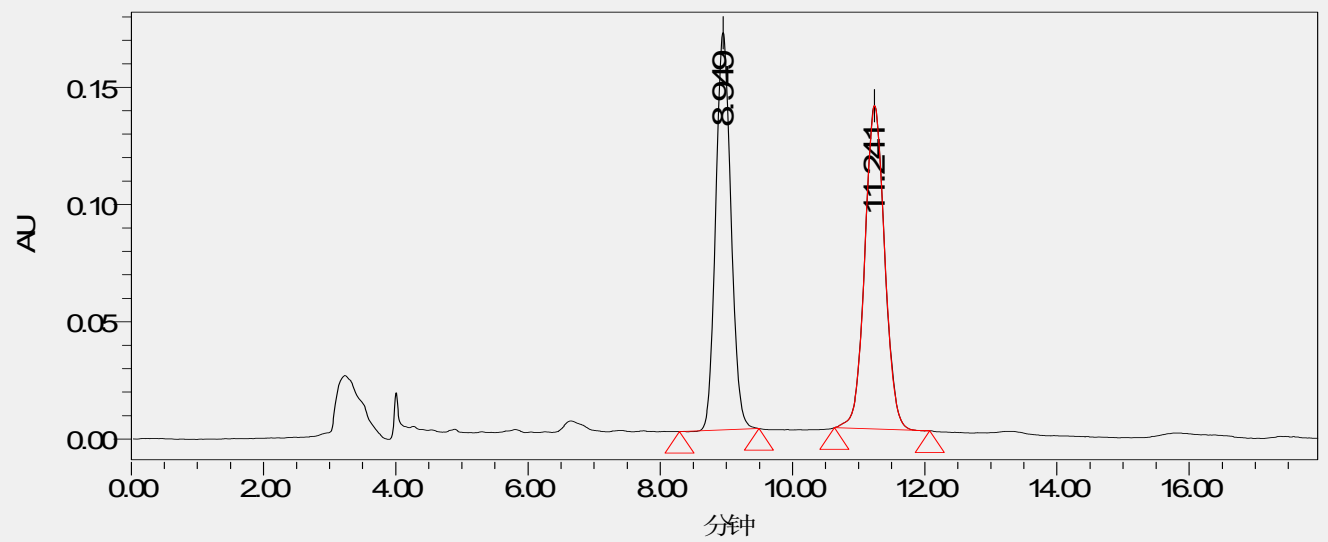

\begin{tabular}{|c|c|c|c|c|c|}
\hline Peak No & Retention time & Area \% & Area \% Area & Height & Integral type \\
\hline 1 & 8.949 & 2713867 & 48.43 & 169353 & $\mathrm{bb}$ \\
\hline 2 & 11.241 & 2889759 & 51.57 & 137864 & $\mathrm{bb}$ \\
\hline
\end{tabular}

HPLC Spectra of $\mathbf{3 a b}$

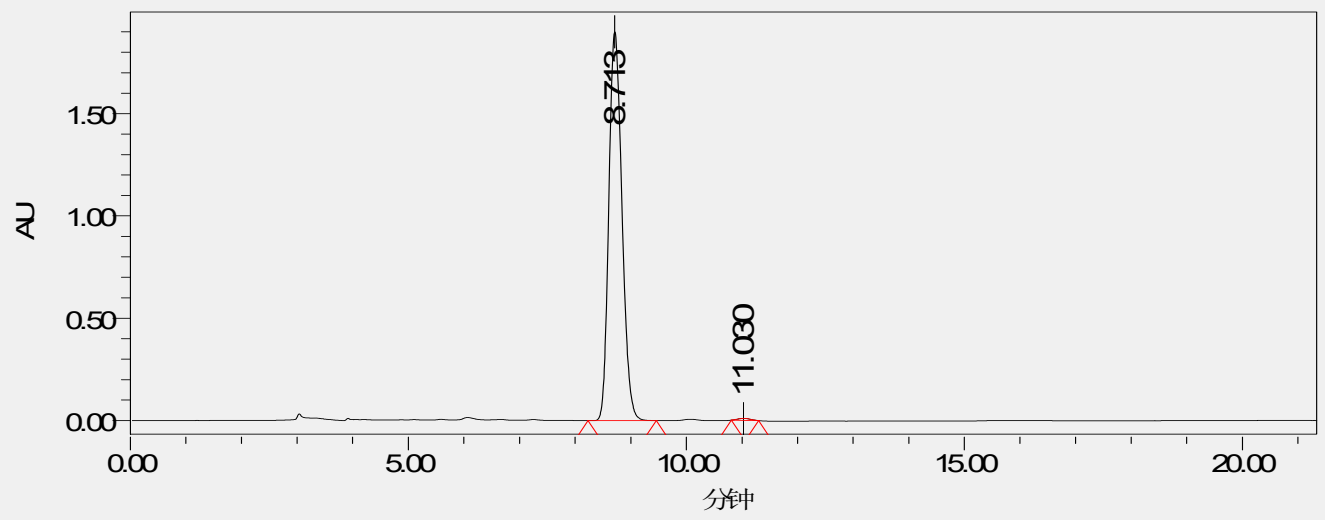

\begin{tabular}{|c|c|c|c|c|c|}
\hline Peak No & Retention time & Area \% & Area \% Area & Height & Integral type \\
\hline 1 & 8.713 & 31096671 & 99.52 & 1902052 & $\mathrm{bb}$ \\
\hline 2 & 11.030 & 149485 & 0.48 & 8879 & $\mathrm{bb}$ \\
\hline
\end{tabular}




\section{HPLC Spectra for Determining Epimerization of 3ad}

Chiralpak ${ }^{\circledR} \mathrm{IC}$ column, hexane/EtOH (8:2), flow rate $1.0 \mathrm{~mL} / \mathrm{min}$

HPLC Spectra of Mixed Fmoc-Gly-D/L-Phe-OMe

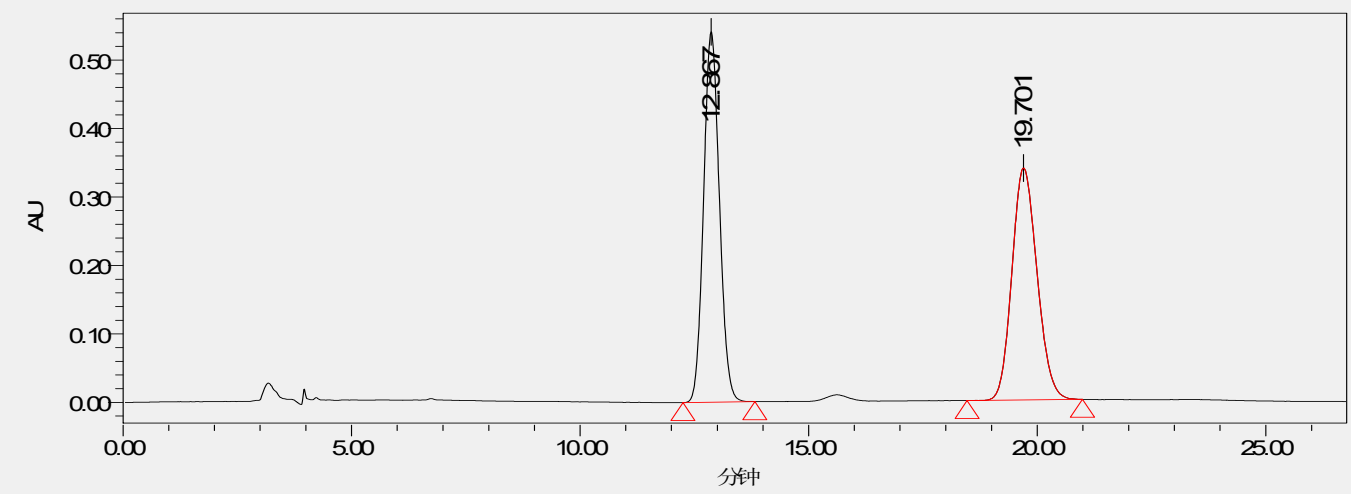

\begin{tabular}{|c|c|c|c|c|c|}
\hline Peak No & Retention time & Area \% & Area \% Area & Height & Integral type \\
\hline 1 & 12.867 & 13023885 & 50.14 & 540922 & $\mathrm{bb}$ \\
\hline 2 & 19.701 & 12949700 & 49.86 & 338850 & $\mathrm{bb}$ \\
\hline
\end{tabular}

HPLC Spectra of 3ad

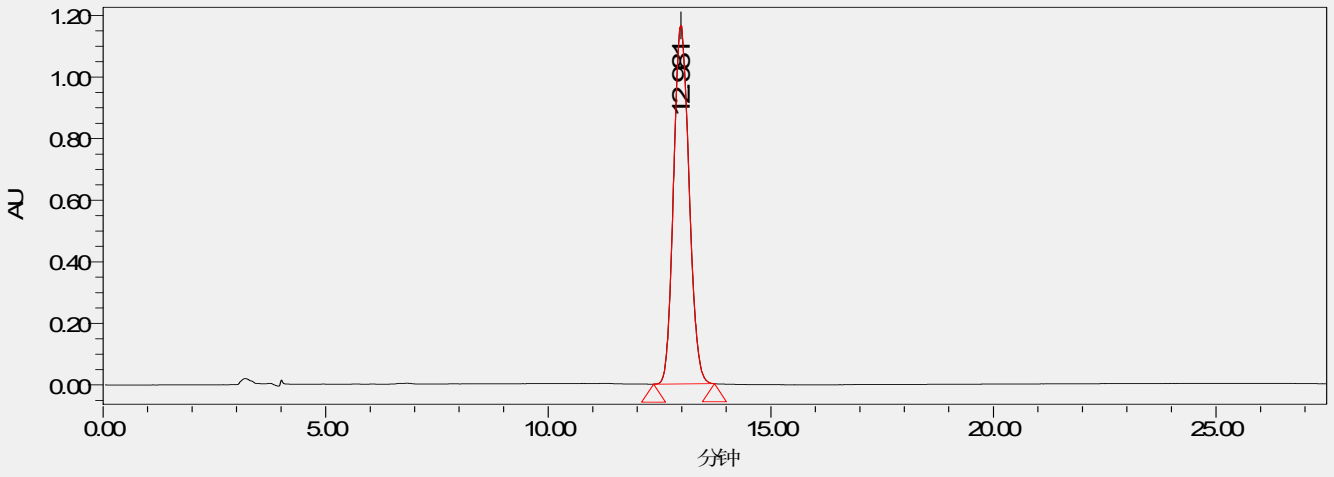

\begin{tabular}{|c|c|c|c|c|c|}
\hline Peak No & Retention time & Area \% & Area \% Area & Height & Integral type \\
\hline 1 & 12.981 & 28291232 & 100.00 & 1164576 & $\mathrm{bb}$ \\
\hline
\end{tabular}




\section{HPLC Spectra for Determining Epimerization of 3ae}

Chiralpak ${ }^{\circledR} \mathrm{IC}$ column, hexane/EtOH (8:2), flow rate $1.0 \mathrm{~mL} / \mathrm{min}$

HPLC Spectra of Mixed Fmoc-Gly-D/L-Trp-OMe

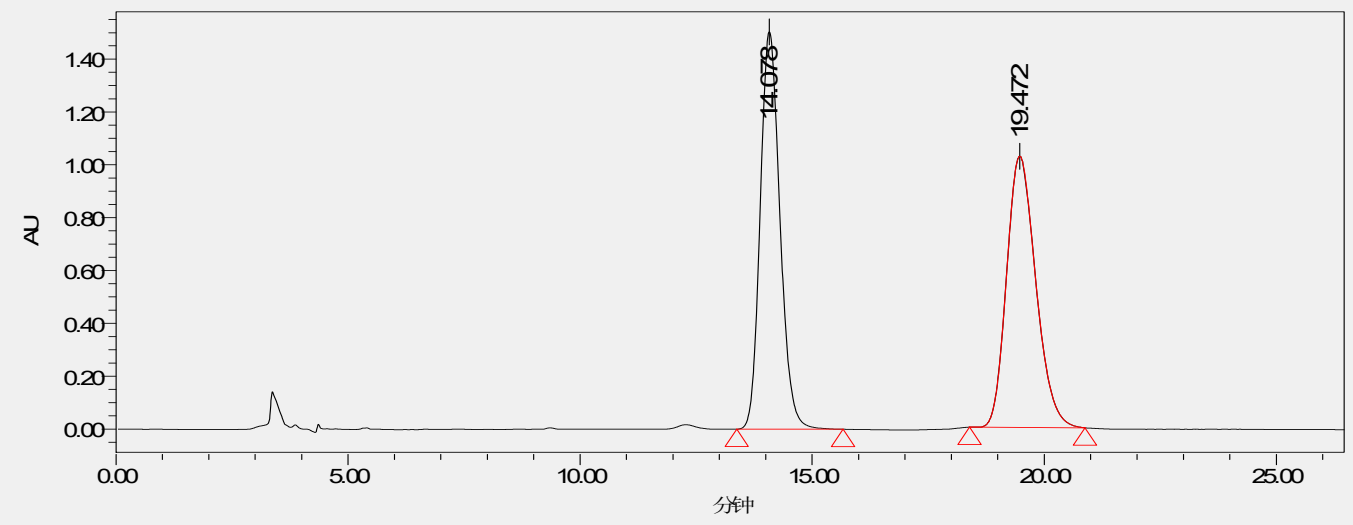

\begin{tabular}{|c|c|c|c|c|c|}
\hline Peak No & Retention time & Area \% & Area \% Area & Height & Integral type \\
\hline 1 & 14.078 & 44550098 & 49.77 & 1504060 & $\mathrm{bb}$ \\
\hline 2 & 19.472 & 44967169 & 50.23 & 1026542 & $\mathrm{bb}$ \\
\hline
\end{tabular}

HPLC Spectra of $\mathbf{3 a e}$

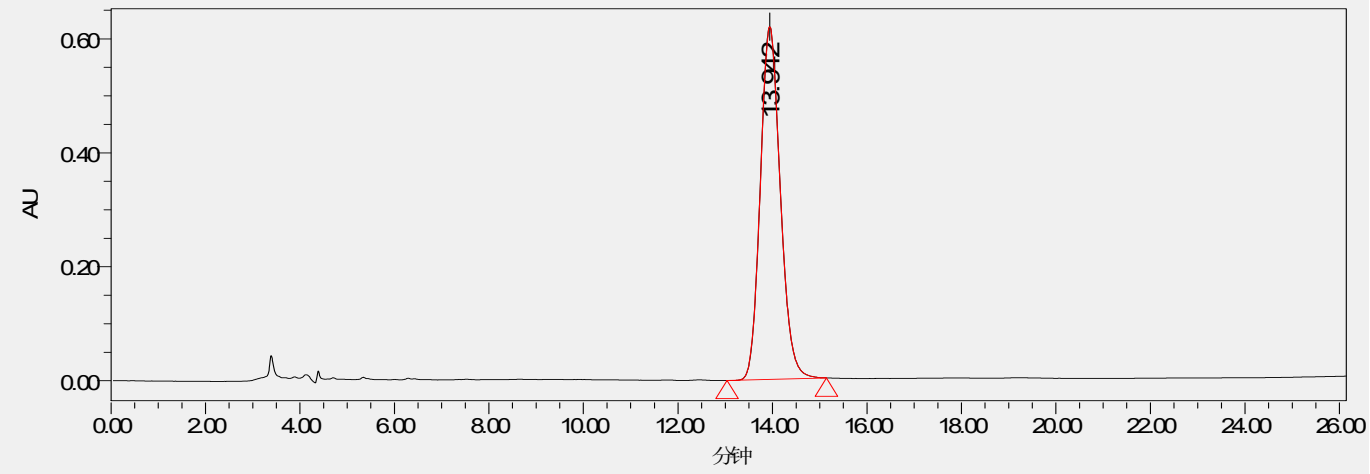

\begin{tabular}{|c|c|c|c|c|c|}
\hline Peak No & Retention time & Area \% & Area \% Area & Height & Integral type \\
\hline 1 & 13.942 & 18218250 & 100.00 & 618827 & bb \\
\hline
\end{tabular}




\section{HPLC Spectra for Determining Epimerization of 3af}

Chiralpak ${ }^{\circledR} \mathrm{IC}$ column, hexane/EtOH (8:2), flow rate $1.0 \mathrm{~mL} / \mathrm{min}$

HPLC Spectra of Mixed Fmoc-Gly-D/L-Tyr-OMe

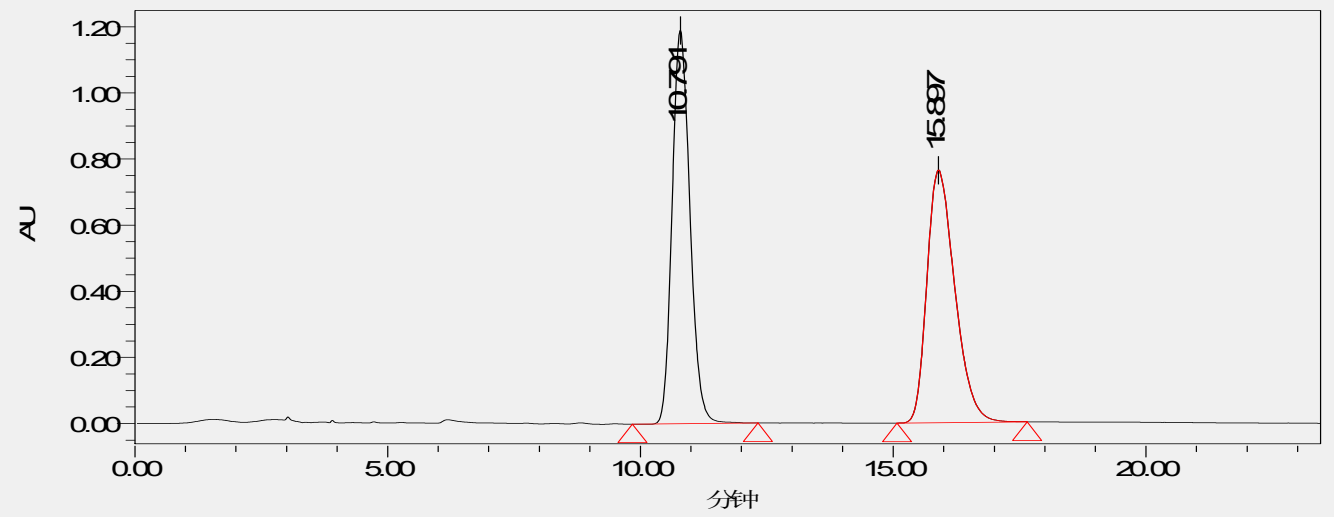

\begin{tabular}{|c|c|c|c|c|c|}
\hline Peak No & Retention time & Area \% & Area \% Area & Height & Integral type \\
\hline 1 & 10.791 & 29206555 & 49.87 & 1189386 & $\mathrm{bb}$ \\
\hline 2 & 15.897 & 29360418 & 50.13 & 763836 & $\mathrm{bb}$ \\
\hline
\end{tabular}

HPLC Spectra of $\mathbf{3 a f}$

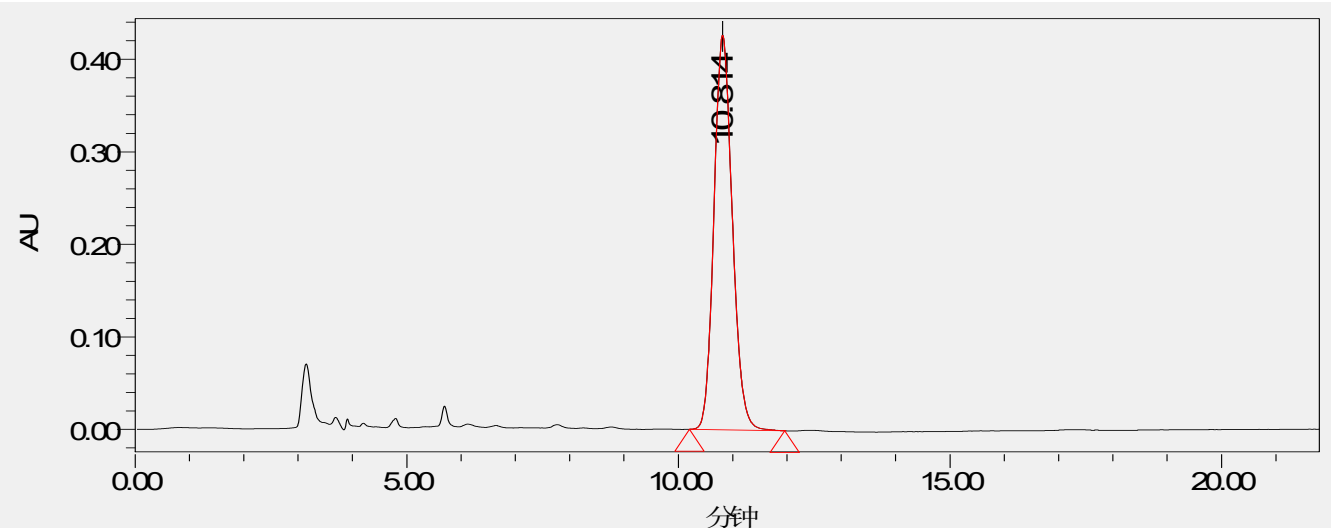

\begin{tabular}{|c|c|c|c|c|c|}
\hline Peak No & Retention time & Area \% & Area \% Area & Height & Integral type \\
\hline 1 & 10.814 & 10037154 & 100.00 & 426373 & $\mathrm{bb}$ \\
\hline
\end{tabular}




\section{HPLC Spectra for Determining Epimerization of 3ag}

Chiralpak ${ }^{\circledR} \mathrm{IC}$ column, hexane/EtOH (8:2), flow rate $1.0 \mathrm{~mL} / \mathrm{min}$

HPLC Spectra of Mixed Fmoc-Gly-D/L-Ser-OMe

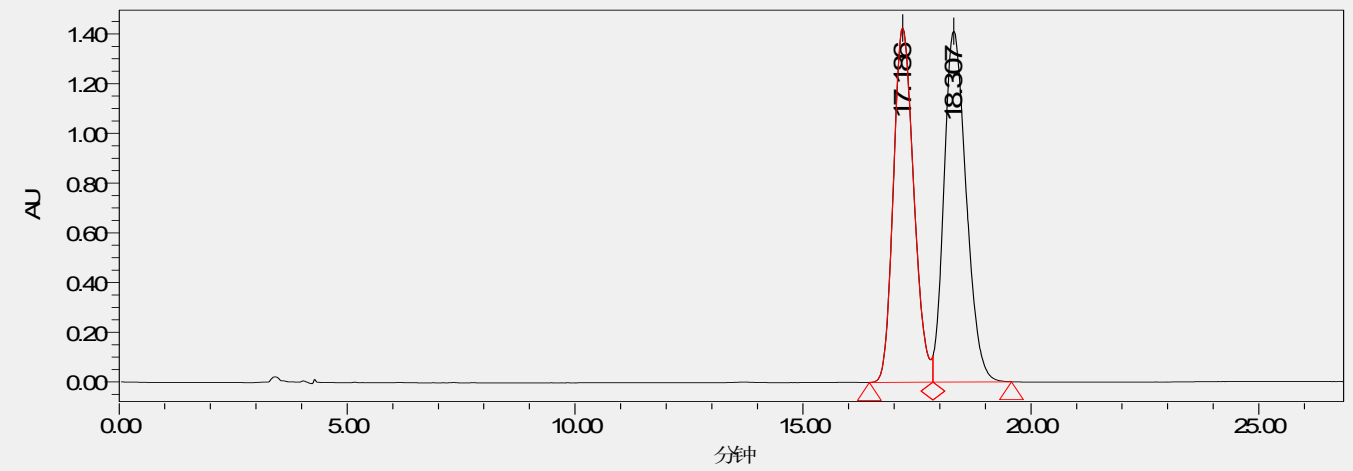

\begin{tabular}{|c|c|c|c|c|c|}
\hline Peak No & Retention time & Area \% & Area \% Area & Height & Integral type \\
\hline 1 & 17.186 & 44405172 & 48.12 & 1425140 & bv \\
\hline 2 & 18.307 & 47866661 & 51.88 & 1411023 & $\mathrm{vb}$ \\
\hline
\end{tabular}

HPLC Spectra of $\mathbf{3 a g}$

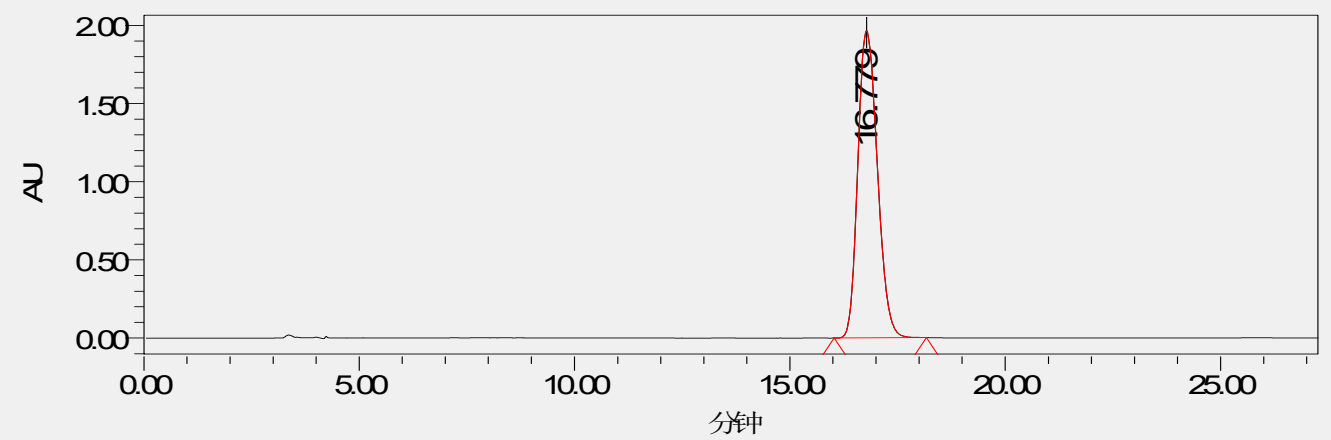

\begin{tabular}{|c|c|c|c|c|c|}
\hline Peak No & Retention time & Area \% & Area \% Area & Height & Integral type \\
\hline 1 & 16.779 & 61993645 & 100.00 & 1965103 & $\mathrm{bb}$ \\
\hline
\end{tabular}




\section{HPLC Spectra for Determining Epimerization of 3ah}

Chiralpak ${ }^{\circledR} \mathrm{IC}$ column, hexane/EtOH (8:2), flow rate $1.0 \mathrm{~mL} / \mathrm{min}$

HPLC Spectra of Mixed Fmoc-Gly-D/L-Lys(Boc)-OMe

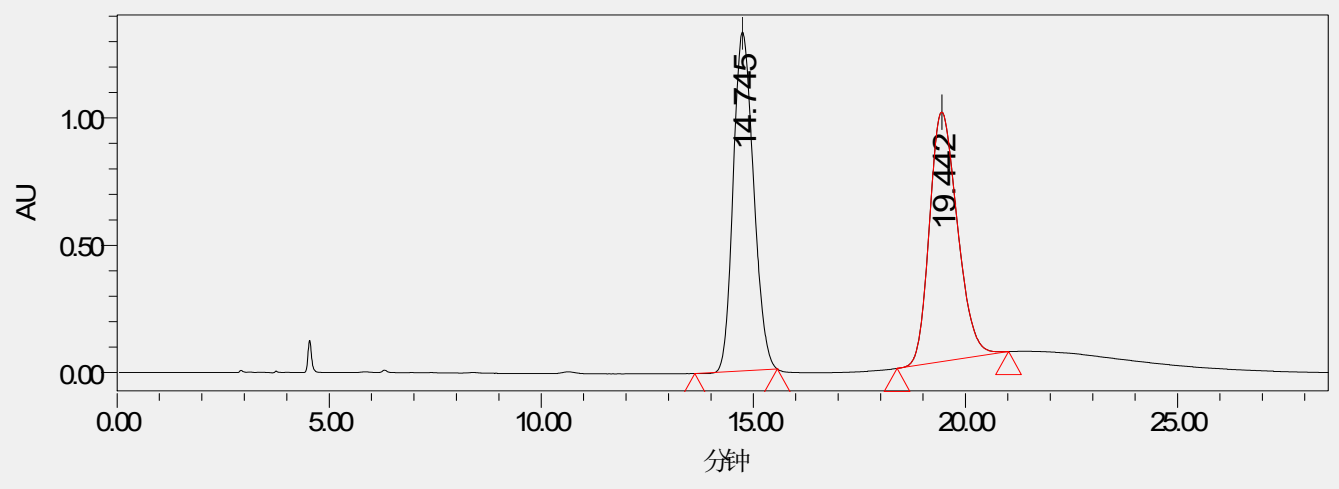

\begin{tabular}{|c|c|c|c|c|c|}
\hline Peak No & Retention time & Area \% & Area \% Area & Height & Integral type \\
\hline 1 & 14.745 & 44385180 & 49.75 & 1331020 & $\mathrm{bb}$ \\
\hline 2 & 19.442 & 44828895 & 50.25 & 979711 & $\mathrm{bb}$ \\
\hline
\end{tabular}

HPLC Spectra of $\mathbf{3 a h}$

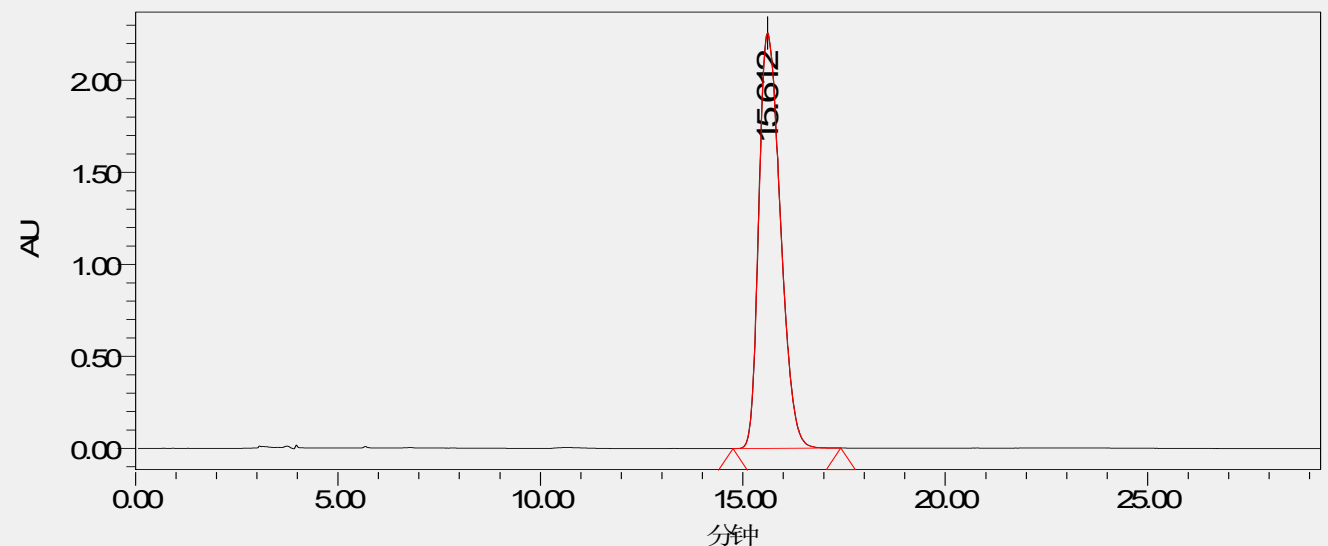

\begin{tabular}{|c|c|c|c|c|c|}
\hline Peak No & Retention time & Area \% & Area \% Area & Height & Integral type \\
\hline 1 & 15.612 & 83716030 & 100.00 & 2257933 & bb \\
\hline
\end{tabular}




\section{Scale-up of the Reaction}

NDTP was used in the synthesis of dipeptides on the gram scale.

Method: A $100 \mathrm{~mL}$ round-bottom flask equipped with a stir bar was charged with carboxylic acid (4.5 mmol), amine hydrochloride (3.0 mmol), DIPEA (12.0 mmol) and DCM (20 mL), the solution of NDTP $(4.5 \mathrm{mmol})$ in DCM $(10 \mathrm{~mL})$ was added and stirred for 1 minute at room temperature. Then the solvent was evaporated under reduced pressure, and the residue was purified by flash column chromatography $\left(\mathrm{EtOAc} /\right.$ hexane $=1: 1-1: 3$ or $\left.\mathrm{MeOH} / \mathrm{CH}_{2} \mathrm{Cl}_{2}=1: 40\right)$. Especially, the solvent was $\mathrm{MeCN}$ in the synthesis of 3ea.

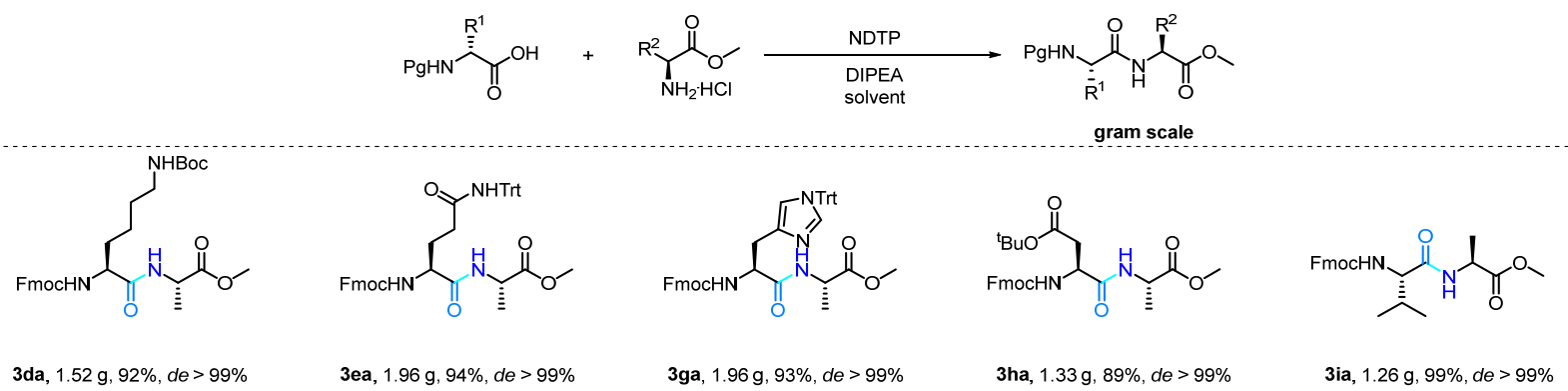


HPLC Spectra for Determining Epimerization of 3da

Chiralpak ${ }^{\circledR} \mathrm{IA}$ column, hexane/iPrOH (8:2), flow rate $1.0 \mathrm{~mL} / \mathrm{min}$ HPLC Spectra of Mixed Fmoc-L-Lys(Boc)-L-Ala-OMe (3da) and Fmoc-D-Lys(Boc)-L-Ala-OMe

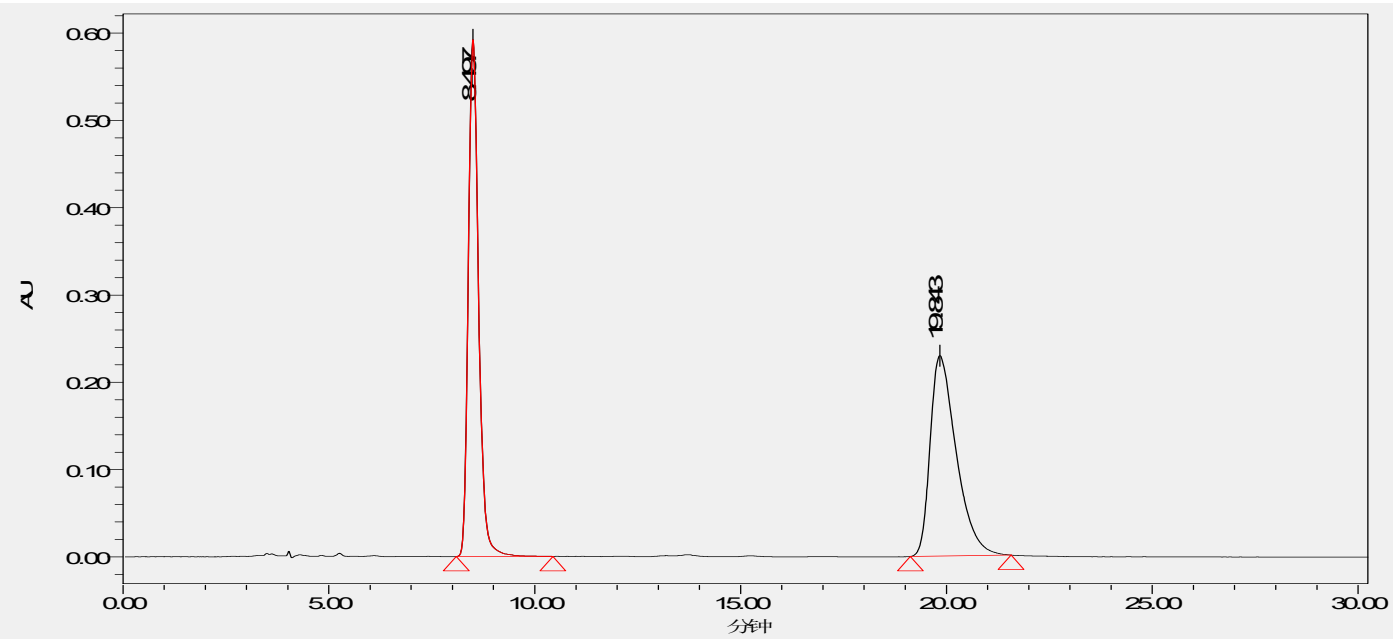

\begin{tabular}{|c|c|c|c|c|c|}
\hline Peak No & Retention time & Area \% & Area \% Area & Height & Integral type \\
\hline 1 & 8.497 & 10283001 & 50.39 & 592120 & bb \\
\hline 2 & 19.843 & 10122208 & 49.61 & 229579 & bb \\
\hline
\end{tabular}

HPLC Spectra of 3da

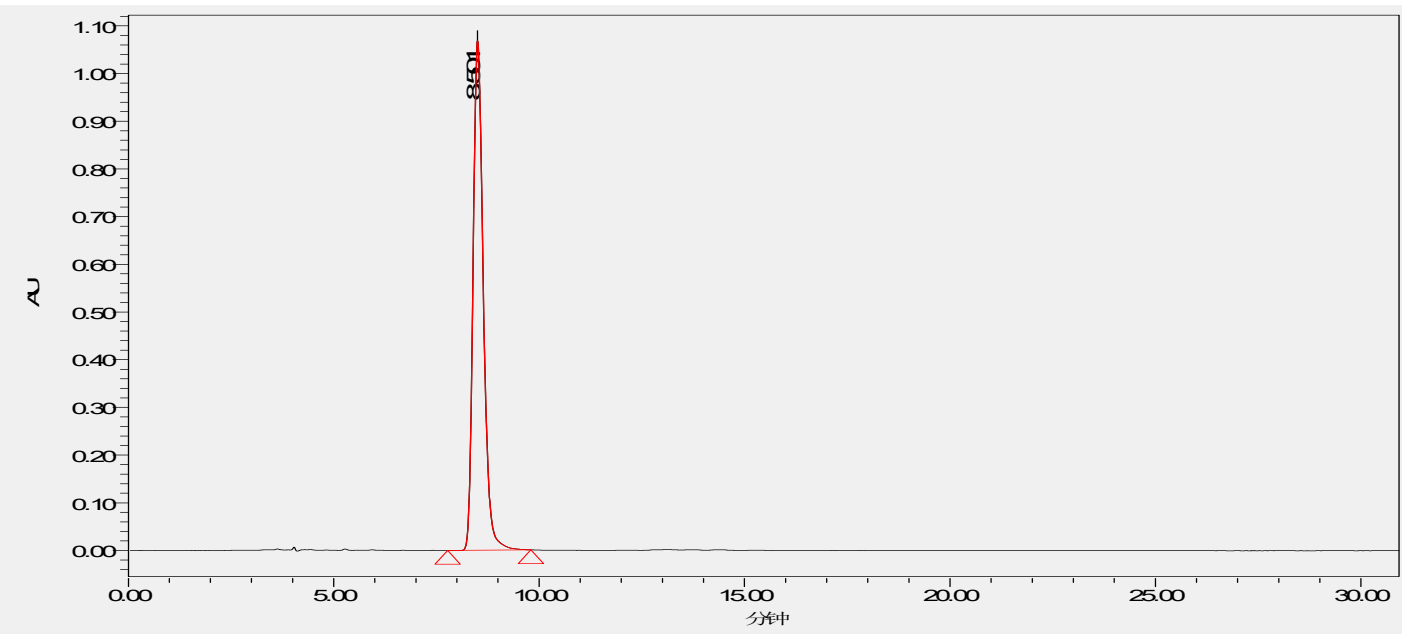

Peak Info

\begin{tabular}{|c|c|c|c|c|c|}
\hline Peak No & Retention time & Area \% & Area \% Area & Height & Integral type \\
\hline 1 & 8.501 & 18546196 & 100.00 & 1068508 & bb \\
\hline
\end{tabular}




\section{HPLC Spectra for Determining Epimerization of 3ea}

Chiralpak ${ }^{\circledR} \mathrm{IA}$ column, hexane/iPrOH (9:1), flow rate $1.0 \mathrm{~mL} / \mathrm{min}$ HPLC Spectra of Mixed Fmoc-L-Gln(Trt)-L-Ala-OMe (3ea) and Fmoc-D-Gln(Trt)-L-Ala-OMe

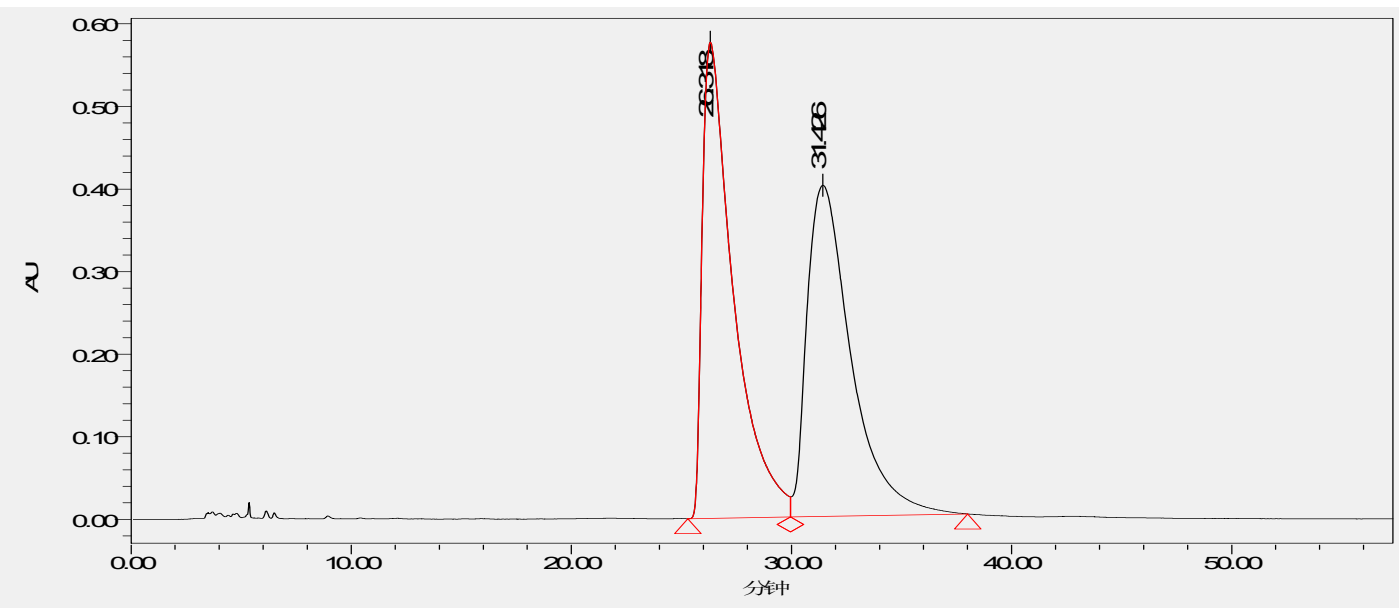

\begin{tabular}{|c|c|c|c|c|c|}
\hline Peak No & Retention time & Area \% & Area \% Area & Height & Integral type \\
\hline 1 & 26.318 & 56852115 & 50.43 & 576571 & bv \\
\hline 2 & 31.426 & 55887446 & 49.57 & 401063 & vb \\
\hline
\end{tabular}

HPLC data of 3ea

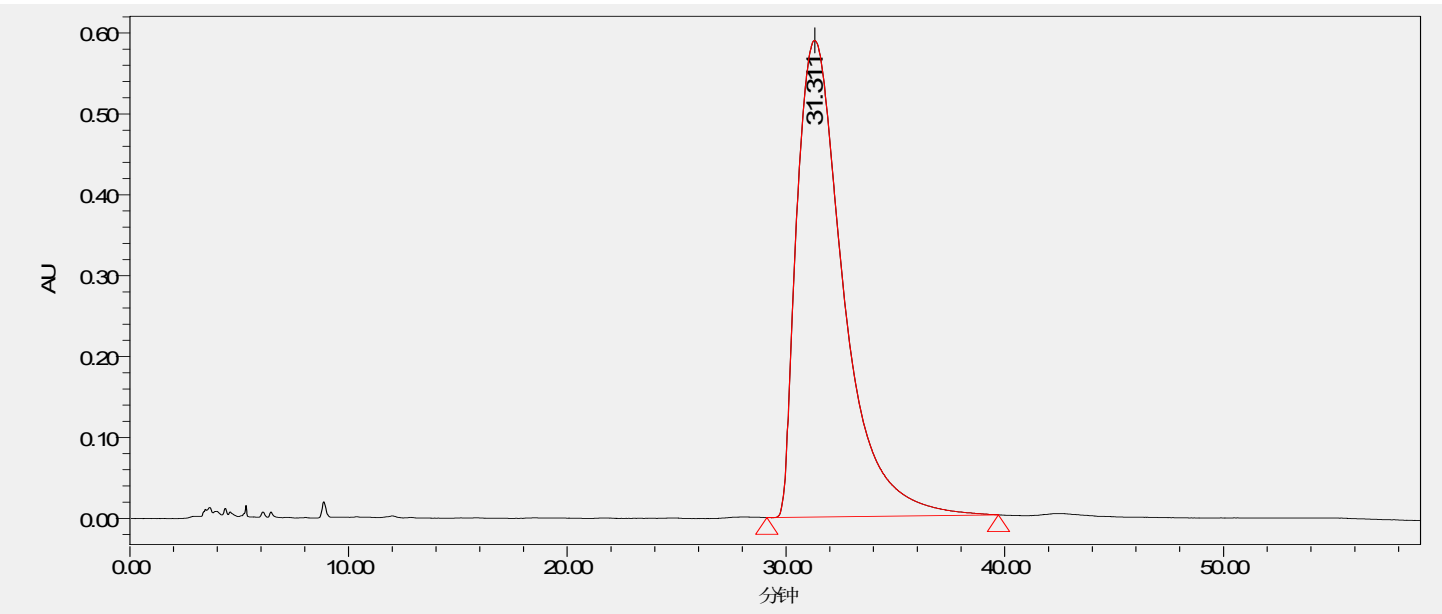

\begin{tabular}{|c|c|c|c|c|c|}
\hline Peak No & Retention time & Area \% & Area \% Area & Height & Integral type \\
\hline 1 & 31.311 & 87636307 & 100.00 & 589168 & bb \\
\hline
\end{tabular}




\section{HPLC Spectra for Determining Epimerization of 3ga}

Chiralpak ${ }^{\circledR} \mathrm{IA}$ column, hexane/iPrOH (8:2), flow rate $1.0 \mathrm{~mL} / \mathrm{min}$ HPLC Spectra of Mixed Fmoc-L-His(Trt)-L-Ala-OMe (3ga) and Fmoc-D-His(Trt)-L-Ala-OMe

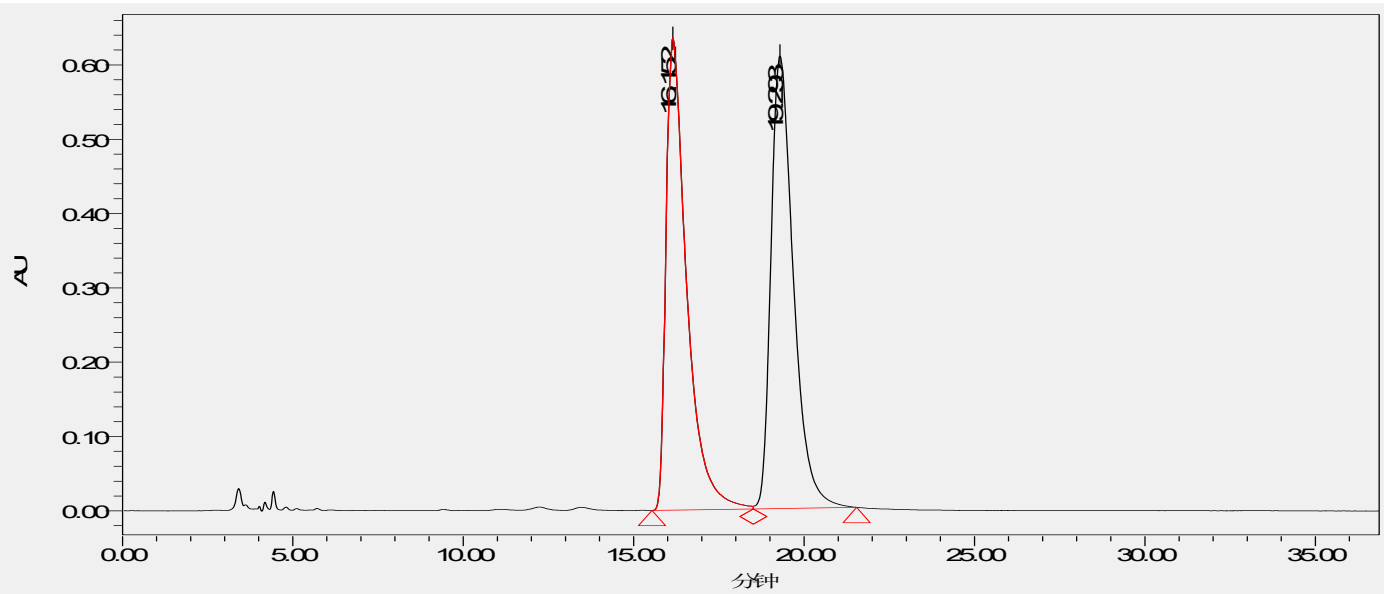

\begin{tabular}{|c|c|c|c|c|c|}
\hline Peak No & Retention time & Area \% & Area \% Area & Height & Integral type \\
\hline 1 & 16.152 & 26862249 & 49.03 & 634959 & bv \\
\hline 2 & 19.293 & 27923572 & 50.97 & 608930 & vb \\
\hline
\end{tabular}

HPLC Spectra of 3ga

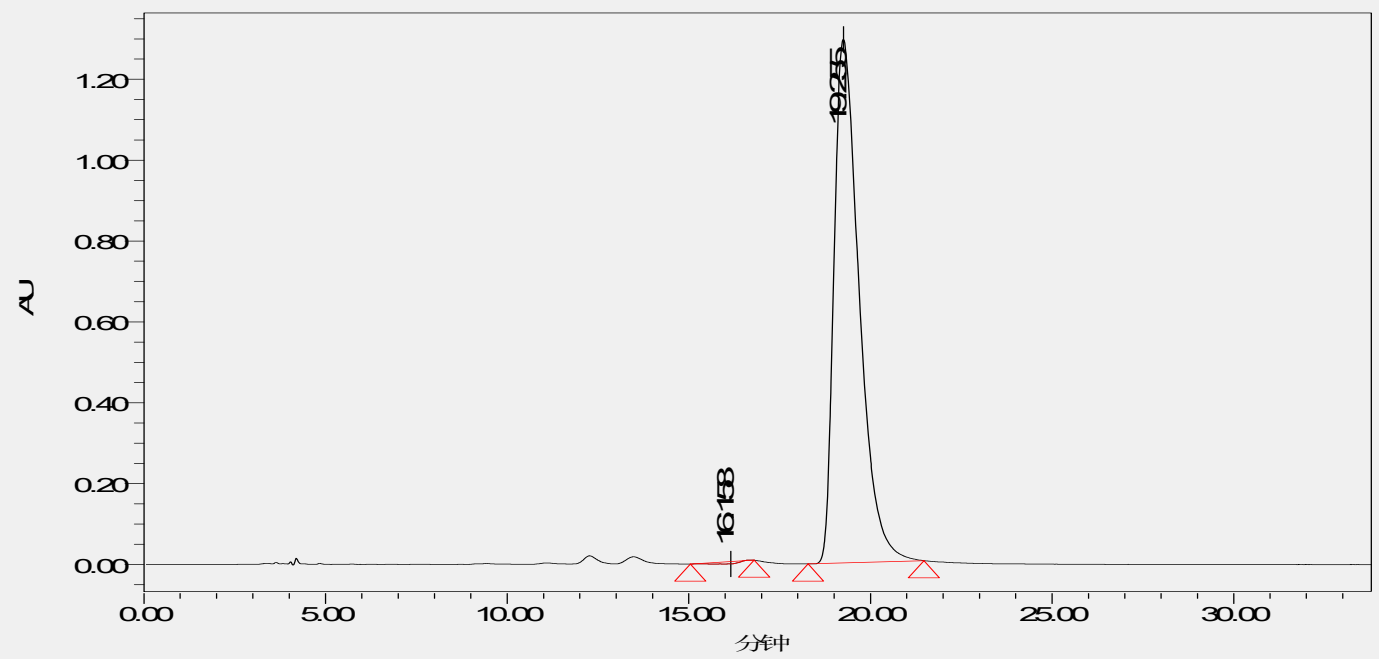

\begin{tabular}{|c|c|c|c|c|c|}
\hline Peak No & Retention time & Area \% & Area \% Area & Height & Integral type \\
\hline 1 & 16.158 & 287489 & 0.46 & -5768 & bb \\
\hline 2 & 19.255 & 62325111 & 99.54 & 1294768 & bb \\
\hline
\end{tabular}




\section{HPLC Spectra for Determining Epimerization of 3ha}

Chiralpak ${ }^{\circledR}$ IA column, hexane/iPrOH (8:2), flow rate $1.0 \mathrm{~mL} / \mathrm{min}$ HPLC Spectra of Mixed Fmoc-L-Asp(tBu)-L-Ala-OMe (3ha) and Fmoc-D-Asp(tBu)-L-Ala-OMe

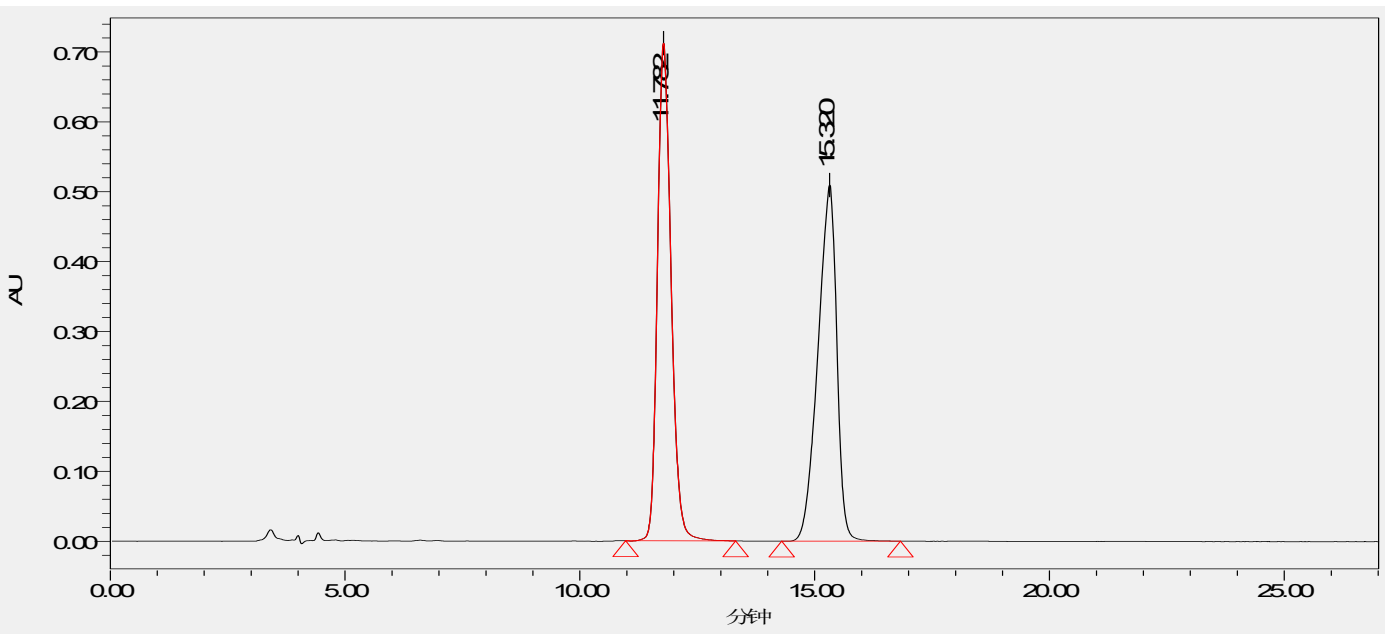

\begin{tabular}{|c|c|c|c|c|c|}
\hline Peak No & Retention time & Area \% & Area \% Area & Height & Integral type \\
\hline 1 & 11.782 & 14428398 & 50.34 & 712138 & bb \\
\hline 2 & 15.320 & 14236040 & 49.66 & 509652 & bb \\
\hline
\end{tabular}

HPLC data of 3ha

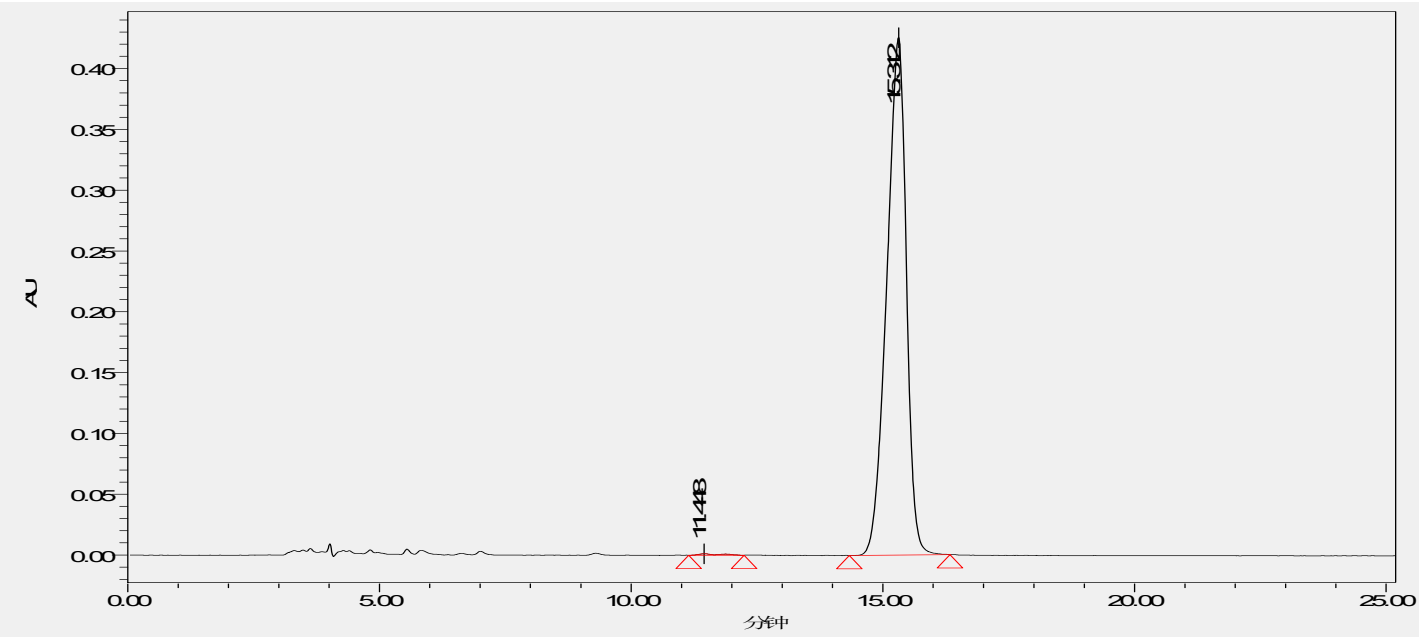

\begin{tabular}{|c|c|c|c|c|c|}
\hline Peak No & Retention time & Area \% & Area \% Area & Height & Integral type \\
\hline 1 & 11.448 & 35652 & 0.31 & 1100 & $\mathrm{bb}$ \\
\hline 2 & 15.312 & 11471175 & 99.69 & 425512 & $\mathrm{bb}$ \\
\hline
\end{tabular}




\section{HPLC Spectra for Determining Epimerization of 3ia}

Chiralpak ${ }^{\circledR} \mathrm{IC}$ column, hexane/iPrOH (8:2), flow rate $1.0 \mathrm{~mL} / \mathrm{min}$

HPLC Spectra of Mixed Fmoc-L-Val-L-Ala-OMe (3ia) and Fmoc-D-Val-L-Ala-OMe

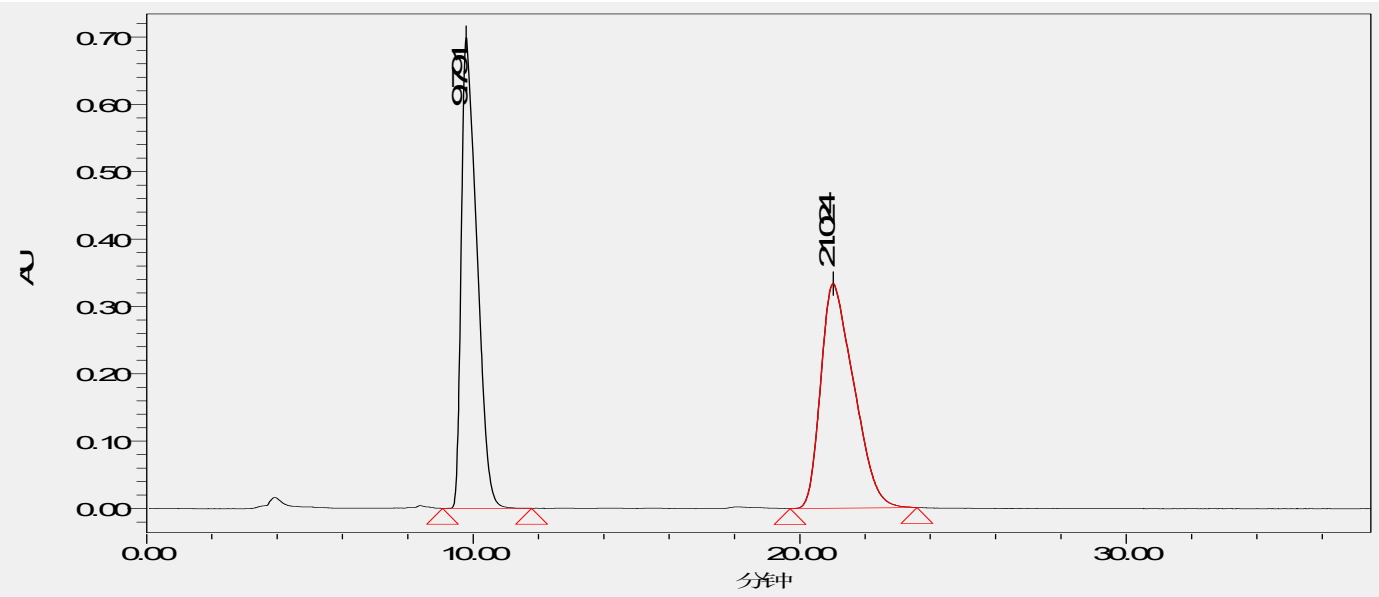

\begin{tabular}{|c|c|c|c|c|c|}
\hline Peak No & Retention time & Area \% & Area \% Area & Height & Integral type \\
\hline 1 & 9.791 & 23147102 & 49.69 & 698778 & bb \\
\hline 2 & 21.024 & 23439713 & 50.31 & 333370 & bb \\
\hline
\end{tabular}

HPLC Spectra of 3ia

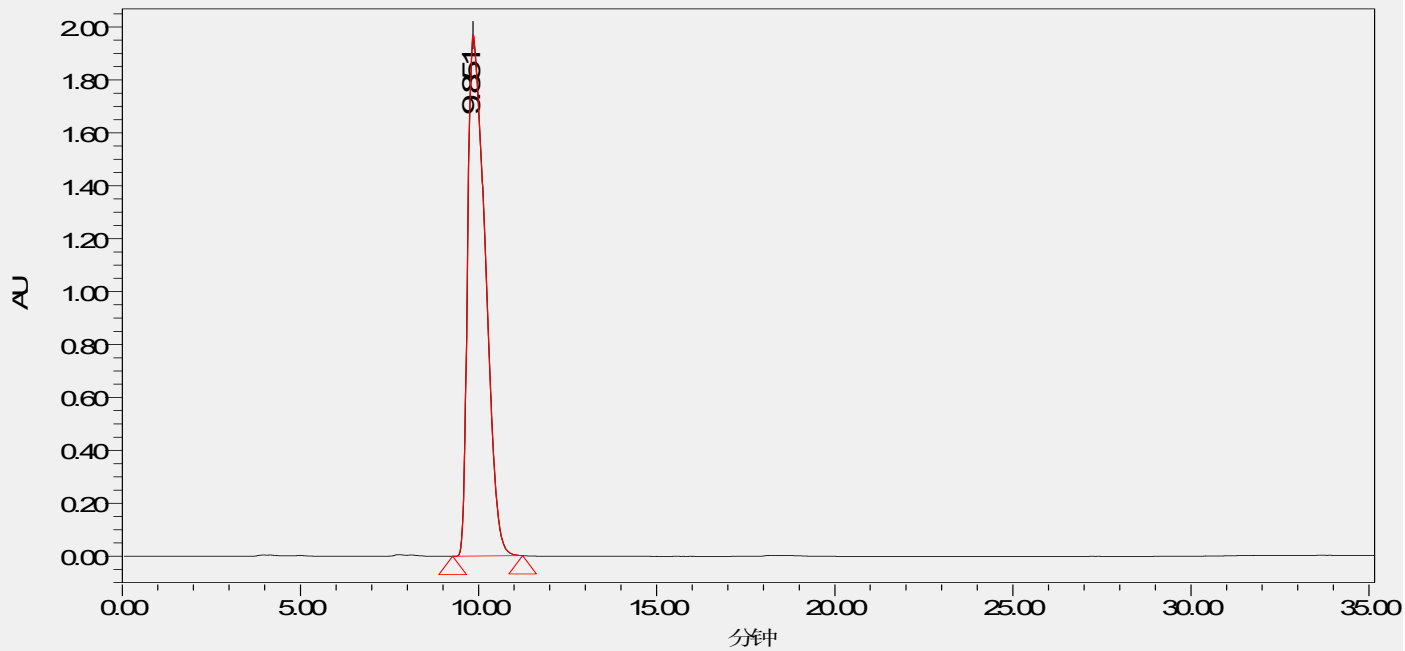

\begin{tabular}{|c|c|c|c|c|c|}
\hline Peak No & Retention time & Area \% & Area \% Area & Height & Integral type \\
\hline 1 & 9.851 & 67600373 & 100.00 & 1969144 & bb \\
\hline
\end{tabular}




\section{Comparative Study of NDTP with Other Coupling Reagents}

The yield and reaction time of NDTP was compared with well-known coupling reagents (HATU, HBTU, DCC, EDC) and TCT (2,4,6-Trichloro-1,3,5-triazine, a similar activating agent) and TFT (2,4,6-Trifluro-1,3,5-triazine) in the synthesis of 3ea on the gram scale.

Method (HATU, HBTU, EDC): A $100 \mathrm{~mL}$ round-bottom flask equipped with a stir bar was charged with Fmoc-L-Gln(Trt)-OH (3.0 mmol), L-Alanine methyl ester hydrochloride (3.0 mmol), the respective coupling reagent $(3.0 \mathrm{mmol}), \mathrm{DCM}(30 \mathrm{~mL})$ and DIEA $(9.0 \mathrm{mmol})$. The reaction mixture was stirred at room temperature for 30 minutes, then poured into $50 \mathrm{~mL}$ water and extracted and washed with DCM $(3 \times 20.0 \mathrm{~mL})$. The organic layers were combined, washed with brine, dried with $\mathrm{MgSO}_{4}$, concentrated under vacuum and purified by flash silica gel chromatography to afford corresponding dipeptide 3ea.

Method (DCC): A $100 \mathrm{~mL}$ round-bottom flask equipped with a stir bar was charged with Fmoc-L-Gln(Trt)-OH (3.0 mmol), L-Alanine methyl ester hydrochloride (3.0 mmol), DCC (3.0 $\mathrm{mmol})$, DCM $(30 \mathrm{~mL})$ and DIEA $(3.0 \mathrm{mmol})$. The reaction mixture was stirred at room temperature for 30 minutes, then concentrated under vacuum and purified by flash silica gel chromatography to afford corresponding dipeptide $\mathbf{3 e a}$.

Method (TCT, TFT): A $100 \mathrm{~mL}$ round-bottom flask equipped with a stir bar was charged with Fmoc-L-Gln(Trt)-OH (3.0 mmol), L-Alanine methyl ester hydrochloride (3.0 mmol), the coupling reagent $(1.0 \mathrm{mmol})$, DCM $(30 \mathrm{~mL})$ and DIEA $(9.0 \mathrm{mmol})$. The reaction mixture was stirred at room temperature for 30 minutes, then concentrated under vacuum and purified by flash silica gel chromatography to afford corresponding dipeptide 3ea.

Method (NDTP): A $100 \mathrm{~mL}$ round-bottom flask equipped with a stir bar was charged with Fmoc-L-Gln(Trt)-OH (3.0 mmol), L-Alanine methyl ester hydrochloride (3.0 mmol), DIEA (9.0 mmol) and MeCN (20 mL), the solution of NDTP $(3 \mathrm{mmol})$ in $\mathrm{MeCN}(10 \mathrm{~mL})$ was added and stirred for 1 minute at room temperature, tnen concentrated under vacuum and purified by flash silica gel chromatography to afford corresponding dipeptide 3ea. 
Table S2. Comparative study of NDTP and other coupling reagents<smiles>CCNC(=O)CCC(NCCF)C(=O)O</smiles><smiles>COC(=O)C(C)N</smiles><smiles>CCNC(=O)CCC(NC(F)F)C(=O)N[C@@H](C)C(=O)OC</smiles>

\begin{tabular}{ccccc}
\hline entry & coupling agent & solvent & time & yield \\
\hline 1 & HATU & DCM & $30 \mathrm{~min}$ & $96 \%$ \\
2 & HBTU & DCM & $30 \mathrm{~min}$ & $92 \%$ \\
3 & DCC & DCM & $30 \mathrm{~min}$ & $87 \%$ \\
4 & EDC & DCM & $30 \mathrm{~min}$ & $34 \%$ \\
5 & TCT & DCM & $30 \mathrm{~min}$ & $16 \%$ \\
6 & TFT & DCM & $30 \mathrm{~min}$ & $7 \%$ \\
7 & NDTP & MeCN & $1 \mathrm{~min}$ & $92 \%$ \\
\hline
\end{tabular}

The reaction was also monitored by analytic RP-HPLC as follows:

The coupling reactions were conducted the same as above methods, otherwise that they were interrupted with acetic acid $(3 \mathrm{~mL})$ when the mixture was stirred at room temperature for 1 minute. The reaction mixture was then imediately diluted and analyzed by analytic RP-HPLC.

Analyzing Method: corresponding analyzing was carried out on Waters e2695/2998 using XBridge ${ }^{\circledR}$ Peptide BEH C18 $(10 \mu \mathrm{m}, 4.6 \mathrm{~mm}$ x 250mm $)$ Column.

\begin{tabular}{cccc}
\hline Time (min) & A (\%) & C (\%) & Flow (mL/min) \\
\hline 0.0 & 35.0 & 65.0 & 1.0 \\
20.0 & 95.0 & 5.0 & 1.0 \\
20.5 & 100.0 & 0.0 & 1.0 \\
25.0 & 100.0 & 0.0 & 1.0 \\
26.0 & 35.0 & 65.0 & 1.0
\end{tabular}

Linear gradients of $\mathrm{HPLC}$ using $\mathrm{A}: \mathrm{MeCN}\left(0.1 \% \mathrm{CF}_{3} \mathrm{COOH}\right)$ and $\mathrm{B}: \mathrm{H}_{2} \mathrm{O}\left(0.1 \% \mathrm{CF}_{3} \mathrm{COOH}\right)$. 

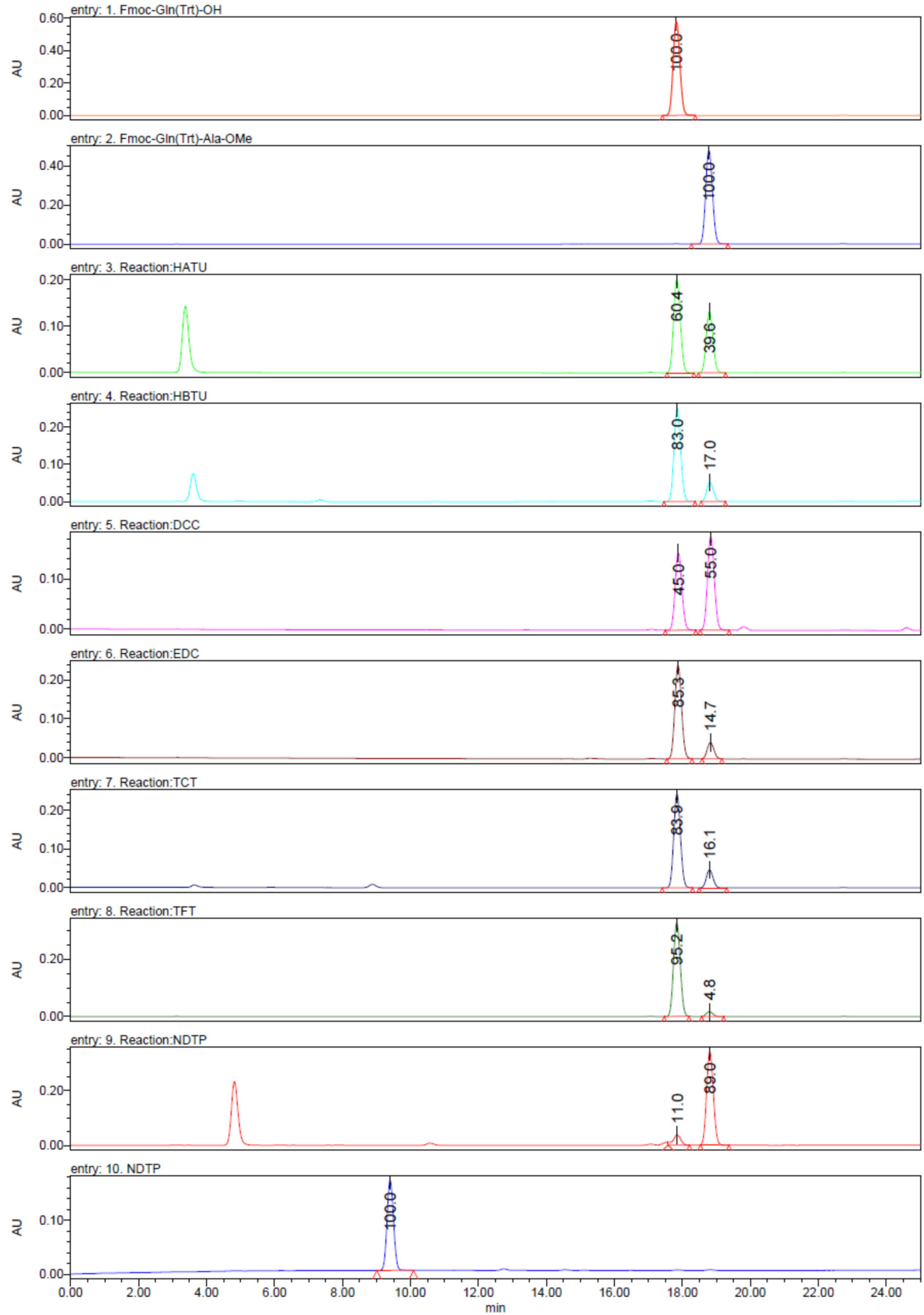


\section{Mechanical study}

To gain insights into the reaction, we carried out some control experiments. To a solution of benzoic acid $(0.2 \mathrm{mmol})$ with the presence of 0.2 eq DABCO in $\mathrm{MeCN}(0.5 \mathrm{~mL})$ was added NDTP $(0.2 \mathrm{mmol})$, the mixture was stirred $30 \mathrm{~s}$ and isolated by flash column chromatography (EtOAc: hexane $=1: 10)$ to afford the key intermediate benzoyl thiocyanide.

we also synthesized benzoyl thiocyanide according to the previous report ${ }^{l}$. To a solution of benzoyl chloride $(2.0 \mathrm{mmol})$ in acetone $(5.0 \mathrm{~mL})$ was added $\mathrm{NH}_{4} \mathrm{SCN}(2.0 \mathrm{mmol})$ at room temperature and stirred for $5 \mathrm{~min}$. The white precipitate of $\mathrm{NH}_{4} \mathrm{Cl}$ was filtered off, the solvent was evaporated under reduced pressure and purified by flash column chromatography (EtOAc: hexane $=1: 10$ ) to afford benzoyl thiocyanide, the HRMS and NMR was same with the intermediate.

Then, 1.0 eq DABCO and phenethylamine was added to the solution of benzoyl thiocyanide in $\mathrm{MeCN}$, the benzoyl thiocyanide intermediate successfully resulted to the amide product $\mathbf{4 f}$.

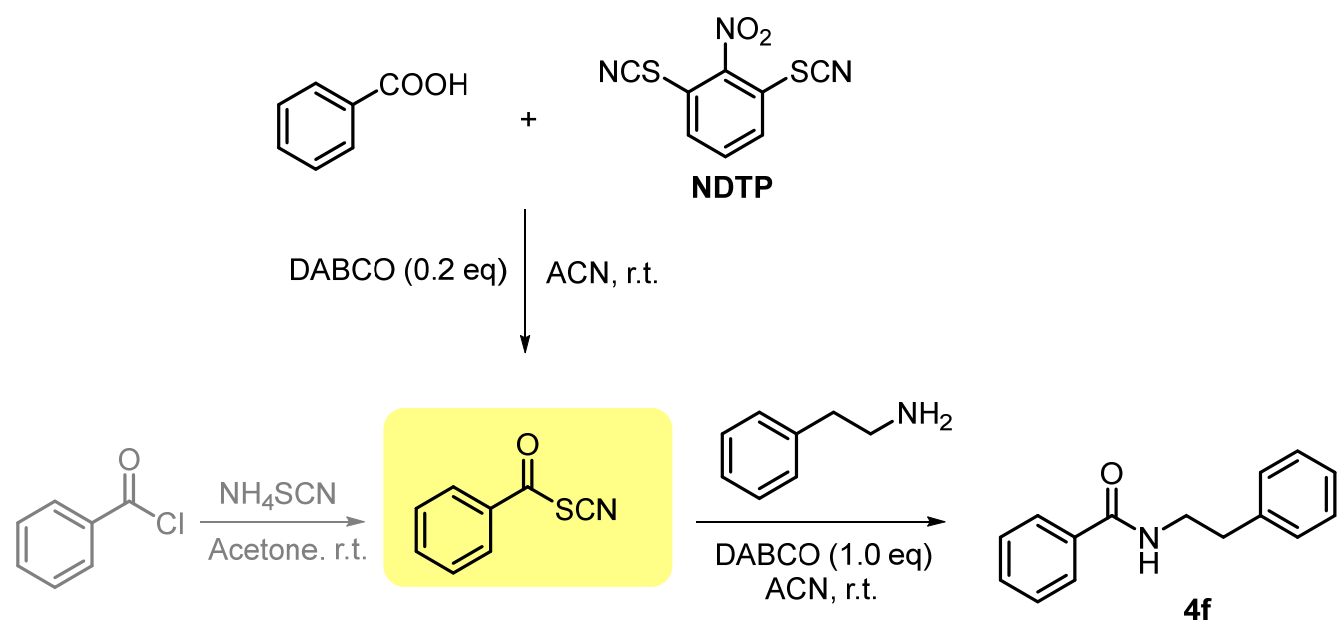

1. Khairul, W. M.; Mokthar, K. A.; Isa, M. I. N.; Samsudin, A. S.; Adli, H. K.; Ghazali, S. R.; Daud, A. I. Phosphorus, Sulfur, and Silicon and the Related Elements, 2014, 189, 640-651. 


\section{NDTP Recycling Procedure}

NDTP $(2.0 \mathrm{mmol})$ was added to a solution of phenylacetic acid $(2.0 \mathrm{mmol})$, DABCO $(6.0 \mathrm{mmol})$ and 1-Naphthylamine $(2.0 \mathrm{mmol})$ in $\mathrm{MeCN}(10 \mathrm{~mL})$. The mixture was stirred for $1 \mathrm{~min}$ and then the precipitate was filtered off, washed with $\mathrm{MeCN}$. The filtrate was evaporated under reduced pressure and purified by flash chromatography to obtain the product $\mathbf{4 j}$ ( $522 \mathrm{mg}, 84 \%$ ). The solid was dried, then treated with $\mathrm{POCl}_{3}$ under reflux for 3 hours, then the mixture was cooled to room temperature and poured into ice water, the white precipitate was collected to afford 4,6-dichloro-5nitropyrimidine (216 mg, 56\%), it can easily be transformed into NDTP again.

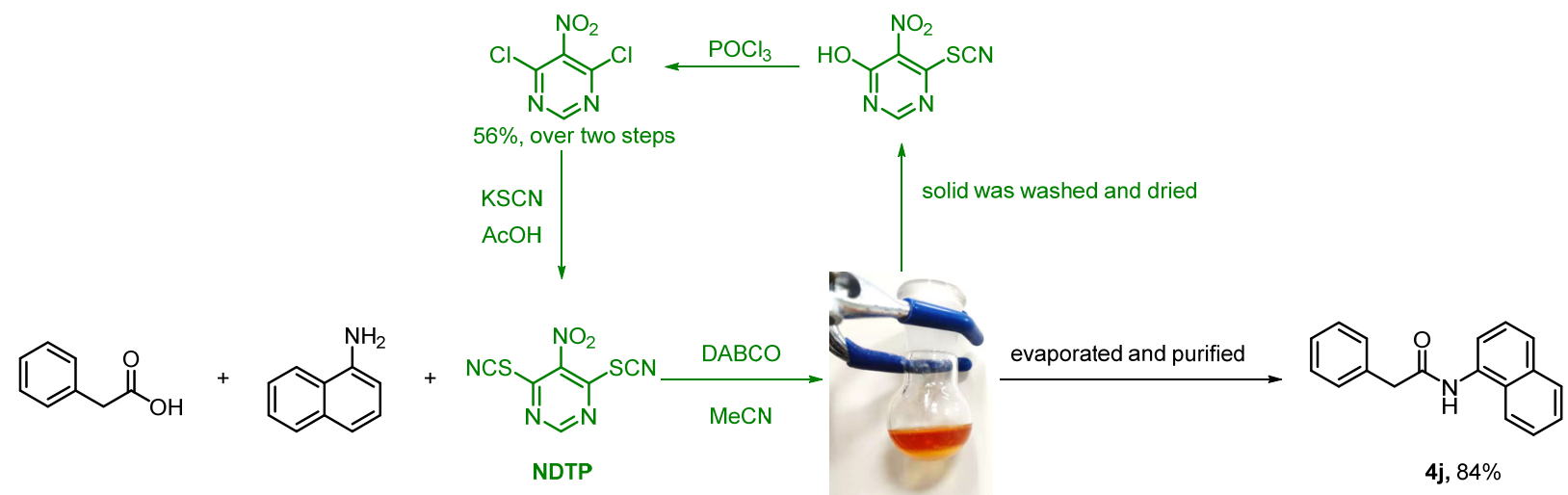

Fig. S1 


\section{Procedures for the Synthesis of Peptides on Resin (SPPS)}

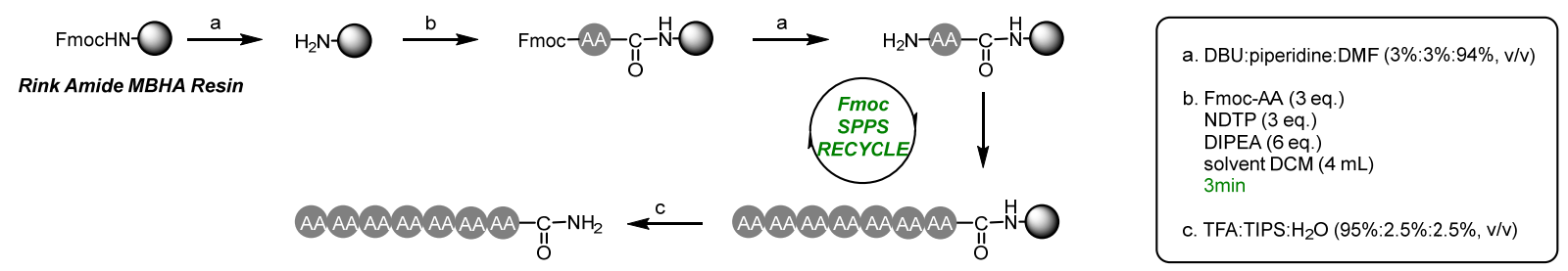

\section{General procedure for incorporating the first amino acid on the solid support}

Into a $20 \mathrm{~mL}$ filtration tube with a stir bar (350 r/min) was added rink amide MBHA resin $(0.1$ mmol). The resin was successively swollen in DCM $(5 \mathrm{~mL})$ for $30 \mathrm{~min}$, then drained and treated with a solution $(4.0 \mathrm{~mL})$ of DBU: piperidine: $\operatorname{DMF}(3: 3: 94, \mathrm{v} / \mathrm{v})$ for $5 \mathrm{~min}$ before being drained. The solution was added again for $15 \mathrm{~min}$, then drained and the resin was washed with DMF $(2 \times$ $4.0 \mathrm{~mL})$ and DCM $(3 \times 4.0 \mathrm{~mL})$. The solution of Fmoc-protected amino acid $(0.3 \mathrm{mmol})$ and DIEA $(0.6 \mathrm{mmol})$ in DCM $(2 \mathrm{~mL})$ was added into the tube and stirred $(350 \mathrm{r} / \mathrm{min})$, then the solution of NDTP $(0.3 \mathrm{mmol})$ in DCM $(2 \mathrm{~mL})$ was gradually dripped into the tube within 2 minutes using Syringe Pump. After stirring for 1 minute, the resin was washed with DCM $(3 \times 4.0 \mathrm{~mL})$ and DMF $(2 \times 4.0 \mathrm{~mL})$ before drained.

\section{General procedure for removing Fmoc-protection}

The solution $(4.0 \mathrm{~mL})$ of DBU: piperidine: $\operatorname{DMF}(3: 3: 94, \mathrm{v} / \mathrm{v})$ was added to the filtration tube for $5 \mathrm{~min}$ before being drained, repeated the procedure for $15 \mathrm{~min}$. Then the resin was drained and washed with DMF $(2 \times 4.0 \mathrm{~mL})$ and DCM $(3 \times 4.0 \mathrm{~mL})$.

\section{General procedure for peptide elongation}

The solution of Fmoc-protected amino acid $(0.3 \mathrm{mmol})$ and DIEA $(0.6 \mathrm{mmol})$ in DCM $(2 \mathrm{~mL})$ was added and stirred $(350 \mathrm{r} / \mathrm{min})$, then the solution of NDTP $(0.3 \mathrm{mmol})$ in DCM $(2 \mathrm{~mL})$ was gradually dripped into the tube within 2 minutes using Syringe Pump. After stirring for 1 minute, the resin was washed with DCM $(3 \times 4.0 \mathrm{~mL})$ and DMF $(2 \times 4.0 \mathrm{~mL})$ before drained.

\section{General procedure of resin cleavage and purification}

The resin was thoroughly rinsed with DMF $(4 \times 4 \mathrm{~mL})$, DCM $(2 \times 4 \mathrm{~mL}), \mathrm{MeOH}(1 \times 4 \mathrm{~mL})$, $\mathrm{DCM}(1 \times 4 \mathrm{~mL})$ and $\mathrm{MeOH}(2 \times 4 \mathrm{~mL})$. After completion of the procedure, the resin was treated 
with a solution of TFA/TIPS/ $\mathrm{H}_{2} \mathrm{O}(4.75 \mathrm{~mL} / 0.125 \mathrm{~mL} / 0.125 \mathrm{~mL})$ for $3 \mathrm{~h}$ to cleave the crude peptide product. The resin was then filtered and washed with pure TFA $(3 \times 2 \mathrm{~mL})$. The mixture was concentrated under reduced pressure, added $40 \mathrm{~mL}$ cold diethyl ether and pale yellow solid precipitated. The precipitate was dissolved in $\mathrm{H}_{2} \mathrm{O} / \mathrm{MeCN}$ and the ether layer was extracted with water. The aqueous solutions were combined, lyophilized and further purified by semi-preparative HPLC to yield the pure product 5 . 


\section{Characterization and Purity analysis for 5}

5a: H-Arg-Lys-Asp-Val-Tyr-NH2 (white solid, 37mg, 57\% yield). MS-API m/z: $[\mathrm{M}+\mathrm{H}]^{+}$calcd for $\mathrm{C}_{30} \mathrm{H}_{51} \mathrm{~N}_{10} \mathrm{O}_{8} 679.38$, found: 679.6.<smiles>CC(C)C(NC(=O)[C@H](CC(=O)O)NC(=O)[C@H](CCCCN)NC(=O)[C@H](N)CCCNC(=N)N)C(=O)N[C@@H](Cc1ccc(O)cc1)C(N)=O</smiles>

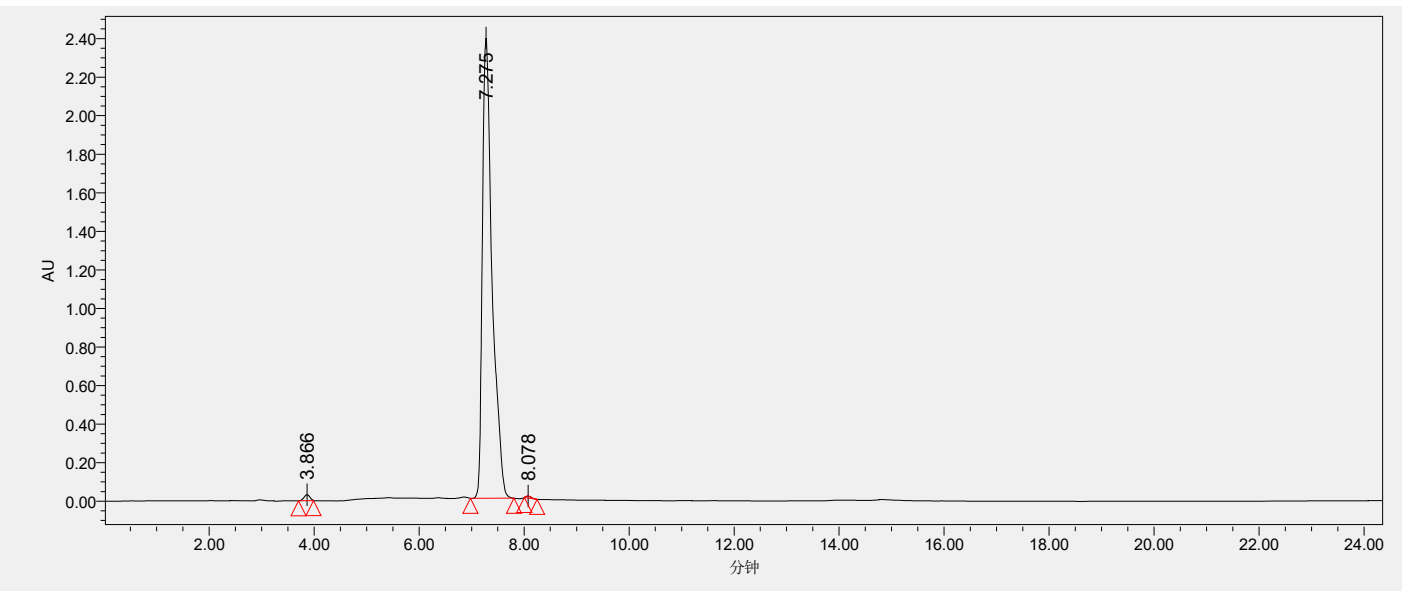

\begin{tabular}{|c|c|c|c|c|c|}
\hline & Retention time & Area & \% Area & Height & Type \\
\hline 1 & 3.866 & 241467 & 0.78 & 31094 & $\mathrm{bb}$ \\
\hline 2 & 7.275 & 30833704 & 99.00 & 2387887 & $\mathrm{bb}$ \\
\hline 3 & 8.078 & 69678 & 0.22 & 10290 & $\mathrm{bb}$ \\
\hline
\end{tabular}

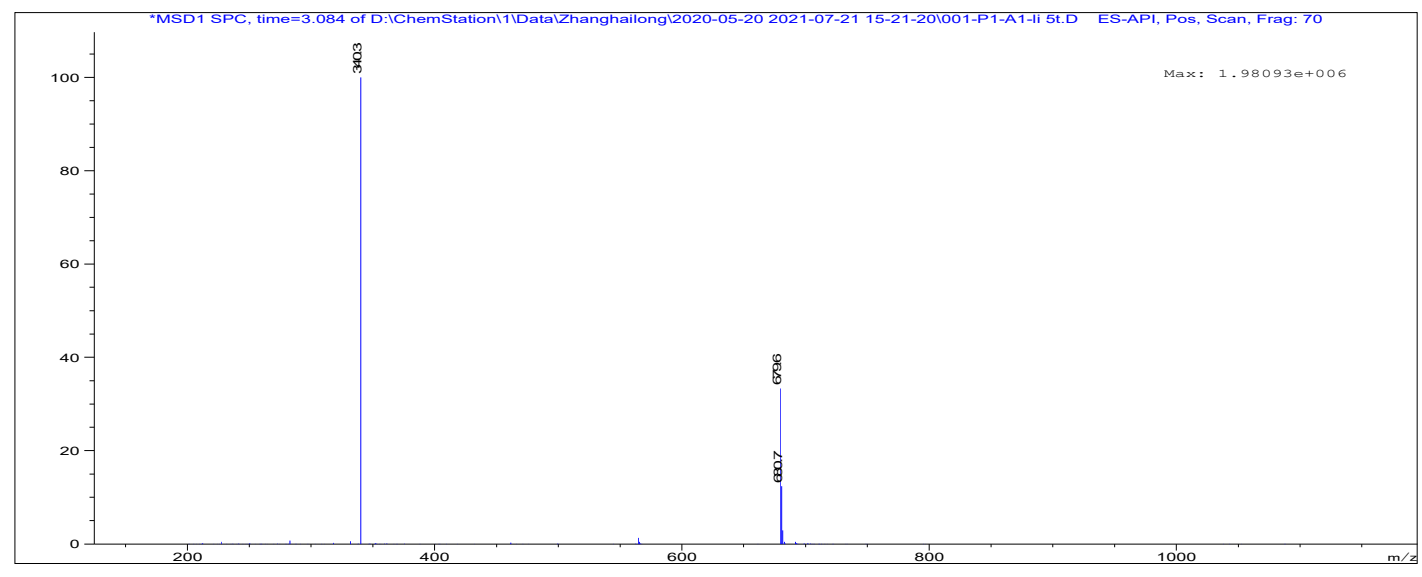


5b: H-Ser-Leu-Ile-Gly-Lys-Val-NH 2 (white solid, 20mg, 33\% yield). MS-API m/z: [M+H] $]^{+}$calcd for $\mathrm{C}_{28} \mathrm{H}_{55} \mathrm{~N}_{8} \mathrm{O}_{7}$ 615.42, found: 615.6.<smiles>CC[C@H](C)[C@H](NC(=O)[C@H](CC(C)C)NC(=O)C(N)CO)C(=O)NCC(=O)NC(CCCCN)C(=O)N[C@H](C(N)=O)C(C)C</smiles>

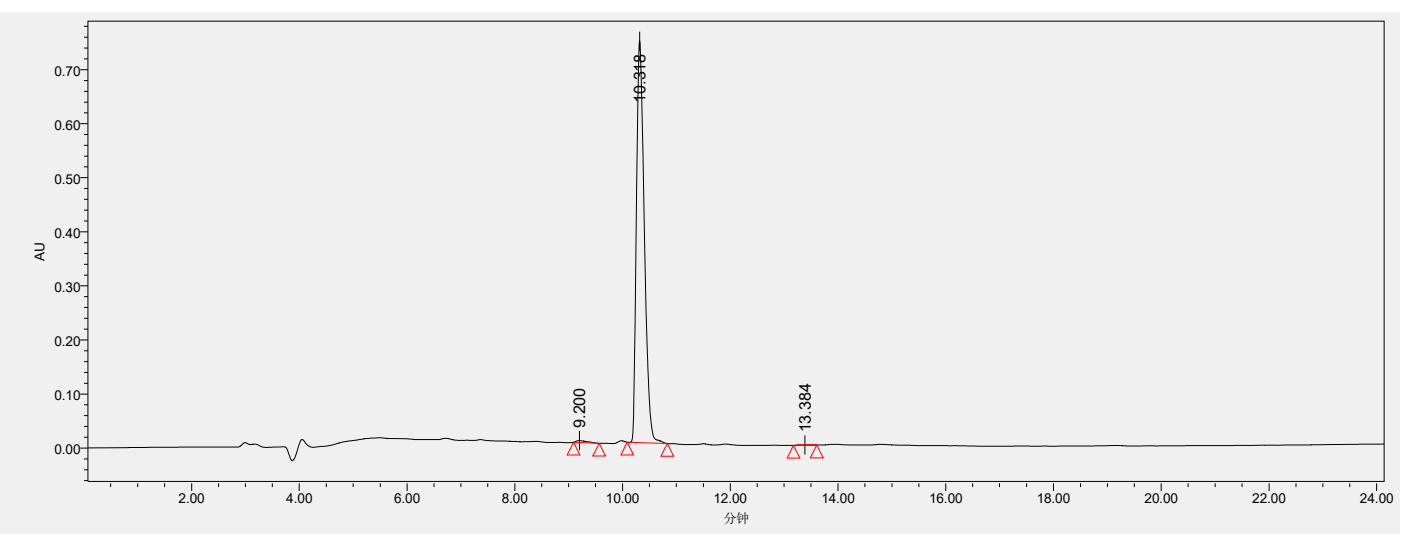

\begin{tabular}{|c|c|c|c|c|c|}
\hline & Retention time & Area & \% Area & Height & Type \\
\hline 1 & 9.200 & 49902 & 0.65 & 3468 & $\mathrm{bb}$ \\
\hline 2 & 10.318 & 7623602 & 99.25 & 743091 & $\mathrm{bb}$ \\
\hline 3 & 13.384 & 7492 & 0.10 & 482 & $\mathrm{bb}$ \\
\hline
\end{tabular}

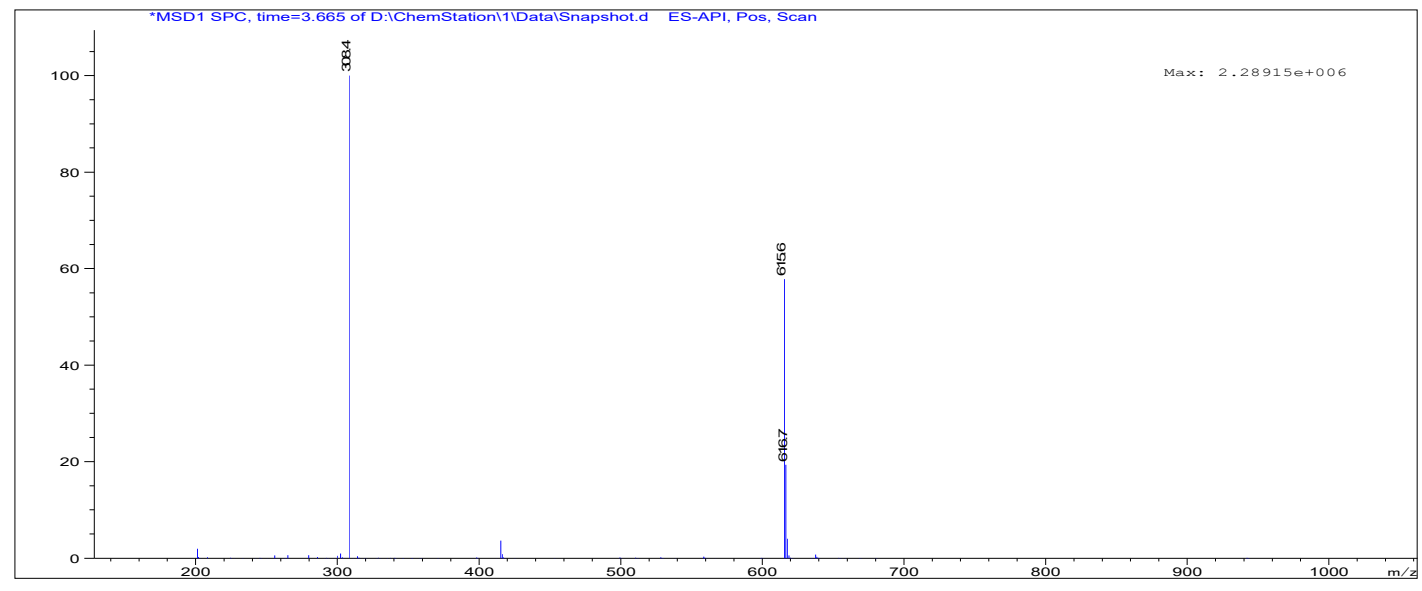


5c: H-Ile-Ala-Arg-Arg-His-Pro-Tyr-Phe-Leu-NH2 (white solid, 19mg, 16\% yield). MS-API m/z: $[\mathrm{M}+\mathrm{H}]^{+}$calcd for $\mathrm{C}_{56} \mathrm{H}_{87} \mathrm{~N}_{18} \mathrm{O}_{10} 1171.68$, found: 1173.0 .
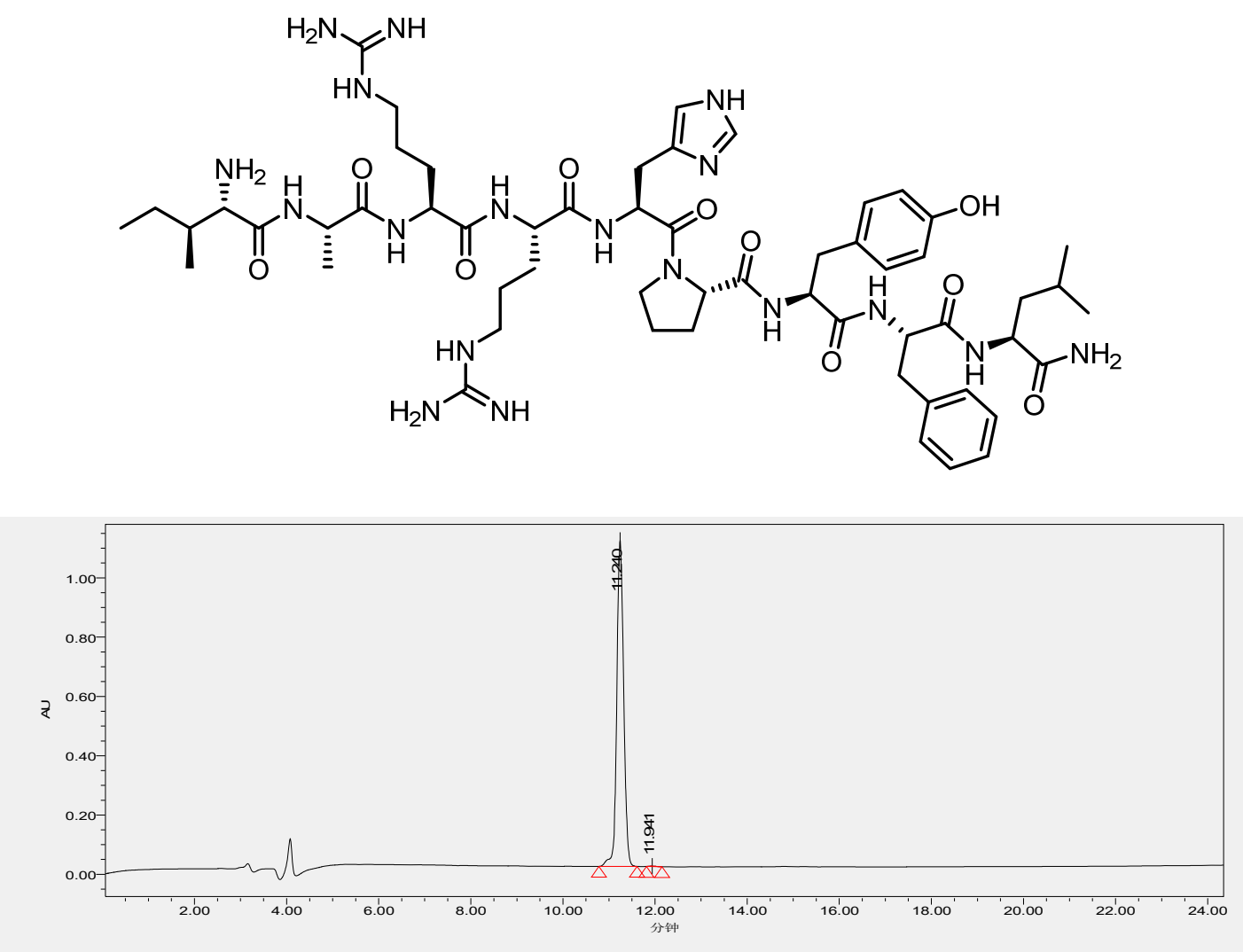

\begin{tabular}{|c|c|c|c|c|c|}
\hline & Retention time & Area & $\%$ Area & Height & Type \\
\hline 1 & 11.240 & 11413601 & 99.86 & 1099588 & $\mathrm{bb}$ \\
\hline 2 & 11.941 & 15478 & 0.14 & 1530 & $\mathrm{bb}$ \\
\hline
\end{tabular}

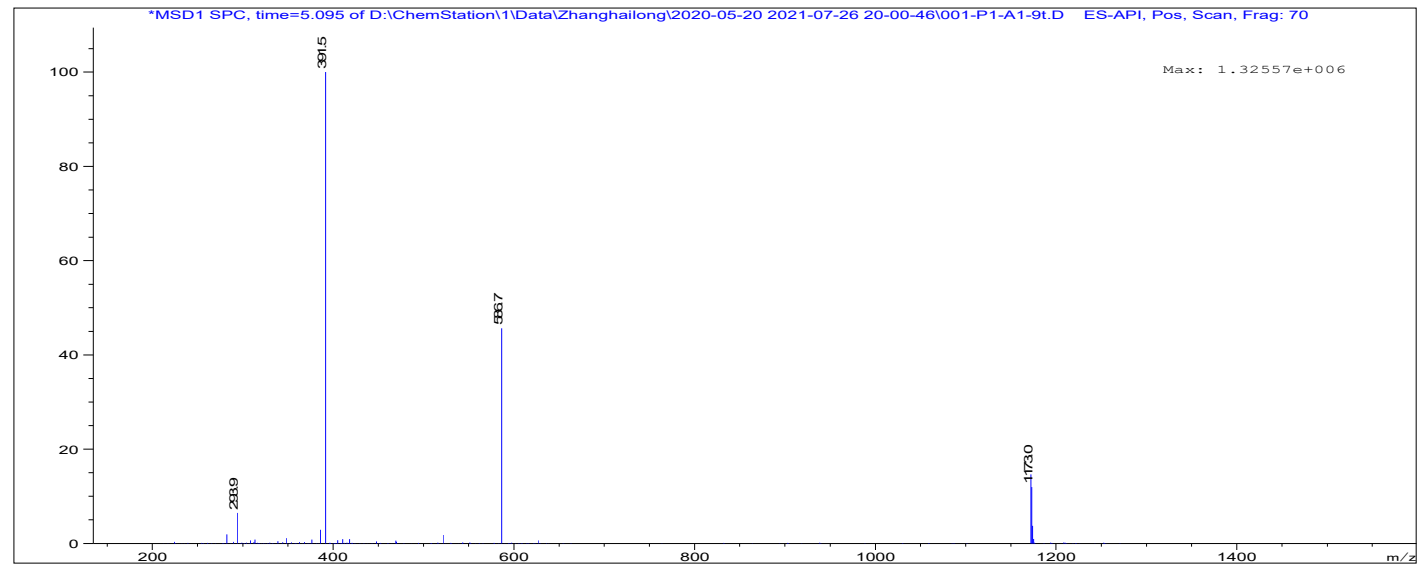


5d: H-Ala-Pro-Ser-Gly-Ala-Gln-Arg-Leu-Tyr-Gly-Phe-Gly-Leu-NH $\mathrm{N}_{2}$ (white solid, 26mg, 19\% yield). MS-API m/z: $[\mathrm{M}+\mathrm{H}]^{+}$calcd for $\mathrm{C}_{61} \mathrm{H}_{95} \mathrm{~N}_{18} \mathrm{O}_{16}$ 1335.71, found: 1336.1.
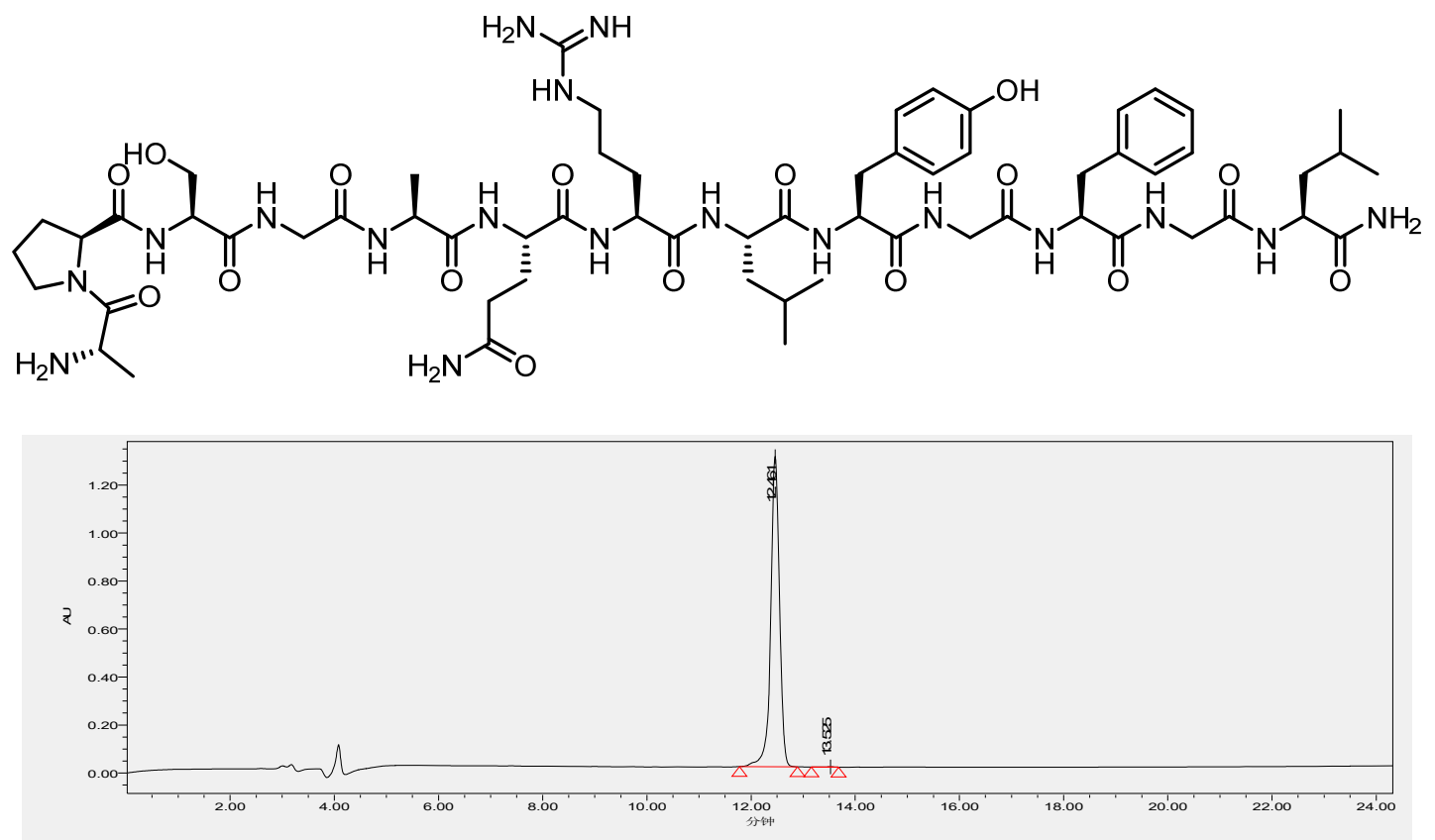

\begin{tabular}{|c|c|c|c|c|c|}
\hline & Retention time & Area & $\%$ Area & Height & Type \\
\hline 1 & 12.461 & 15199319 & 99.94 & 1292277 & $\mathrm{bb}$ \\
\hline 2 & 13.525 & 9843 & 0.06 & 954 & $\mathrm{bb}$ \\
\hline
\end{tabular}

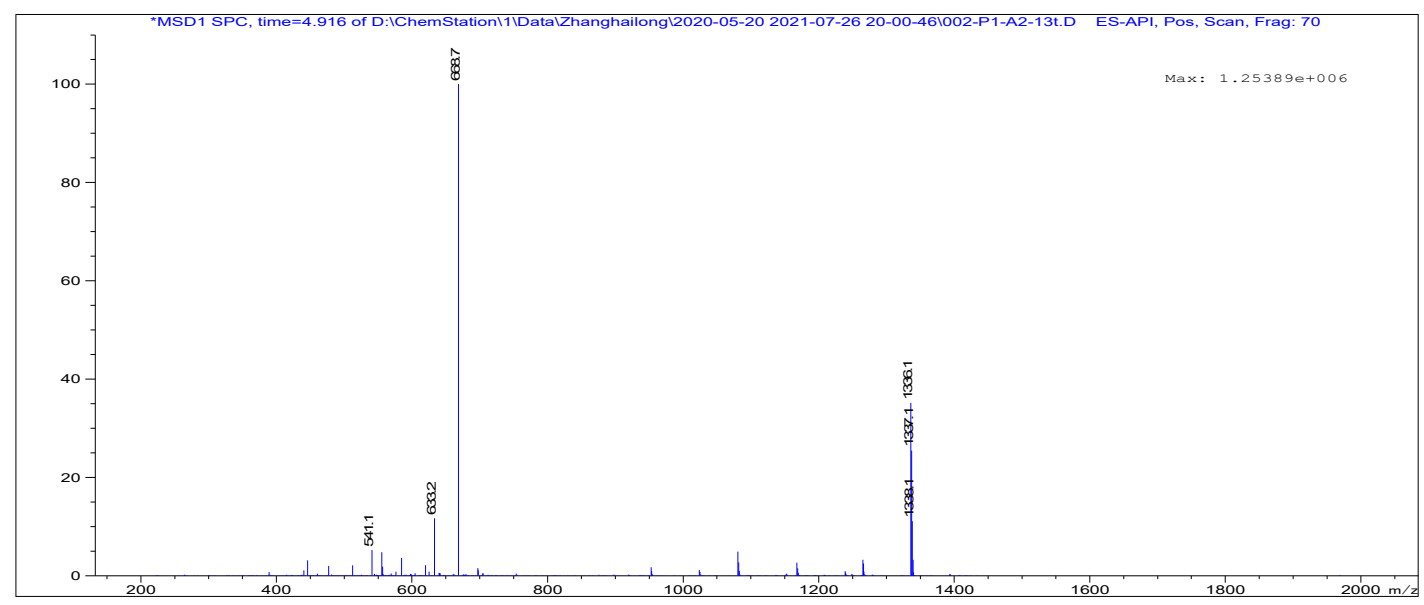




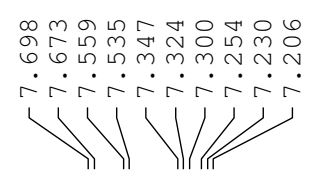

Fmochn $\overbrace{\text { H }}^{2}$

3aa, $300 \mathrm{MHz}, \mathrm{CDCl}_{3}$

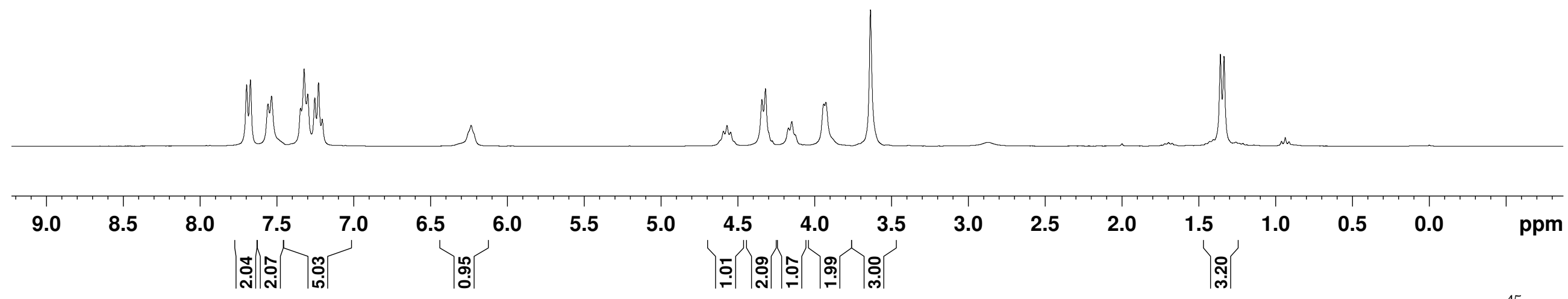


3aa, $75 \mathrm{MHz}, \mathrm{CDCl}_{3}$

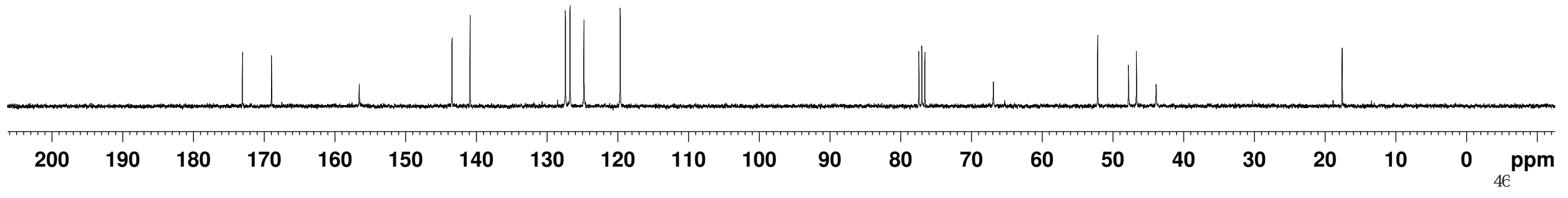




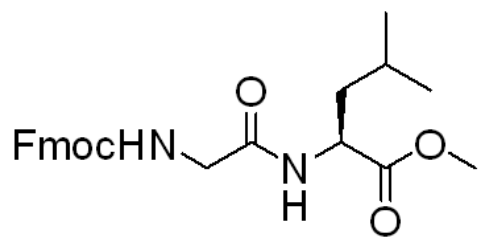

3ab, $300 \mathrm{MHz}, \mathrm{CDCl}_{3}$

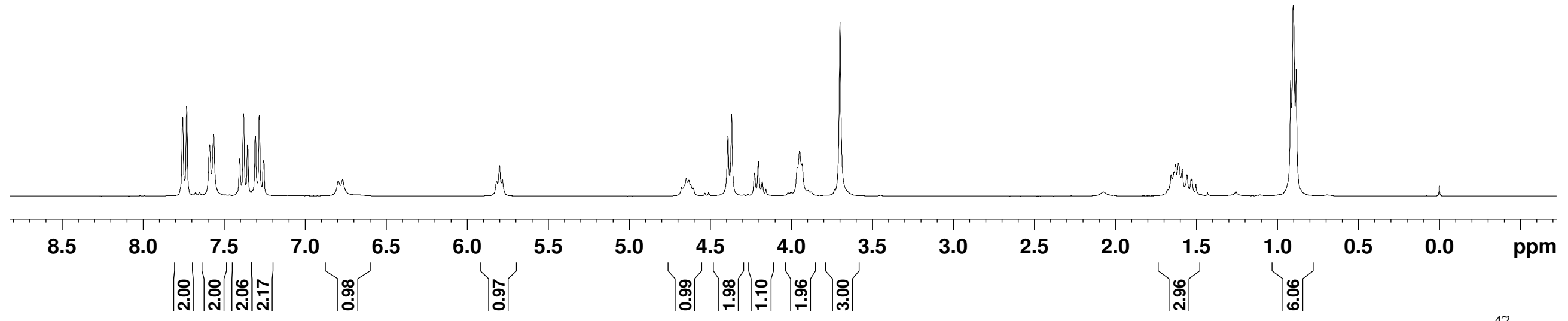


<smiles>COC(=O)C(CC(C)C)NC(=O)CNCC(F)F</smiles>

3ab, $75 \mathrm{MHz}, \mathrm{CDCl}_{3}$

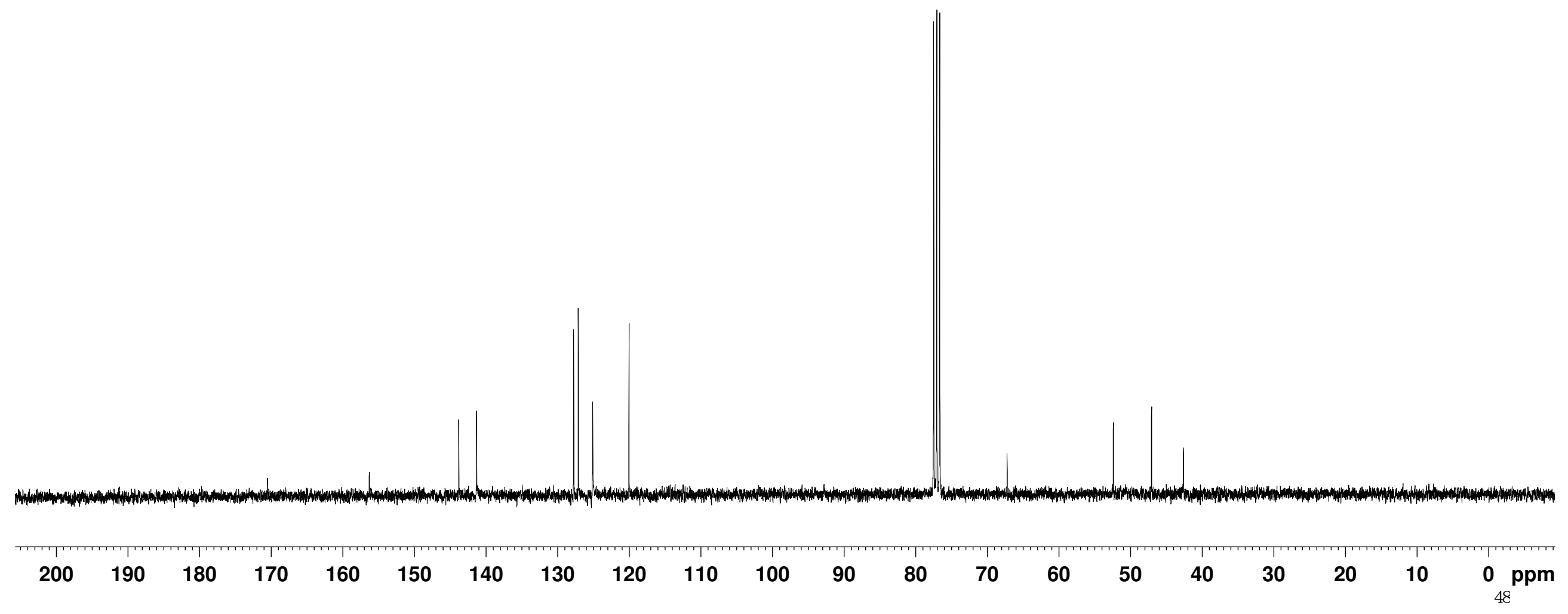



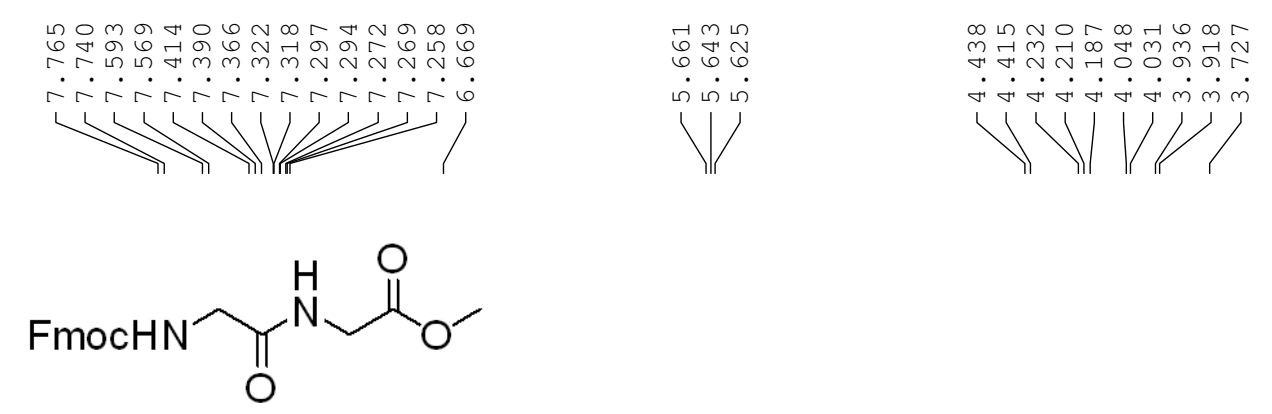

3ac, $300 \mathrm{MHz}, \mathrm{CDCl}_{3}$

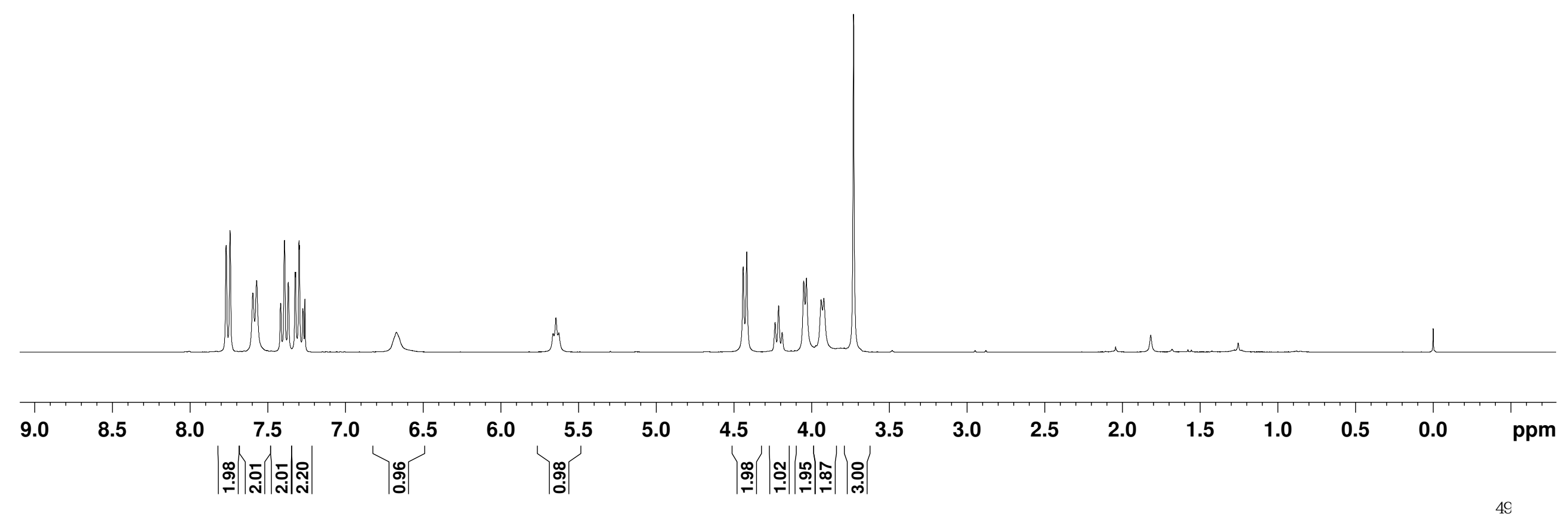




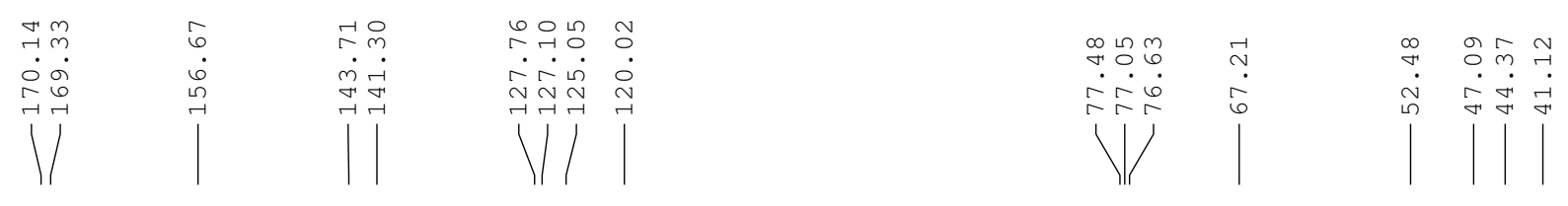<smiles>COC(=O)CNC(=O)CNC=C(F)F</smiles>

3ac, $75 \mathrm{MHz}, \mathrm{CDCl}_{3}$

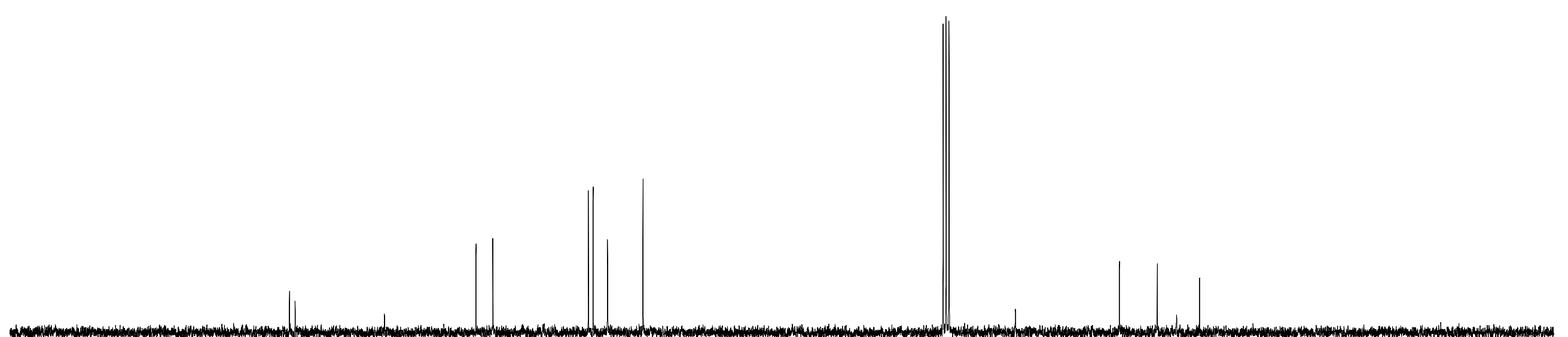




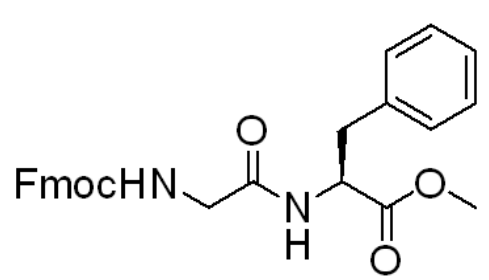

3ad, $300 \mathrm{MHz}, \mathrm{CDCl}_{3}$

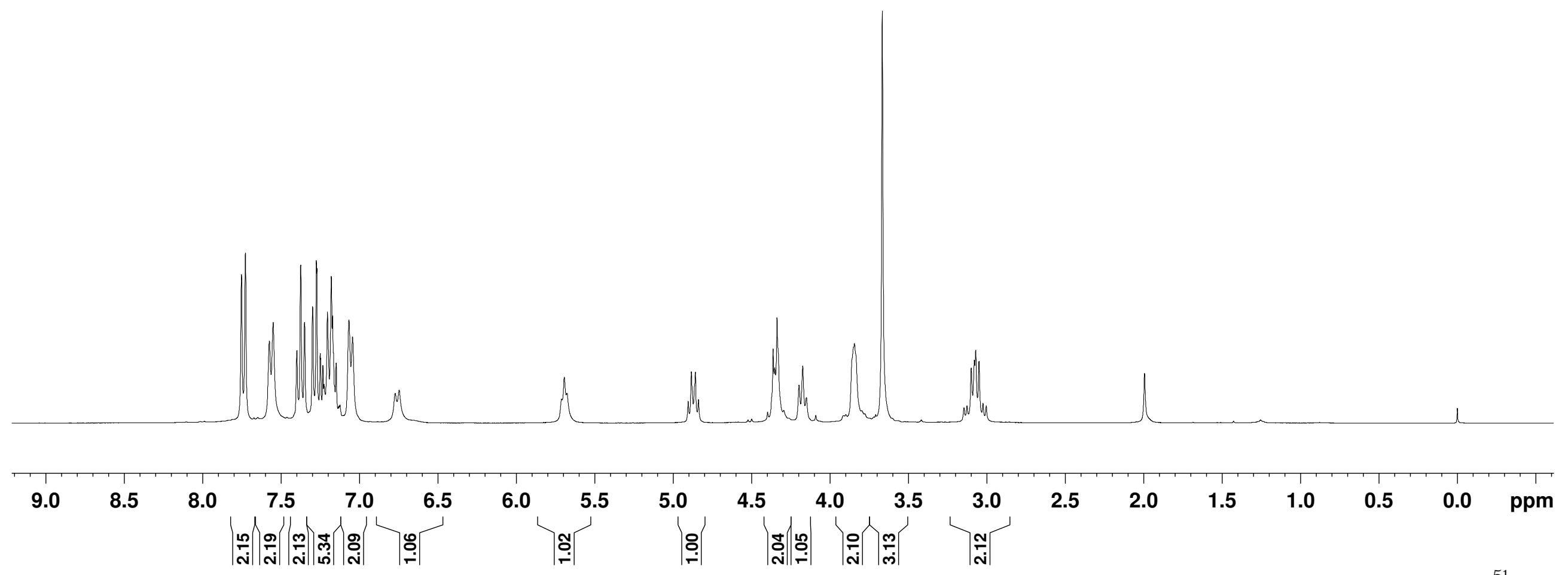




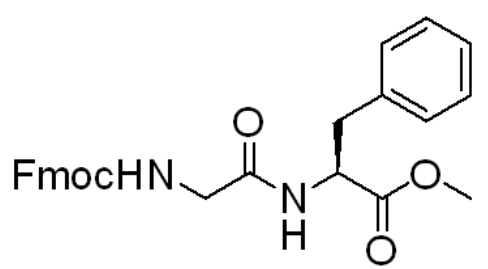

3ad, $75 \mathrm{MHz} \mathrm{CDCl}_{3}$

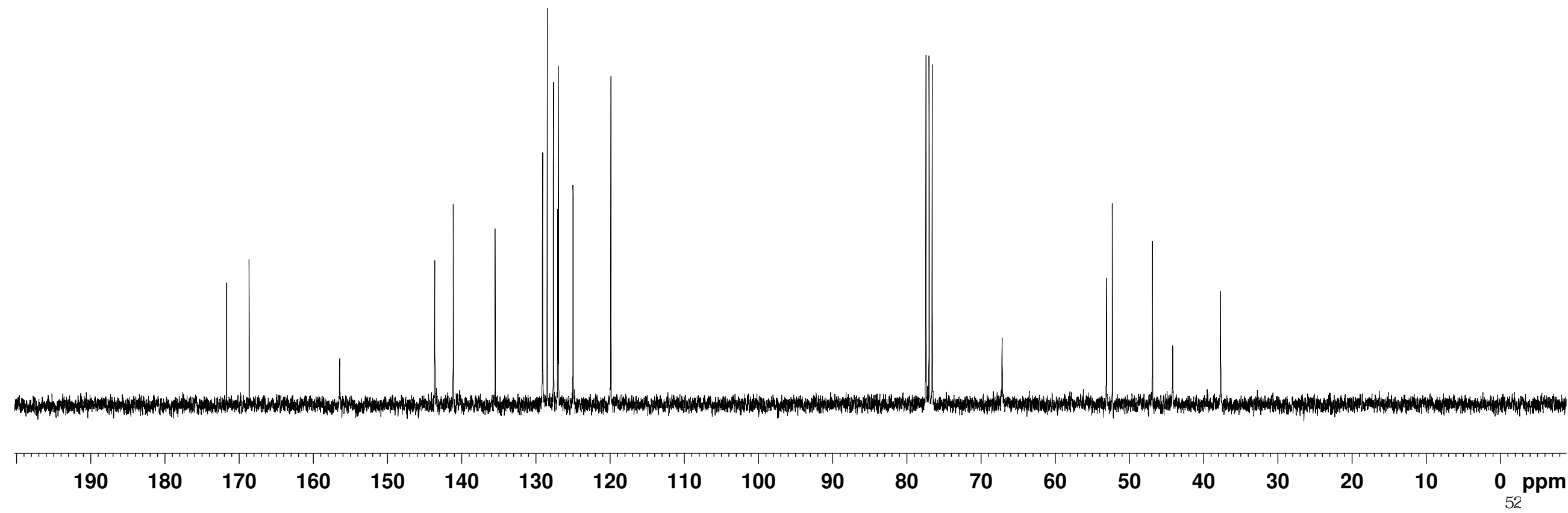



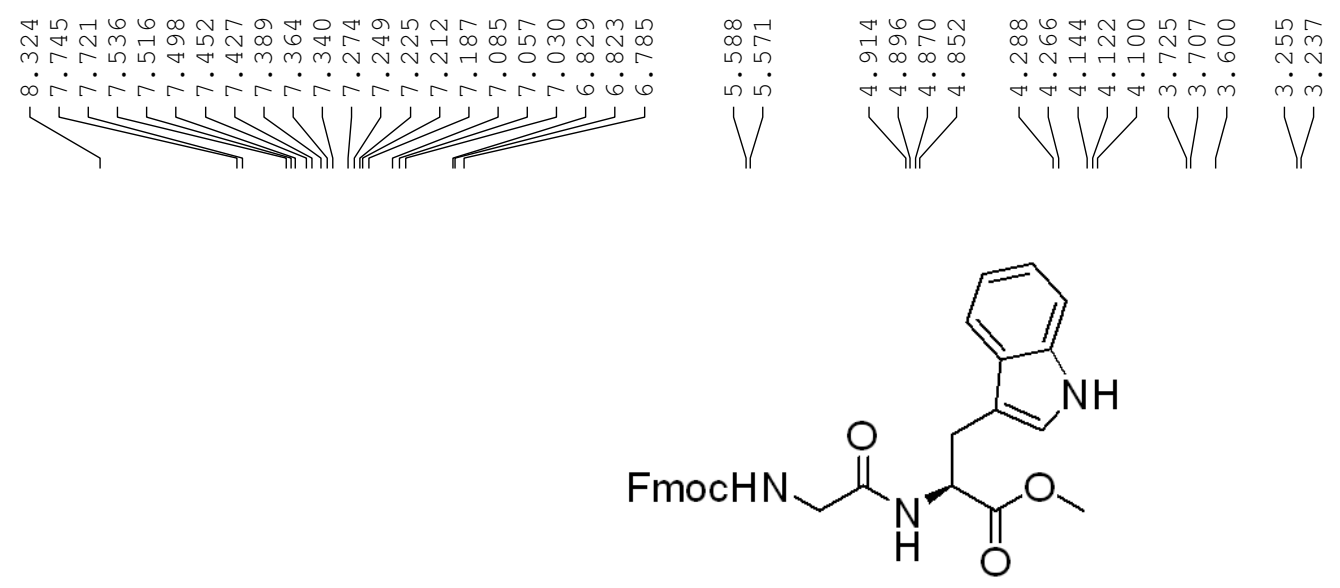

3ae, $300 \mathrm{MHz}, \mathrm{CDCl}_{3}$

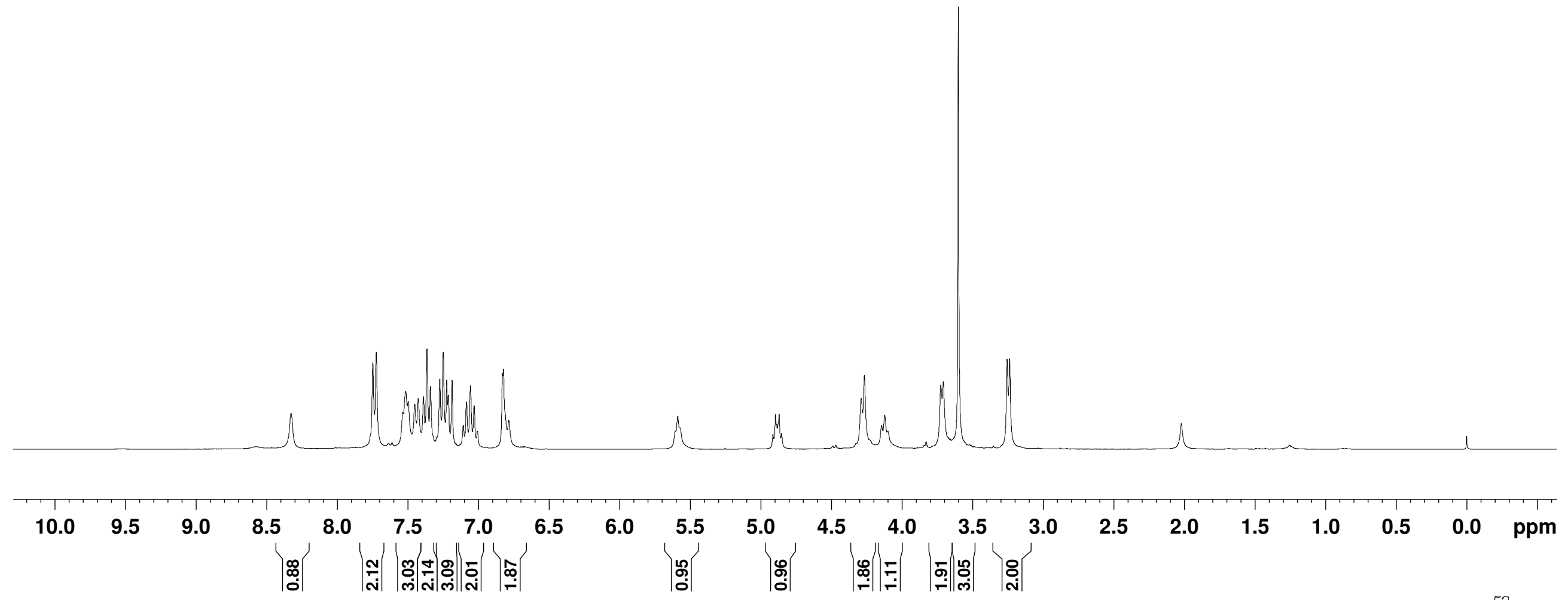




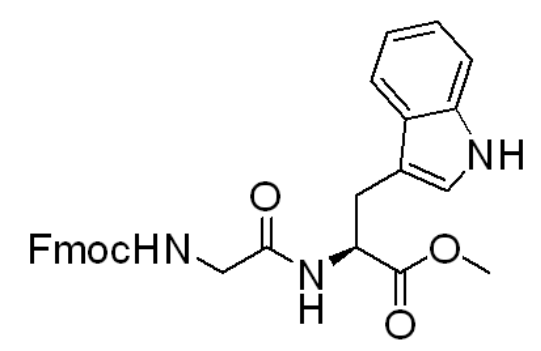

3ae, $75 \mathrm{MHz}, \mathrm{CDCl}_{3}$

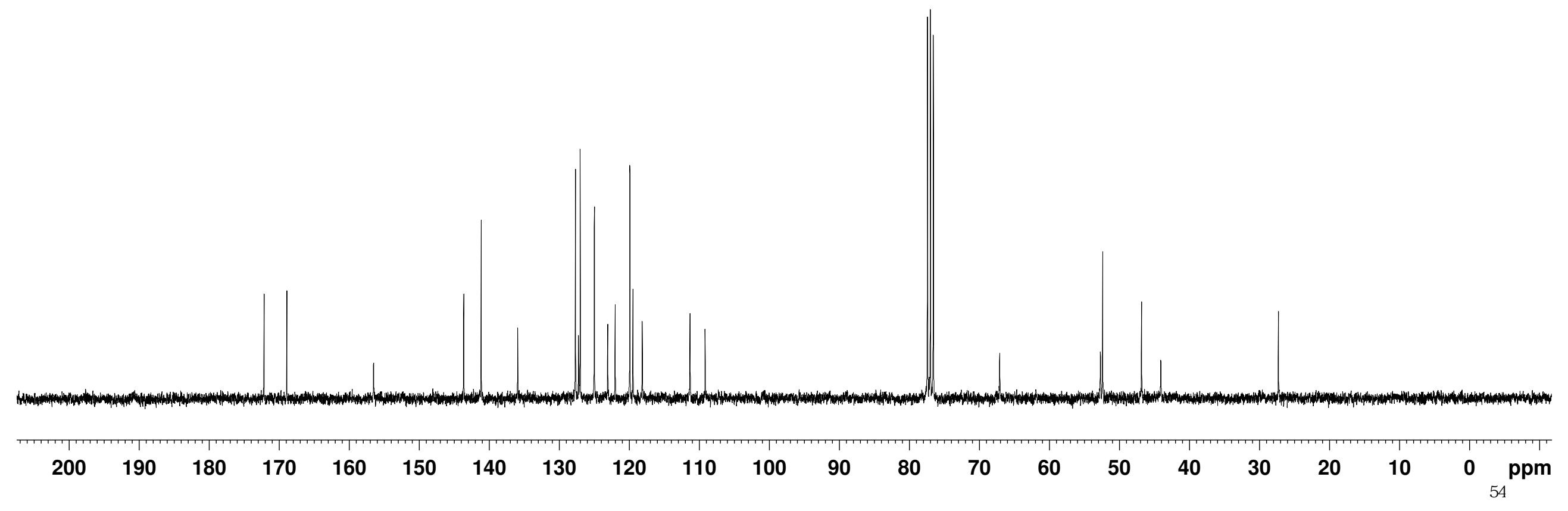




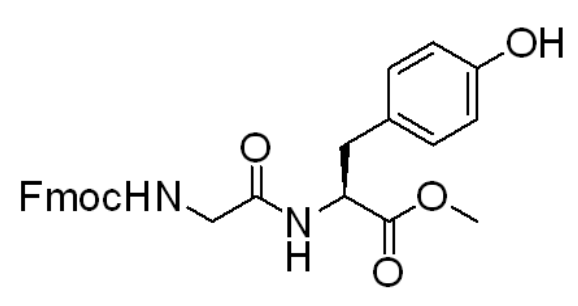

3af, $300 \mathrm{MHz}, \mathrm{MeOD}$

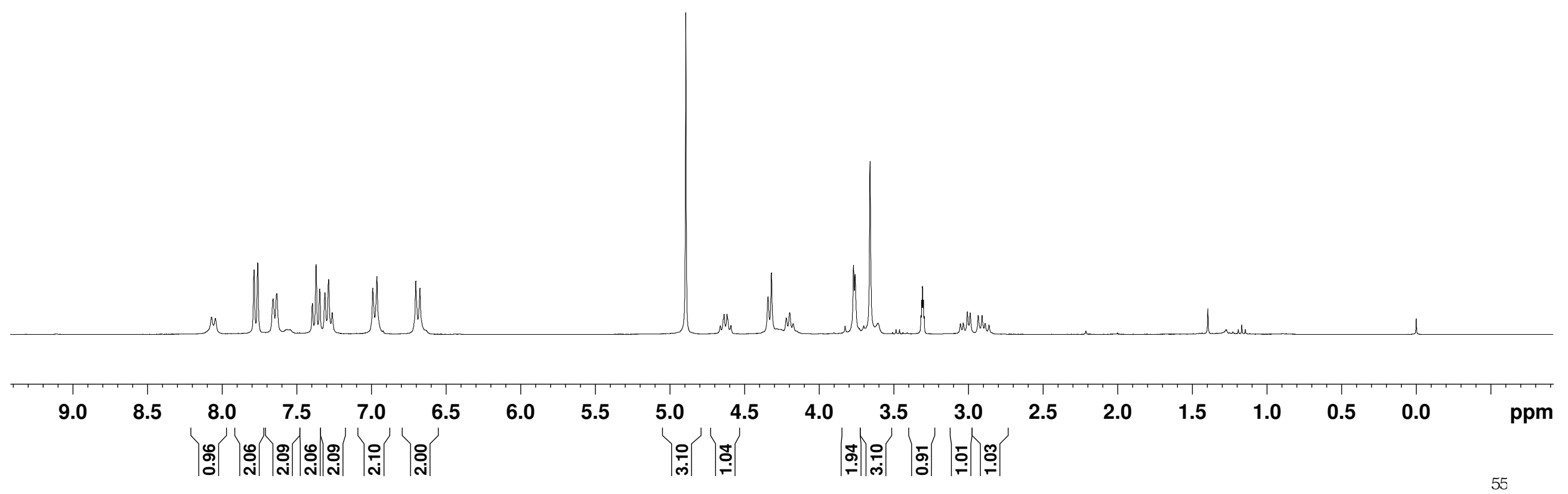




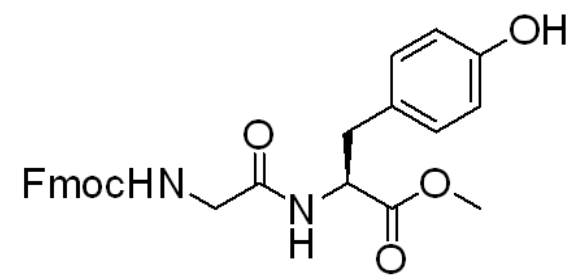

3af, $75 \mathrm{MHz}$, MeOD

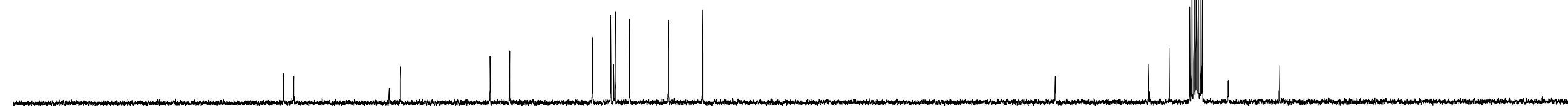




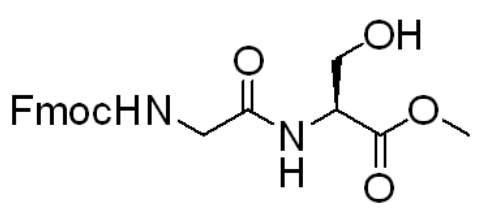

3ag, $300 \mathrm{MHz}, \mathrm{CDCl}_{3}$

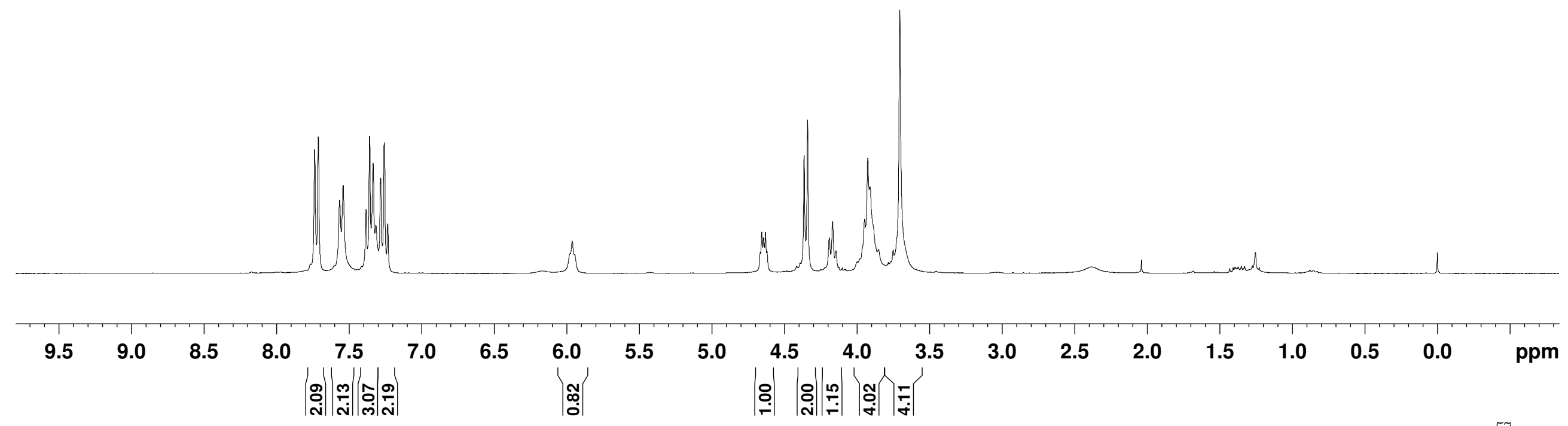




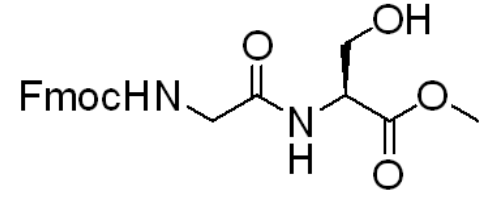

$3 \mathrm{ag}, 75 \mathrm{MHz}, \mathrm{CDCl}_{3}$

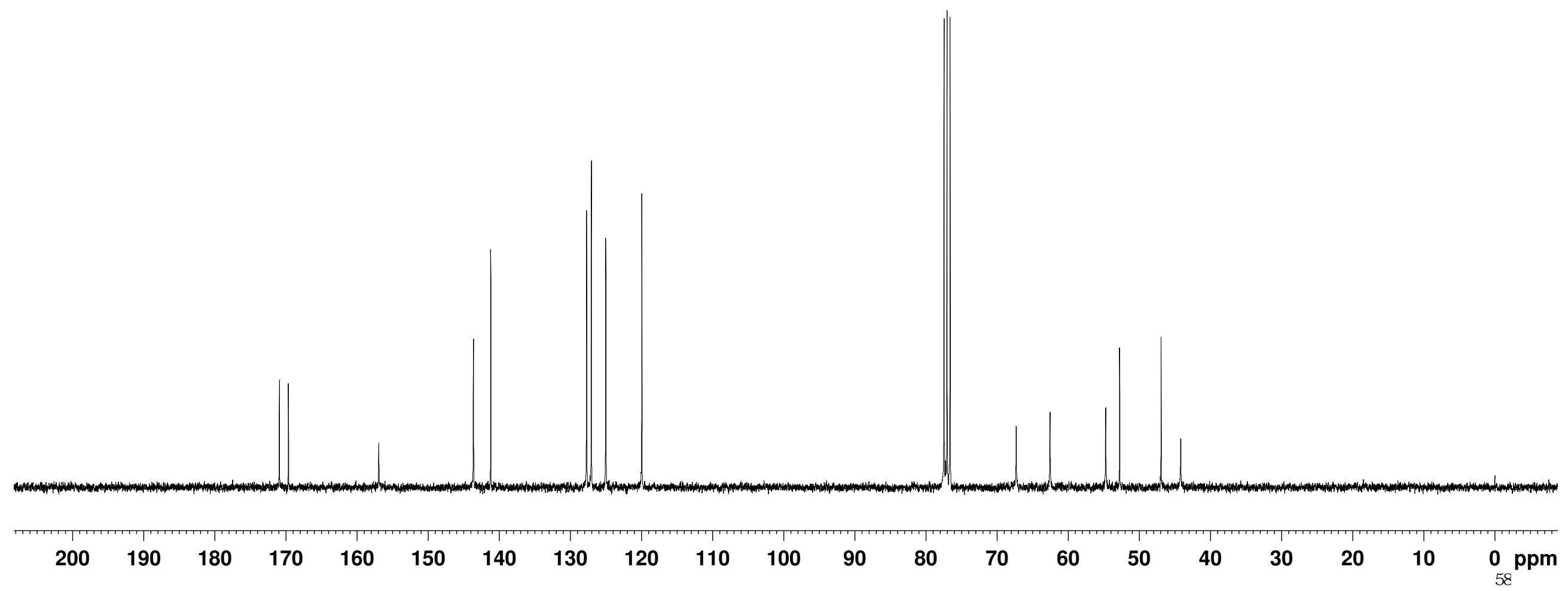




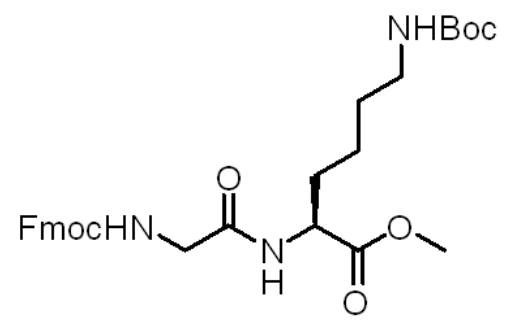

3ah, $300 \mathrm{MHz}, \mathrm{CDCl}_{3}$

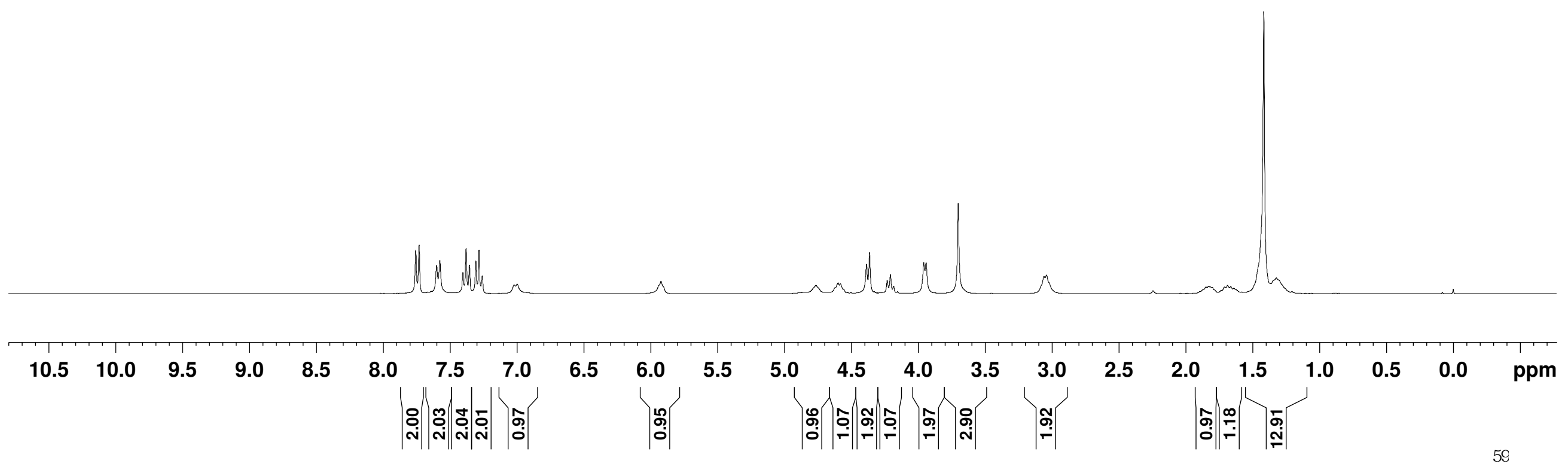




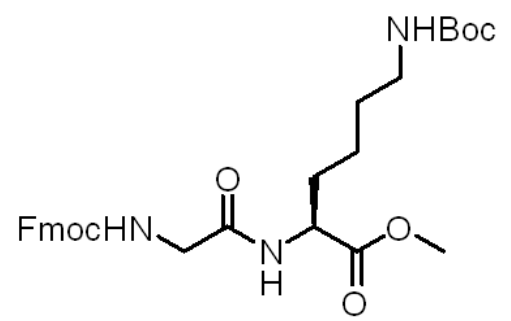

3ah, 75 MHz, CDCl

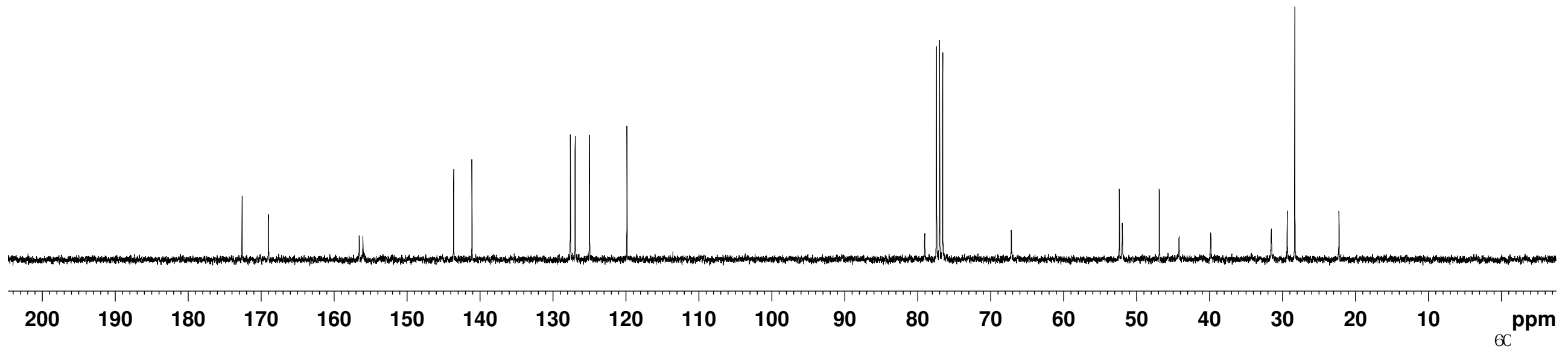



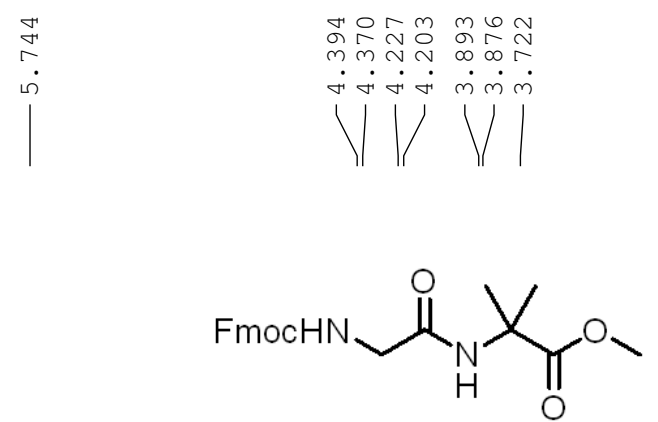

3ai, $300 \mathrm{MHz}, \mathrm{CDCl}_{3}$

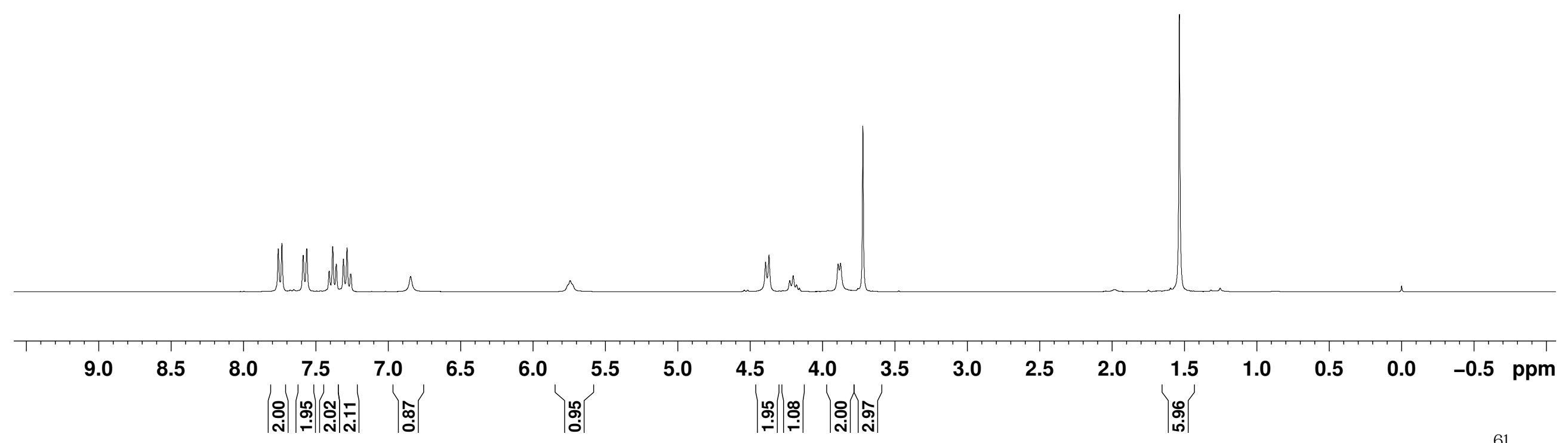




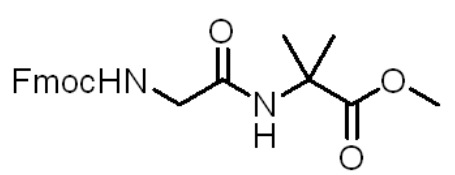

3ai, $75 \mathrm{MHz}, \mathrm{CDCl}_{3}$

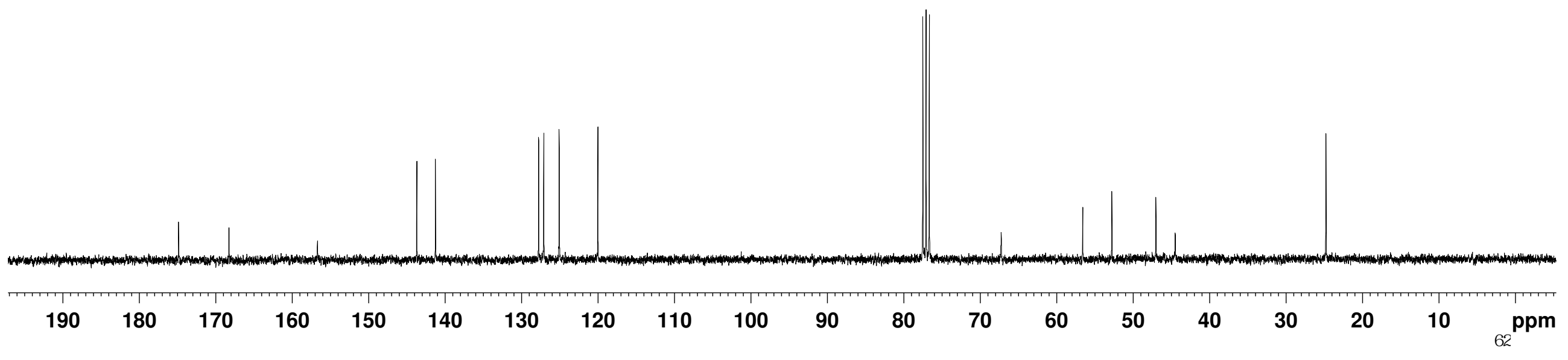




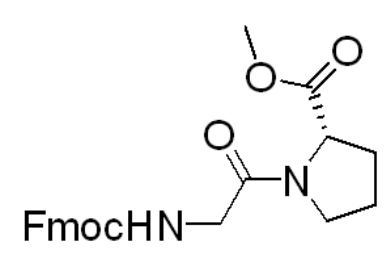

3aj, $300 \mathrm{MHz}, \mathrm{CDCl}_{3}$

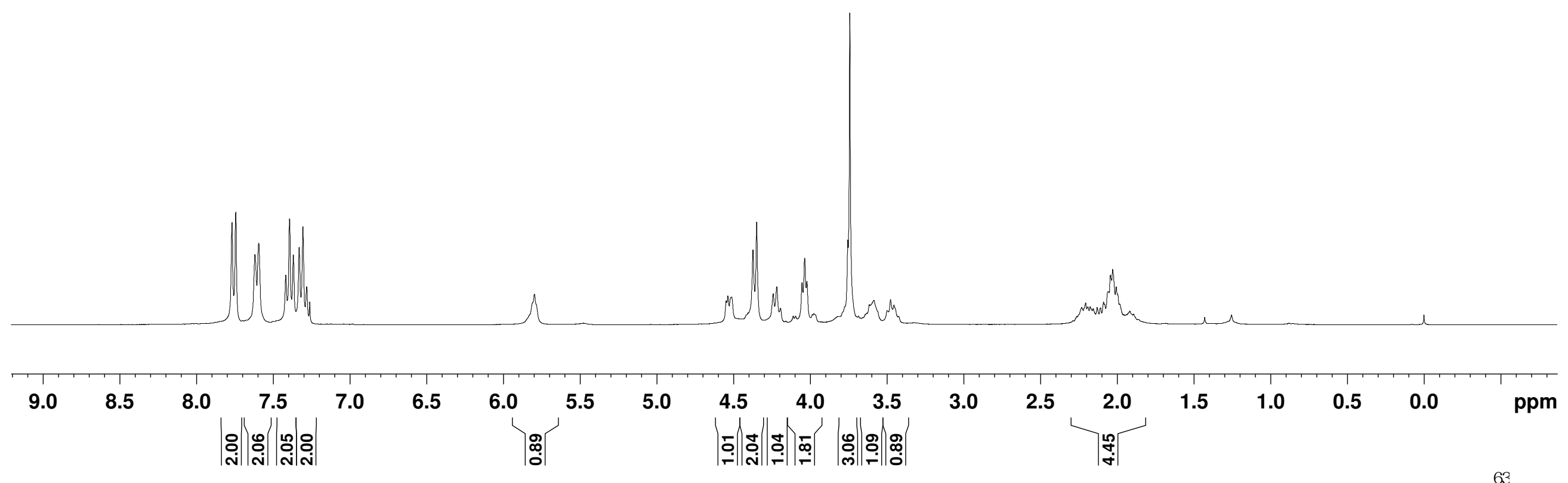




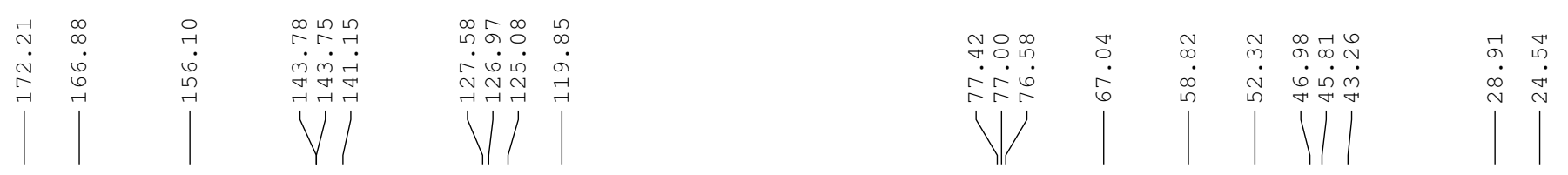

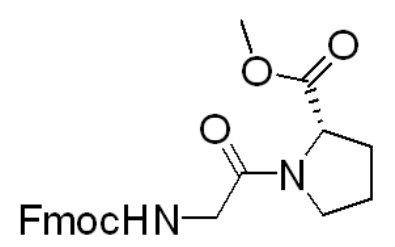

3aj, $75 \mathrm{MHz}, \mathrm{CDCl}_{3}$

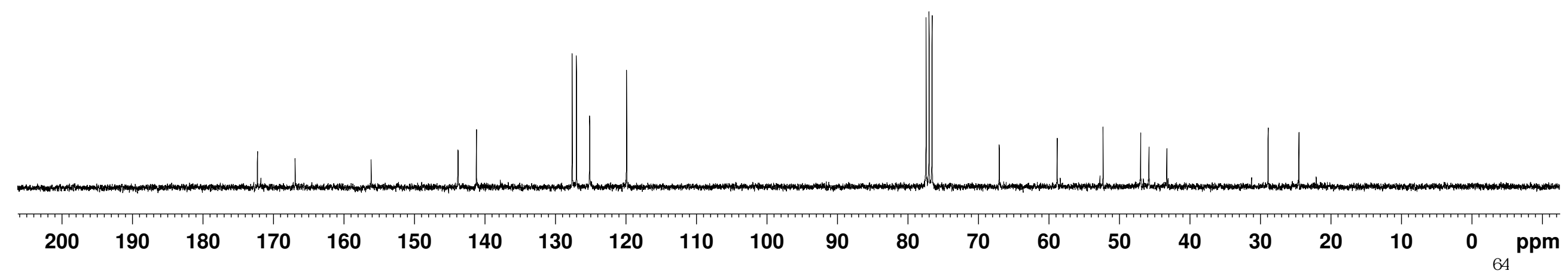



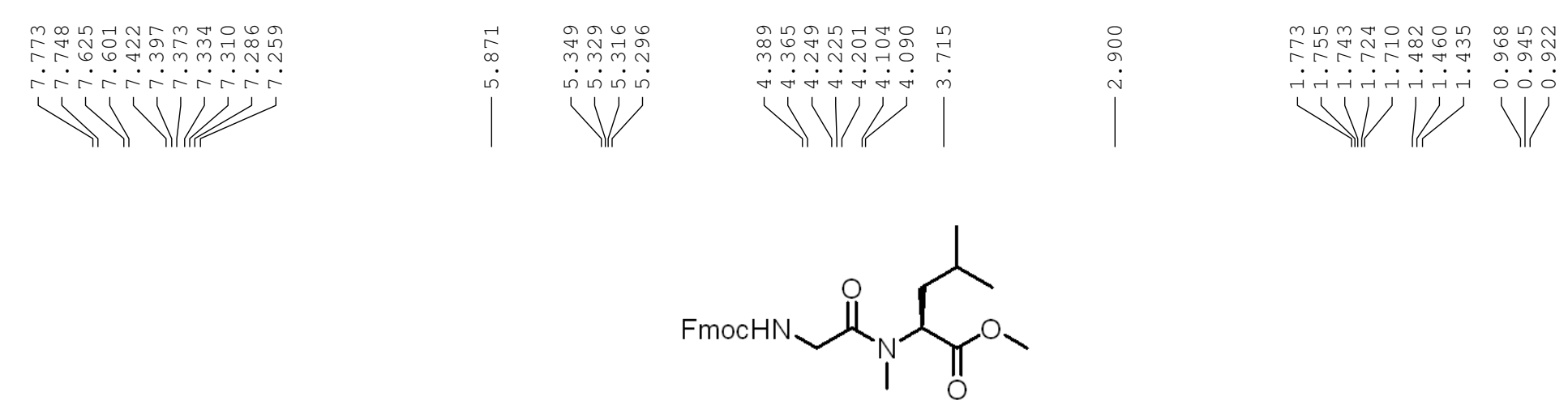

3ak, $300 \mathrm{MHz}, \mathrm{CDCl}_{3}$

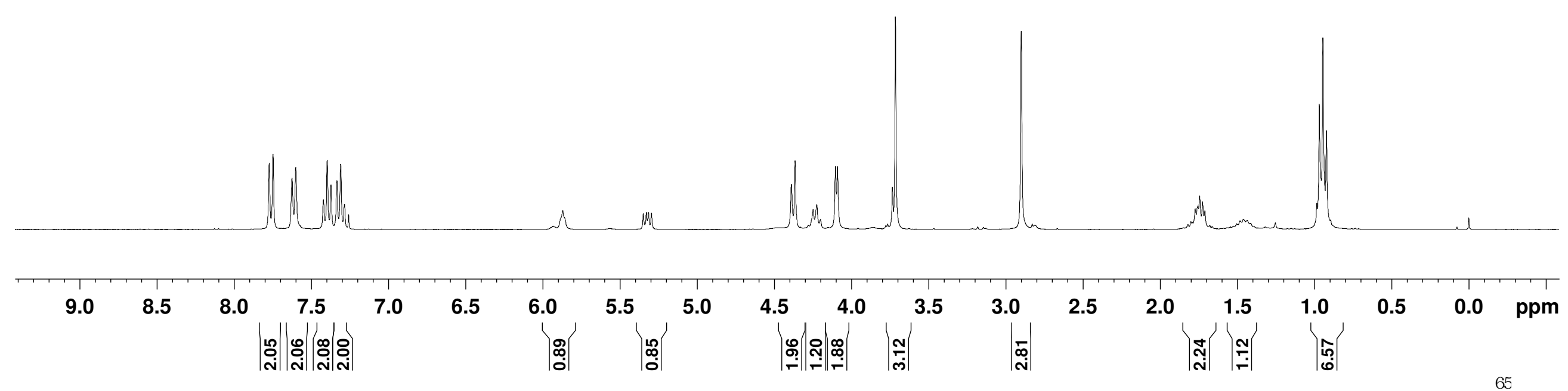




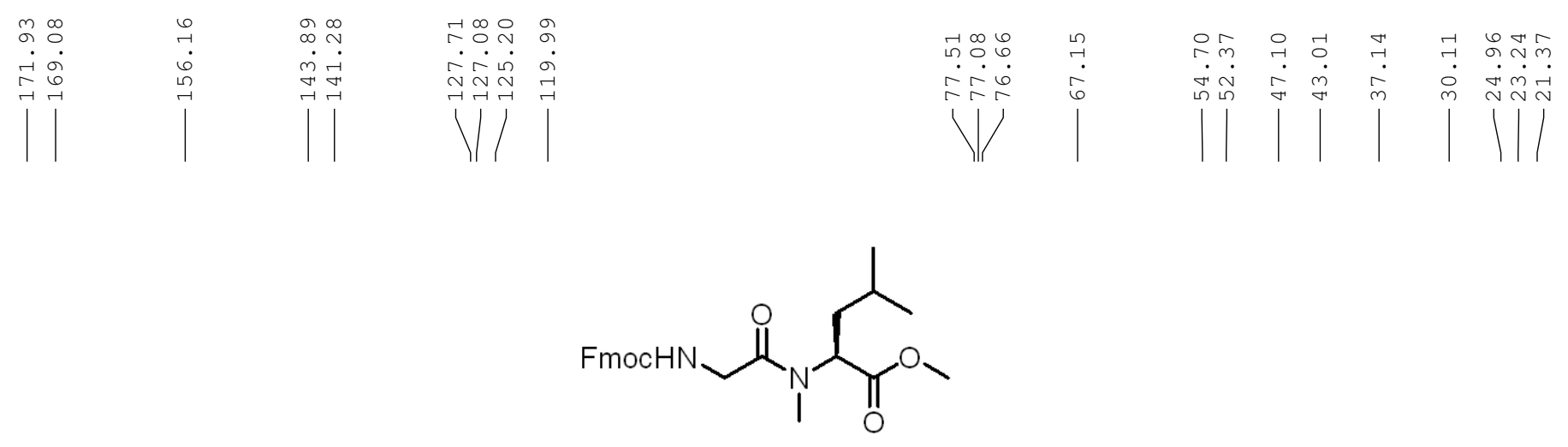

3ak, $75 \mathrm{MHz}, \mathrm{CDCl}_{3}$

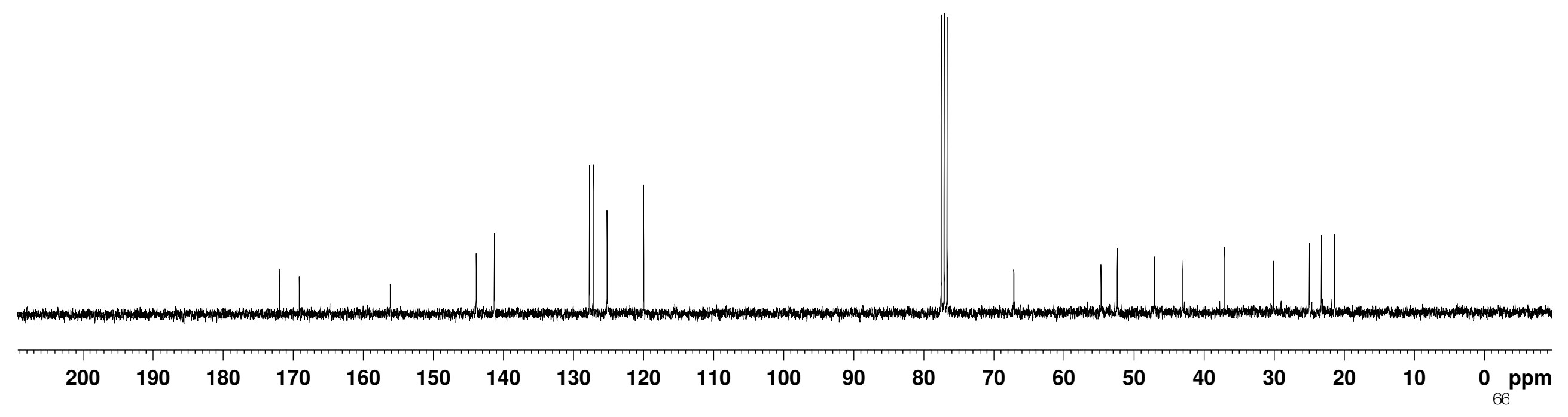



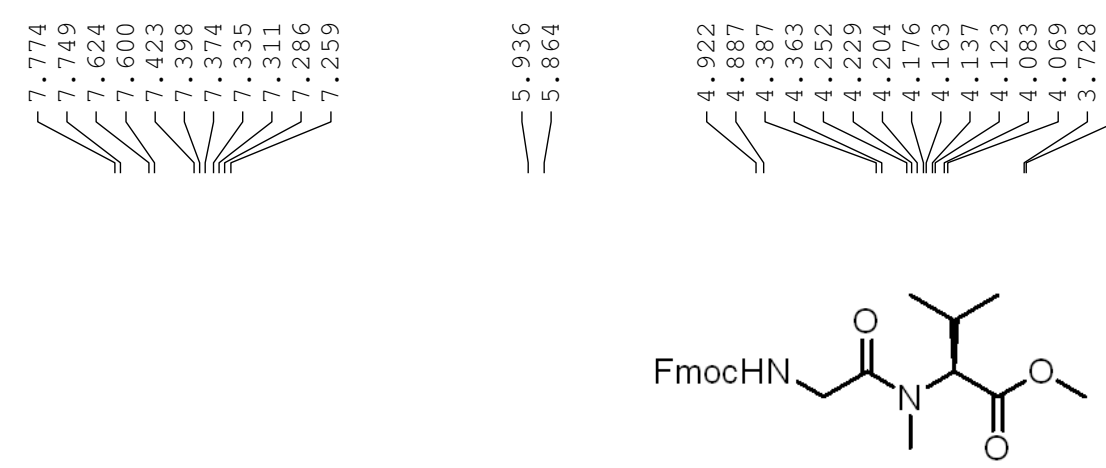

3al, $300 \mathrm{MHz}, \mathrm{CDCl}_{3}$

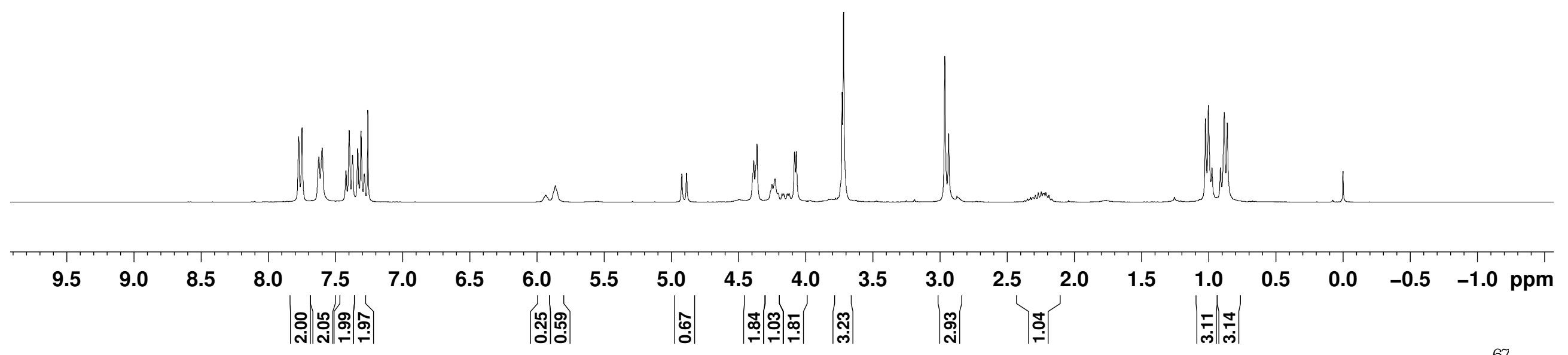




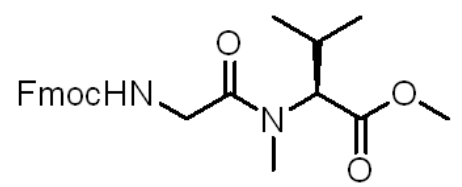

3al, $75 \mathrm{MHz}, \mathrm{CDCl}_{3}$

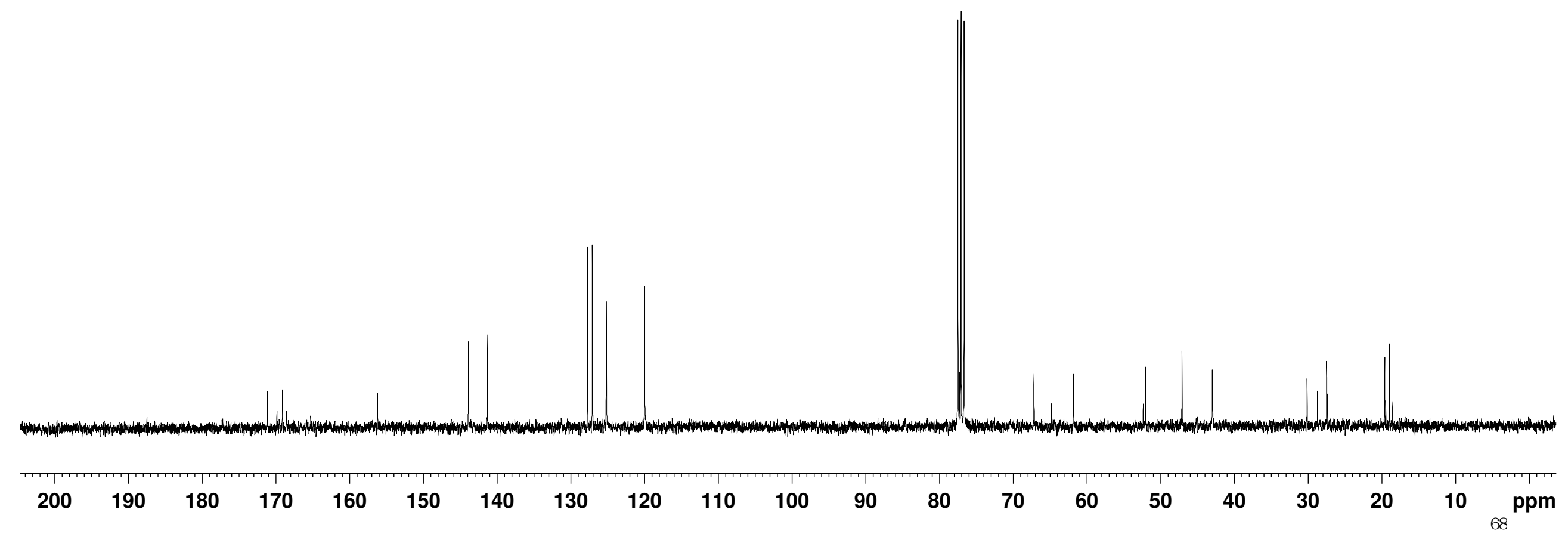




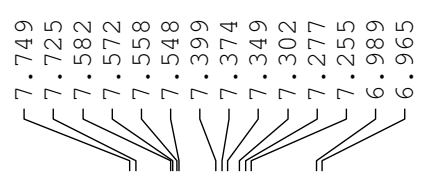

$\begin{array}{ll}r & 0 \\ 5 & 0 \\ \infty & \infty \\ 0 & 0 \\ n & 0\end{array}$
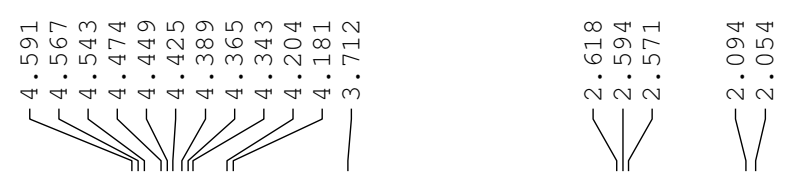

mo

$\circ$
$\circ$
0
1
1<smiles>COC(=O)[C@H](C)NC(=O)[C@H](CCSC)NC=O</smiles>

3ba, $300 \mathrm{MHz}, \mathrm{CDCl}_{3}$

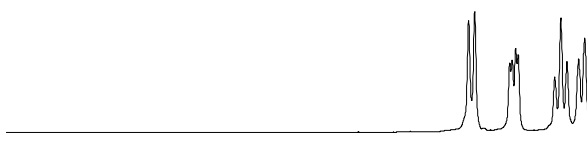

Mund
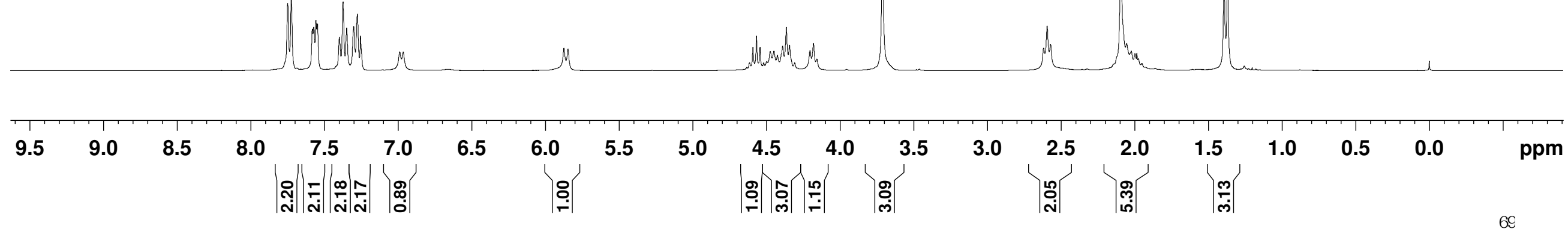


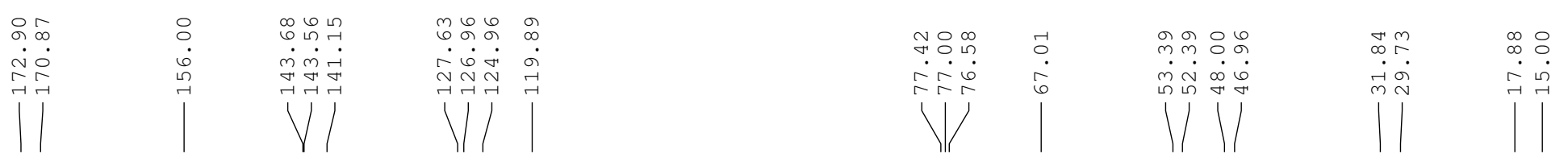

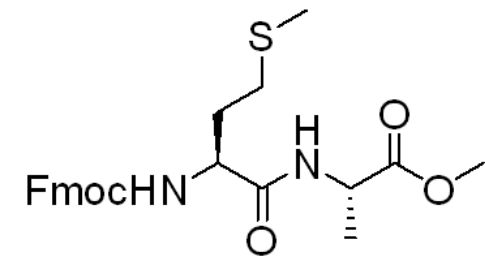

3ba, $75 \mathrm{MHz}, \mathrm{CDCl}_{3}$

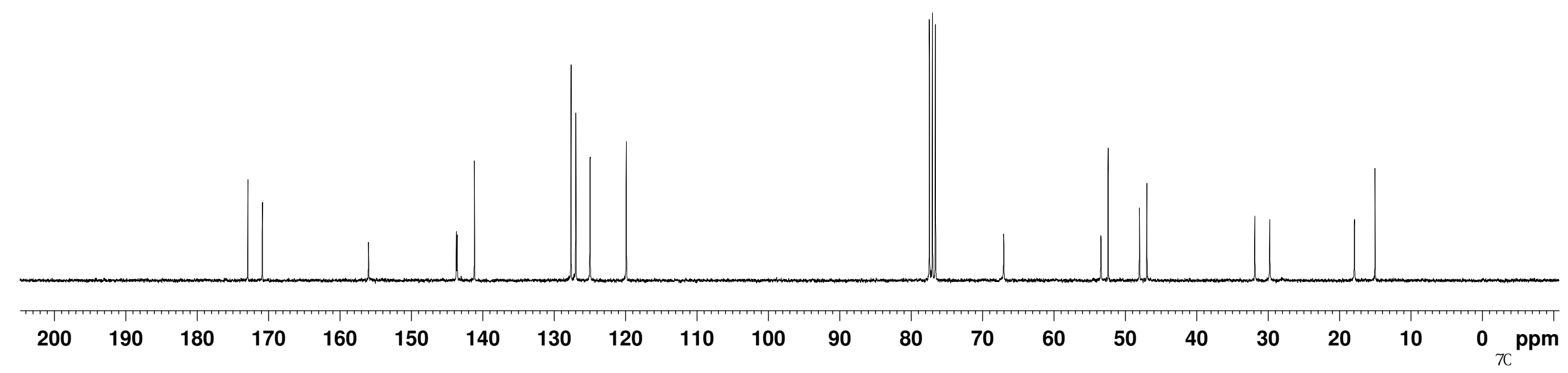




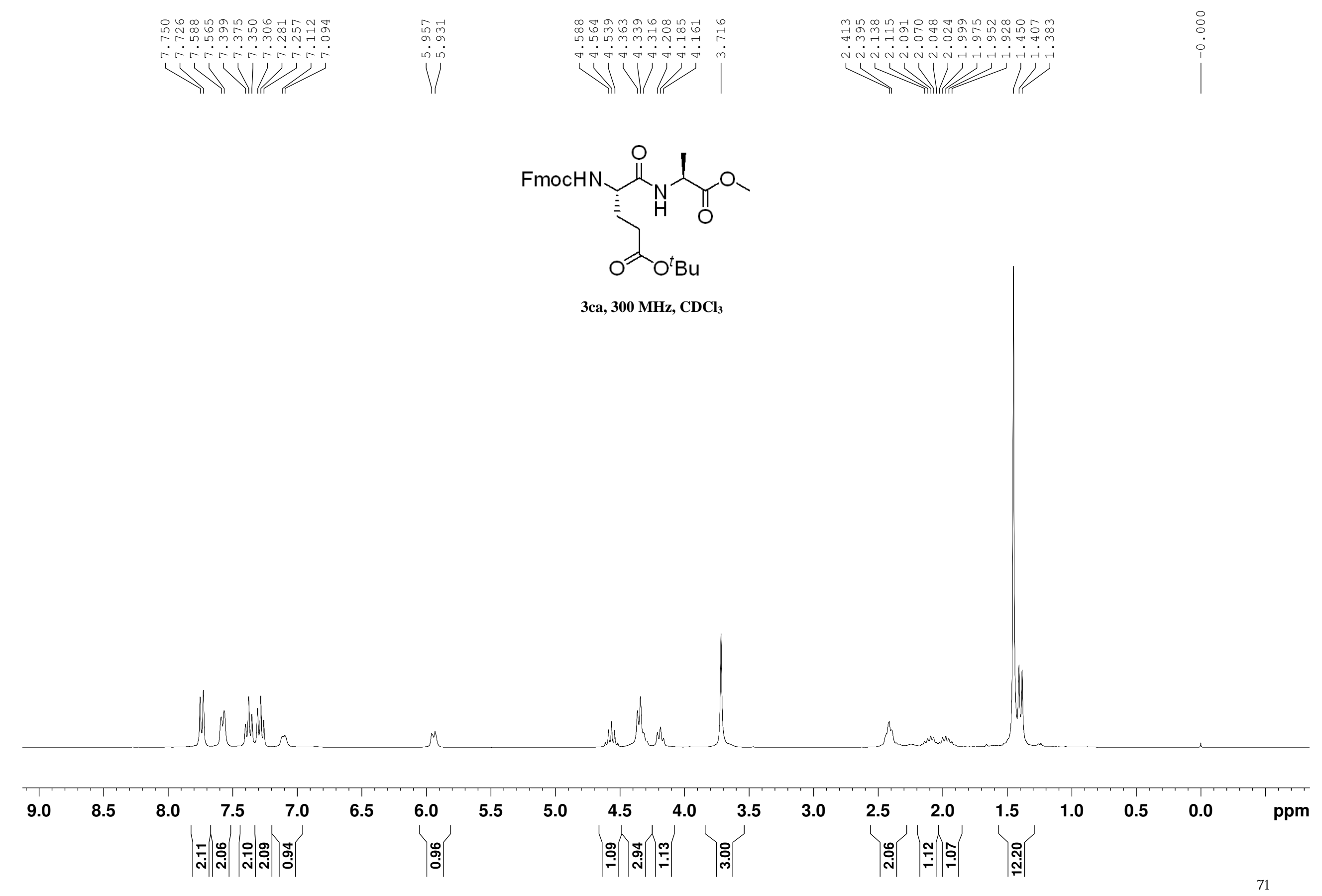




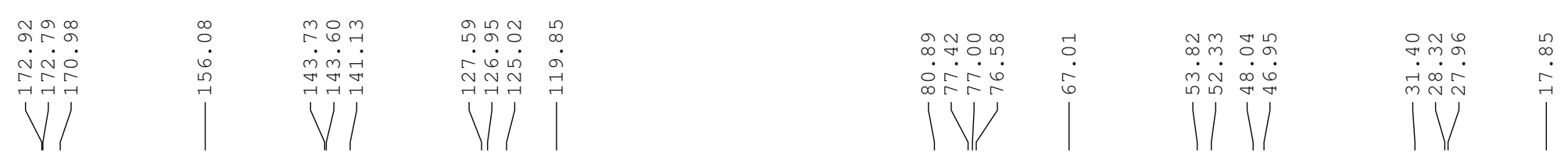

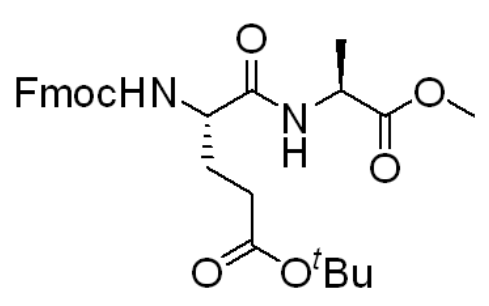

3ca, $75 \mathrm{MHz}, \mathrm{CDCl}_{3}$

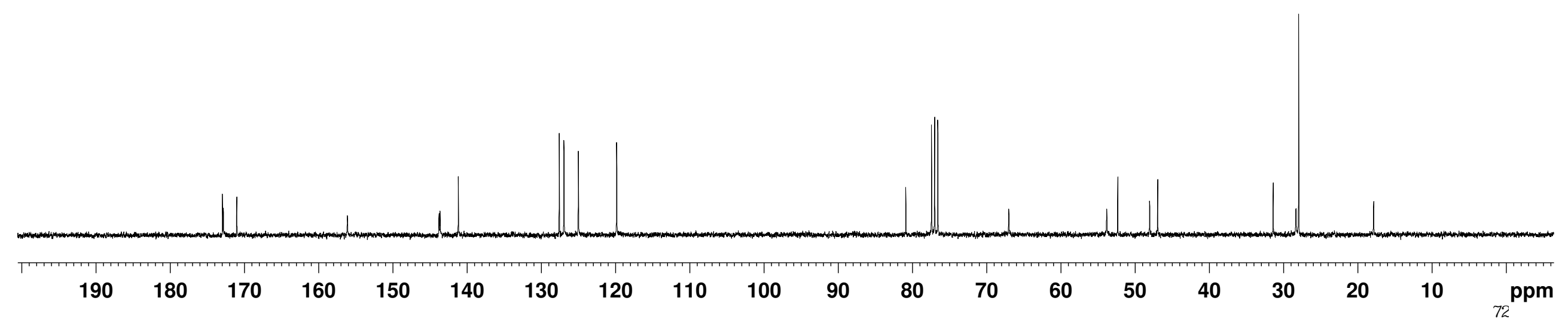




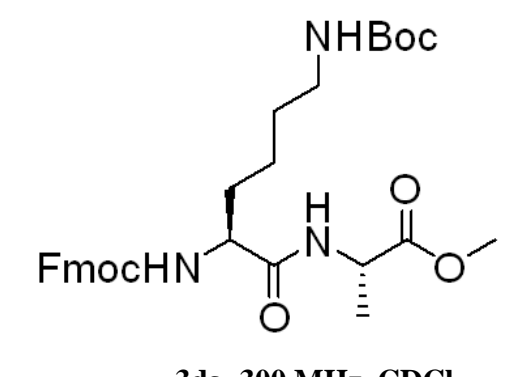

3da, $300 \mathrm{MHz}, \mathrm{CDCl}_{3}$

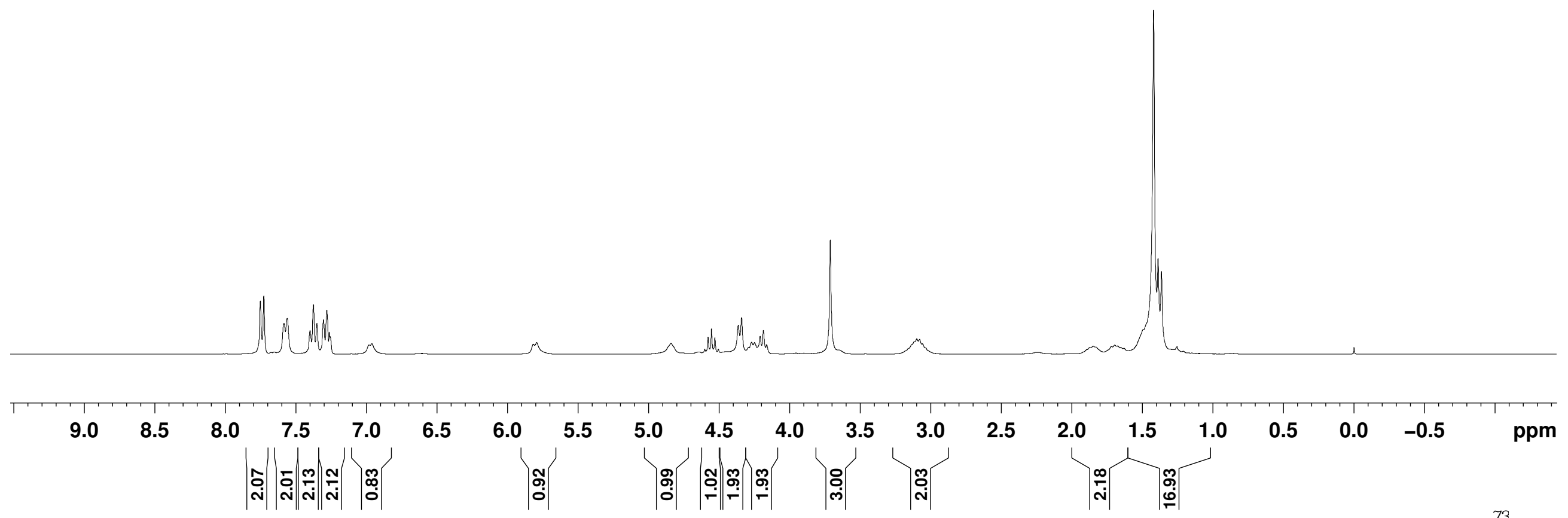




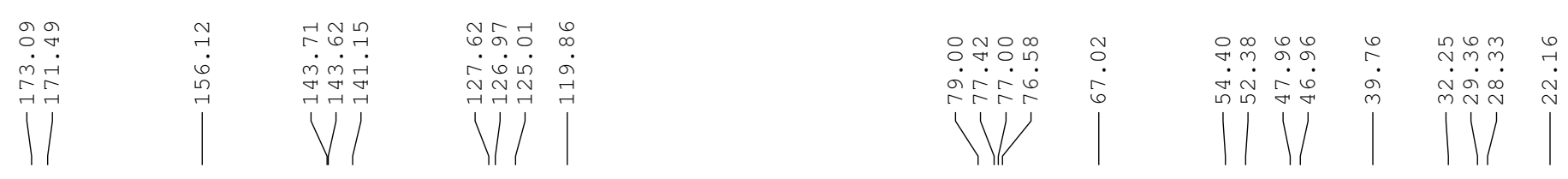

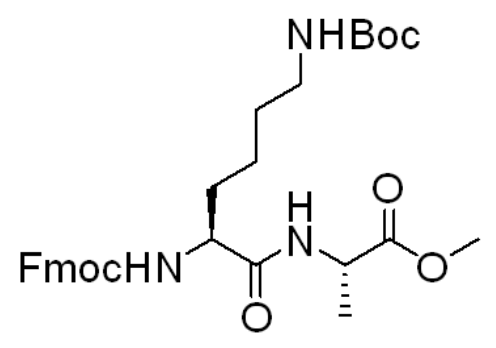

3da, $75 \mathrm{MHz}, \mathrm{CDCl}_{3}$

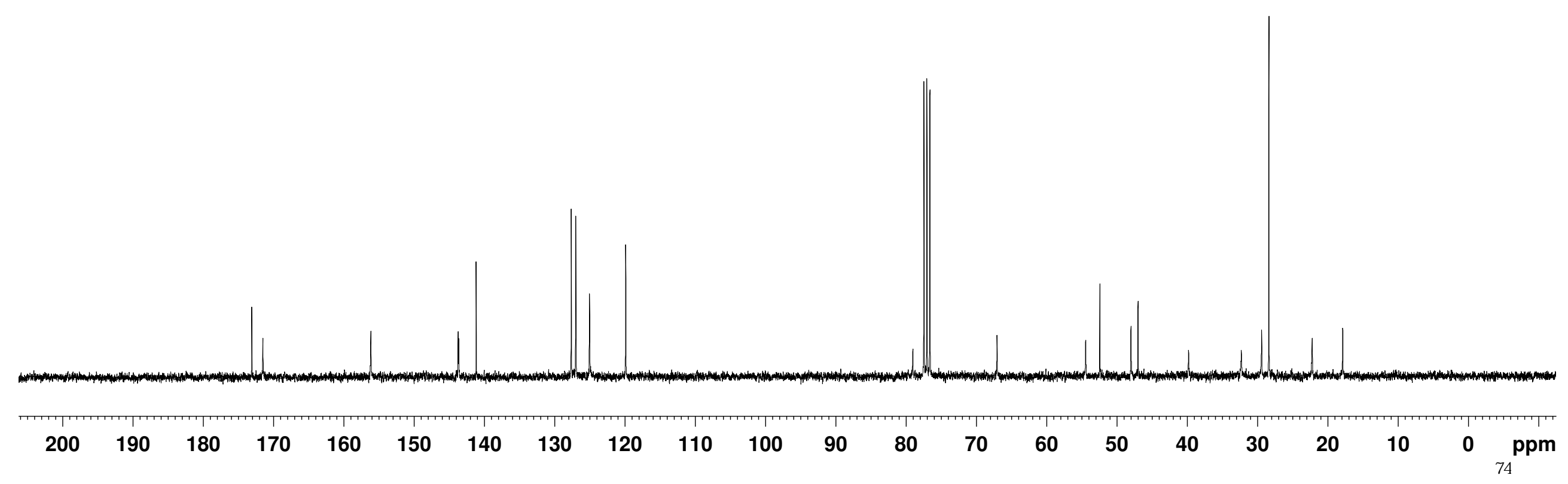



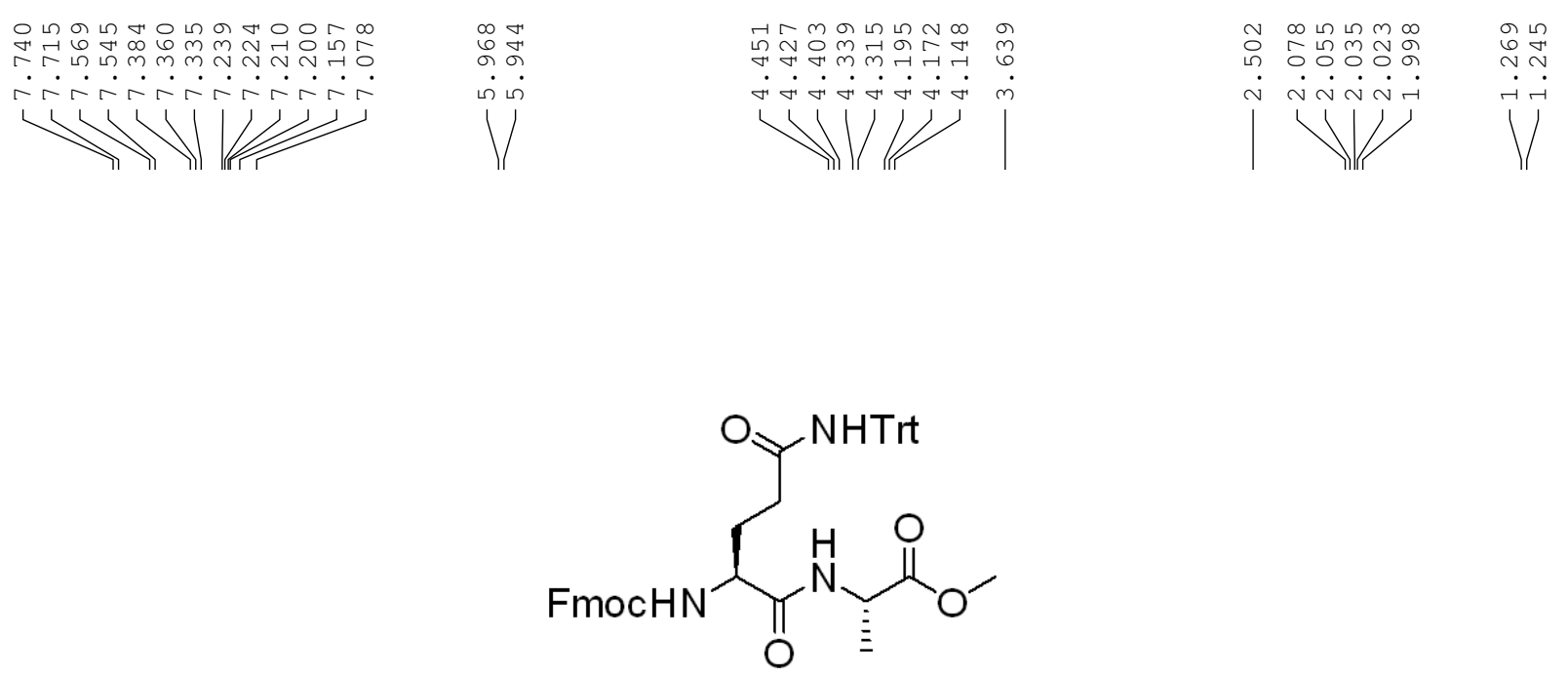

3ea, $300 \mathrm{MHz}, \mathrm{CDCl}_{3}$

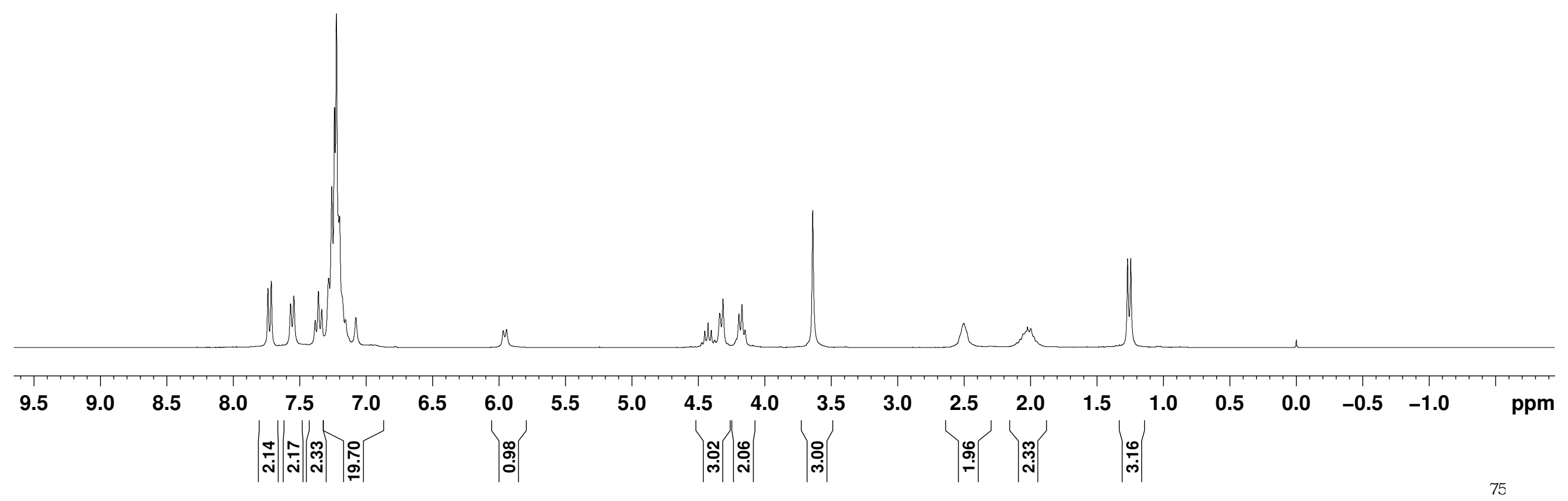




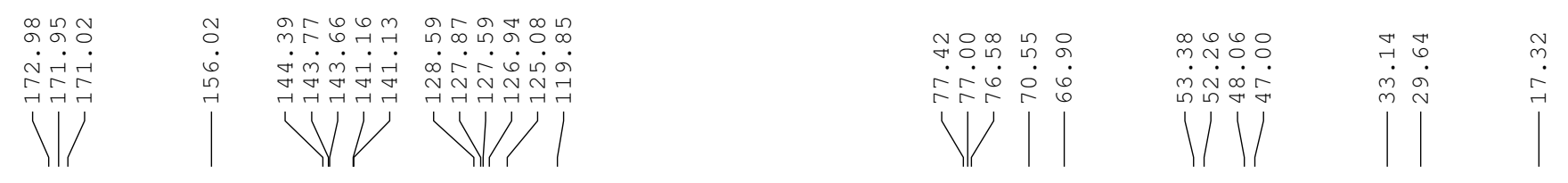

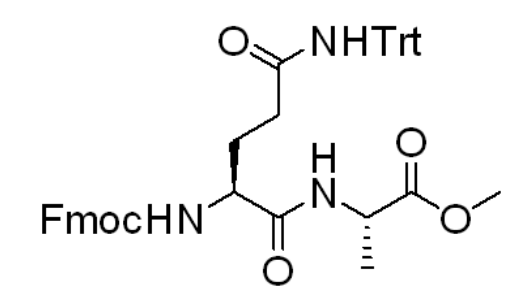

3ea, $75 \mathrm{MHz}, \mathrm{CDCl}_{3}$

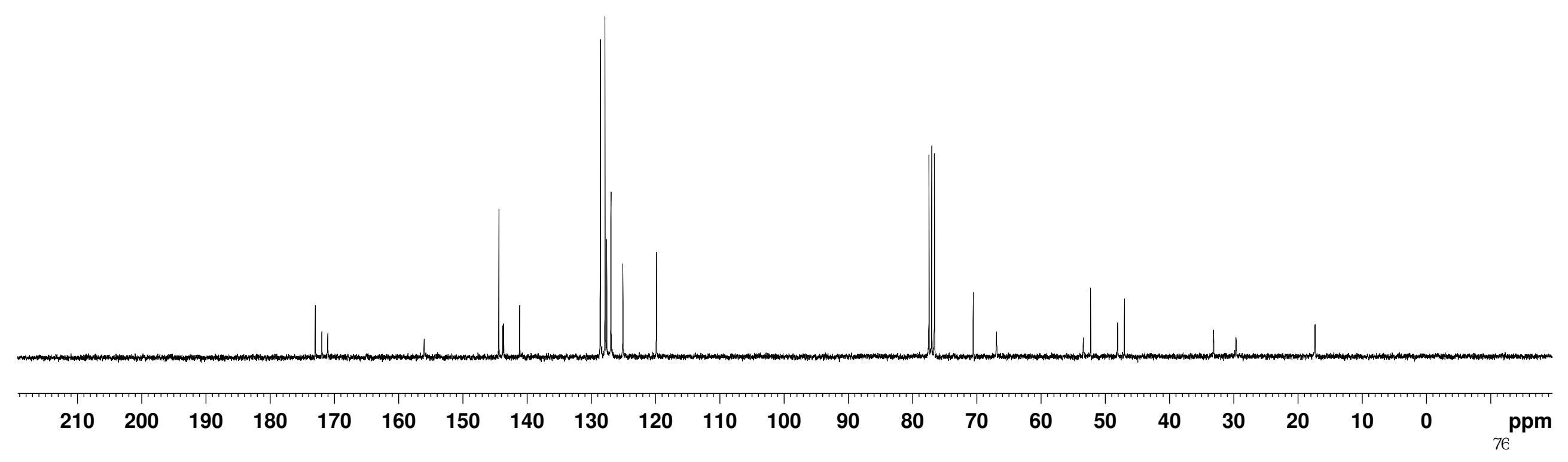




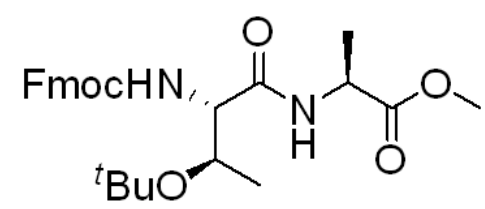

3fa, $300 \mathrm{MHz}, \mathrm{CDCl}_{3}$

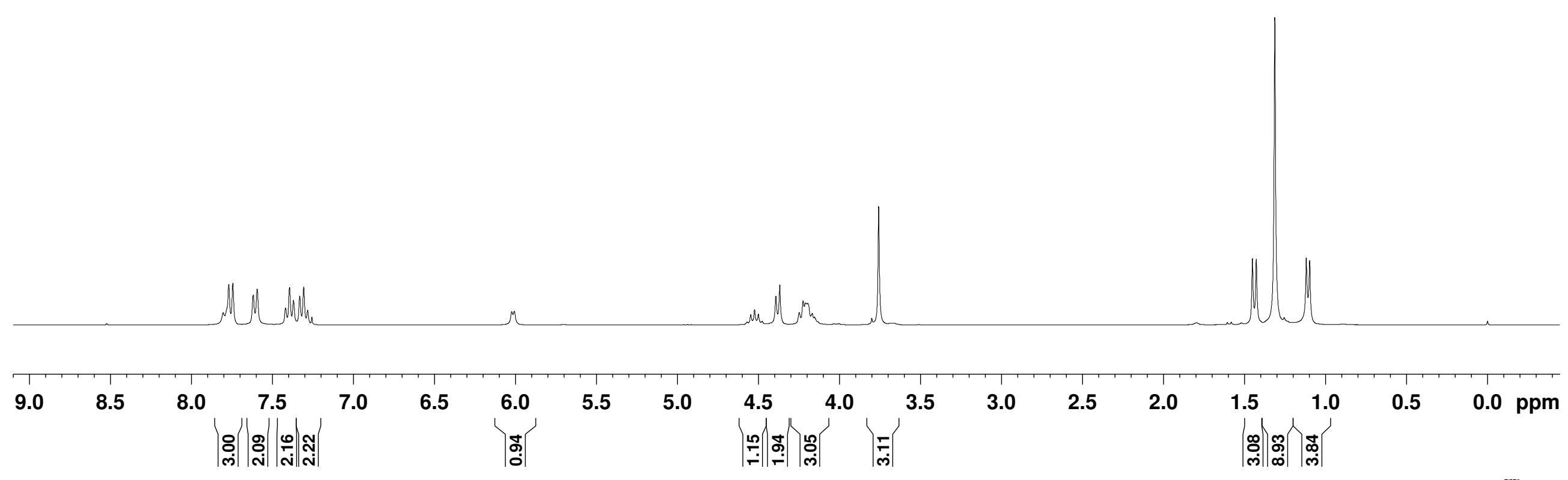




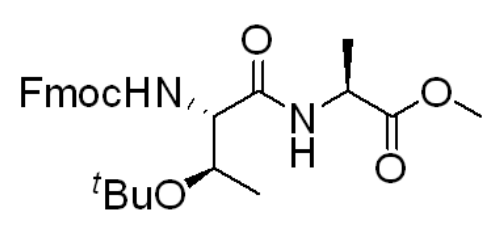

3fa, $75 \mathrm{MHz}, \mathrm{CDCl}_{3}$

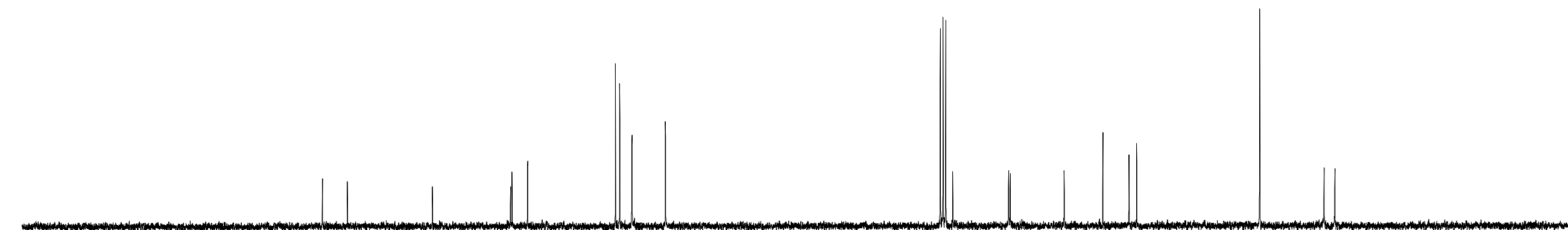




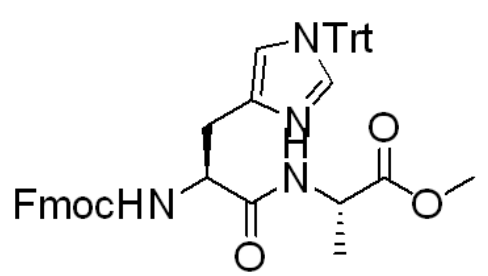

3ga, $300 \mathrm{MHz}, \mathrm{CDCl}_{3}$

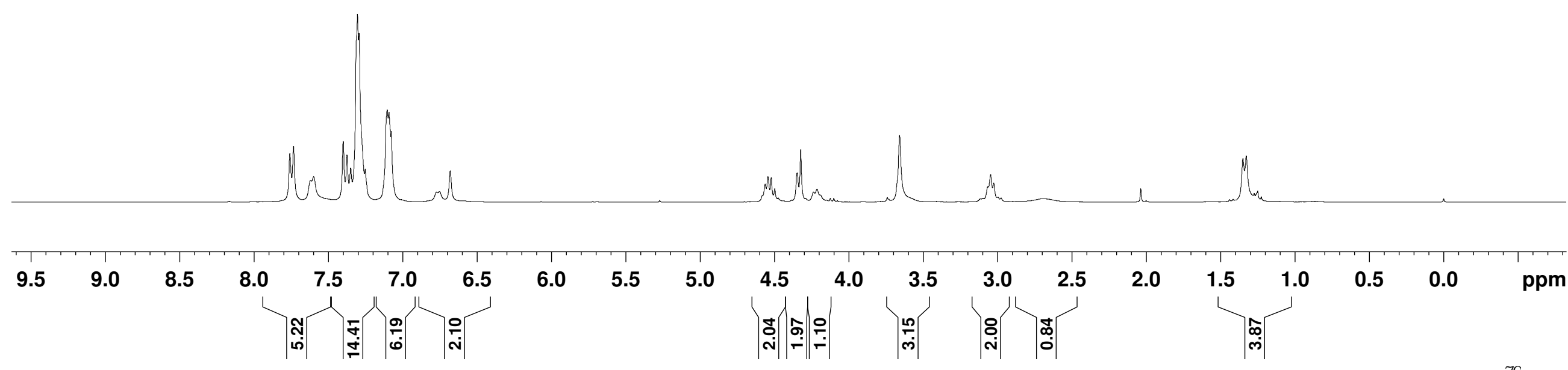




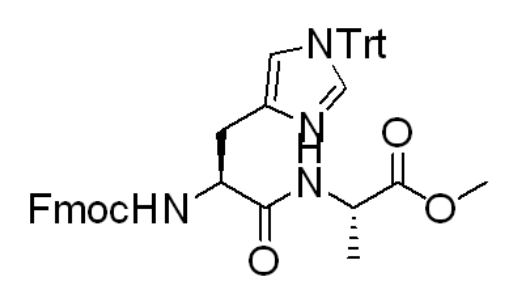

3ga, $75 \mathrm{MHz}, \mathrm{CDCl}_{3}$

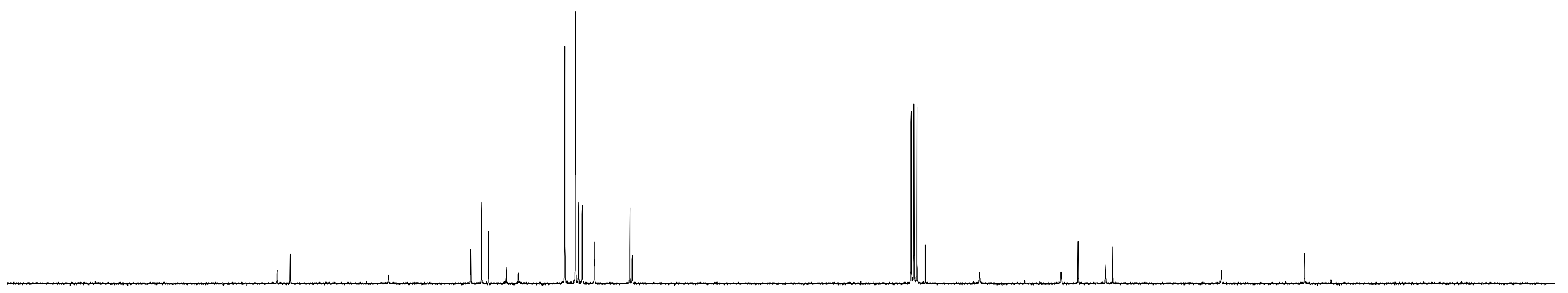




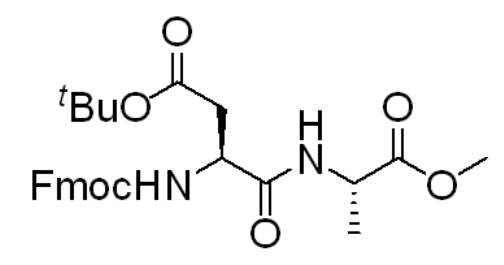

3ha, $300 \mathrm{MHz}, \mathrm{CDCl}_{3}$

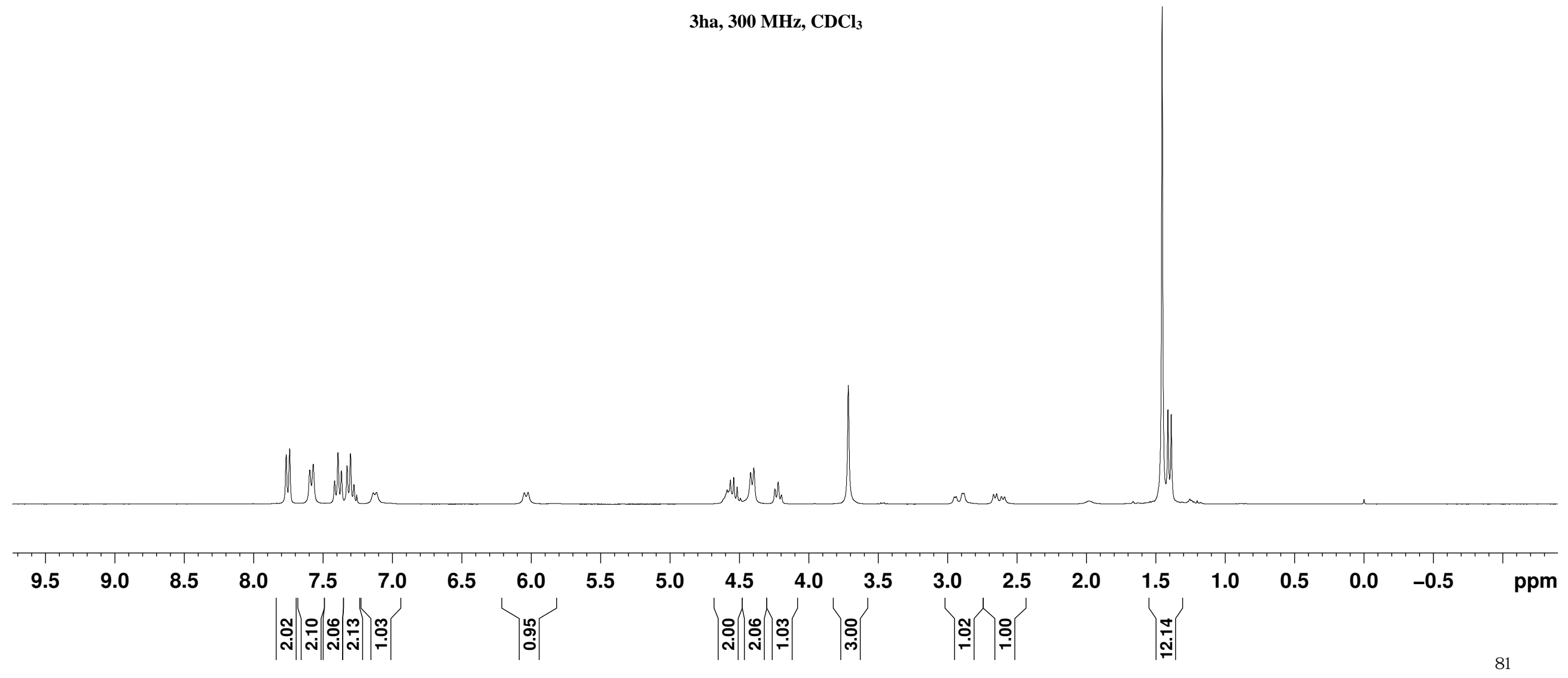




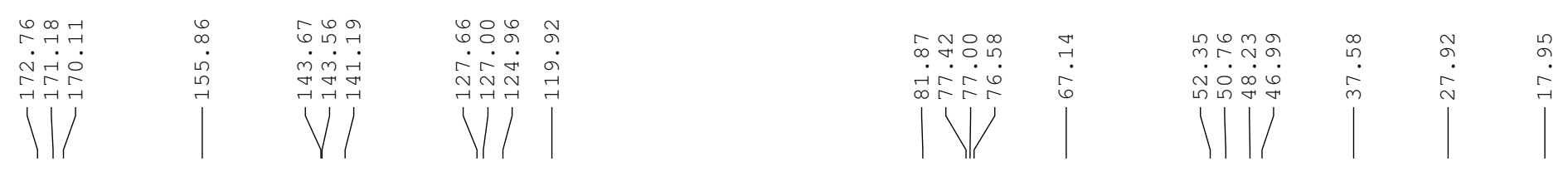

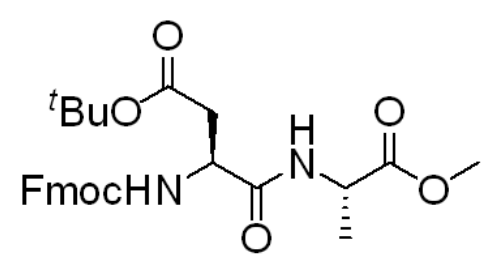

3ha, $75 \mathrm{MHz}, \mathrm{CDCl}_{3}$

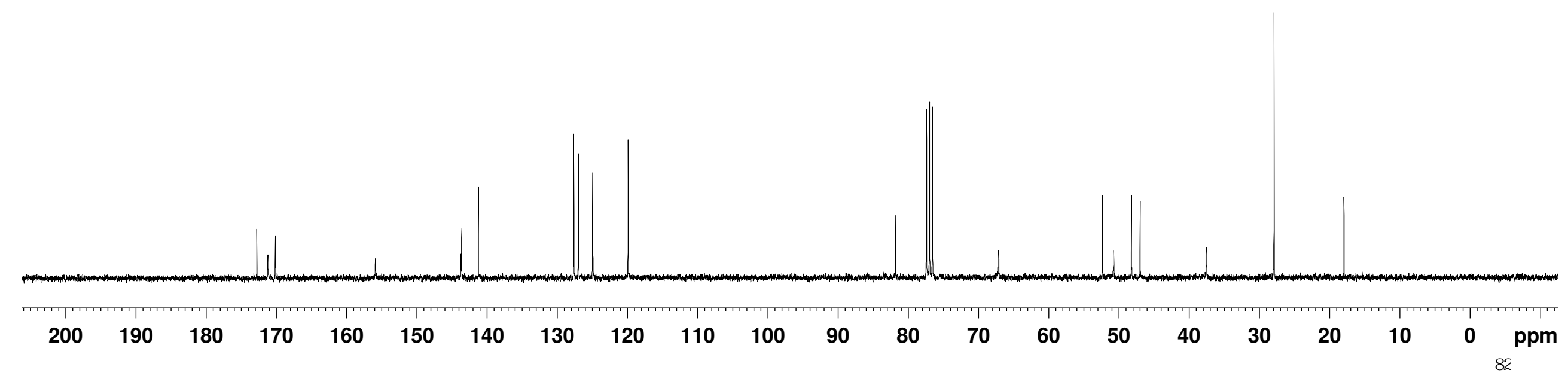




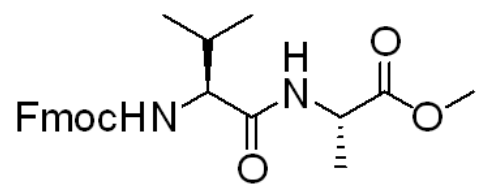

3ia, $300 \mathrm{MHz}, \mathrm{CDCl}_{3}$

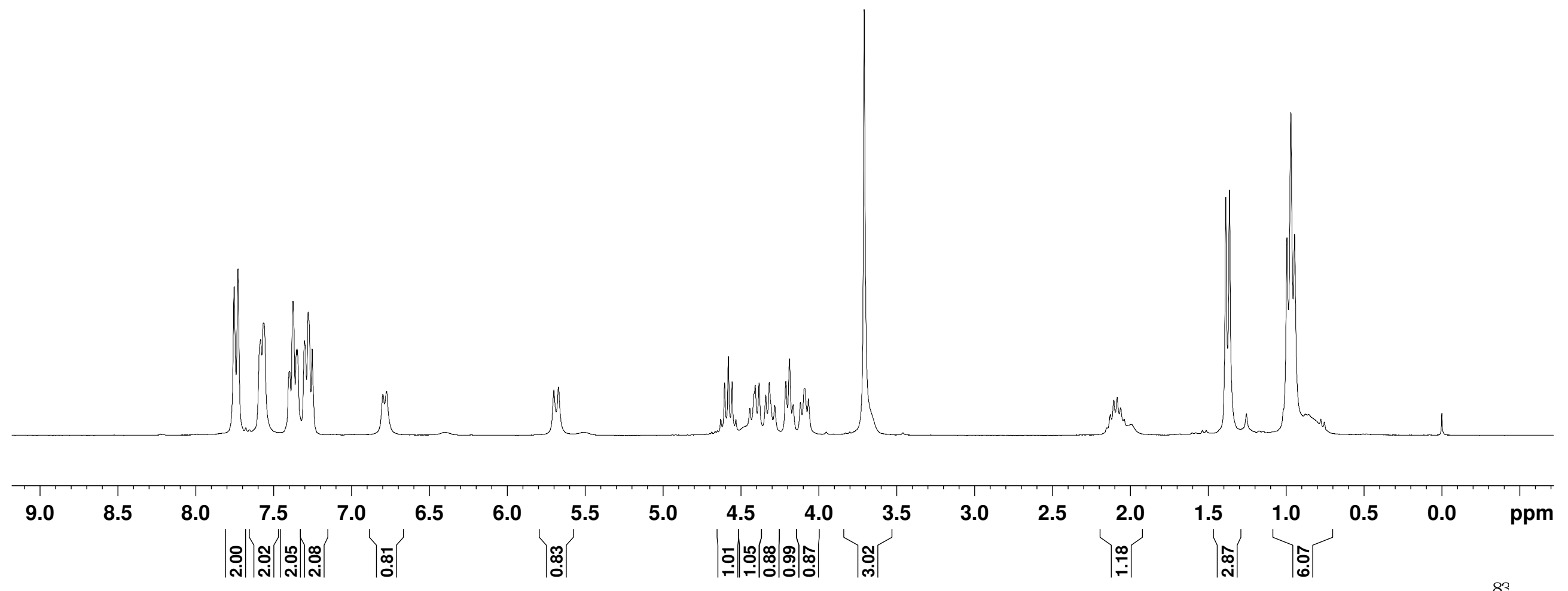




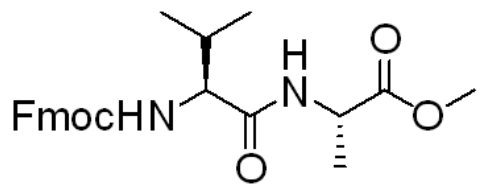

3ia, 75 MHz, CDCl

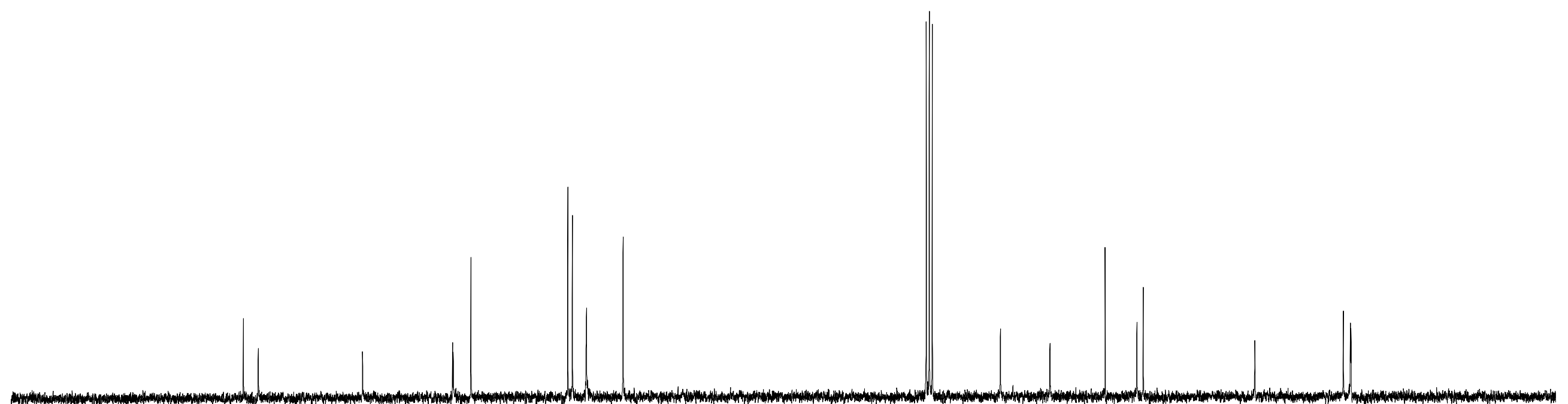




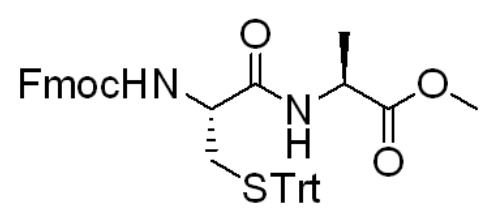

3ja, $300 \mathrm{MHz}, \mathrm{CDCl}_{3}$

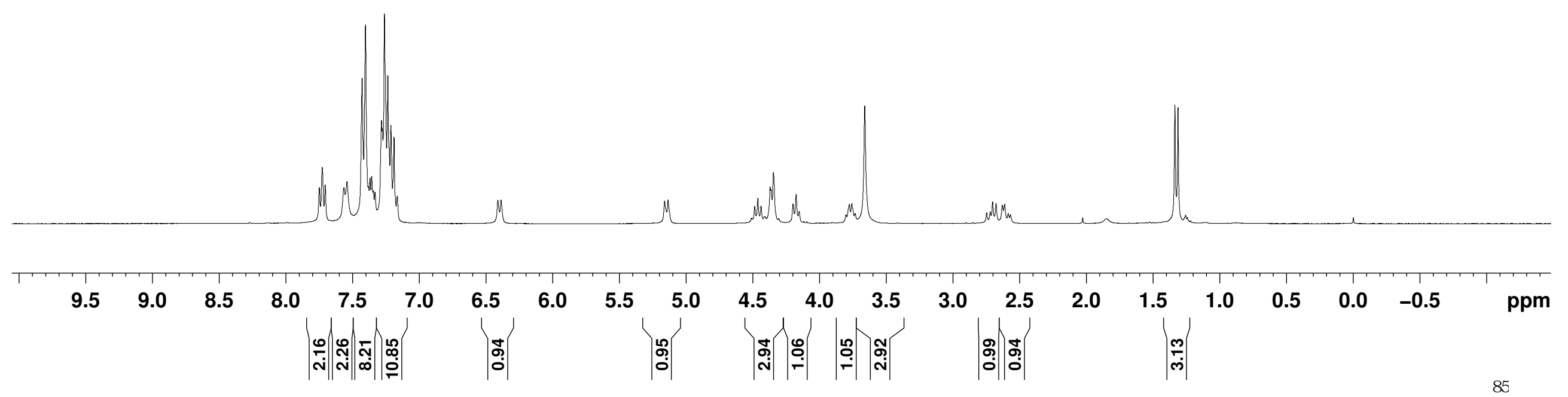




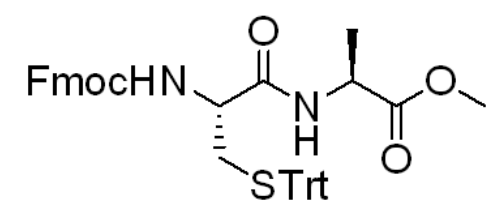

3ja, $75 \mathrm{MHz}, \mathrm{CDCl}_{3}$

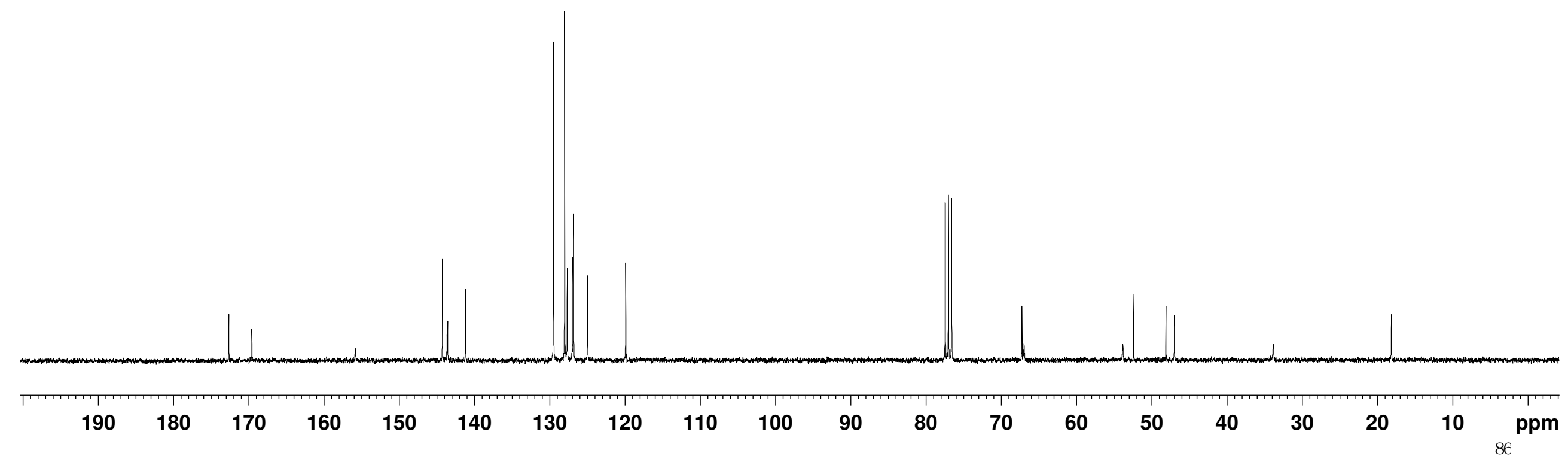




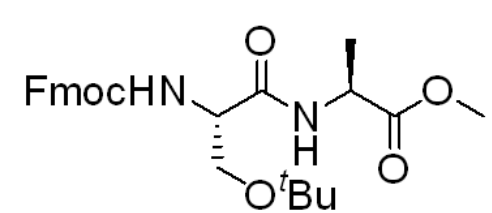

3ka, $300 \mathrm{MHz}, \mathrm{CDCl}_{3}$

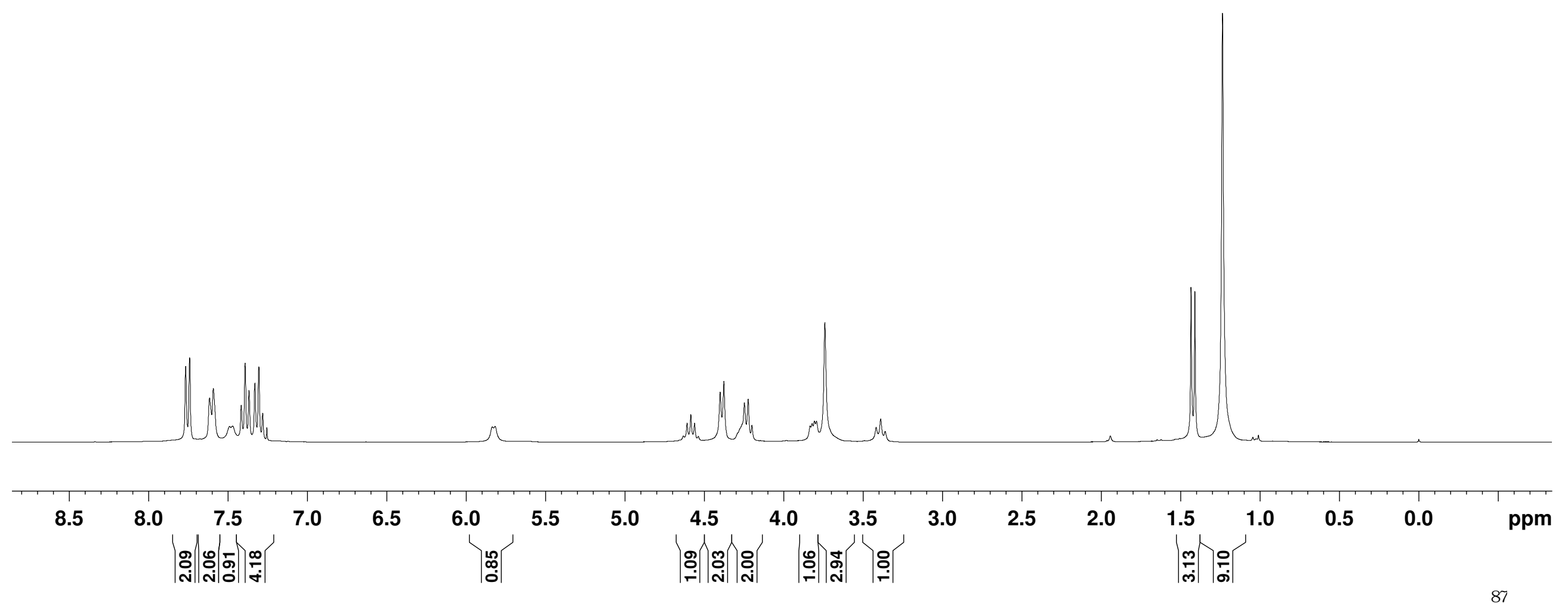




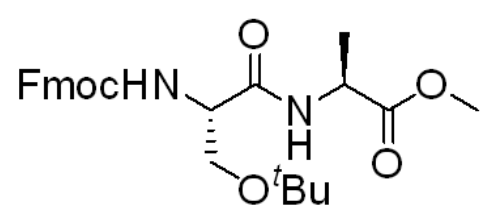

3ka, 75 MHz, $\mathrm{CDCl}_{3}$

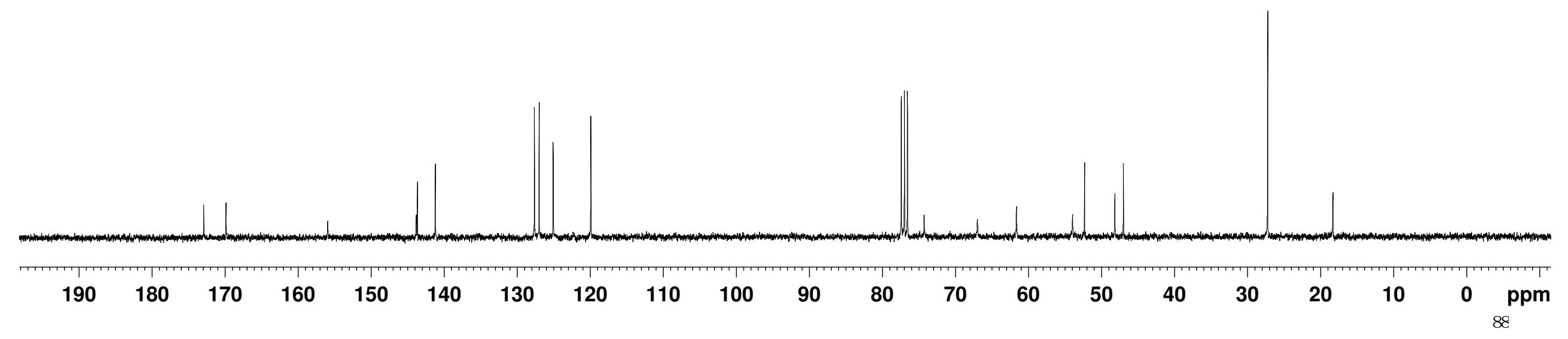




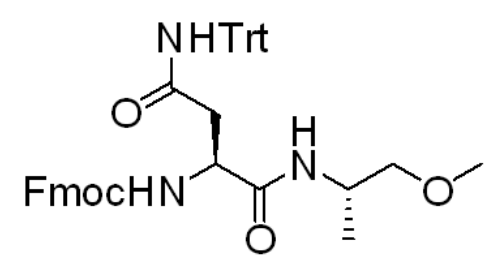

3la, $300 \mathrm{MHz}, \mathrm{CDCl}_{3}$

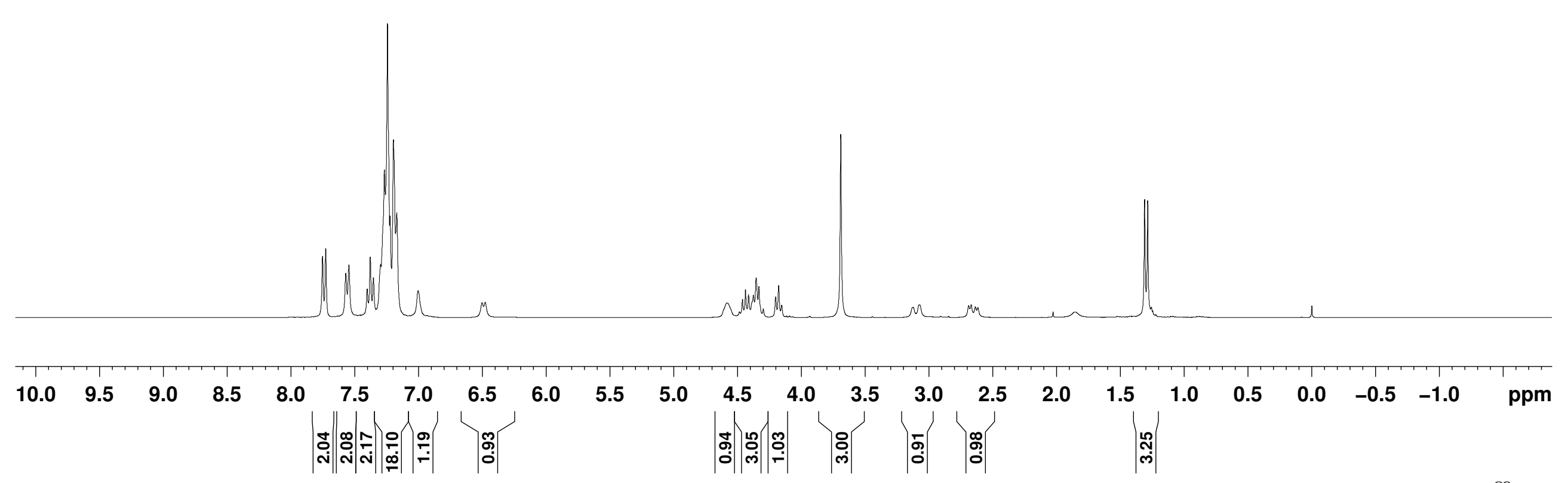




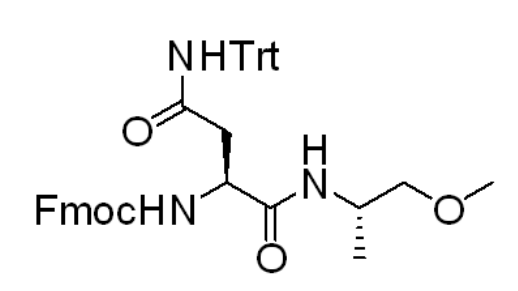

3la, $75 \mathrm{MHz}, \mathrm{CDCl}_{3}$

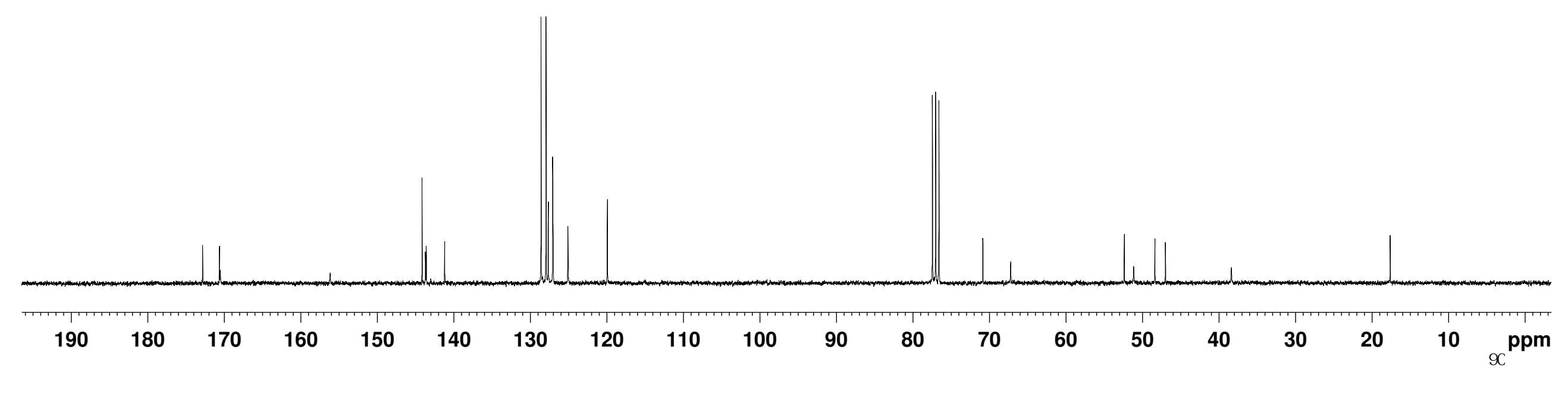




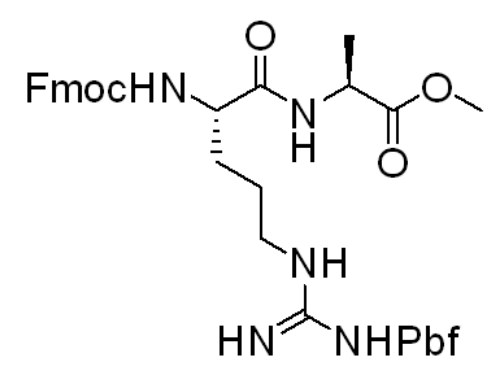

3ma, $300 \mathrm{MHz}, \mathrm{CDCl}_{3}$

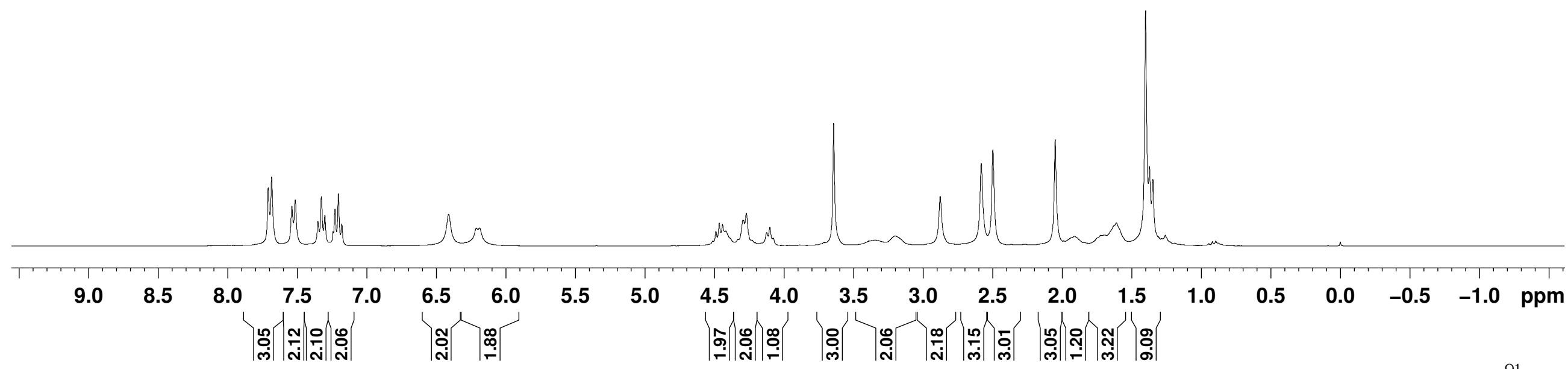




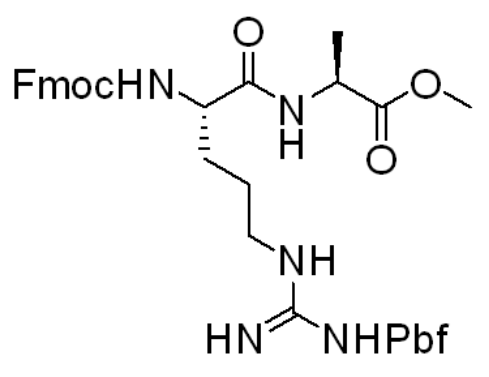

3ma, $75 \mathrm{MHz}, \mathrm{CDCl}_{3}$

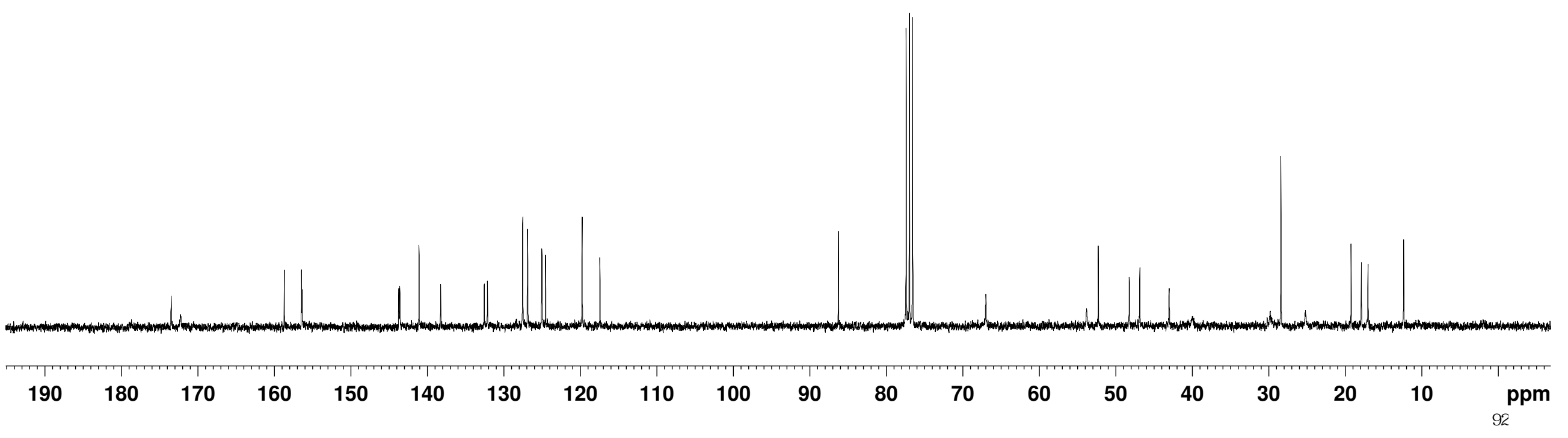



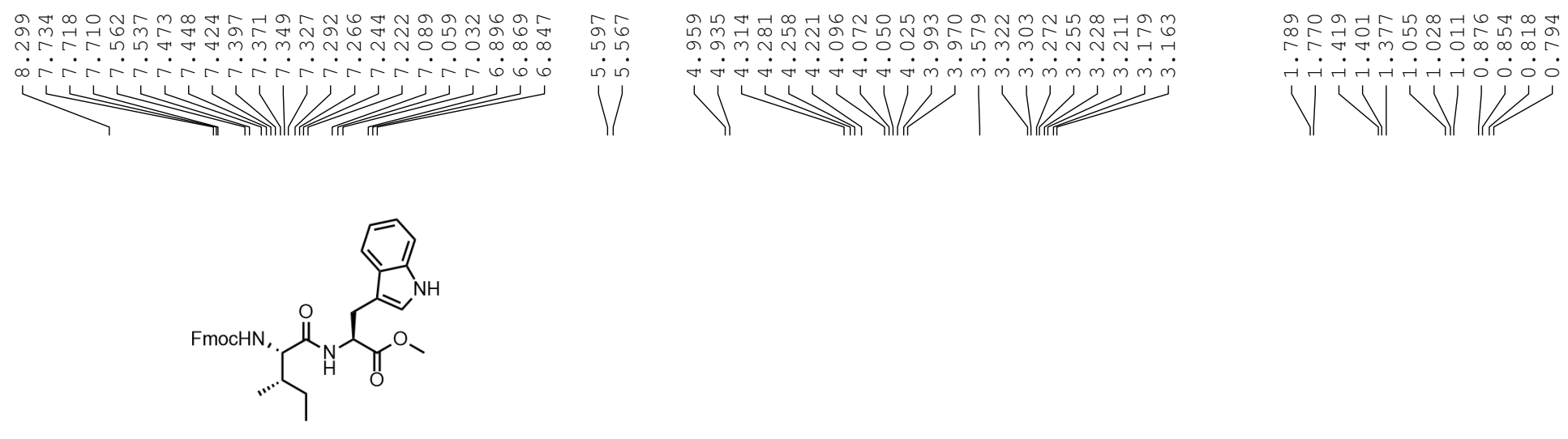

3na, $300 \mathrm{MHz}, \mathrm{CDCl}_{3}$

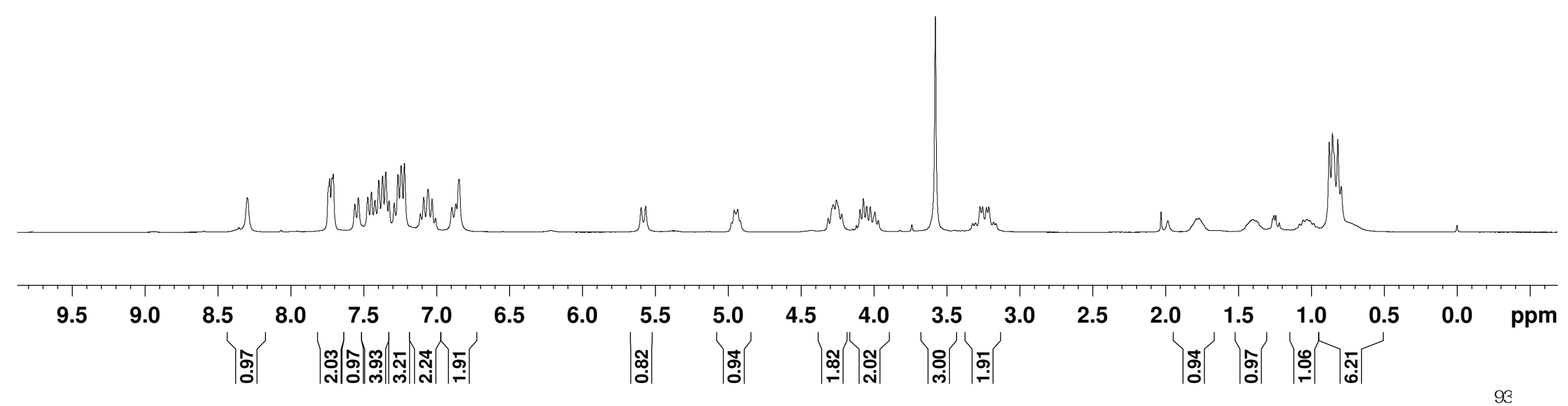




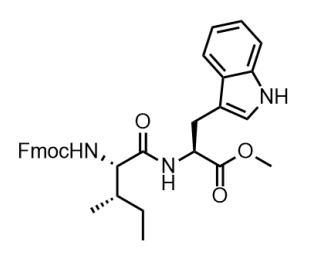

3na, $75 \mathrm{MHz}, \mathrm{CDCl}_{3}$

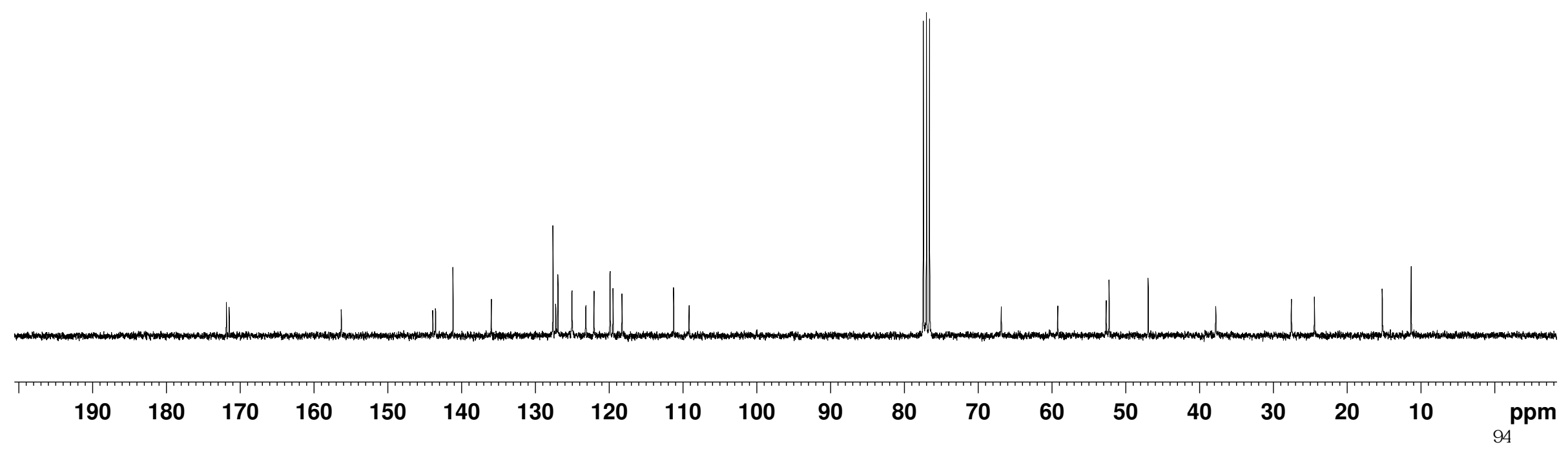




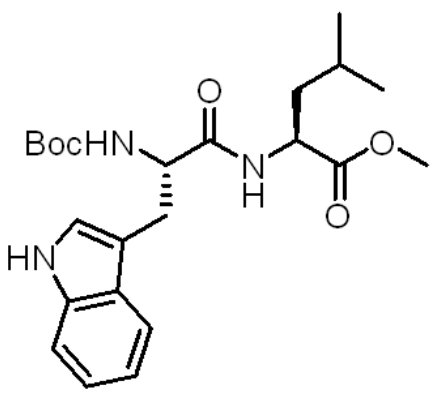

3oa, $300 \mathrm{MHz}, \mathrm{CDCl}_{3}$

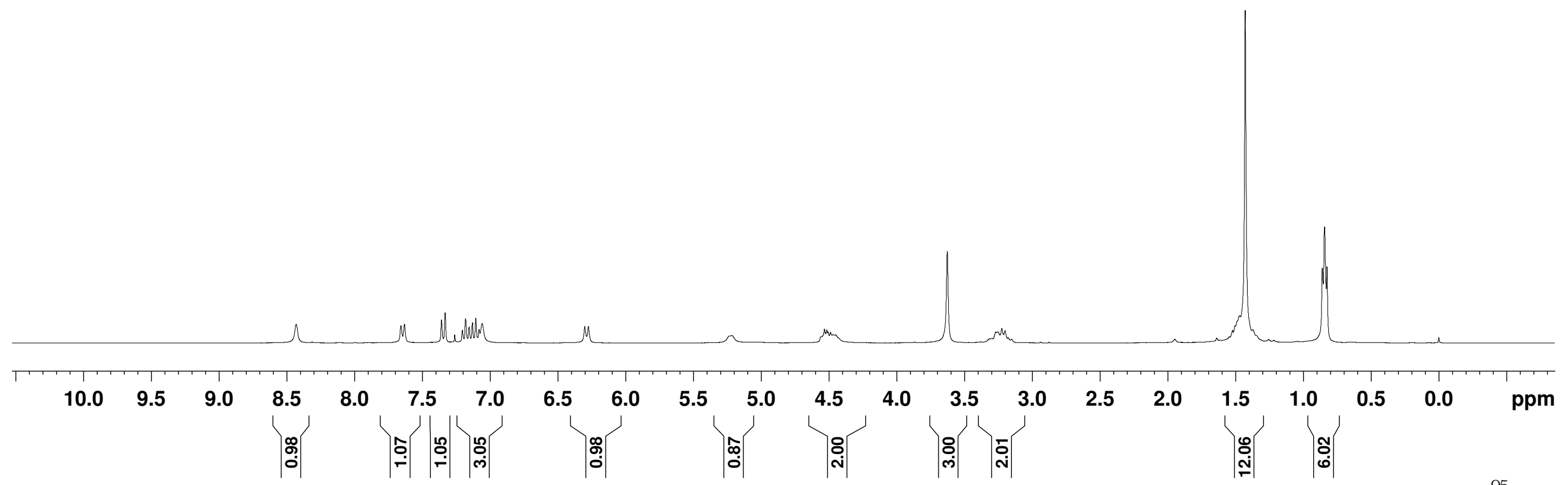




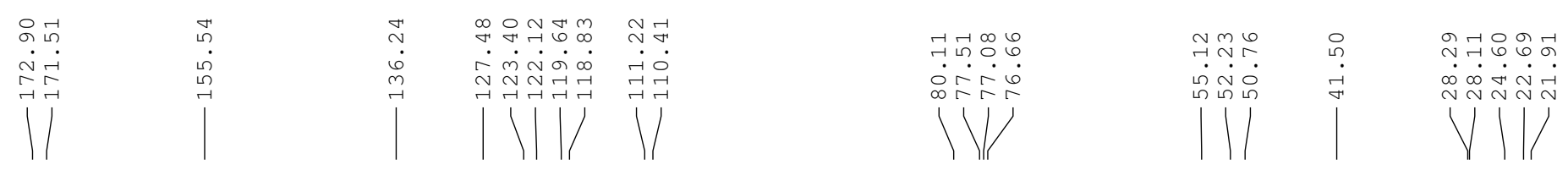
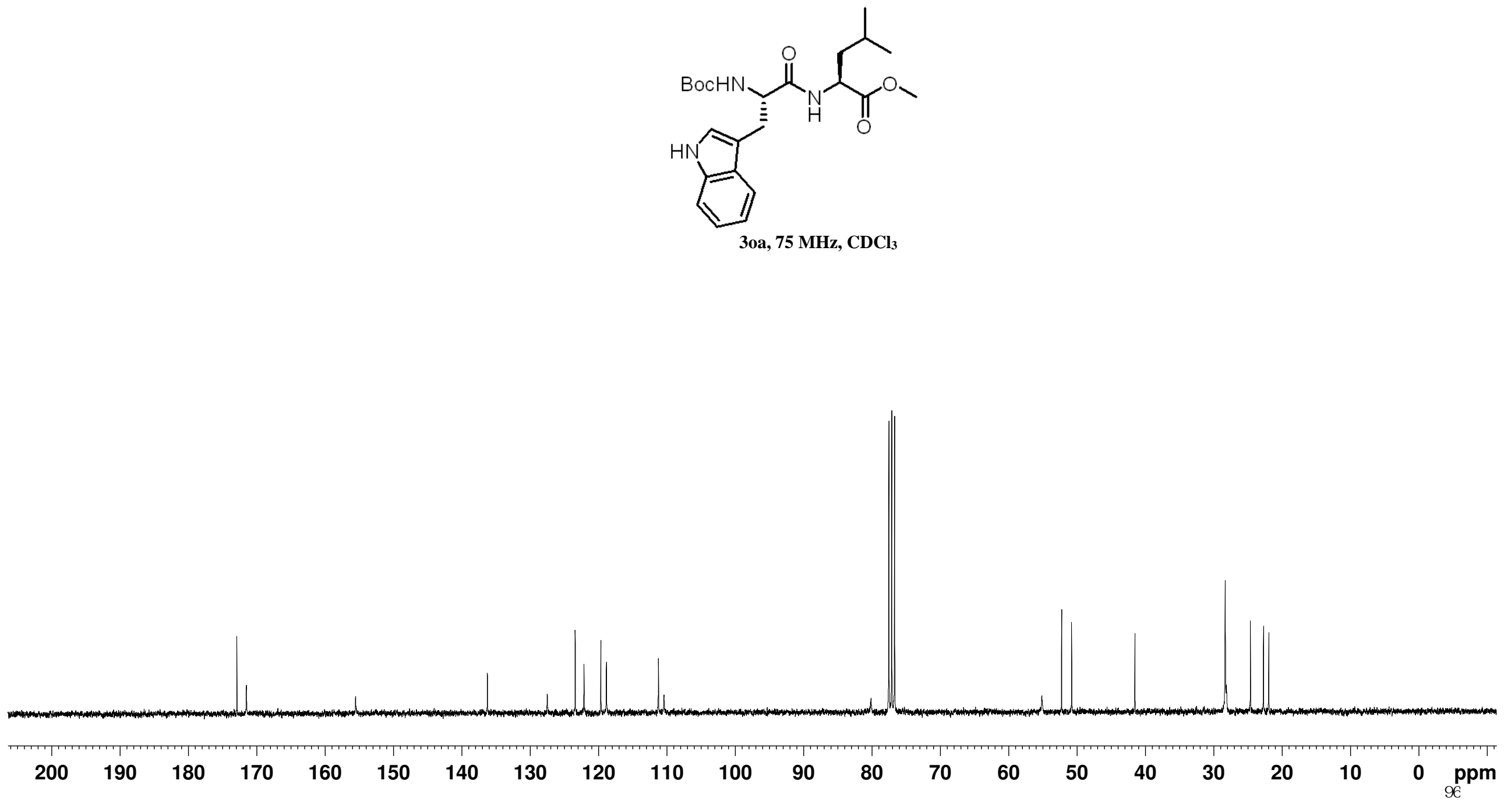


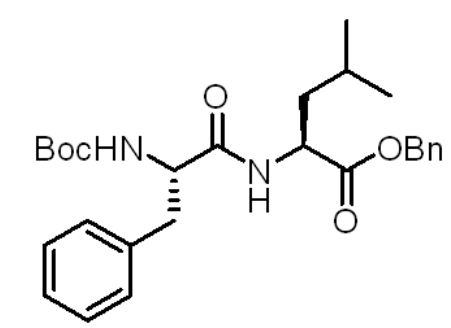

3pa, $300 \mathrm{MHz}, \mathrm{CDCl}_{3}$

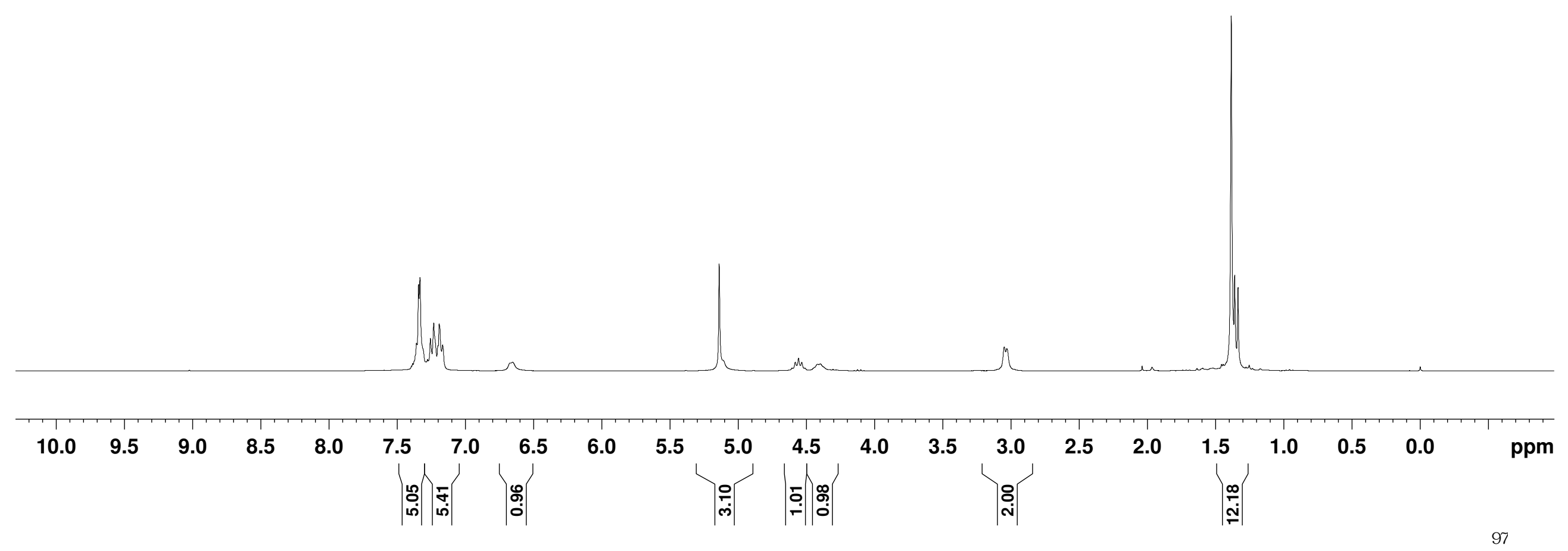




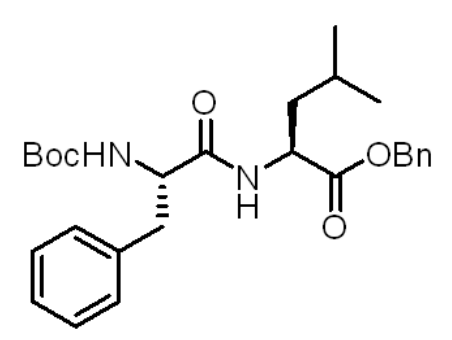

3pa, $75 \mathrm{MHz}, \mathrm{CDCl}_{3}$

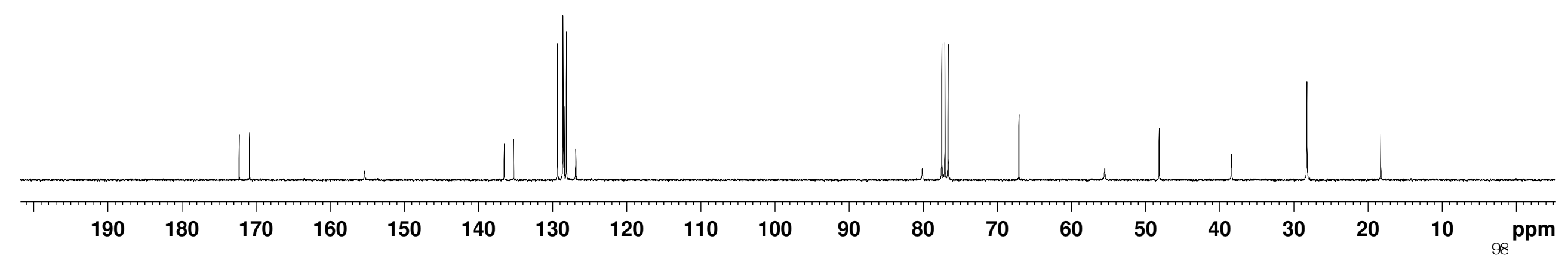




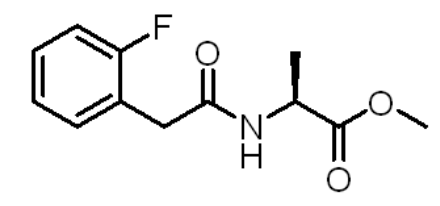

4a, $300 \mathrm{MHz}, \mathrm{CDCl}_{3}$

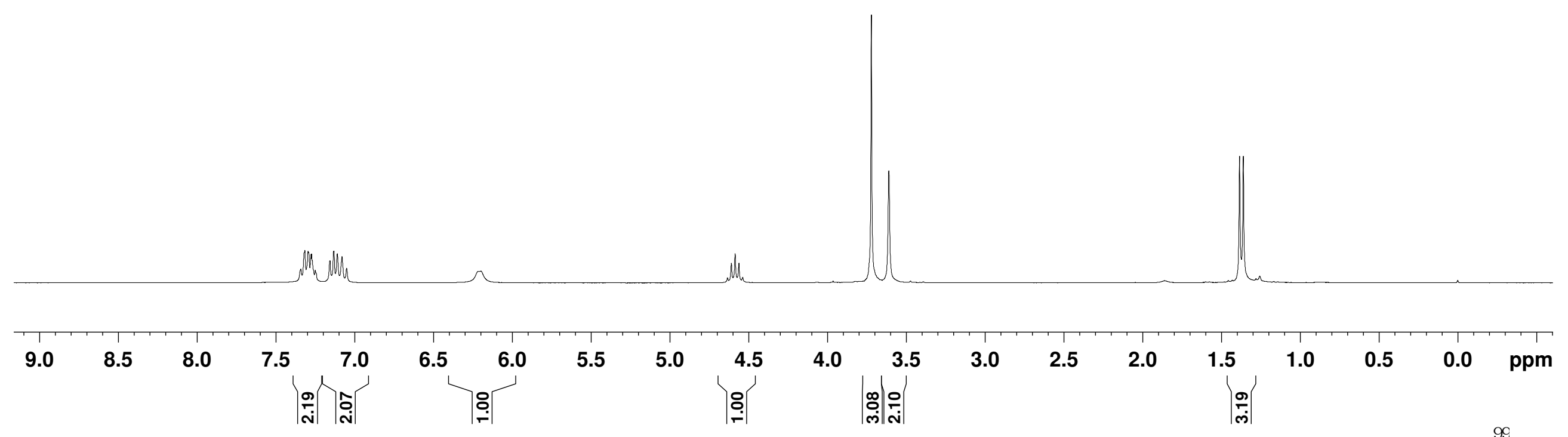




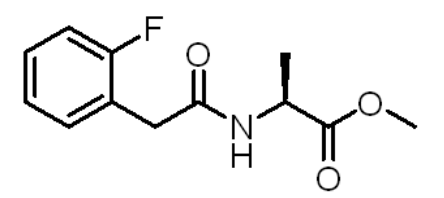

4a, $75 \mathrm{MHz}, \mathrm{CDCl}_{3}$

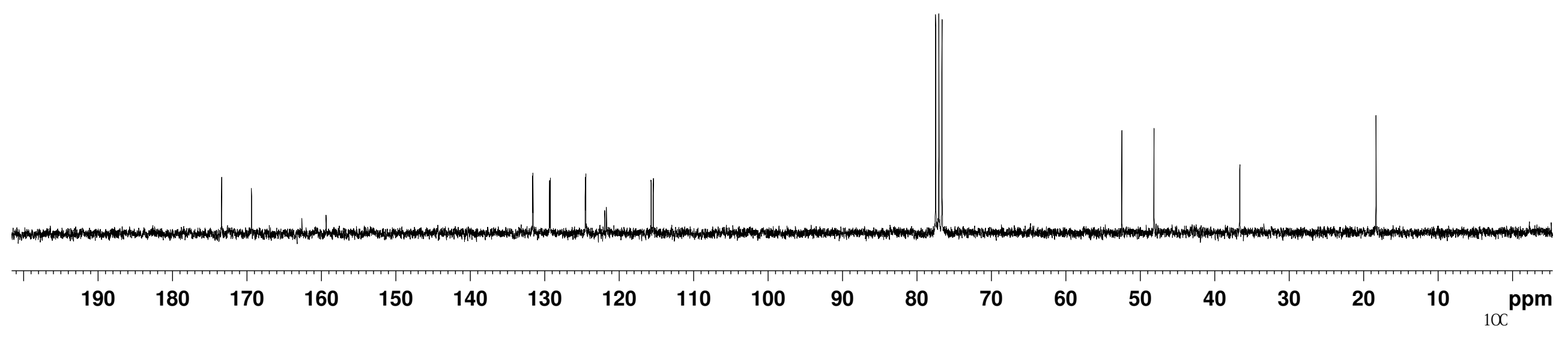




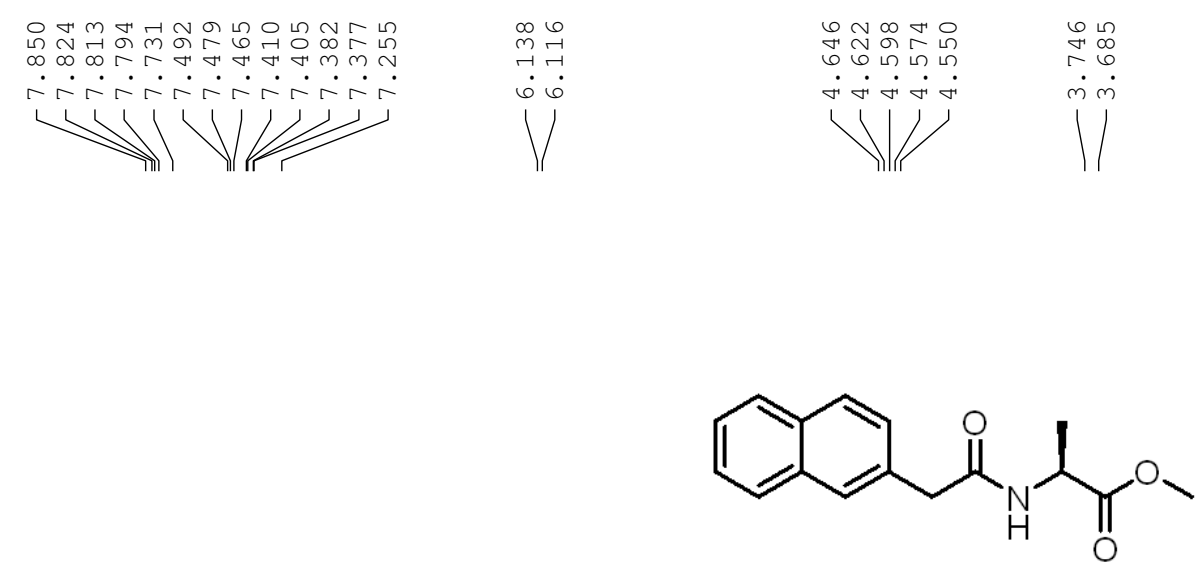

4b, $300 \mathrm{MHz}, \mathrm{CDCl}_{3}$

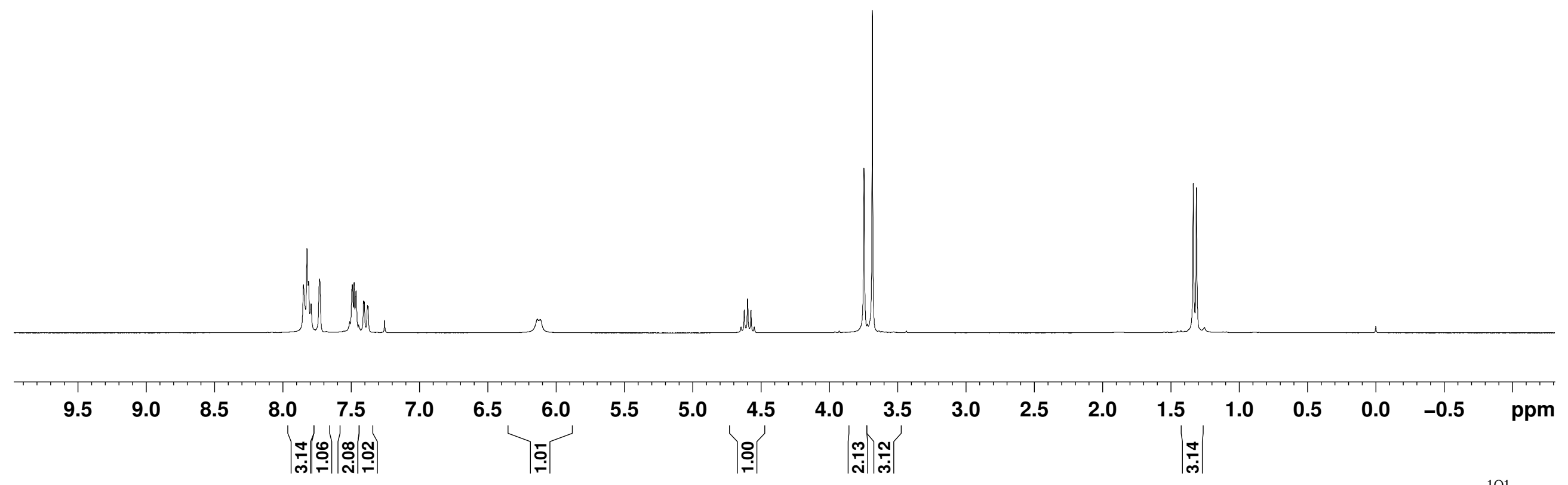




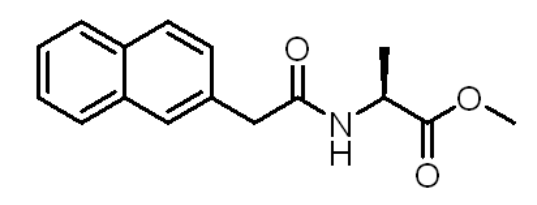

4b, $75 \mathrm{MHz}, \mathrm{CDCl}_{3}$

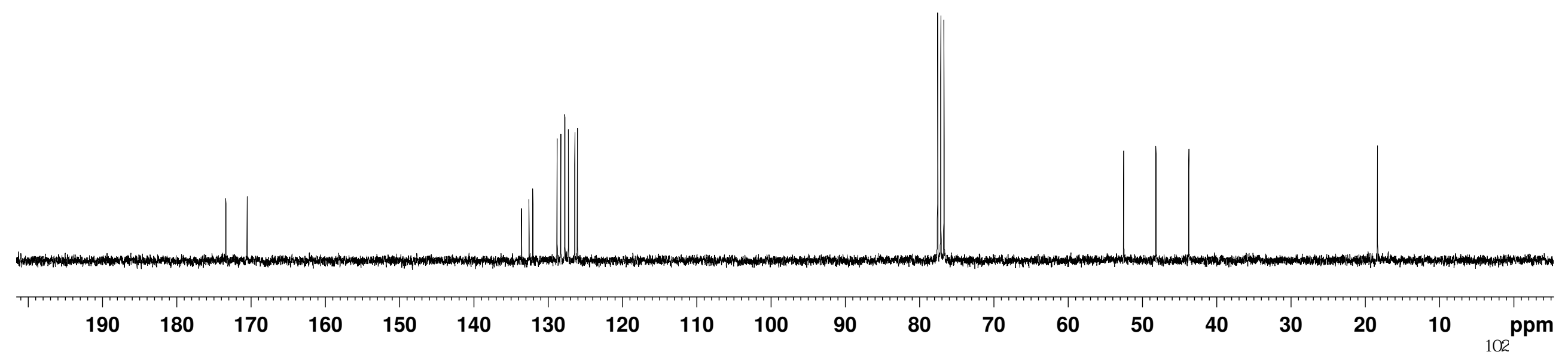



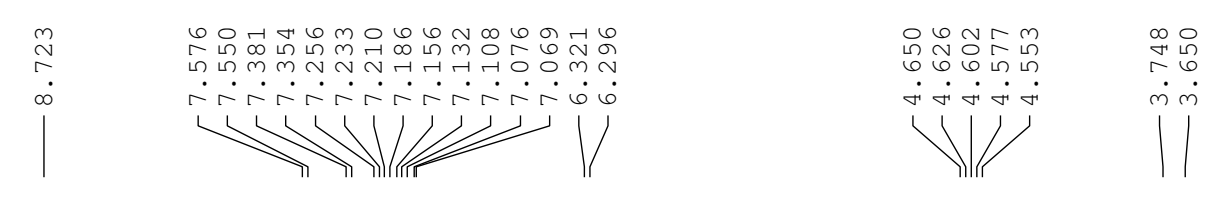

照

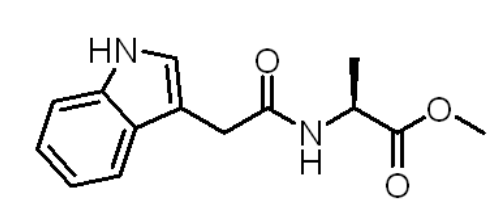

4c, $300 \mathrm{MHz}, \mathrm{CDCl}_{3}$

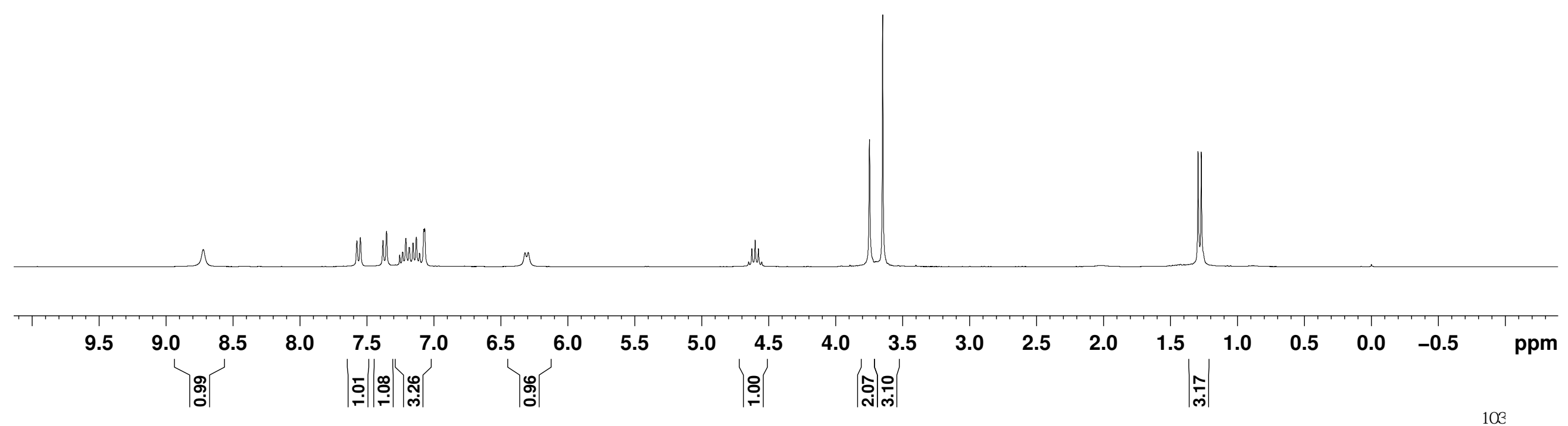




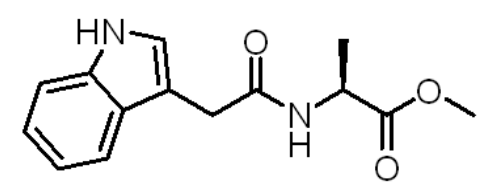

4c, $75 \mathrm{MHz} \mathrm{CDCl}_{3}$

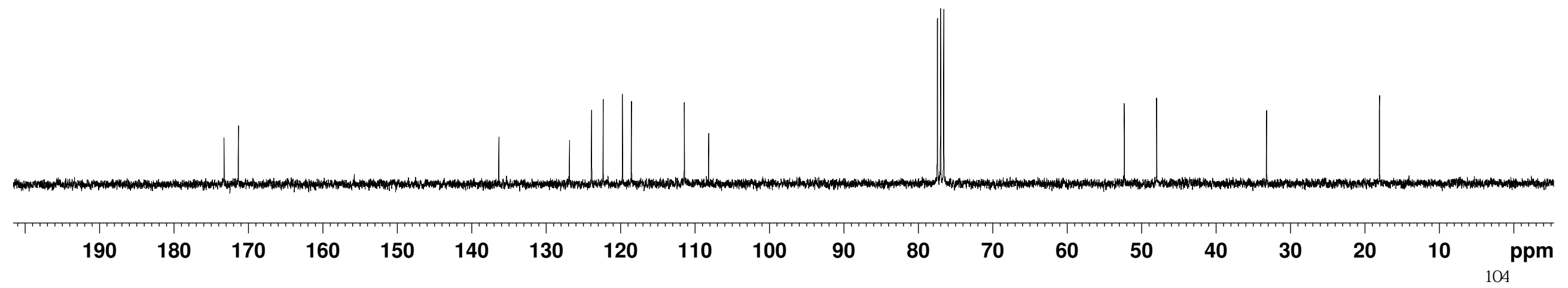




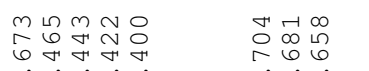

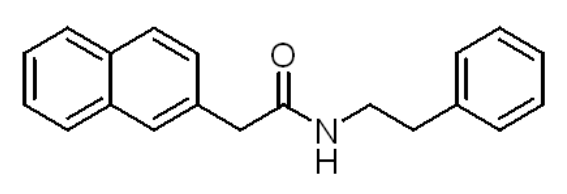

4d, $300 \mathrm{MHz}, \mathrm{CDCl}_{3}$

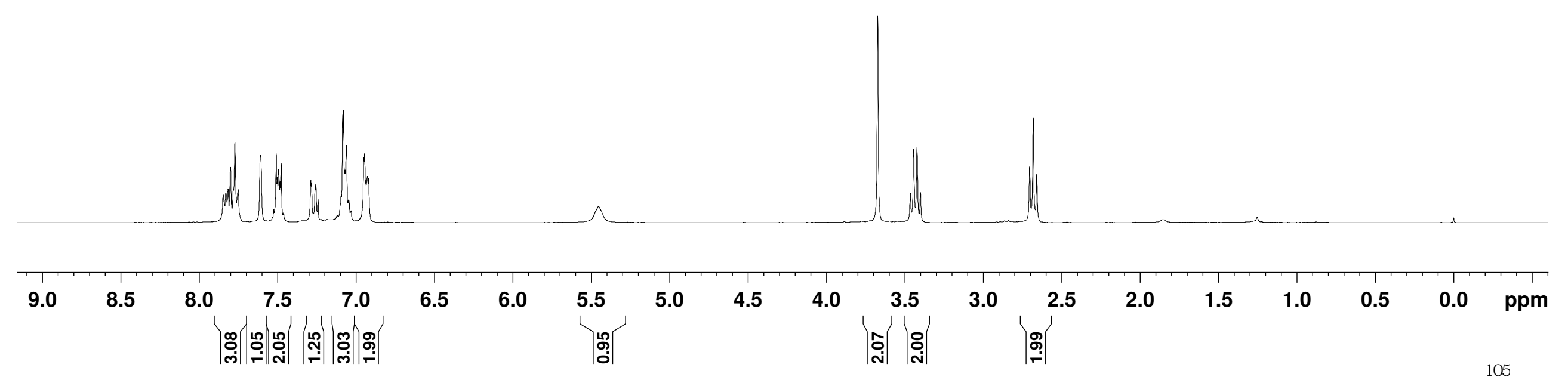




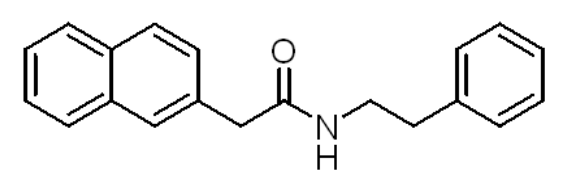

4d, $75 \mathrm{MHz}, \mathrm{CDCl}_{3}$

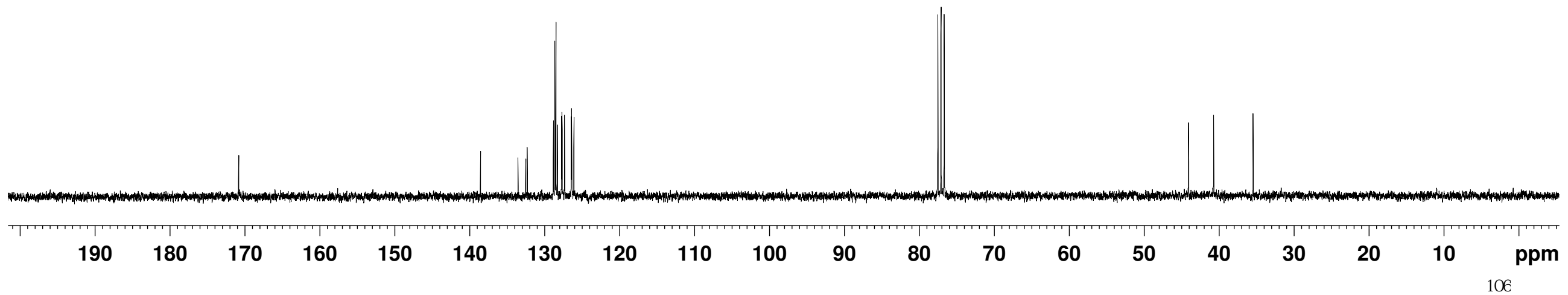




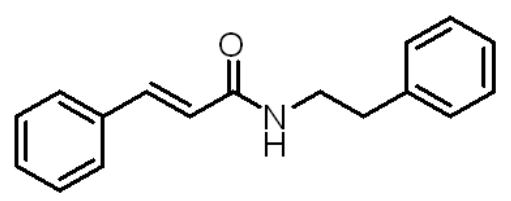

$4 \mathrm{e}, 300 \mathrm{MHz}, \mathrm{CDCl}_{3}$

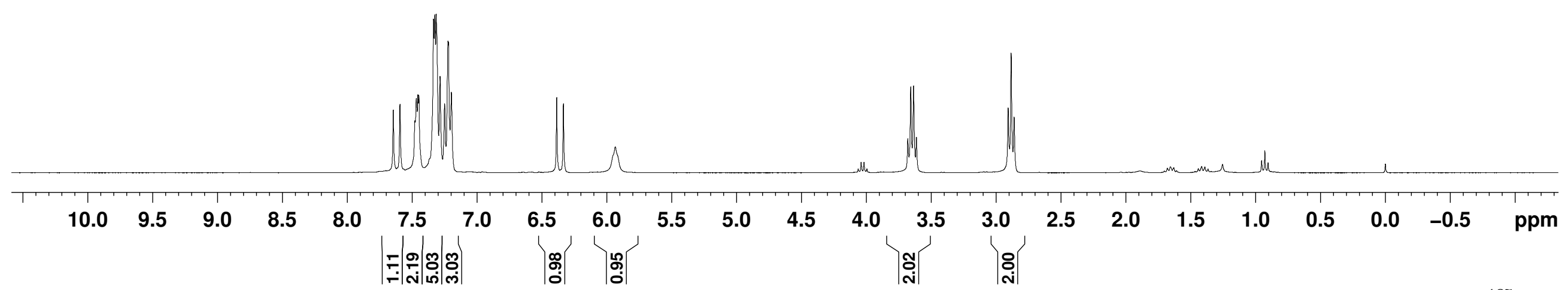




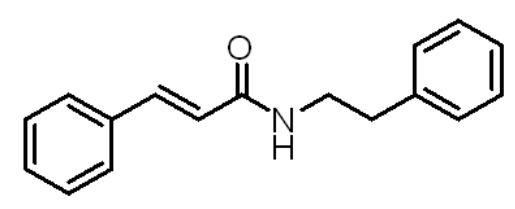

4e, 75 MHz, $\mathrm{CDCl}_{3}$

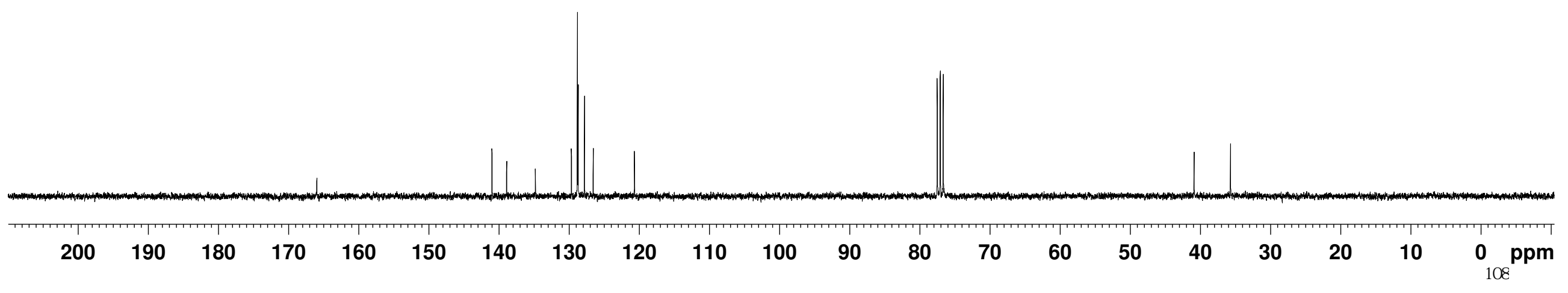



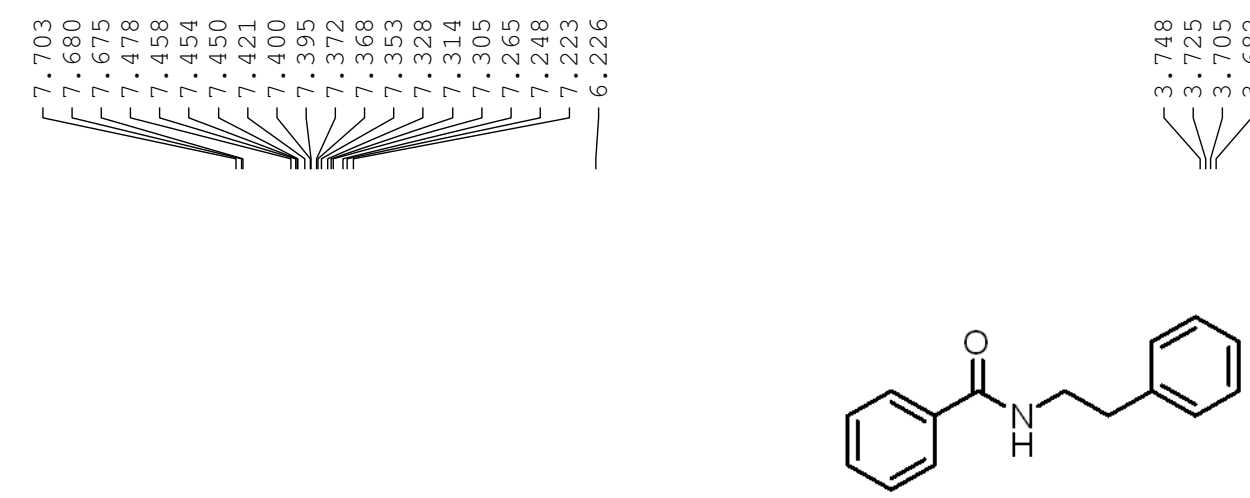

4f, $300 \mathrm{MHz}, \mathrm{CDCl}_{3}$

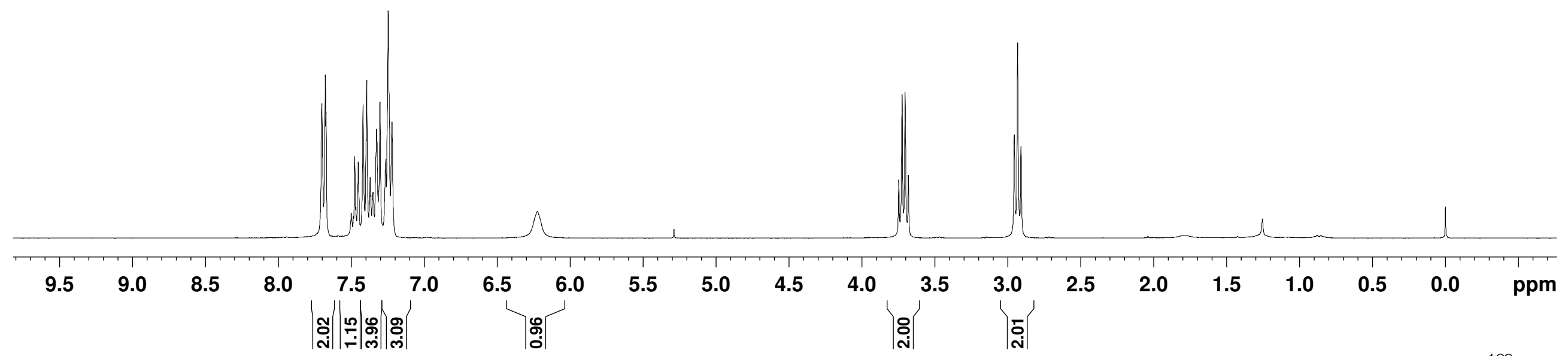



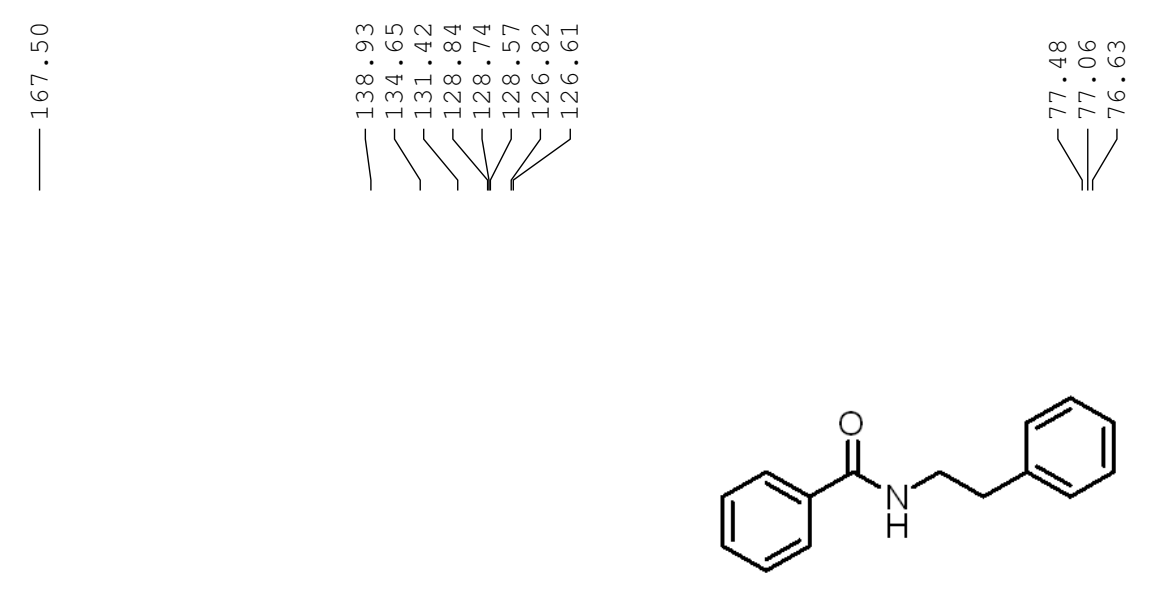

4f, $75 \mathrm{MHz}, \mathrm{CDCl}_{3}$

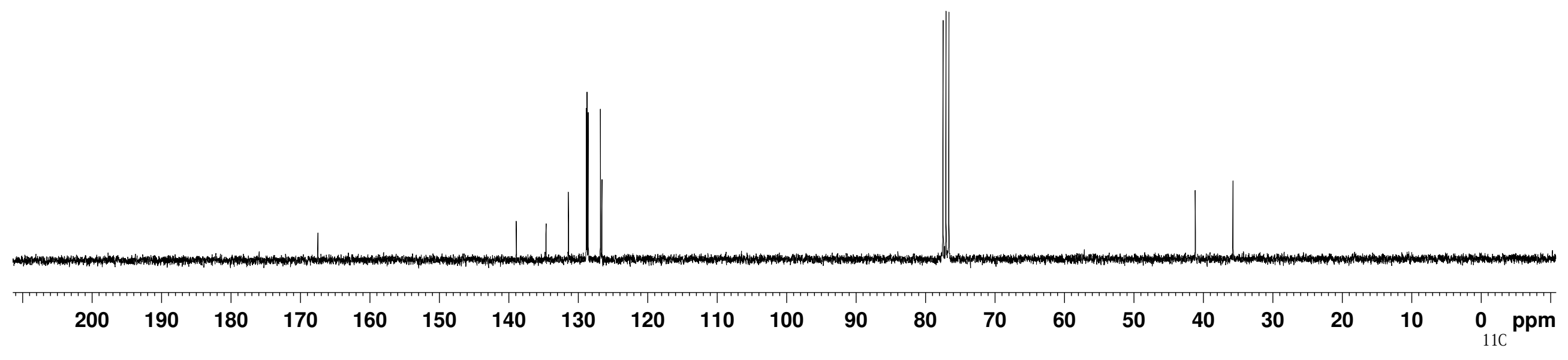



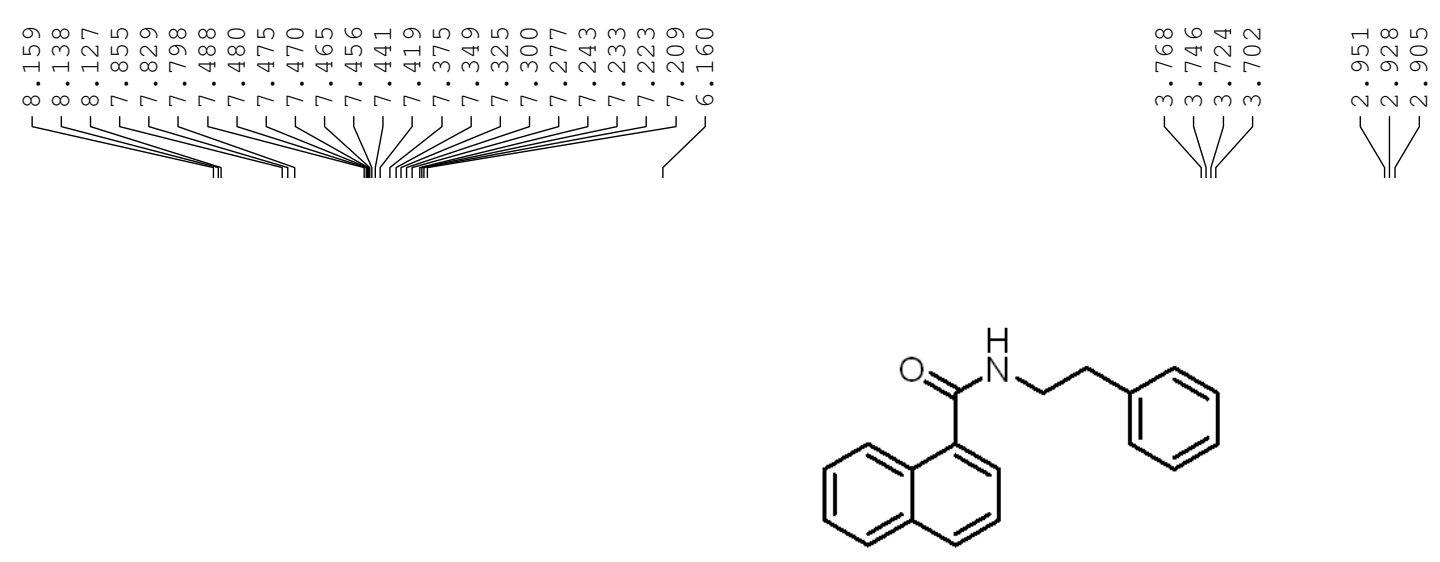

4g, $300 \mathrm{MHz}, \mathrm{CDCl}_{3}$

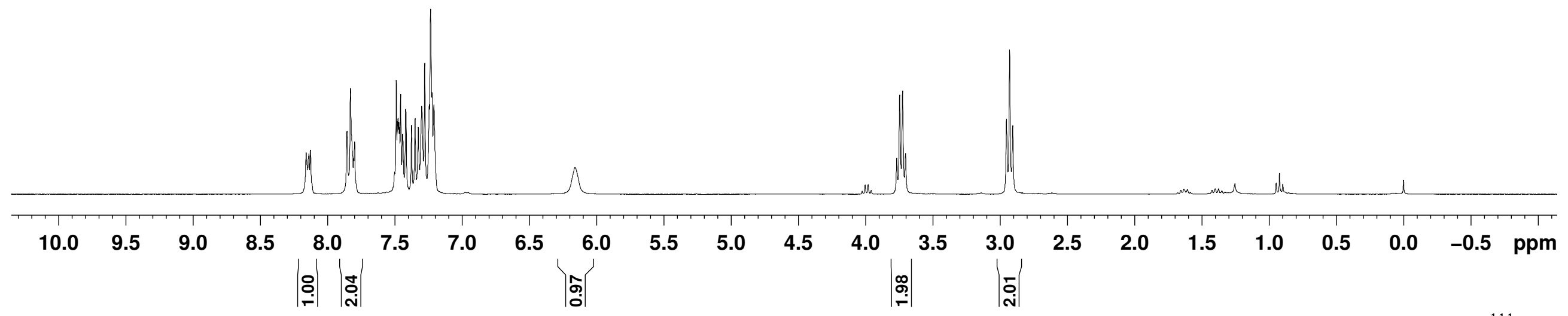



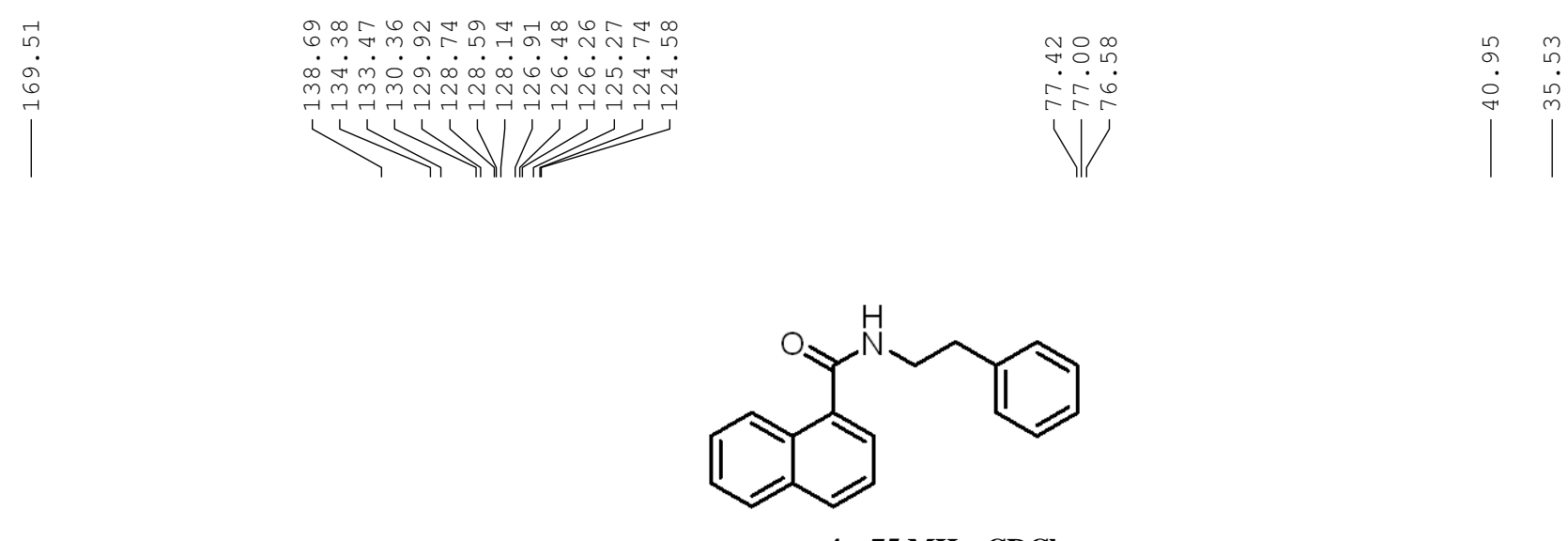

$4 \mathrm{~g}, 75 \mathrm{MHz}, \mathrm{CDCl}_{3}$

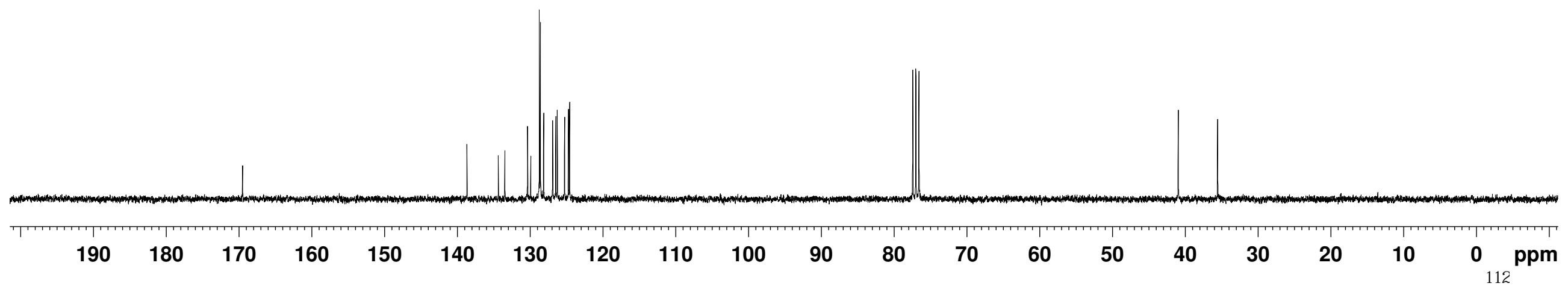




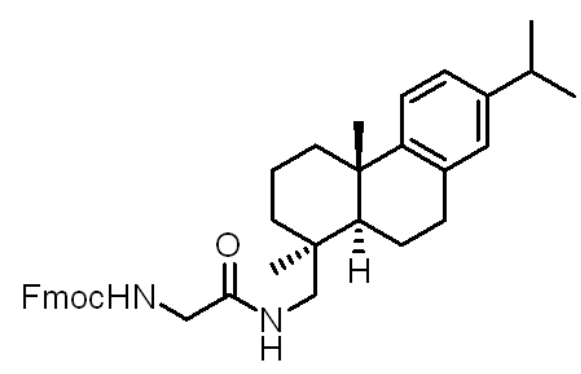

4h, $300 \mathrm{MHz}, \mathrm{CDCl}_{3}$

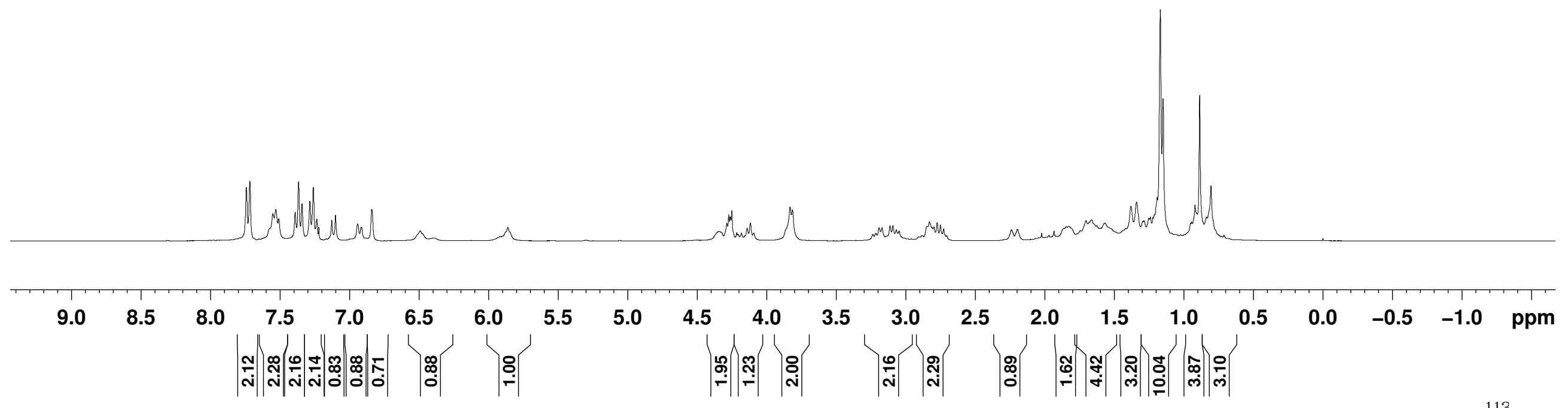




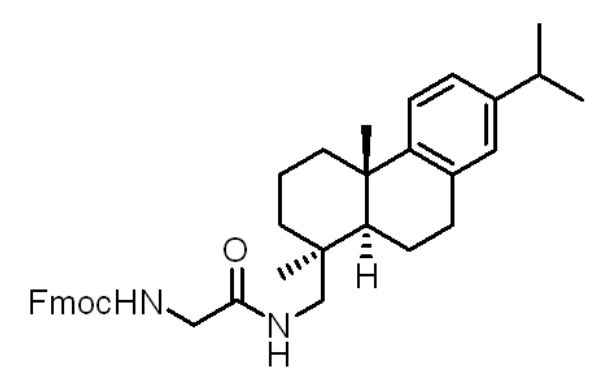

4h, $75 \mathrm{MHz}^{\mathrm{CDCl}_{3}}$

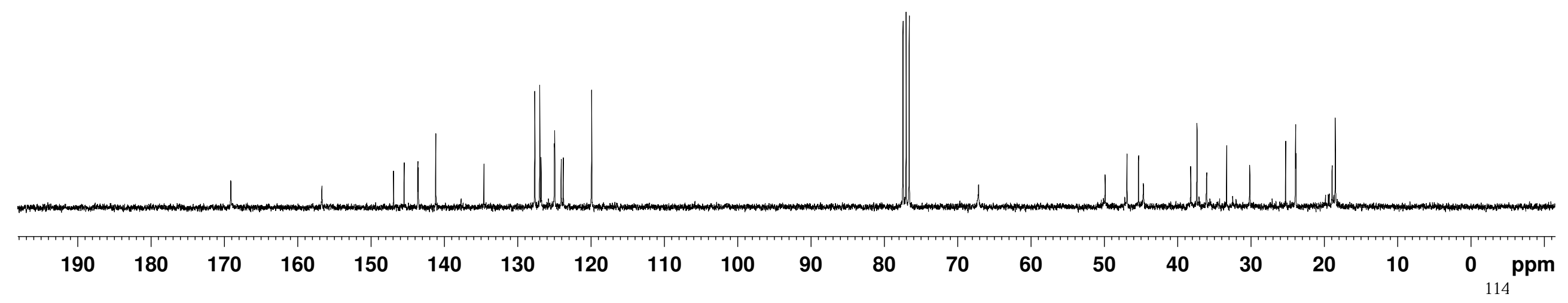




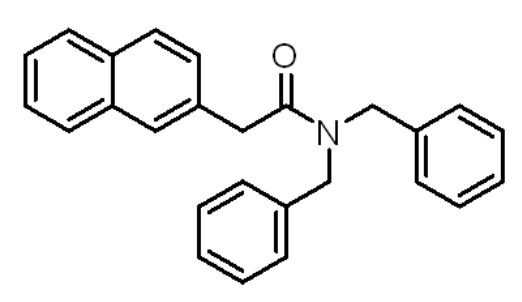

4i, $300 \mathrm{MHz}, \mathrm{CDCl}_{3}$

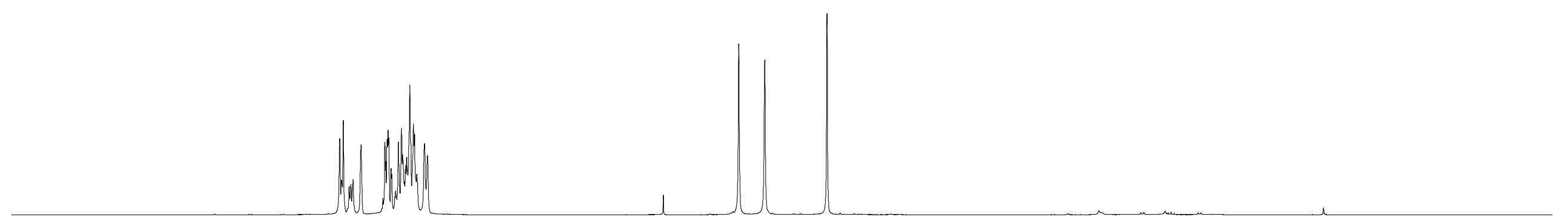

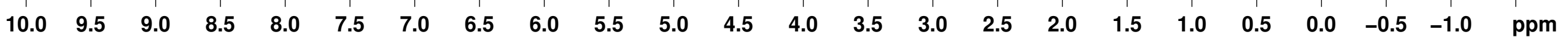

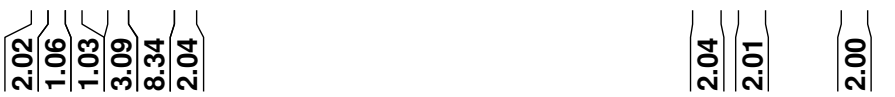




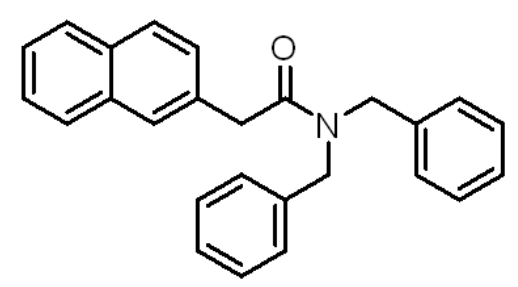

4i, $75 \mathrm{MHz}, \mathrm{CDCl}_{3}$

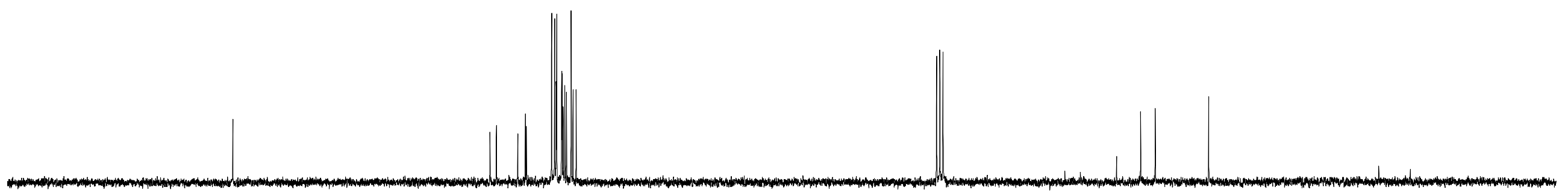


4j, $300 \mathrm{MHz}, \mathrm{CDCl}_{3}$

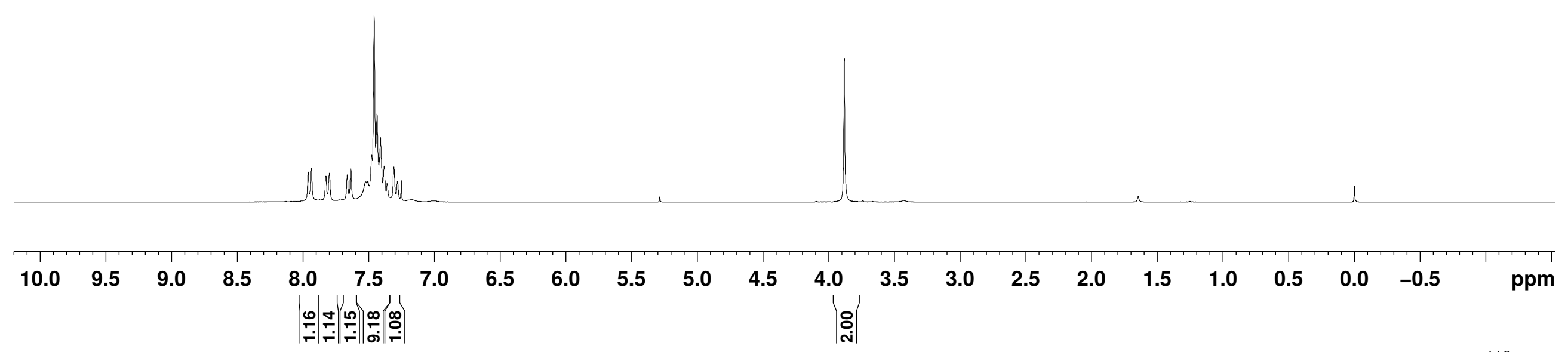




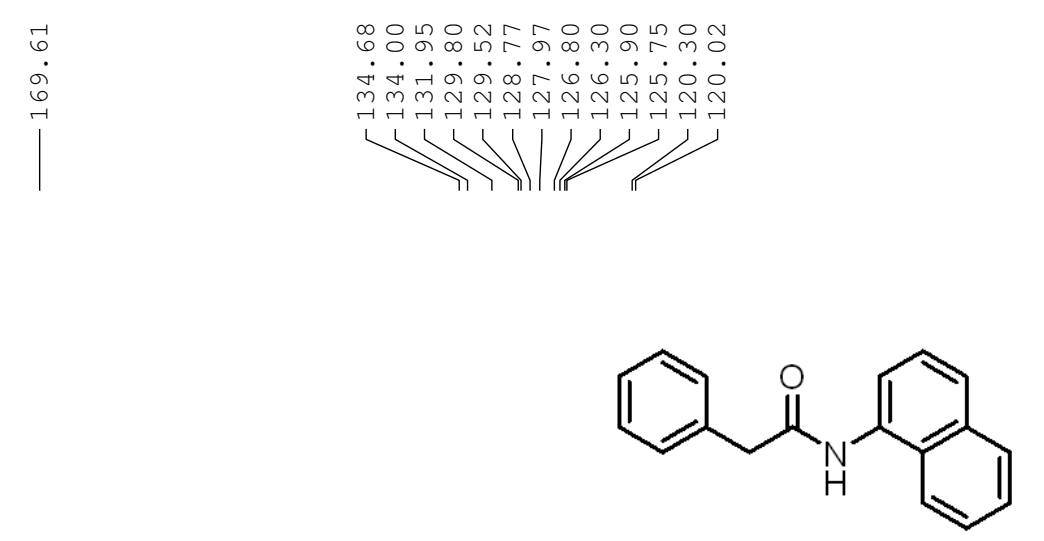

4j, $75 \mathrm{MHz}, \mathrm{CDCl}_{3}$

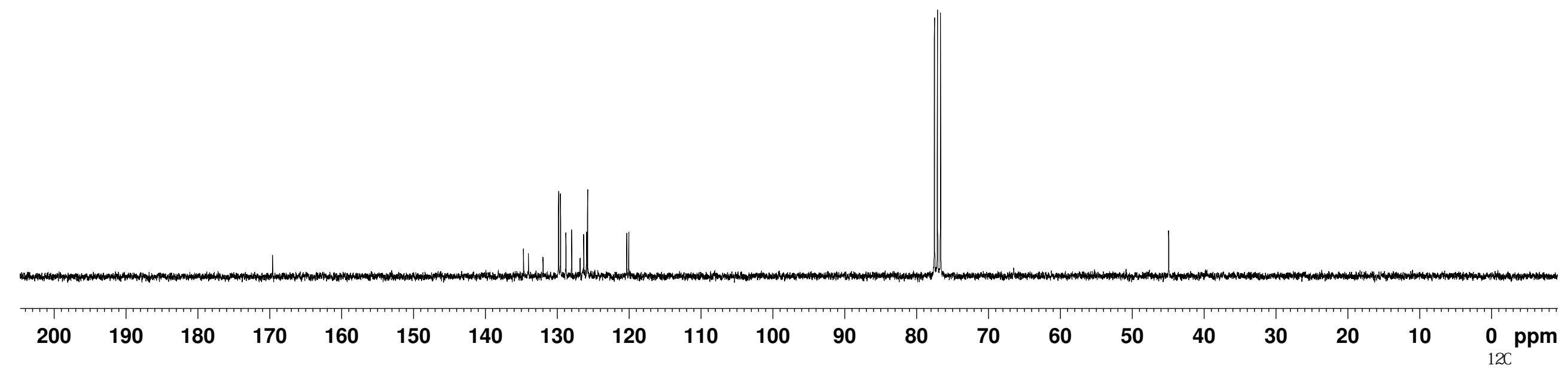




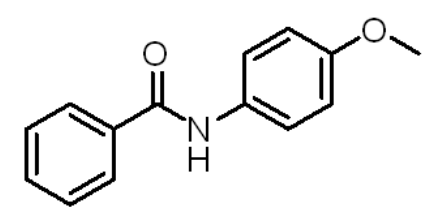

$4 \mathrm{k}, 300 \mathrm{MHz}, \mathrm{CDCl}_{3}$

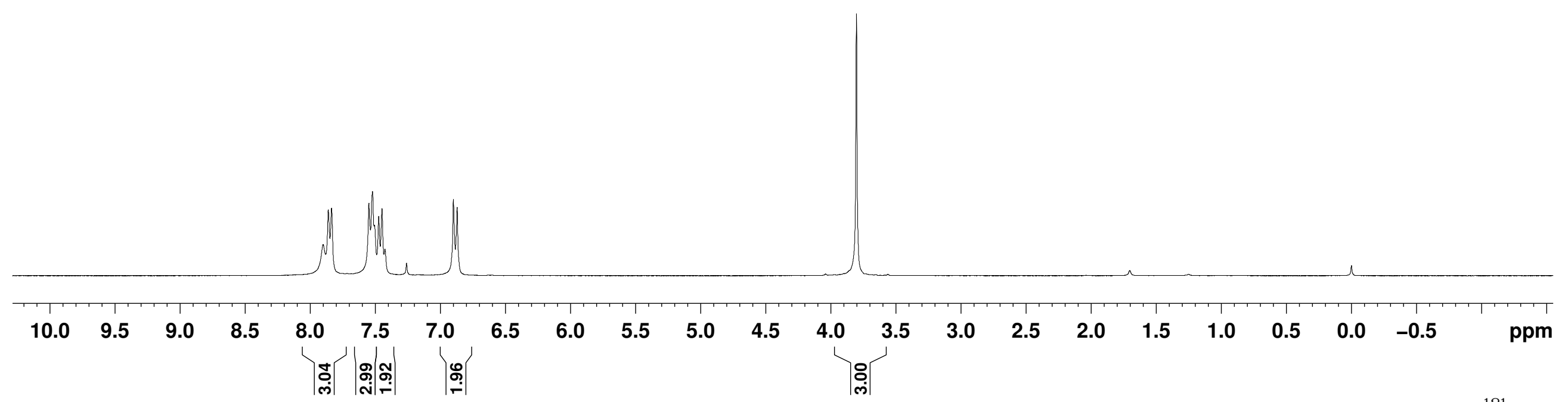




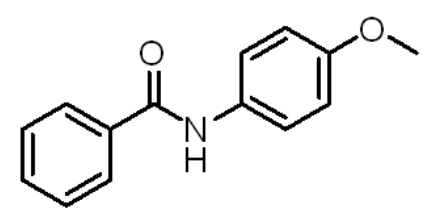

4k, $75 \mathrm{MHz}^{\mathrm{CDCl}_{3}}$

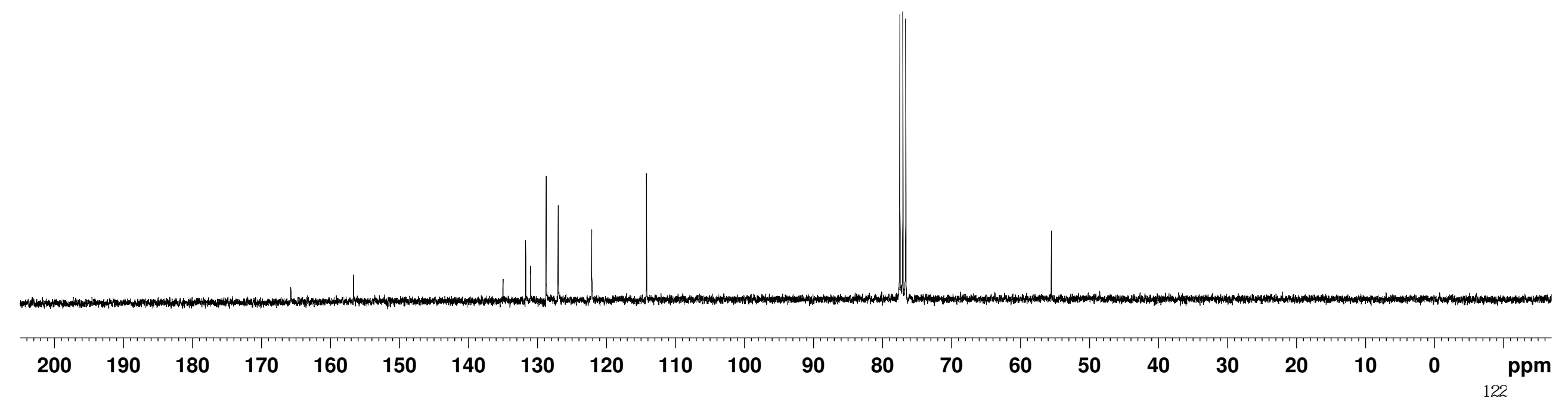




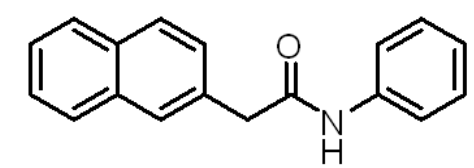

4l, $300 \mathrm{MHz}, \mathrm{CDCl}_{3}$

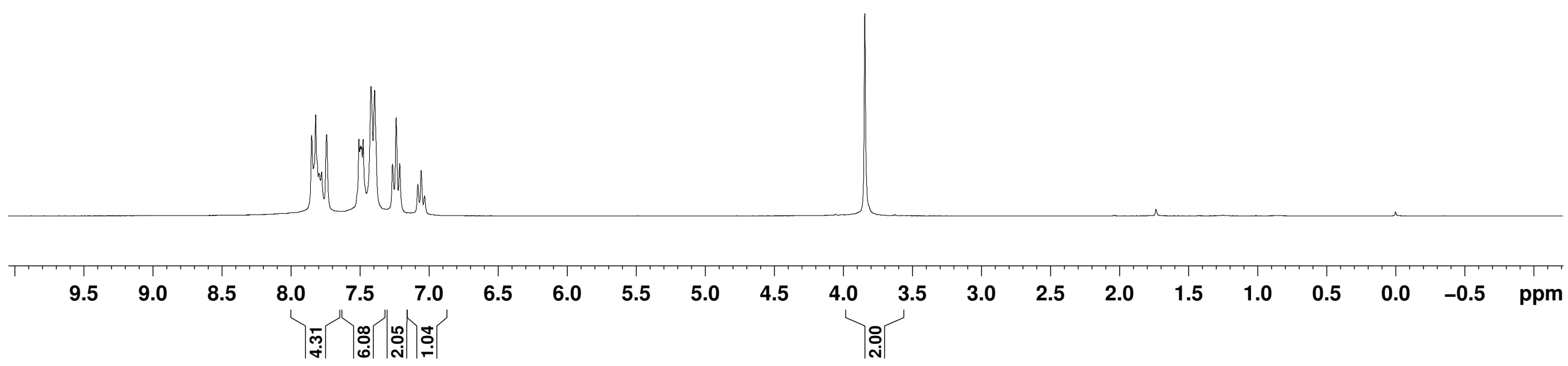




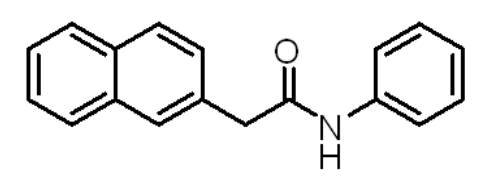

4l, $75 \mathrm{MHz}, \mathrm{CDCl}_{3}$

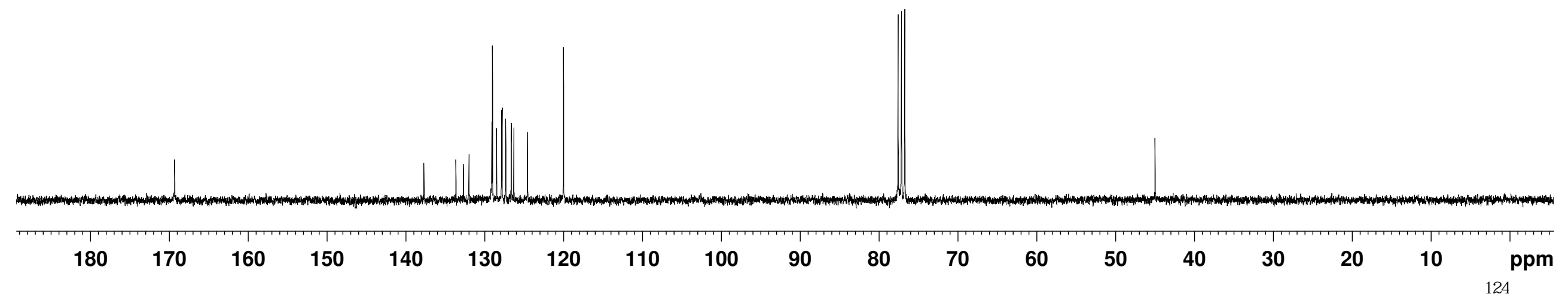




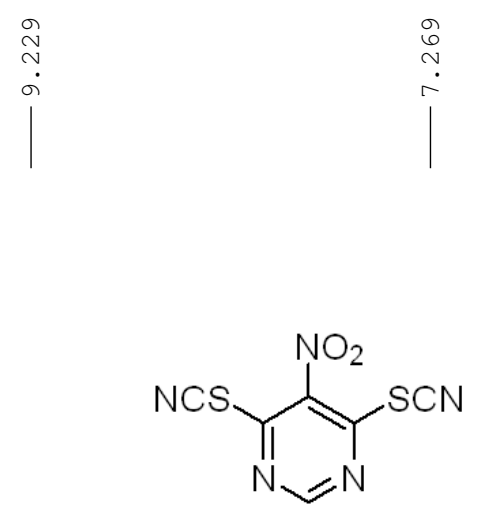

NDTP, $300 \mathrm{MHz}, \mathrm{CDCl}_{3}$

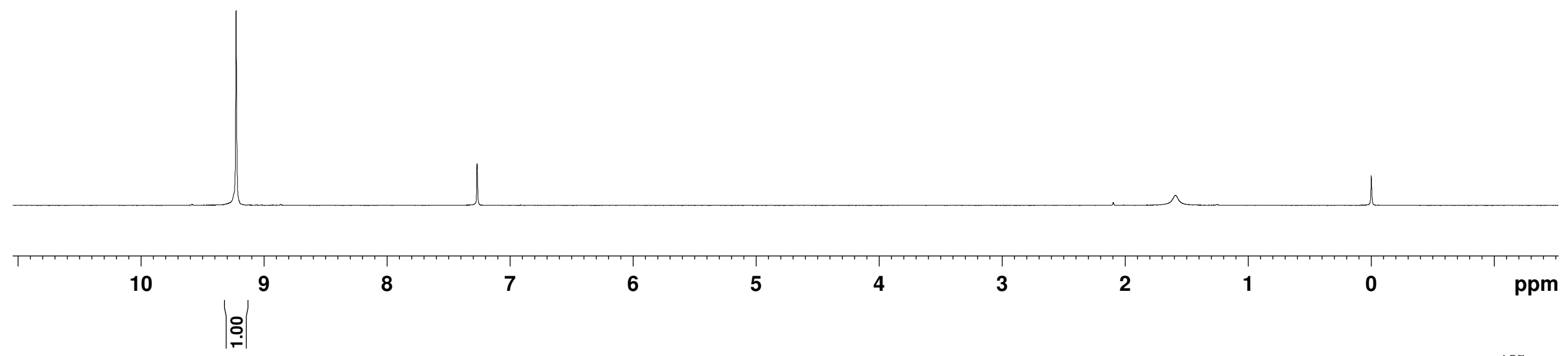


<smiles>N#Cc1ncnc([As])c1[N+](=O)[O-]</smiles>

NDTP, 75 MHz, $\mathrm{CDCl}_{3}$

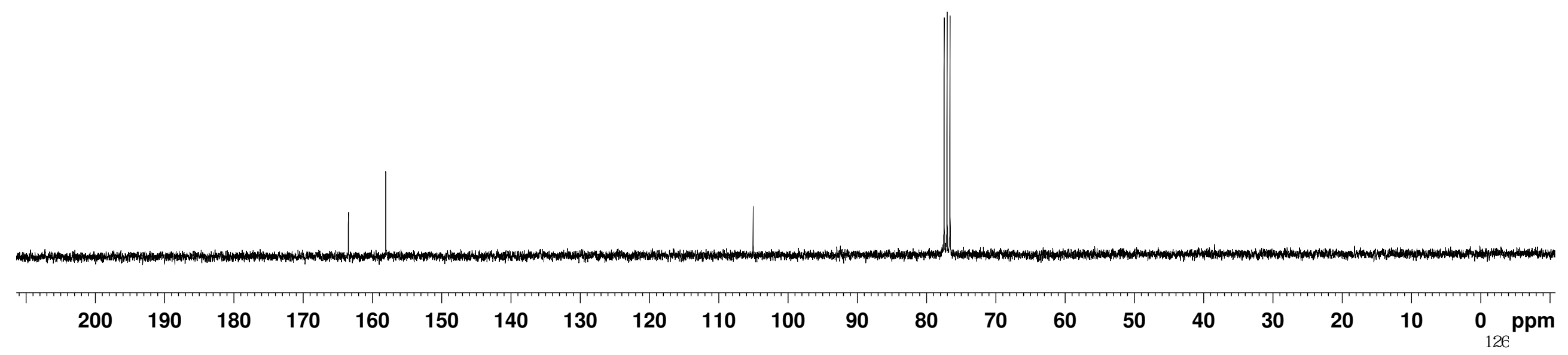




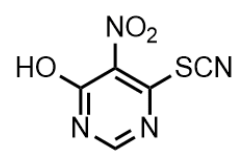

NTP-OH, 300 MHz, DMSO-d 6 


$$
\text { SCN }
$$

NTP-OH, 75 MHz, DMSO- $d_{6}$

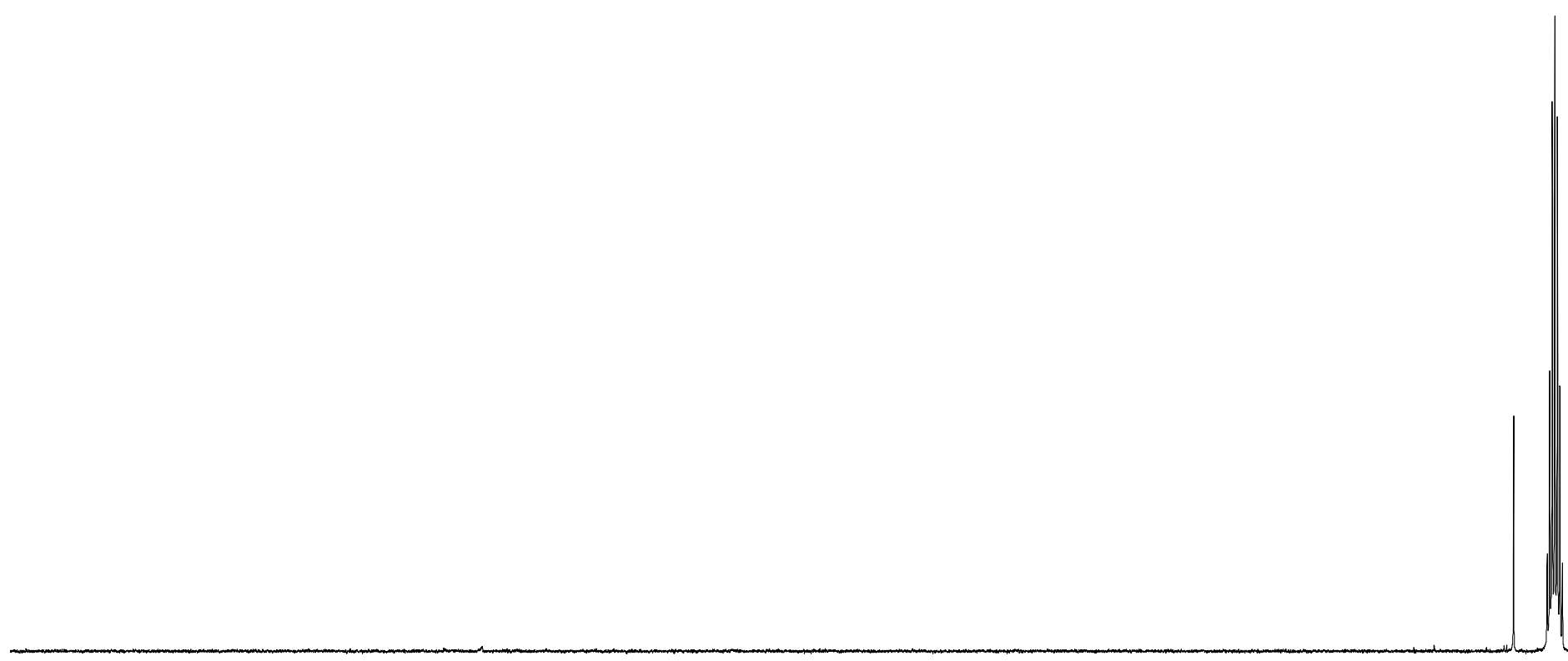

$\begin{array}{llllllllllllllllllllllll}200 & 190 & 180 & 170 & 160 & 150 & 140 & 130 & 120 & 110 & 100 & 90 & 80 & 70 & 60 & 50 & 40 & 30 & 20 & 10 & 0 & \text { ppm }\end{array}$



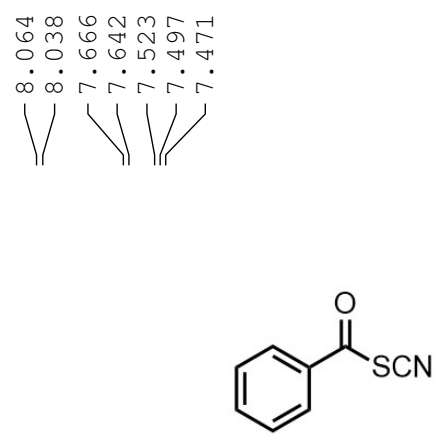

$300 \mathrm{MHz}, \mathrm{CDCl}_{3}$

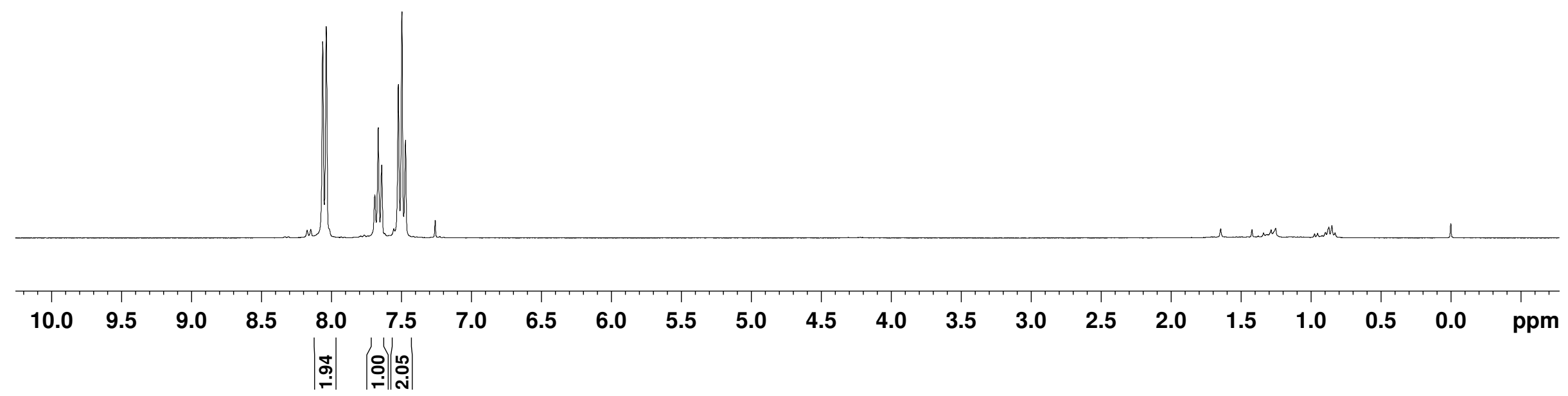



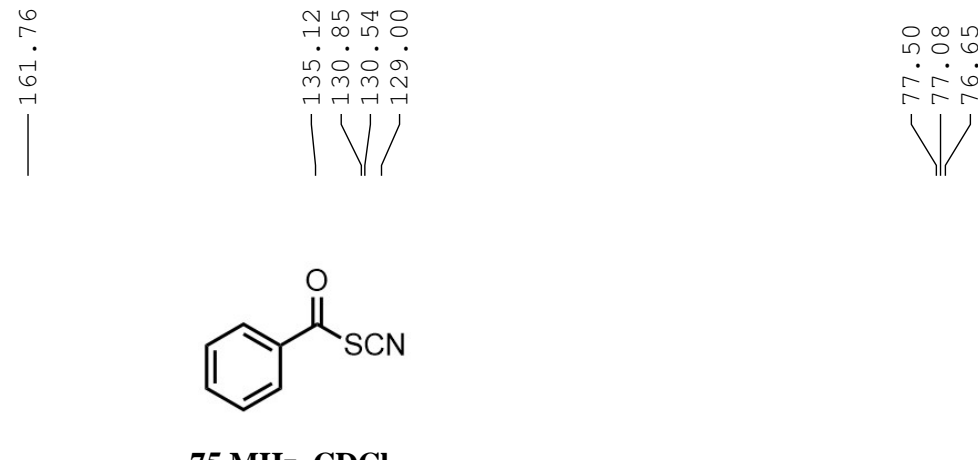

$75 \mathrm{MHz}, \mathrm{CDCl}_{3}$

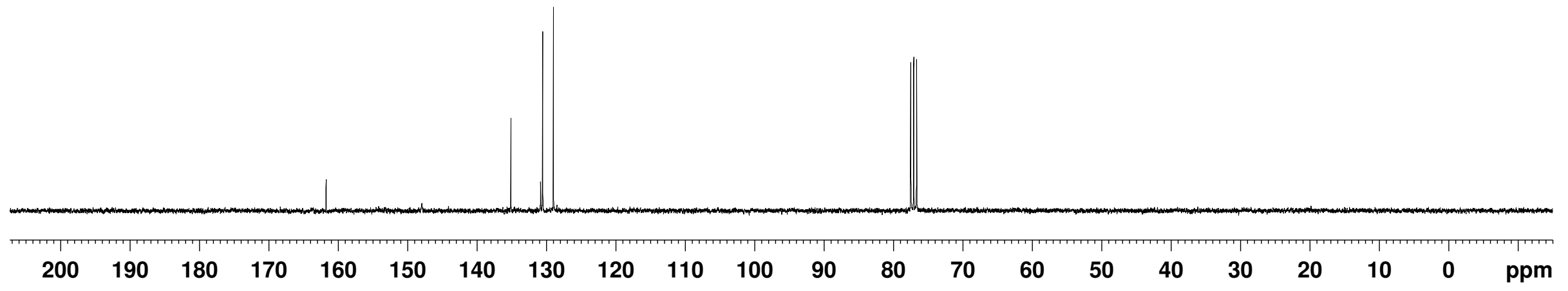

